\title{
Deformation of polyurea-coated steel plates under localised blast loading
}

\author{
by \\ Kathryn Patricia Ackland
}

Submitted in total fulfilment of the requirements of the degree of Doctor of Philosophy

January 2013

\section{Department of Infrastructure Engineering}

The University of Melbourne

Produced on archival quality paper 


\begin{abstract}
This thesis presents the results of experimental and numerical studies to investigate the effect of polyurea coatings on the deformation of steel plates under localised blast loading. Exploratory experiments and numerical modelling were initially performed to develop an explosive test methodology and provide validation data for preliminary numerical modelling. Using the developed methodology, explosive testing was conducted on bare and polyurea-coated steel plates, where the plate configurations all had the same areal density. It was found that the polyurea-coated plates resulted in higher residual deformations compared to the uncoated plates, with the residual deformations increasing with coating thickness (and hence increasing with thinner steel). High speed video footage of the events revealed that the polyurea coatings debonded and hyper-extended during the events, before coming to rest back against the plate. This resulted in transient deformations of the polyurea coatings which were approximately twice that of the bare steel plates.
\end{abstract}

Following the explosive experiments, numerical modelling of the polyurea-coated plates under blast loading was conducted using AUTODYN ${ }^{\circledR}$. The dimensions of the numerical mesh were selected through a sensitivity study. De-bonding of the polyurea was reproduced by using a thin layer of elements which failed at a designated principal stress value, which was tuned to fit the experimental measurements. The polyurea was modelled using a two-parameter Mooney-Rivlin relationship, the constants for which were validated in the preliminary simulations. The initial models of the explosive experiments showed excellent agreement with the experimental residual deformations of the plates, but under-predicted the peak transient deformations of the polyurea. To improve the model accuracy, tensile tests were conducted on the polyurea at various strain rates and used to fit new Mooney-Rivlin material model constants. The new material model constants gave improved results for the transient deformations of the polyurea coatings.

Parametric studies were conducted using the validated material models to investigate the effect of bond strength, polyurea stiffness, polyurea bulk modulus and the coating location. It was found that the greatest improvement could be achieved by changing 
the coating location to the front (blast side) face of the steel plate instead of the back face. The coating performance could also be improved by increasing the polyurea stiffness or bulk modulus, or increasing the strength of the bond between the polyurea and the plate. None of the polyurea-coated plates in the parametric studies performed as well as a mass-equivalent steel plate. However, polyurea-coated steel plates may still be preferable for other reasons, for example when the coating is to be applied as an appliqué (add-on) armour. 


\section{Declaration}

This is to certify that:

i. the thesis comprises only my original work towards the PhD,

ii. due acknowledgement has been made in the text to all other material used,

iii. the thesis is less than 100,000 words in length, exclusive of tables, maps, bibliographies and appendices.

Kathryn Patricia Ackland

July 2013 


\section{Acknowledgements}

I would like to thank my supervisors, Prof. Priyan Mendis and Dr. Tuan Ngo from

The University of Melbourne, and Dr. Chris Anderson from the Defence Science and Technology Organisation, for their guidance and support throughout my PhD candidature. I would also like to extend my gratitude to Dr. Shannon Ryan, for his assistance and patience in helping me finish this thesis during my final year of candidature.

Many thanks to the Maritime Platforms Division and DSTO senior management who have allowed me the opportunity to complete this $\mathrm{PhD}$. Thankyou also to the $\mathrm{DSTO}$ National Security and Technology Centre for providing funding and support which allowed me to complete this work.

I would like to extend a big thanks to my colleagues and friends from Maritime Platforms Division at DSTO. From the trials team to proof-readers and many others: your support, advice and encouragement have made the work in this thesis possible.

I would also like to thank the staff and fellow students from the University of Melbourne's Department of Infrastructure Engineering who have worked alongside me over the years.

Finally, I would like to thank my family and friends for their love and understanding throughout my PhD studies. I would especially like to thank my parents, Sue and Bruce, and my fiancé Paul, with whom I look forward to sharing a very special bottle of champagne when my $\mathrm{PhD}$ journey is complete. 


\section{Table of Contents}

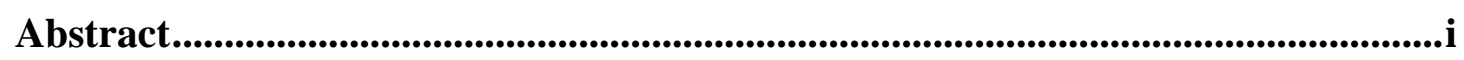

Declaration.......................................................................................................... iii

Acknowledgements .........................................................................................................iv

Table of Contents .......................................................................................................

List of Figures...........................................................................................................................ix

List of Tables .................................................................................................... Xviii

List of Symbols and Abbreviations ..........................................................................xix

Chapter 1 Introduction.........................................................................................1

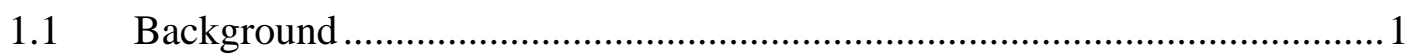

1.2 Problem description and research questions .................................................

1.3 Research framework and scope .................................................................

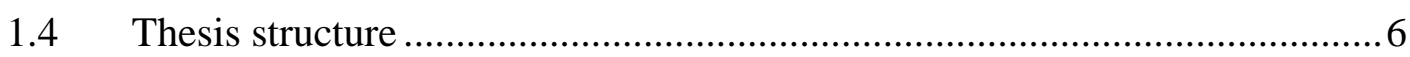

Chapter 2 Literature Review ....................................................................................8

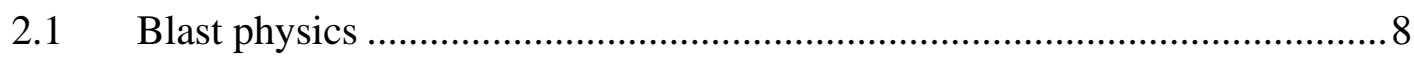

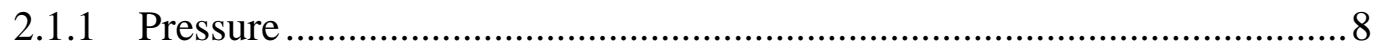

2.1.2 Blast wave formation ............................................................................. 10

2.1.3 Blast effects on a structure.....................................................................12

$2.2 \quad$ Plate response to blast loads .......................................................................

2.2.1 Key mechanisms of blast protection ....................................................... 14

2.2.2 Polyurea coatings for blast mitigation ...................................................... 17

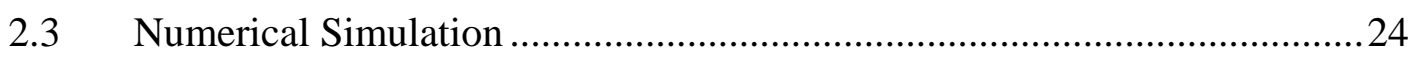

2.3.1 Theoretical studies .................................................................................2 25

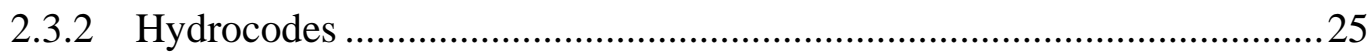

2.3.3 Simulation of blast/structure interaction ..................................................26 
2.3.4 Using finite elements to model plates ...............................................27

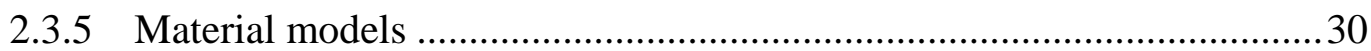

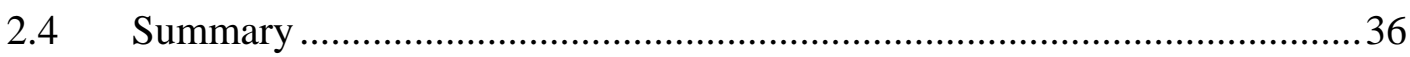

Chapter 3 Preliminary Experiments and Numerical Validation ............................37

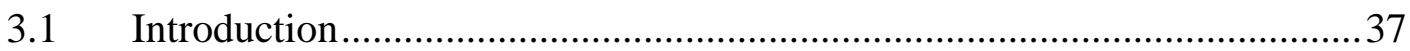

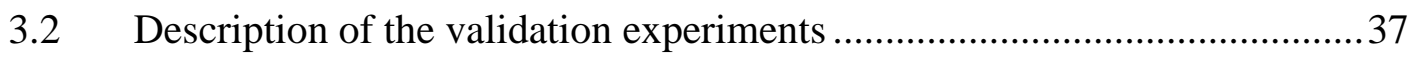

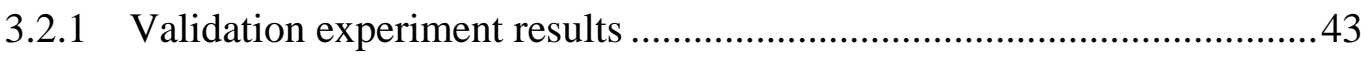

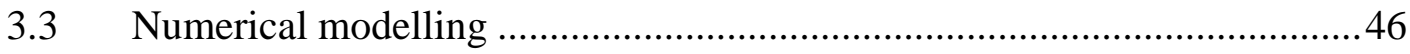

3.3.1 Numerical modelling approach............................................................46

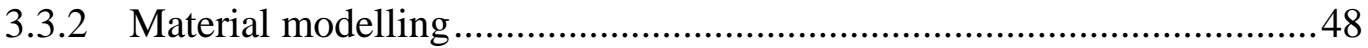

3.3.3 Mesh discretisation of the steel plate .......................................................50

3.3.4 Mesh discretisation of the polyurea coating ..........................................58

3.3.5 Mesh discretisation of Euler grid ........................................................6 60

3.4 Numerical simulation and comparison with the experimental results: bare

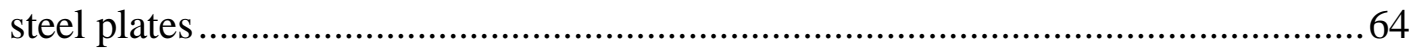

3.5 Numerical simulation and comparison with the experimental results:

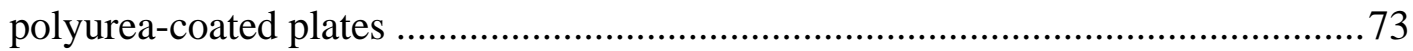

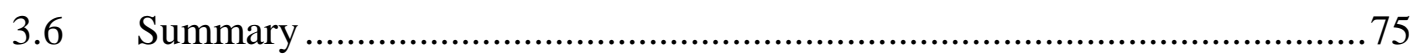

Chapter 4 Explosive Testing of Polyurea-Coated Steel Plates...............................77

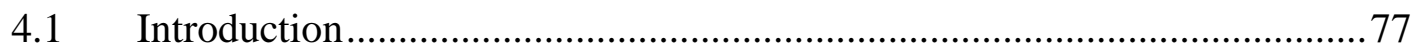

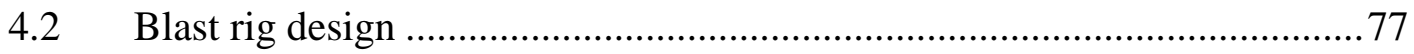

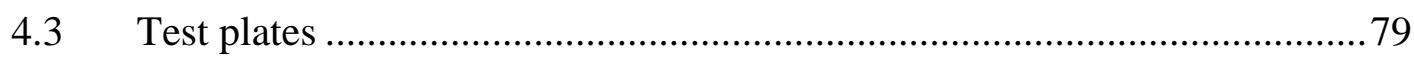

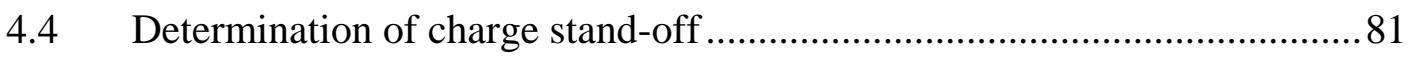

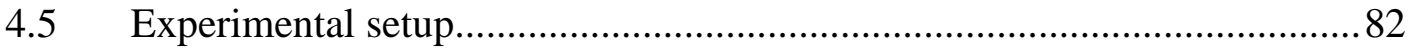

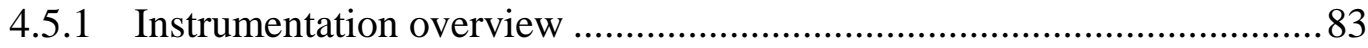

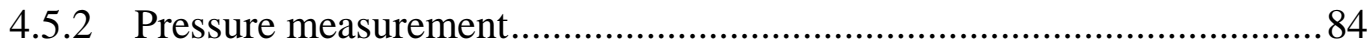

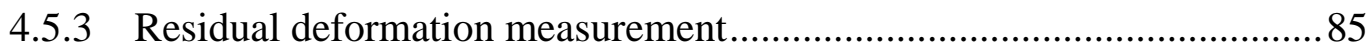

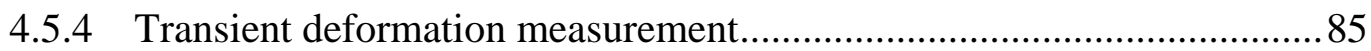

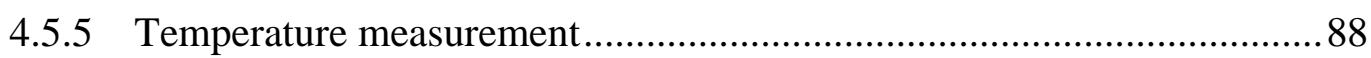

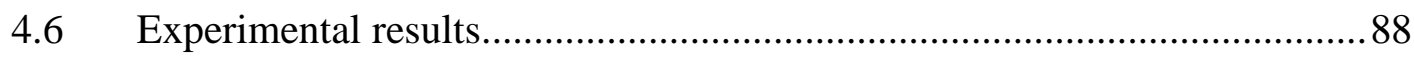

4.6.1 Validation of charge detonation ............................................................ 88 


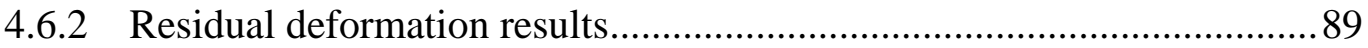

4.6.3 Effect of coating thickness on residual deformation...............................94

4.6.4 Effect of charge mass on residual deformation......................................95

4.6.5 Effect of temperature on residual deformation .....................................96

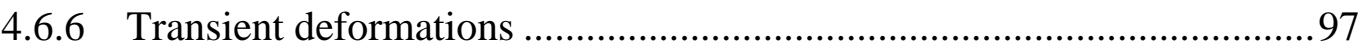

4.6.7 Plate boundary movement measurement .............................................104

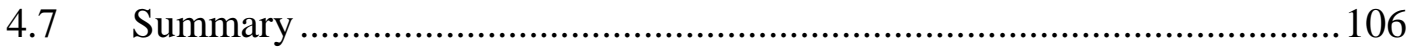

Chapter 5 Numerical Simulations and Parametric Studies .................................108

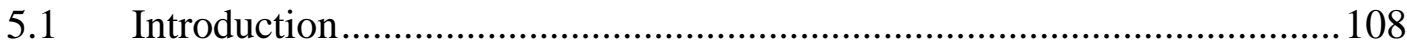

$5.2 \quad$ Numerical modelling of the explosive experiments .................................108

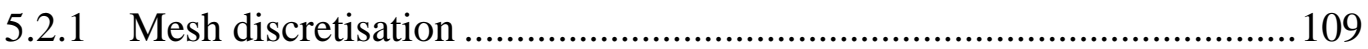

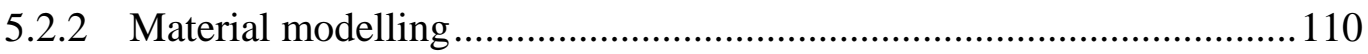

5.2.3 Modelling of polyurea de-bonding ...................................................113

5.2.4 Numerical modelling results and comparison with the experiments .....124

5.3 Refinement of the Mooney-Rivlin constants ...........................................132

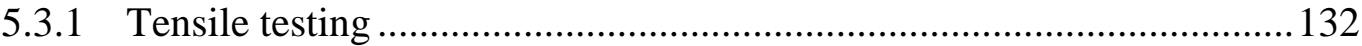

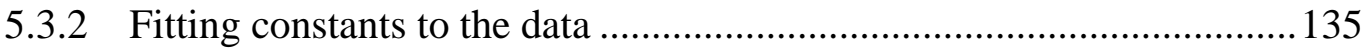

5.4 Numerical modelling of the experiments with new material model constants

$5.5 \quad$ Parametric modelling studies .................................................................. 143

5.5.1 Variations in bond strength ............................................................. 144

5.5.2 Variations in polyurea stiffness …...................................................147

5.5.3 Variations in coating bulk modulus ..................................................151

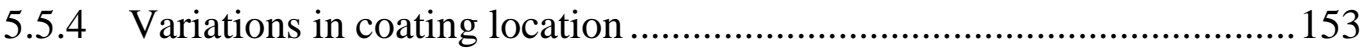

5.6 Development of a strain-rate dependent Mooney-Rivlin model for polyurea.

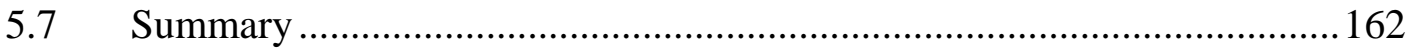

\section{Chapter 6 Summary and Recommendations ........................................................164}

6.1 Chapter 2: Literature review and identification of knowledge gaps..........164

6.2 Chapter 3: Preliminary experiments and numerical validation...................164

6.3 Chapter 4: Explosive testing of polyurea-coated steel plates .....................165 
6.4 Chapter 5: Numerical simulation and parametric studies ........................166

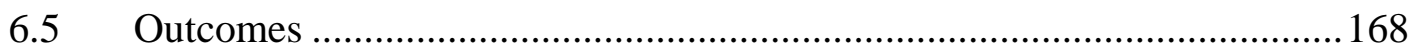

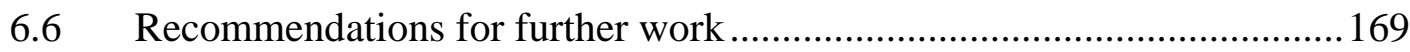

6.6.1 Implementation of the strain-rate dependent 5-parameter Mooney-Rivlin

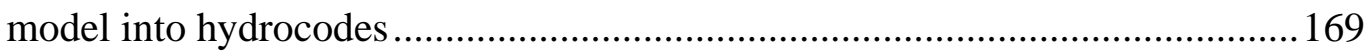

6.6.2 Further investigation into bond strength ..............................................169

6.6.3 Polyurea to prevent plate rupture ........................................................170

6.6.4 Polyurea to protect against ballistic penetration .................................170

6.6.5 Polyurea combined with other materials............................................170

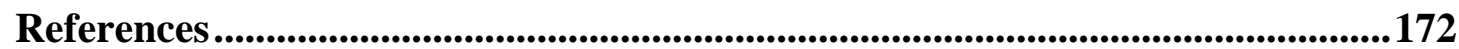

Publications Originating from this Thesis...........................................................180

Appendix A Blast Rig Drawings ............................................................181

Appendix B Pressure Gauge Results .........................................................184

Appendix C Plate Profiles Measured Using the 3D Laser Scanner..............194

Appendix D Tensile Test Data.....................................................................197

Appendix E International Journal of Impact Engineering Paper.................199 


\section{List of Figures}

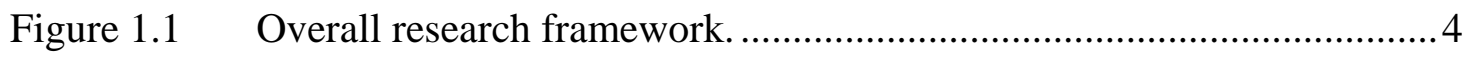

Figure 2.1 Typical pressure - time curve for an explosive blast wave (Baker et al., 1983).

Figure 2.2 Example of a typical measured blast wave overpressure history from a DSTO field trial, measured just outside the maximum fireball radius using a $\mathrm{PCB}^{\circledR}$ piezoelectric pressure gauge sampling at $5 \mathrm{MHz}$.

Figure 2.3 Blast regimes as defined by Ritzel (2007). 14

Figure 2.4 Example of plate petalling failure of a metallic plate (from a DSTO field trial).

Figure 2.5 Schematic of a mine blast protection system. If the lower floor does not impact the upper floor, the occupants will incur minimal loading. 16

Figure 2.6 The effect of stoichiometry (86, 91, 96, 101 and 106\%) on polyurea stress-strain behaviour. The inset shows toughness as a function of stoichiometry (Roland et al., 2007).

Figure 2.7 Stress-strain curves for a 96\% stoichiometry polyurea at various strain rates (Roland et al., 2007).

Figure 2.8 Sandwich plate designs with and without a polyurea interlayer (Bahei-El-Din and Dvorak, 2007).

Figure 2.9 Schematic view of the reverse ballistic test experimental setup (Amini et al., 2010b).

Figure 2.10 Four noded shell element with $3^{\text {rd }}$ order Gaussian integration, shown with a local coordinate system $(\xi, \eta, \zeta)$

Figure 2.11 Eight noded hexahedral element with one Gaussian integration point, shown with a local coordinate system $(\xi, \eta, \zeta)$.

Figure 2.12 Typical shapes of Mooney-Rivlin stress-strain curves. 35 
Figure 3.1 Charge and plate setup of the validation experiments from Ackland et al. (2007).

Figure 3.2 Flat specimen pre-test (left); deformed specimen after testing (right). The stand-off was varied between the experiments.

Figure 3.3 Experimental setup for the July 2007 Experiments.

Figure 3.4 Electrical schematic for the measurement of the plate transient deformation. The switches represent the plate (which was grounded) contacting each wire, $\mathrm{W}_{\mathrm{n}}$, in turn during its deformation stage, dropping the potential to approximately zero.

Figure 3.5 Wire deformation measurement array as set up before the blast event.

Figure 3.6 Example of a coated plate after the experiment (test V5).

Figure 3.7 Deformation wire results for test V1. The horizontal lines represent the wire locations.

Figure 3.8 Deformation wire results for test V2. The horizontal lines represent the wire locations.

Figure 3.9 The initial 1D Euler wedge setup used to model pentolite detonation as a function of radius ( $\mathrm{mm}$ ) from the detonation point (red diamond).

Figure 3.10 3D Euler model setup for an air-blast on a steel plate in AUTODYN, for test V3 (mesh elements not shown here). The dimensions are in millimetres and refer to the centre of detonation $(60.5 \mathrm{~mm})$ and the mesh boundaries, with the origin located at the centre of the steel plate.

Figure 3.11 Fitted stress strain data using a 2-parameter Mooney-Rivlin fit compared with experimental data from Roland et al. (2007). .50

Figure 3.12 Two of the meshes used for the mesh resolution study of a steel plate under impulsive loading. The coarsest mesh is shown on the left, and the finest mesh on the right. The blue diamonds indicate 
gauge positions. A velocity of $150 \mathrm{~m} / \mathrm{s}$ was applied to the nodes within the red dashed line.

Figure 3.13 Effect of mesh resolution on the peak residual deformation of a plate under impulsive loading using shell elements.

Figure 3.14 Effect of mesh resolution on the peak residual deformation of a plate under impulsive loading using hex elements (4 elements through thickness).

Figure 3.15 Meshes used to compare the effect of a weighted mesh (left) compared to an even mesh (right). The blue diamonds indicate gauge positions. A velocity of $200 \mathrm{~m} / \mathrm{s}$ was applied to the nodes within the red dashed line

Figure 3.16 Comparison of deformation histories for a weighted mesh (right) compared with an even mesh (left), using shell elements.

Figure 3.17 Comparison of deformation histories for a weighted mesh (right) compared with an even mesh (left), using hex elements.

Figure 3.18 Geometry change of an ideal element under a pure bending moment.

Figure 3.19 Geometry change of a fully integrated first-order element under a bending moment.

Figure 3.20 Effect of polyurea hex element size on peak residual plate deformation. The ' $X$ ' shows the result for a $2.6 \mathrm{~mm} \times 2.6 \mathrm{~mm}$ $\times 1 \mathrm{~mm}$ element size.

Figure 3.21 Effect of polyurea hex element dimensions on peak residual plate deformation.

Figure 3.22 3D Euler model of air-blast after remapping pentolite detonation (mesh elements not shown here). Dimensions are in mm.

Figure 3.23 Weighted mesh investigated to model air-blast. Dimensions are in $\mathrm{mm}$.

Figure 3.24 Peak reflected pressures measured along the reflecting surface for an air-blast, modelled using AUTODYN 
Figure 3.25 Mesh for air-blast on steel plate. The red line represents the location of the plate. In the solid green areas the mesh is too fine for the picture resolution.

Figure 3.26 Material energy summary for blast loading on a steel plate 66

Figure 3.27 AUTODYN model of an Eulerian air-blast interaction with a Lagrangian steel plate at approximately $0.05 \mathrm{~ms}$ after detonation.

Figure 3.28 Deformation histories for a $4 \mathrm{~mm}$ steel plate under blast loading measured using gauges in AUTODYN. The top plot was generated from a model using shell elements and the bottom plot was generated using hex elements.

Figure 3.29 Residual deformation of a bare steel plate under blast loading modelled using shell elements in AUTODYN

Figure 3.30 Residual deformation of a bare steel plate under blast loading modelled using hex elements in AUTODYN.

Figure 3.31 Comparison of numerically calculated residual deformation profiles with the experimental result (test V1).

Figure 3.32 Comparison between AUTODYN and experimental deformations for test V1

Figure 3.33 Setup for model of a polyurea-coated steel plate in AUTODYN. .73

Figure 3.34 Photographs of the uncoated plate V3 (top), the plate with $10 \mathrm{~mm}$ thick coating V5 (middle), and the plate with $19 \mathrm{~mm}$ coating V6 (bottom), showing contour lines from numerical models overlaid on experimental targets.

Figure 4.1 New blast test rig (not showing top plate or bolts). The total mass of the test rig including the top plate and bolts was approximately $640 \mathrm{~kg}$.

Figure 4.2 The polyurea coatings were applied over an area of $670 \mathrm{~mm} \times 670 \mathrm{~mm}$ to fit within the blast rig, which had a free-span area of $700 \mathrm{~mm} \times 700 \mathrm{~mm}$.

Figure 4.3 Pre-event experimental setup. .82 
Figure 4.4 Flat specimen pre-test (left); Deformed specimen after testing (right)

Figure 4.5 Blast rig internal setup showing the instrumentation for transient deformation measurement.

Figure 4.6 Still image of the scale taken using the high speed camera. The same resolution was used for the high speed video imaging (624 $\times 112$ pixels). The pixel size allowed a discretisation of $0.5 \mathrm{~mm}$ in the scale.

Figure 4.7 Setup inside the test rig showing the mechanical probe positioned with its tip against the plate centre.

Figure 4.8 Pressure history recorded by gauge P1 at 4 m during Event 4 ...............89

Figure 4.9 Post-event deformation of a bare 6 mm thick steel plate (plate B1).....90

Figure 4.10 Post-event deformation of a $5 \mathrm{~mm}$ thick steel plate with a $7.7 \mathrm{~mm}$ polyurea coating (plate C3), showing de-bonding over a circular area at the centre of the plate (bordered by the dashed line).

Figure 4.11 Post-event deformation of a $4 \mathrm{~mm}$ thick steel plate with a $15.7 \mathrm{~mm}$ thick polyurea coating (plate D1), showing de-bonding reaching the polyurea edge. The polyurea remained joined to the plate at the other three corners.

Figure 4.12 Post-event deformation of a $4 \mathrm{~mm}$ thick steel plates with a $15.7 \mathrm{~mm}$ thick polyurea coating (plate D2) showing tearing of the polyurea near the plate centre. Again, the polyurea remained joined to the plate at three of the corners.

Figure 4.13 Bare $6 \mathrm{~mm}$ steel plate showing rupture after Event 7 (plate B3), in which a mechanical probe was positioned below the plate

Figure 4.14 Effect of coating thickness on peak residual plate deformation............95

Figure 4.15 Effect of charge mass on peak residual plate deformation...................96

Figure 4.16 Effect of plate temperature on peak residual plate deformation. 97

Figure 4.17 Still images recorded by the Phantom Miro-3 high speed camera during Event 8 (plate B4) at 0.12 ms intervals. The first image 
shows the image of the scale taken before the plate was attached to the test rig.

Figure 4.18 Still images recorded by the Phantom Miro-3 high speed camera during Event 9 at $0.12 \mathrm{~ms}$ intervals (plate C3). The first image shows the image of the scale taken before the plate was attached to the test rig

Figure 4.19 Still images recorded by the Phantom Miro-3 high speed camera during Event 10 at $0.14 \mathrm{~ms}$ intervals. The first image shows the image of the scale taken before the plate was attached to the test rig.

Figure 4.20 Deformation history determined from high speed video footage of Event 8 (plate B4), Event 9 (plate C3) and Event 10 (plate D3).........102

Figure 4.21 Curve fit to the ratio of polyurea deformation in Event 10 to Event 9.

Figure 4.22 Curve fit used to predict the peak deformation of plate C3 (Event 9).

Figure 4.23 Pink spray paint was applied to the plate boundaries to indicate the amount of slippage that occurred during each event (pre-event image).

Figure 4.24 The left hand image shows the plate boundary before an event. The right hand image shows the plate boundary after the event, with plate boundary movement as indicated.

Figure 5.1 Eulerian mesh used to model air-blast on plates. The solid green areas contain elements too small to be distinguished due to the resolution of the image. Dimensions are in $\mathrm{mm}$

Figure 5.2 Dimensions used for tensile test specimens as per ASTM standard E 8/E 8M-08.

Figure 5.3 Mesh used for modelling polyurea de-bonding from the plate during the blast. The orange area represents the polyurea and the pink area shows the outer edge of the steel plate. 
Figure 5.4 Through thickness mesh showing the polyurea (in orange) and the bond material (in light blue). The steel plate is not shown here.

Figure 5.5 Polyurea-coated plate model showing the initiation of polyurea debonding. The light blue region represents the void caused by local de-bonding.

Figure 5.6 Results of numerical modelling showing the effect of the bond failure stress on the radius over which the polyurea de-bonds

Figure 5.7 Polyurea de-bonding for the $5 \mathrm{~mm}$ steel plate with a $7.7 \mathrm{~mm}$ thick polyurea coating. The light blue region represents the void caused by local de-bonding.

Figure 5.8 Polyurea de-bonding (continued) for the $5 \mathrm{~mm}$ steel plate with a $7.7 \mathrm{~mm}$ thick polyurea coating. The polyurea is shown to "spring back”, coming to rest against the plate.

Figure 5.9 Polyurea de-bonding for the $4 \mathrm{~mm}$ steel plate with a $15.7 \mathrm{~mm}$ thick polyurea coating. The light blue region represents the void caused by local de-bonding.

Figure 5.10 Polyurea de-bonding (cont.) for the $4 \mathrm{~mm}$ steel plate with a $15.7 \mathrm{~mm}$ thick polyurea coating. The bond is shown to fail across the width of the plate at the sectioned position (from $2.5 \mathrm{~ms}$ ).

Figure 5.11 Element distortion of the de-bonded polyurea for the $5 \mathrm{~mm}$ plate with the $7.7 \mathrm{~mm}$ thick coating.

Figure 5.12 Comparison of the experimental plate deformation profile and numerical modelling result for the uncoated $6 \mathrm{~mm}$ steel plate (Plate B4).

Figure 5.13 Comparison of the experimental plate deformation profile and numerical modelling result for the $5 \mathrm{~mm}$ steel plate with a polyurea coating (Plate C3).

Figure 5.14 Comparison of the experimental plate deformation profile and numerical modelling result for the $4 \mathrm{~mm}$ steel plate with a polyurea coating (Plate D3). 
Figure 5.15 Comparison of high speed video data with AUTODYN predictions for the deformation at the plate centre of an uncoated $6 \mathrm{~mm}$ steel plate (B4) under blast loading.

Figure 5.16 Comparison of experimental and numerical results for deformation at the plate centre.

Figure 5.17 Mooney-Rivlin fit to polyurea stress-strain data from Roland et al. (2007), with the marker showing the approximate peak strain and strain rate from the numerical model.

Figure 5.18 Tensile specimen dimensions (Lu, 2011).........................................133

Figure 5.19 Tensile specimens used for the initial tests.

Figure 5.20 Tensile test specimen in the Instron 8800 test machine. The left image shows the specimen before testing, and the right hand image shows the specimen after testing

Figure 5.21 Results of quasi-static and dynamic tensile tests on polyurea.

Figure 5.22 Linear curve fit to experimental data from dynamic high strain rate tensile tests at $10 \mathrm{~s}^{-1}$

Figure 5.23 2-parameter Mooney-Rivlin curve fit to high strain rate tensile test results at $10 \mathrm{~s}^{-1}$ using constants $C_{01}=-1461.1 \mathrm{kPa}$ and $C_{10}=9077.6 \mathrm{kPa}$.

Figure 5.24 Linear curve fit to experimental data from dynamic high strain rate tensile tests at $10 \mathrm{~s}^{-1}$ with data points removed for strains less than 0.5

Figure 5.25 2-parameter Mooney-Rivlin curve fit to high strain rate tensile test results at $10 \mathrm{~s}^{-1}$ using constants $C_{01}=5568 \mathrm{kPa}$ and $C_{10}=43.9 \mathrm{kPa}$.

Figure 5.26 Comparison of the experimental plate deformation profile and numerical modelling result for the $5 \mathrm{~mm}$ steel plate with a polyurea coating (Plate C3), using new Mooney-Rivlin constants. 
Figure 5.27 Comparison of the experimental plate deformation profile and numerical modelling result for the $4 \mathrm{~mm}$ steel plate with a polyurea coating (Plate D3), using new Mooney-Rivlin constants.

Figure 5.28 Comparison of experimental and numerical results for the deformation of the plate centre, using Mooney-Rivlin constants $C_{01}=5568 \mathrm{kPa}$ and $C_{10}=43.9 \mathrm{kPa}$.

Figure 5.29 Effect of bond strength on the peak transient deformation of the polyurea coating.

Figure 5.30 Effect of bond strength on the radius of the polyurea de-bonded area.

Figure 5.31 Effect on the stress strain curve from varying the Mooney-Rivlin material constants $C_{01}$ and $C_{10}$ to double the initial stiffness.

Figure 5.32 Stress strain plots using different Mooney-Rivlin constants.

Figure 5.33 Effect of polyurea stiffness on the peak transient deformation of the polyurea.

Figure 5.34 Effect of polyurea stiffness on the radius of the de-bonded area........151

Figure 5.35 Effect of polyurea bulk modulus on peak transient deformation. 152

Figure 5.36 Effect of polyurea bulk modulus on the radius of the de-bonded area.

Figure 5.37 Two methods of defining the stand-off for front face coatings 155

Figure 5.38 Simulation of the blast response of a steel plate with a polyurea coating on the front face.

Figure 5.39 Simulation of the blast response of a plate with a steel/polyurea/steel sandwich configuration.

Figure 5.40 Strain-rate dependent 5-parameter Mooney-Rivlin curve fits to experimental tensile test data of polyurea. 


\section{List of Tables}

Table 3.1 Test plate mechanical properties (Bluescope Steel, 2009, MIL-S22698C, 1988).

Table 3.2 Results of the validation experiments.

Table $3.3 \quad$ Johnson-Cook material model parameters used to model D36 steel (Balden and Nurick, 2005).

Table 3.4 Numerical modelling results for the deformation of the plate centre compared with experimental results. 70

Table $3.5 \quad$ Numerical modelling results of polyurea-coated D36 steel plate results compared to experimental from Ackland et al. (2007). .74

Table 4.1 Test plate details. All test plates had an areal density of $4.7 \mathrm{~g} / \mathrm{cm}^{2} \ldots . . .81$

Table 4.2 Instrumentation summary. The ticks indicate devices which were used and the crosses indicate devices which weren’t used.

Table 4.3 Residual deformation results for the blast experiments. All the target plates had a total areal density of $4.7 \mathrm{~g} / \mathrm{cm}^{2}$ over the test area....93

Table 4.4 Average residual deformation results for the blast experiments...........94

Table 4.5 Peak deformation values calculated from the high speed video. .........104

Table 5.1 Results of tensile tests on the XLERPLATE 350 steel. .......................113

Table 5.2 Residual deformation results of the numerical modelling...................124

Table 5.3 Comparison of numerical modelling results using two different Mooney-Rivlin material models for polyurea 


\section{List of Symbols and Abbreviations}

1D

3D

A

AFRL

B

B

B1, B2, B3, B4

C

C

$C_{01}$

$C_{1}$

C1, C2, C3

$C_{10}$

$C_{2}$

CFD

CPU

$d$

D1, D2, D3

DSTO

E

$e_{\text {int }}$

ERDC

EVE
One dimensional

Three dimensional

Length of reduced section (tensile test specimen dimension)

Air Force Research Laboratory

Length of grip section (tensile test specimen dimension)

Johnson-Cook strength model material dependent parameter

Experimental plate numbers (6 mm bare plates)

Width of grip section, approximate (tensile test specimen dimension)

Johnson-Cook strength model material dependent parameter

Empirically determined constant for Mooney-Rivlin material model

Empirically determined constant for JWL material model

Plate numbers (5 mm plates with nominally $7.7 \mathrm{~mm}$ coatings)

Empirically determined constant for Mooney-Rivlin material model

Empirically determined constant for JWL material model

Computational fluid dynamics

Central processing unit

Material incompressibility parameter

plate numbers (4 mm plates with nominally $15.7 \mathrm{~mm}$ coatings)

Defence Science and Technology Centre

Tensile modulus

Internal energy

Engineer Research and Development Center

E-glass vinyl ester 


\begin{tabular}{|c|c|}
\hline G & Gauge length (tensile test specimen dimension) \\
\hline$G$ & Shear modulus \\
\hline hex & hexahedral \\
\hline $\bar{I}_{1}$ & $\begin{array}{l}\text { First invariant of the deviatoric component of the left Cauchy-Green } \\
\text { deformation tensor }\end{array}$ \\
\hline $\bar{I}_{2}$ & $\begin{array}{l}\text { Second invariant of the deviatoric component of the left Cauchy- } \\
\text { Green deformation tensor }\end{array}$ \\
\hline IED & Improvised explosive device \\
\hline ISAF & International Security Assistance Force \\
\hline ISO & International Organisation for Standardisation \\
\hline$J$ & Determinant of the elastic deformation gradient \\
\hline JWL & Jones-Wilkins-Lee \\
\hline$K E$ & Kinetic energy \\
\hline $\mathrm{L}$ & Overall length (tensile test specimen dimension) \\
\hline LS-DYNA® & $\begin{array}{l}\text { Explicit, non-linear dynamics program used for the analysis of } \\
\text { dynamic structural response }\end{array}$ \\
\hline LVDT & Linear variable differential transformer \\
\hline$M$ & Mass \\
\hline$m$ & Johnson-Cook strength model material dependent parameter \\
\hline$n$ & Johnson-Cook strength model material dependent parameter \\
\hline NSWC-CD & Naval Surface Warfare Centre, Carderock Division \\
\hline$p$ & Static pressure \\
\hline P\&EE & Proof and Experimental Establishment \\
\hline$p_{0}$ & Ambient pressure \\
\hline $\mathrm{P} 1, \mathrm{P} 2, \mathrm{P} 3, \mathrm{P} 4$ & Pressure gauge numbers \\
\hline PEC & Prototype Engineering Centre (Laverton, Australia) \\
\hline$P_{s^{-}}$ & Minimum overpressure \\
\hline
\end{tabular}




\begin{tabular}{|c|c|}
\hline$P_{s}^{+}$ & Peak overpressure \\
\hline PU1 & Low tensile modulus polyurea \\
\hline PU2 & High tensile modulus polyurea \\
\hline$q$ & Dynamic pressure \\
\hline $\mathrm{R}$ & Radius of fillet (tensile test specimen dimension) \\
\hline$R$ & Static damping constant for optimal convergence \\
\hline$r_{1}$ & Empirically determined constant for JWL material model \\
\hline$r_{2}$ & Empirically determined constant for JWL material model \\
\hline RAM & Random access memory \\
\hline$R_{\mathrm{f}}$ & Fireball radius \\
\hline RTV & Room temperature vulcanising \\
\hline$s$ & Thermal softening exponent \\
\hline $\mathrm{T}$ & Thickness (tensile test specimen dimension) \\
\hline$T$ & Material temperature \\
\hline$t$ & Time \\
\hline$T$ & Negative phase duration \\
\hline$T^{+}$ & Positive phase duration \\
\hline$t_{a}$ & Time of arrival \\
\hline$T_{g}$ & Glass transition temperature \\
\hline$T_{\text {melt }}$ & Material melting temperature \\
\hline$T_{\text {room }}$ & Room temperature \\
\hline$V$ & Flow velocity \\
\hline $\begin{array}{l}\mathrm{V} 1, \mathrm{~V} 2, \mathrm{~V} 3, \\
\mathrm{~V} 4, \mathrm{~V} 5, \mathrm{~V} 6\end{array}$ & Plate numbers ( $4 \mathrm{~mm}$ bare plates) \\
\hline $\mathrm{W}$ & Width (tensile test specimen dimension) \\
\hline$\alpha$ & Angle used in shear locking description \\
\hline & Adiabatic exponent \\
\hline
\end{tabular}




$\begin{array}{ll}\Delta t & \text { Time step } \\ \varepsilon & \text { Engineering strain } \\ \dot{\bar{\varepsilon}}_{0} & \text { Reference strain rate } \\ \bar{\varepsilon}_{p} & \text { Equivalent plastic strain } \\ \dot{\bar{\varepsilon}}_{p} & \text { Normalised equivalent plastic strain rate } \\ \kappa & \text { Bulk modulus } \\ \lambda & \text { Stretch ratio } \\ \mu & \text { ( } / \rho_{0} \text { ) - 1 } \\ \rho & \text { Density } \\ \rho_{0} & \text { Reference density } \\ \bar{\sigma} & \text { Yield stress at non-zero strain rate } \\ \sigma_{0} & \text { Yield stress at low strain } \\ \omega & \text { Strain energy function } \\ \omega & \text { Empirically determined constant for JWL material model (adiabatic } \\ & \text { constant) } \\ & \end{array}$




\section{Chapter 1}

\section{Introduction}

\subsection{Background}

Australia’s current military contribution to the International Security Assistance Force (ISAF) in Afghanistan (deployed under Operation SLIPPER) includes an average of 1,550 Australian Defence Force personnel deployed within Afghanistan each year (Australian Government Department of Defence, 2012). The operations in Afghanistan have seen Improvised Explosive Devices (IEDs) as a prevalent threat, causing the deaths of 15 of the 39 Australian casualties (as of 3 December 2012), and over 50\% of coalition military deaths (Operation Enduring Freedom, 2012). These deaths have occurred to both mounted soldiers (i.e. in armoured vehicles) and dismounted soldiers. Due to the prevalence and lethality of IEDs, methods to increase the blast protection of vehicles are required. A common approach to increase the blast protection of a vehicle is to add more metallic armour; however, this can have a detrimental effect on vehicle mobility, dynamic stability, and payload capacity. It is for this reason that lightweight armour solutions are being sought to provide vehicle blast protection.

A number of studies have explored the use of polyurea coatings as a lightweight material which can be applied to structures to enhance their blast protection. In many cases, the polyurea is applied to concrete masonry wall units (e.g. Davidson et al., 2005, Davidson and Sudame, 2004, Knox et al., 2000) and acts to prevent fragments breaking off the back surface of the wall, effectively holding it together during the blast event. Polyurea has also been used in the design of sandwich materials (BaheiEl-Din and Dvorak, 2007, Bahei-El-Din et al., 2006, Gardner et al., 2012), composite panels (Hui and Oskay, 2012, Kim et al., 2012, McShane et al., 2008, Tekalur et al., 2008) and as a helmet suspension pad material (Grujicic et al., 2007), to mitigate blast effects. Additionally, some researchers have considered the use of polyurea to 
improve the blast or impact load resistance of metallic structures (Ackland et al., 2007, Amini et al., 2010a, Amini et al., 2006, Amini et al., 2010b, Matthews, 2004, McShane et al., 2008). In the case of polyurea-coated steel plates, the mechanisms by which the polyurea coatings protect against blast loading are not fully understood.

\subsection{Problem description and research questions}

Although results (e.g. Ackland et al., 2007, Amini et al., 2010a, Amini et al., 2006, Amini et al., 2010b, Matthews, 2004, McShane et al., 2008) indicate that polyurea coatings can enhance the resistance of metallic structures to deformation and failure under blast loads, there is limited information available on how this is achieved. This means that these coatings may be applied to existing metallic structures or incorporated into the design of new structures without a thorough understanding of whether they will enhance or degrade blast protection. Furthermore, existing research primarily investigates polyurea coatings as appliqués, without considering their incorporation into the blast protective design of structures. Before polyurea coatings can be responsibly applied to metallic surfaces to enhance blast protection, either as an appliqué or in vehicle or structural design, a better understanding of their behaviour needs to be developed. The work in this thesis aims to provide a better understanding of how polyurea-coated plates behave under blast loading, and how their properties may be modified to improve their performance.

The main research questions that were considered during this research project are summarised below.

\section{How effective are polyurea-coated steel plates at reducing deformation under blast loading compared to homogeneous steel plates of equivalent mass?}

Polyurea coatings have previously been shown to enhance the blast resistance of metallic structures when applied as an appliqué. The addition of a polyurea coating to an existing structure is advantageous because a polyurea coating can easily be applied in retrofit to the structure. However, their advantage as an inclusion in the design stages of a vehicle or structure is unclear. This research investigates whether it would be beneficial to include polymer coatings at the design stage of a metallic structure for 
blast protection, or if it would be better to use a metallic solution of equivalent mass. It could be argued that a simplified quasi-static analysis would lead to the conclusion that polyurea-coated plates will deform more than steel plates of equivalent areal density, due to decreased bending stiffness. However, the behaviour of these materials under blast loading is difficult to predict analytically. High deformation, localised blast loading results in a time-dependent, non-linear scenario where the plates undergo large plastic deformations. Furthermore, both steel and polyurea exhibit strain and strain-rate dependent behaviour. For these reasons, experimentally validated numerical simulations are needed to evaluate the comparative performance.

\section{What are the properties of polyurea that affect the performance of polyurea- coated plates under blast loading?}

Little is known about the properties that allow polyurea coatings to modify the blast resistance of metallic structures. For example, the properties of polyurea change at high strain rates. This behaviour can be explored through mechanical testing, which can then be used to develop material models to simulate the response to blast loading. The use of experimentally-validated numerical models of polyurea-coated plates under blast loading allows properties such as polyurea stiffness and bulk modulus to be explored, without the need to formulate new materials. If an improved understanding of how polyurea properties affect the performance of polyurea-coated plates under blast loading is developed, future polymers could be custom designed to better enhance their effectiveness against blast.

\section{What other factors contribute to the performance of polyurea-coated plates under blast loading?}

In addition to the properties of polyurea itself, the manner in which it is applied to metallic structures is likely to affect how the structure performs against blast. This includes factors such as the bond strength and the face to which the coating is applied. A better understanding of these factors will lead to improved applications of such coatings in the future. As with the second research question, the development of validated numerical models is invaluable in conducting such studies. 


\subsection{Research framework and scope}

The primary aim of the research was to investigate the effect of polyurea coatings on the blast performance of steel plates under blast loading. The overall structure of the research used to achieve this aim can be represented using the framework shown in Figure 1.1.

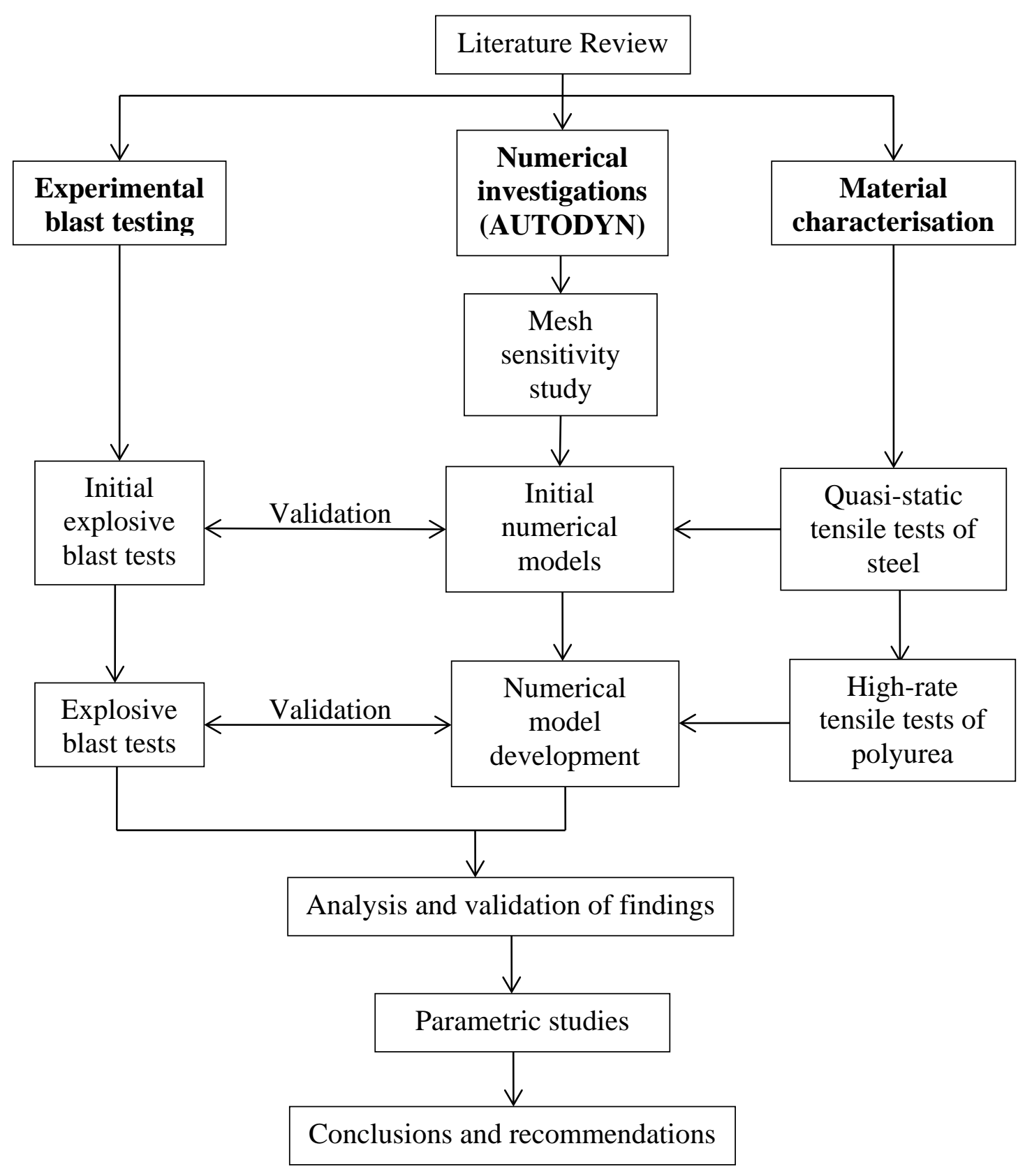

Figure 1.1 Overall research framework. 
The research began with an assessment of the state of the art in explosive testing and numerical modelling. This allowed the identification of knowledge gaps and a research plan to be constructed. As shown in Figure 1.1, the main bodies of research can be separated into three general categories: experimental blast testing, numerical investigations, and material characterisation. These research areas were conducted in parallel to encourage a timely completion of the work. Once the experimental testing and numerical model development were complete, the results were analysed and parametric studies commenced. The parameters were selected based on the results of the previous numerical and experimental studies. Finally, the collated results of the studies allowed conclusions to be drawn and recommendations to be made.

A single type of steel was selected as the base metallic material for this research, to allow the focus to remain on the polyurea behaviour. However, the experimental and numerical methods developed here could be applied to different metals in future studies. A single polyurea composition was used throughout the experimental program; however variations in its properties were investigated numerically. The constitutive behaviour and mechanical properties of the polymer at quasi-static and higher strain rates in tension were initially sourced through literature. However, during the course of the project it was identified that in-house testing was required to develop more accurate material models.

Numerical modelling was conducted using the hydrocode ANSYS ${ }^{\circledR}$ AUTODYN ${ }^{\circledR}$ (2009). This explicit code was selected over an implicit code due to its improved convergence properties in highly nonlinear rate-dependent problems. It was also selected for its ability to conduct coupled Euler-Lagrange simulations which are essential for numerical modelling of the large-deformation response of plates to blast loading. The decision to use AUTODYN was complemented by the Candidate's prior experience and training in using the code. The validated numerical models were used as the foundation for parametric studies to investigate the effects of bond strength between the polyurea and the plate, polyurea stiffness, polyurea bulk modulus, and polyurea location with respect to the plate (front, back or sandwich configuration).

Close-range, localised, explosive blast loading was considered the loading case most relevant to vehicles subjected to underbelly IED threats. In this regime, the 
momentum transfer from the expanding explosive detonation products dominates the loading, as opposed to far-field blast loading which is dominated by the air shock.

In this research program, plate deformation (transient and residual) was used as the measure of performance. Plate rupture was not considered in this program, as reducing deformation (without rupture) is of prime importance for underbelly IED protection systems. However, preventing rupture can also be important and is identified as a recommended area for future research.

\subsection{Thesis structure}

The initial part of this research comprised of an assessment and critical review of the current literature, which is presented in Chapter 2. The literature review covers the basics of blast wave formation and blast regimes, before moving on to the effect of blast loading on plates, and the current knowledge in the area of polyurea coatings for blast protection. Following this is a discussion on methods for numerical simulation of blast loading, structural response and material behaviour.

Chapter 3 describes the next stage of the research, which was to develop experimental methods for explosive testing of plates, and to validate preliminary numerical models of polyurea-coated plates under blast loading. Experimental blast tests were conducted against bare steel plates, and further blast test results for polyurea-coated steel plates were sourced from literature. The numerical models incorporated material models for steel and polyurea which were sourced from literature, and used a coupled EulerLagrange simulation to model the interaction between the blast loading and the plate. Mesh refinement studies were conducted for all the Euler and Lagrange parts, to ensure the spatial discretisation was sufficient to capture the loading and plate deformations. Two different element types for the steel were also investigated. The models, as well as the experimental procedures, provided a basis for the subsequent experimental and numerical research.

Chapter 4 details the explosive blast testing of polyurea coated steel plates. These experiments were conducted to determine whether the application of polyurea coatings to steel plates can reduce their deformation under blast loading, compared to 
uncoated steel plates with equivalent areal densities. The experiments also investigated the effect of the polyurea to steel ratio for mass-equivalent plates, and the test results provided data for validation of numerical models. A new test rig was designed and manufactured for the experiments, incorporating instrumentation for capturing the plate and polyurea transient deformation. The deformation measurements, both transient and residual, were used for validation of the numerical models in Chapter 5. The test methodology and measurement techniques were designed to be reproducible and adaptable should future testing of different plate materials be required.

Chapter 5 covers the numerical modelling of the experiments and the parametric studies. The model applied in this chapter used the specific polyurea from the experiments and enabled de-bonding between the polyurea coating and the steel plate. Tensile tests were conducted on the polyurea at various strain rates to derive the two-parameter Mooney-Rivlin constants for the Versalink P1000/Isonate 143L polyurea.

Parametric studies were conducted using the validated numerical model with de-bonding and updated polyurea Mooney-Rivlin constants. The variables considered in the parametric studies were: the polyurea stiffness and bulk modulus; the bond strength between the polyurea and the plate; and the coating location (front face coating, back face coating and a sandwich configuration). These studies investigated how the physical properties of the polyurea-coated plates or the mechanical properties of polyurea can be varied to affect the performance under blast loading. Such information can be used to improve the blast performance of polyurea-coated steel structures in the future.

In Chapter 6, the major conclusions of the research are summarised, and recommendations for future work are provided. 


\section{Chapter 2}

\section{Literature Review}

This literature review was conducted to evaluate the current knowledge in areas relating to blast loading and response of polyurea-coated plates. The first section covers some blast physics principles, including the formation of blast waves, and definitions of loading regimes and their effect on structural response. The second section covers the response of plates to blast loading, the key mechanisms of blast protection and how polyurea coatings may affect such protection. The third section covers numerical simulation of blast loading, plate response, and polyurea coatings.

\subsection{Blast physics}

This section discusses the propagation of a blast wave in air as a result of a detonated explosive.

\subsubsection{Pressure}

Pressure arises from the movement of molecules within a medium and their interaction with their surroundings. When molecules of a medium interact with their surroundings, such as the wall of a container, they change direction, hence imparting momentum to the walls. This causes a force on the wall, which on a larger scale can be measured as a total force over an area (Çengel and Boles, 1994). By definition, pressure is force per unit area.

Under flow conditions, such as those experienced in a blast situation, we may consider two types of pressure, namely static and dynamic pressure. 


\subsubsection{Static pressure}

The static pressure is the pressure caused by the random movement of molecules in a fluid, as described previously. It is measured perpendicular to the flow direction, and is the pressure that you would experience if you were moving along with the flow. When pressure is mentioned in the literature it is usually the static pressure, unless otherwise stated. This convention is used throughout this thesis.

\subsubsection{Dynamic pressure}

Pressure can also be caused by a directional, ordered motion when the particles of the medium are flowing. Because the motion is directional, a component of the total pressure is also directional. The pressure caused by the directional component of motion is the dynamic pressure. In a blast wave this is the pressure resulting from the high velocity wind behind the shock front, which is essentially the kinetic energy per unit volume of air immediately behind the shock front (Beveridge, 1998). The kinetic energy $K E$ for a mass $M$ and flow velocity $V$ is given by:

$$
K E=\frac{1}{2} M V^{2}
$$

If we divide the kinetic energy by a unit volume, then we have the dynamic pressure $q$ (where $\rho$ is the density of the ambient medium):

$$
q=\frac{1}{2} \rho V^{2}
$$

\subsubsection{Absolute pressure and gauge pressure}

The absolute pressure is the static pressure measured at a given position relative to absolute vacuum, i.e. absolute zero pressure (Çengel and Boles, 1994). However, most gauges are calibrated such that they read zero at atmospheric pressure. The difference between the absolute pressure and atmospheric pressure is known as the gauge pressure. According to the International Organisation for Standardisation (ISO) (International Organization for Standardization, 1975), the standard atmospheric pressure at sea level is $101.325 \mathrm{kPa}$. 


\subsubsection{Overpressure}

Overpressure is a term that is often used when describing blast pressures. It is equal to the gauge pressure.

\subsubsection{Reflected pressure}

The reflected pressure of a blast wave is the pressure measured on a non-responding reflecting surface, caused by the reflection of the blast wave. It is a combination of the pressure acting on the surface due to the arrest and/or deflection of the dynamic flow, and the static pressure.

\subsubsection{Shock waves}

A shock wave is a very high pressure disturbance which moves at or above the speed of sound of the surrounding medium. The leading edge of a shock wave is known as a shock front, across which there is an almost instantaneous rise in pressure, density, particle velocity and internal energy. Behind the shock front there is a decay in these variables, which will eventually return to the ambient conditions.

\subsubsection{Blast wave formation}

If we consider a spherical charge centrally initiated under free air conditions, then a blast wave is generated when the atmosphere surrounding the detonated explosive is forcibly pushed outward by the expanding detonation products. The blast wave is comprised of the expanding detonation products (also known as the fireball), and a shock wave with its front at the leading edge of the blast wave. Initially, the fireball and the shock wave have the same radius. As the expansion continues, the mass of the detonation products cause them to slow down relative to the shock front, and the shock front separates from the fireball. Gayle and Bransford (1965) developed an equation to calculate the maximum fireball diameter, $\mathrm{R}_{\mathrm{f}}$ (in metres), from the detonation of a high explosive of mass $M$ (in kilograms), based on 14 experiments using TNT, C4 and pentolite. The equation is given by:

$$
R_{f}=3.39 M^{0.341}
$$


Blast waves are often defined by the static pressure or overpressure and time profile of the shock wave after it has separated from the fireball. The peak static overpressure and duration of the shock wave are dependent on the explosive type used, as well as the geometry of the charge and the method of initiation. Figure 2.1 provides a schematic representation of an idealised blast wave static pressure history measured at a constant distance from the centre of the charge. The time at which the shock front arrives at a measurement point is known as the time of arrival, $t_{a}$. The pressure rises almost instantaneously from ambient, $p_{0}$, to a peak pressure above ambient, $P_{s}{ }^{+}+p_{0}$, after which it will commence an almost instantaneous decay. The pressure decreases for a time $T^{+}$until it passes through ambient pressure, $p_{0}$. After this point there is a negative pressure phase, for time $T^{-}$, after which the pressure will return to ambient.

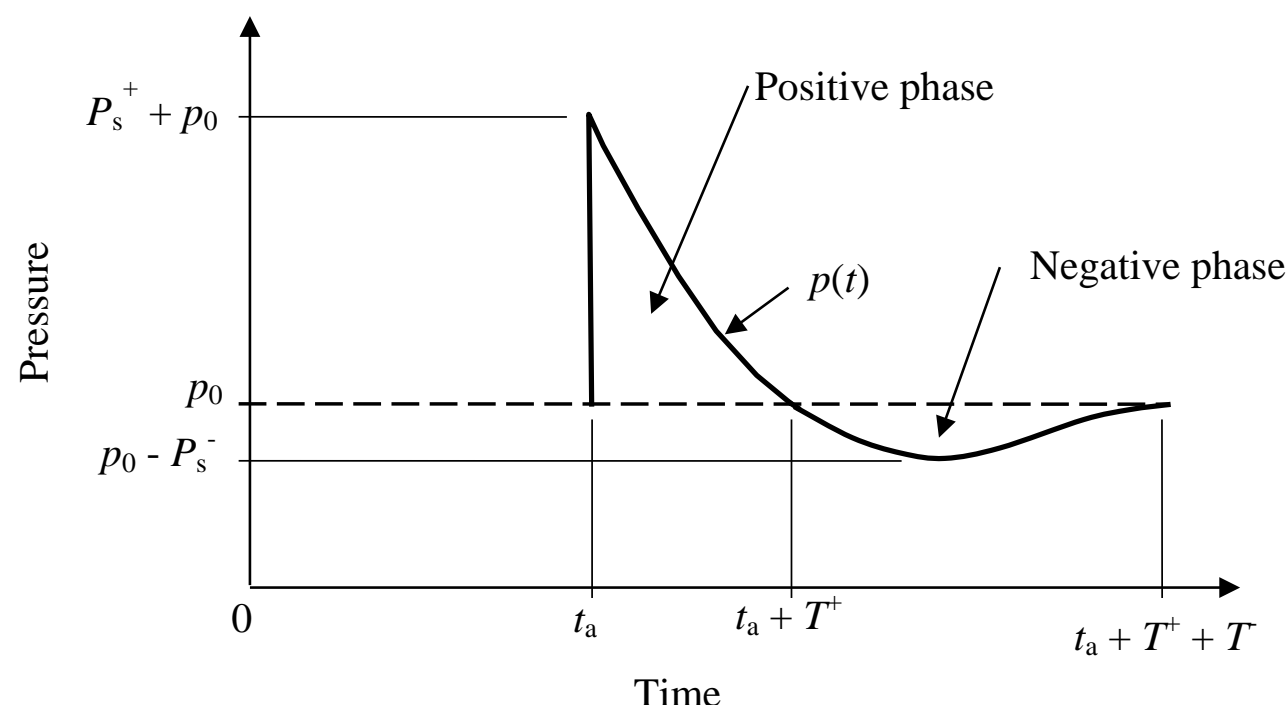

Figure 2.1 Typical pressure - time curve for an explosive blast wave (Baker et al., 1983).

An ideal profile resembling that shown in Figure 2.1 would typically be obtained at a large distance from a charge, well outside the fireball. However, at close range the blast wave pressure profile can be very different from the ideal profile. As an example, Figure 2.2 shows the static overpressure profile of a typical measured blast wave from a DSTO trial, taken at a location just beyond the fireball. Note that no 
negative phase is recorded and there is a large spike at the time of arrival, which is known as gauge overshoot. Gauge overshoot is an artificial measurement rather than a true measured property of the blast wave. According to Walter (2010), the structural resonance of the housed quartz transduction element used in many pressure gauges can be excited by the high frequency content of the shock front passing the sensing surface of the transducer, which in turn can cause gauge overshoot. There are methods available to extract a more realistic peak profiles containing noise and gauge overshoots using curve fitting methods, the most common for blast waves being the use of a Friedlander curve (Friedlander, 1946). However, care should be taken when using such curve fits for non-ideal blast measurements at close range.

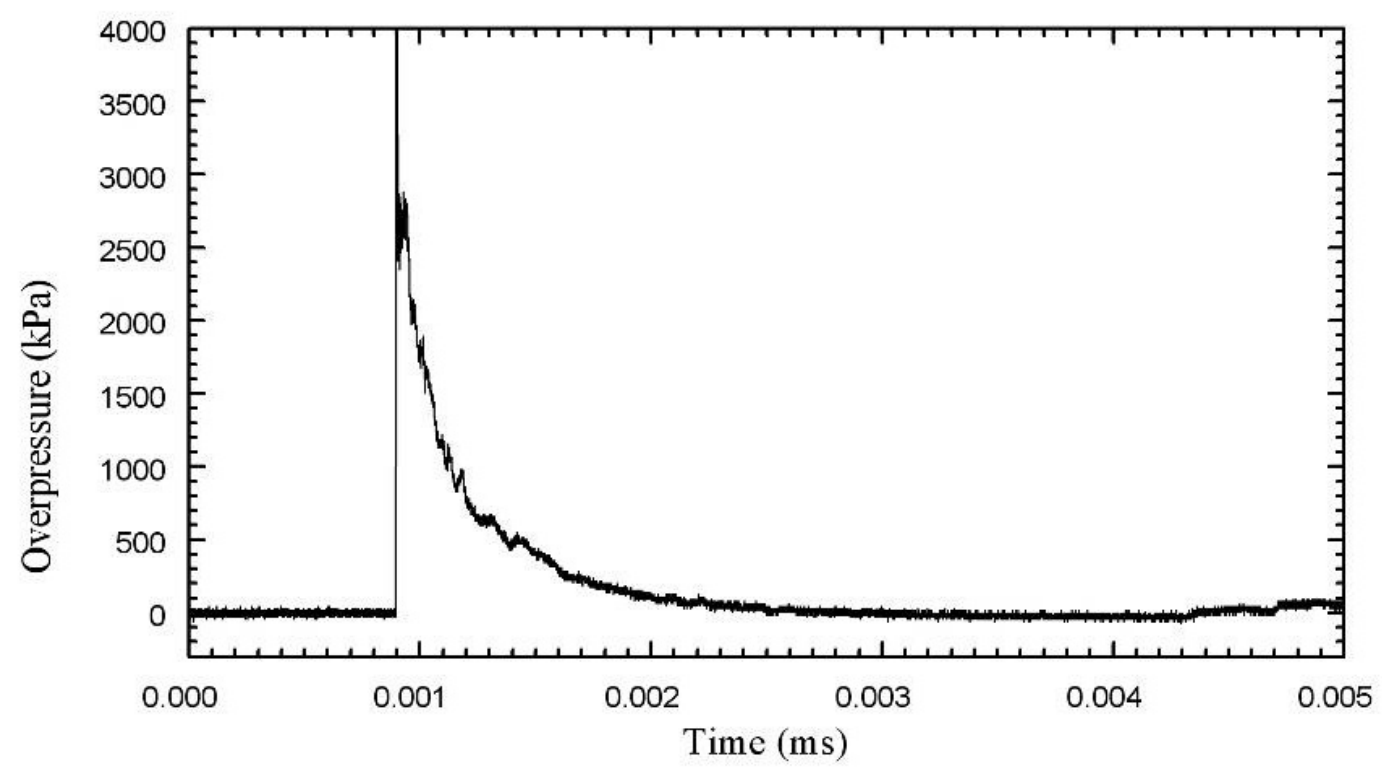

Figure 2.2 Example of a typical measured blast wave overpressure history from a DSTO field trial, measured just outside the maximum fireball radius using a $\mathrm{PCB}^{\circledR}$ piezoelectric pressure gauge sampling at $5 \mathrm{MHz}$.

\subsubsection{Blast effects on a structure}

The damage mechanisms of blast loading on a structure are heavily dependent on the distance from the explosive. Four regimes can be defined which relate the distance 
from the detonation to the target damage mechanisms (from Ritzel, 2007). These are shown schematically in Figure 2.3 and defined as follows:

Detonics Regime - The target is in direct or near contact with the explosive, typically less than $0.5 R_{\mathrm{f}}$ (where $R_{\mathrm{f}}$ is the radius of the fireball). The interaction with the target inside the fireball is dominated by the physical flow of detonation products. Most damage is inflicted by the expansion and kinetic energy of the detonation products.

Near-field Regime - The target is just beyond the fireball, typically less than $2 R_{\mathrm{f}}$. There is a complex shock structure with no negative pressure phase and multiple components (fireball and shock wave considerations). Numerical models must account for impingement of the fireball and detonation products in addition to the airshock phenomena.

Mid-field Regime $-2 R_{\mathrm{f}}$ to $10 R_{\mathrm{f}}$. The target encounters a non-uniform shock structure, single positive phase shock wave (no fireball or detonation products).

Far-field Regime-The target is at some distance from the initiation point, greater than $10 R_{\mathrm{f}}$. In this region the blast profile is similar to the idealised blast profile (Figure 2.1), with a significant negative phase and only the shock wave to consider.

As discussed in the introduction (Chapter 1), this thesis considers the response of plates to localised, very close-in blast loading (i.e. in the detonics regime) to investigate the effect of polyurea coatings on plate deformation. As such, the dynamic pressure loading caused by the kinetic energy of the detonation products must be considered, whereas air shock effects are minimal. 


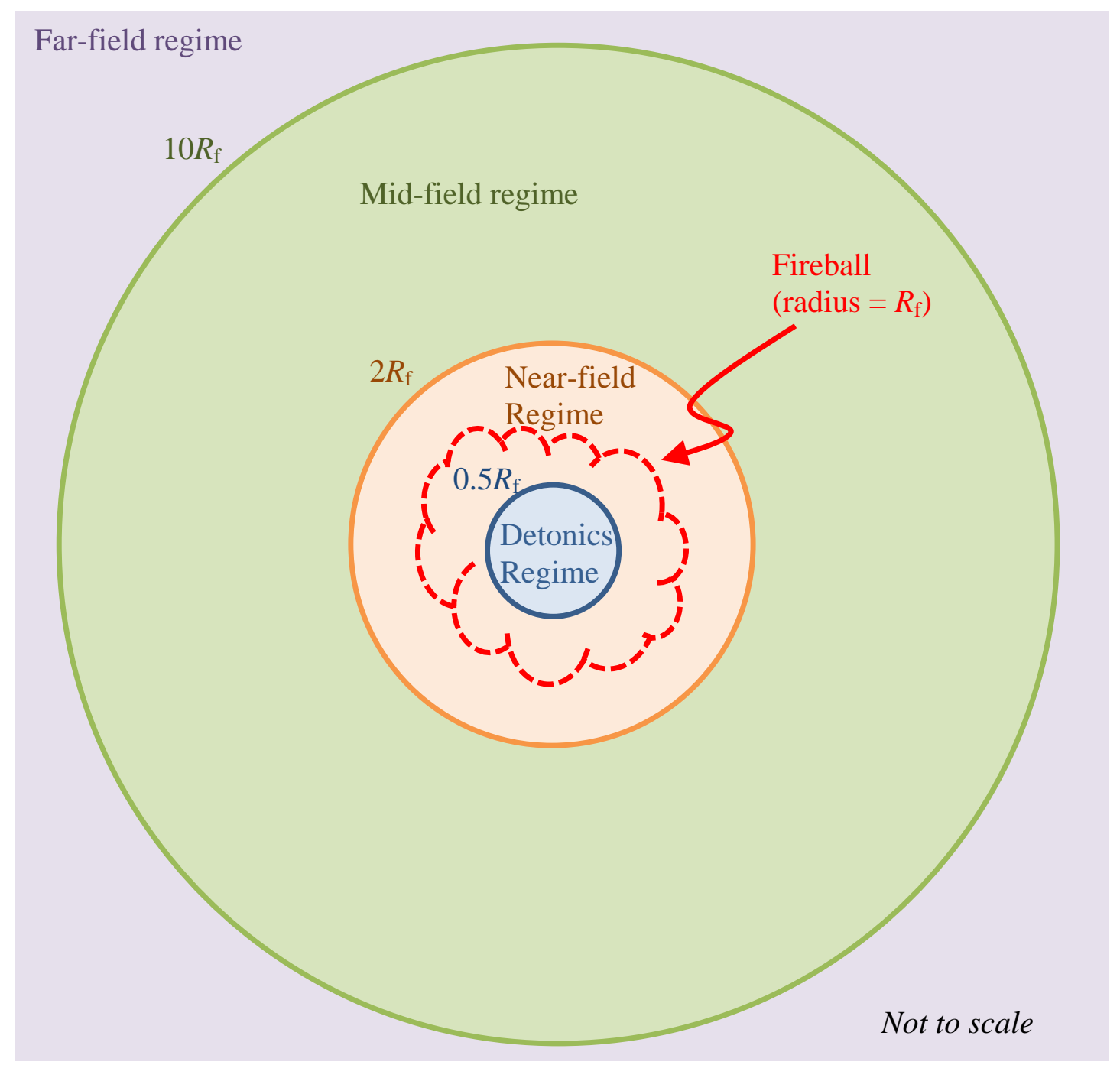

Figure 2.3 Blast regimes as defined by Ritzel (2007).

\subsection{Plate response to blast loads}

\subsubsection{Key mechanisms of blast protection}

To provide blast protection using plates, one must reduce or prevent structural failure. Menkes and Opat (1973) reported on failure modes for plates, using explosively loaded clamped beams. They reported three different modes of failure that occurred with increasing impulse. These were: Mode I-large permanent deformation, Mode II-tensile tearing at the support, and Mode III-transverse shear failure at the support. Similar failure modes have been observed for circular plates (Teeling-Smith and Nurick, 1991) and square plates (Nurick and Shave, 1996, Olsen et al., 1993). 
Wierzbicki and Nurick (1996) found that when a localized loading condition was applied by using a disc of explosive with a small radius, failure occurred by a circular area in the centre of the plate being punched out. They referred to this failure as discing. This shows that charge shape can affect plate response and failure. Other failure modes have been observed: Ramajeyathilagam (2004) observed tearing at the plate centre, and Wierzbicki (1999) reported petalling of plates under explosive loading. Petalling describes a plate that fails in a pattern representing flower petals. Figure 2.4 shows an example of petalling from a DSTO field trial.

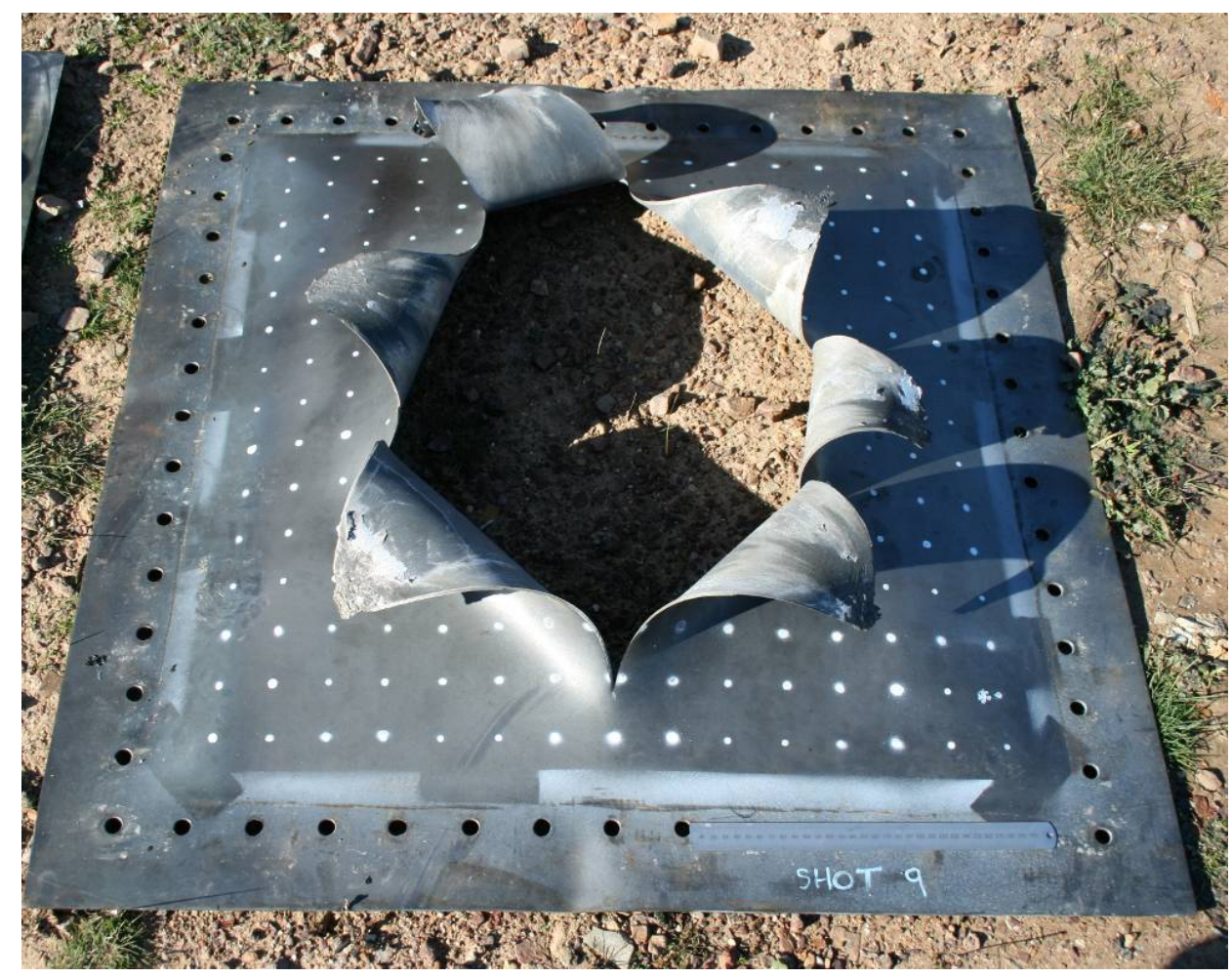

Figure 2.4 Example of plate petalling failure of a metallic plate (from a DSTO field trial).

Blast protection of a structure will generally have at least one of the following aims:

a) to reduce the permanent deformation (Mode I failure)

b) to reduce the transient elastic deformation

c) to prevent rupture (Modes II, III, discing, tearing or petalling)

Reducing the transient deformation is important in the design of blast protection systems for vehicles, and is the focus of the work in this thesis. A simplified example 
of a vehicle mine blast protection system is shown in Figure 2.5. If the deformation of the layer closest to the ground is kept low enough that it does not impact the upper layer, minimal damage will be transferred to the vehicle occupants. It is likely that plate rupture will occur beyond the deformation limits of the air gap, and as such the point of rupture is not used as a performance measure in this thesis.
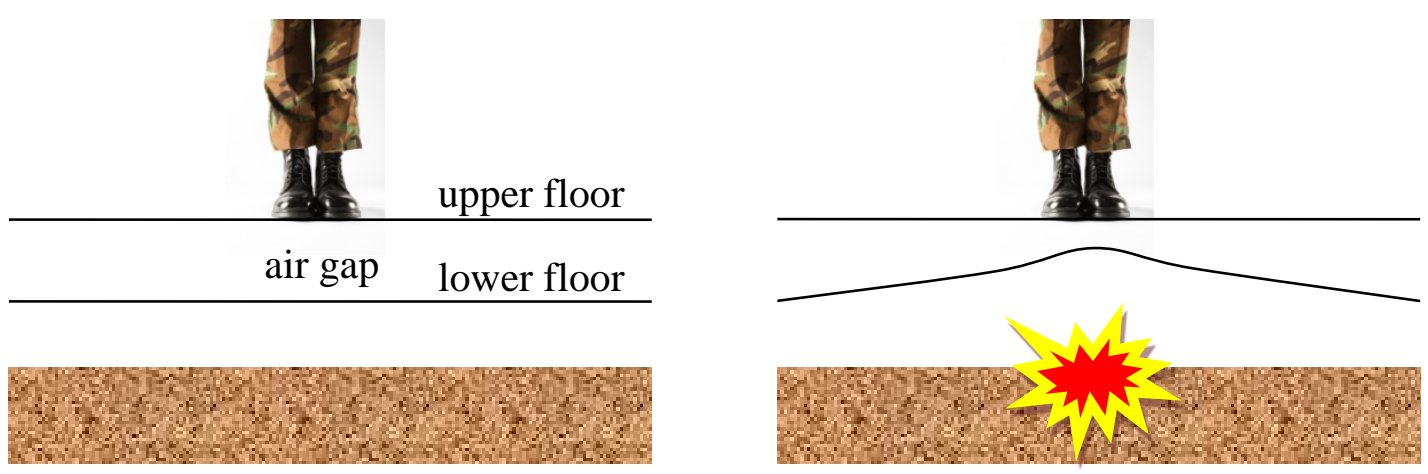

Figure 2.5 Schematic of a mine blast protection system. If the lower floor does not impact the upper floor, the occupants will incur minimal loading.

The deformation of an existing vehicle armour system may be reduced by adding extra material, and therefore mass. It is desirable to minimise the mass of a blast protection system in order to maximise payload capacity and mobility. As such, lightweight upgrades to blast protection systems are of interest. Polyurea is a material of particular interest for lightweight blast protection as it is relatively easily applied and has been shown to have favourable properties with increasing strain rate (BaheiEl-Din et al., 2006, Raman, 2011, Roland et al., 2007, Sarva et al., 2007, Yi et al., 2006). Furthermore, Sarva et al. (2007) found that polyurea undergoes a transition from a rubbery-regime behaviour at low rates to a leathery-regime behaviour at high rates. It has been suggested that the dissipation of energy during this transition may allow polyurea to provide enhanced blast protection when applied to plates, without adding significant mass (Amini, 2007). The next section provides further details about polyurea, its material properties and how it may be used to provide blast protection. 


\subsubsection{Polyurea coatings for blast mitigation}

Polyurea is a cross-linked amorphous isocyanate monomer (or prepolymer) and polyamine curative. To be classified as a polyurea, the compound must contain at least $80 \%$ polyamine (Tekalur et al., 2008). Much of the recently published work on experimental testing and characterisation of polyurea (e.g. Amini et al., 2010b, Amirkhizi et al., 2006, Qiao et al., 2011, Roland et al., 2007, Yi et al., 2006) has used a mixture of Isonate ${ }^{\circledR}$ 143L Modified MDI (formally known as 2143L) and Versalink ${ }^{\circledR}$ P1000 Oligomeric diamine.

The mechanical properties of polyurea can vary significantly depending on its stoichiometry. The manufacturer recommends a stoichiometry of 96\%, (diamine/isocyanate by mole) which corresponds to a 4:1 ratio of diamine to isocynate by weight. However, a number of researchers (Amini et al., 2010b, Amirkhizi et al., 2006, Qiao et al., 2011) have used a stoichiometry of $105 \%$ (excess isocyanate) to produce some cross-linking between polymer chains. Figure 2.6 shows the tensile stress-strain behaviour of polyurea varies significantly with stoichiometry, however toughness is reasonably constant (Roland et al., 2007). Those with higher stoichiometries will carry lower stresses and undergo larger elongations before failure.

Roland et al. (2007) also present results of tensile stress-strain measurements on polyurea at various strain rates. Figure 2.7 shows results for stress-strain curves using a drop weight apparatus, for a constant stoichiometry of $96 \%$. The yield stress and flow stress increase with increasing strain rate, whereas the failure strain decreases. 


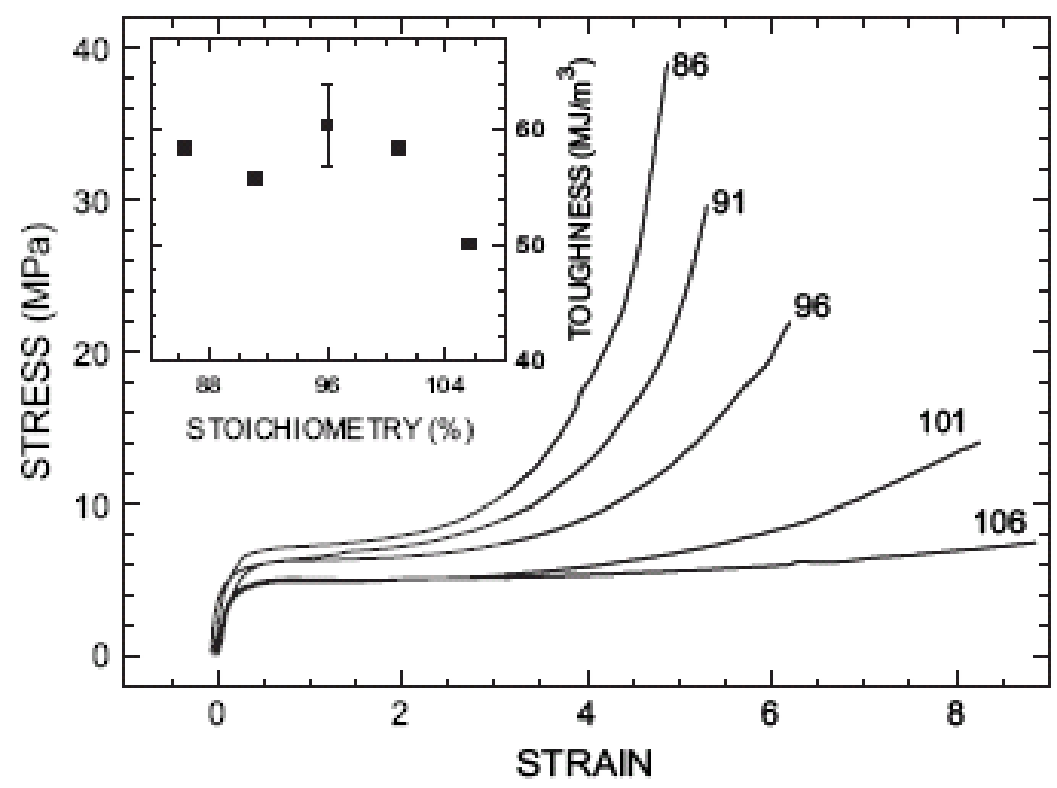

Figure 2.6 The effect of stoichiometry (86, 91, 96, 101 and 106\%) on polyurea stress-strain behaviour. The inset shows toughness as a function of stoichiometry (Roland et al., 2007).

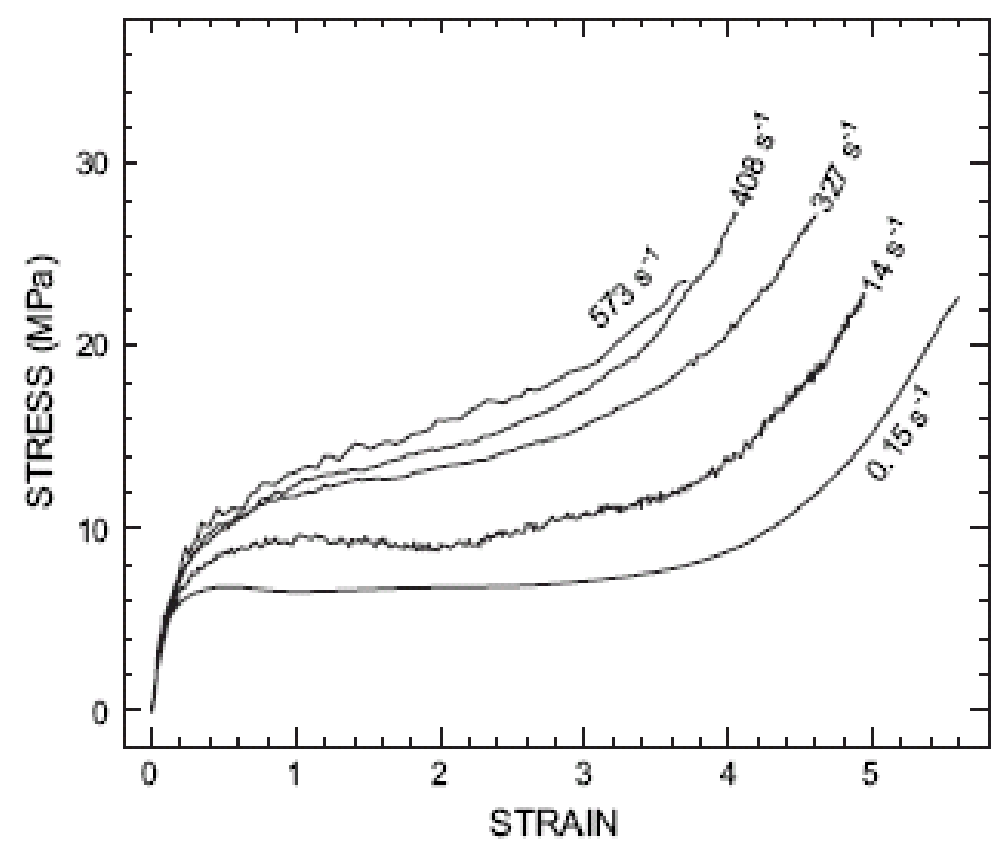

Figure 2.7 Stress-strain curves for a 96\% stoichiometry polyurea at various strain rates (Roland et al., 2007). 
The descriptions rubbery, leathery and glassy are used to describe the mechanical behaviour of polymers, which can be related to their glass transition temperature, $T_{\mathrm{g}}$. The glass transition temperature for polyurea is reported to be below $-50^{\circ} \mathrm{C}$ (e.g. Bogoslovov et al., 2007, Lee et al., 2007). At temperatures below $T_{\mathrm{g}}$, a glassy-like behaviour is observed, with the polymer exhibiting high stiffness and brittleness. Above the $T_{\mathrm{g}}$, in the rubbery regime, the stiffness is markedly lower, and the material is much more flexible. Around the $T_{\mathrm{g}}$, an intermediate "leathery" behaviour is observed. The transition between these regimes arises from the thermal energy which affects the mobility of the polymer chains at the molecular level.

Yi et al. (2006) looked at the rate-dependent compressive stress-strain behaviour of polyurea, and reported a transition from a rubbery-like behaviour at low strain rates $\left(0.001-1 \mathrm{~s}^{-1}\right)$ to a glassy-like behaviour at high strain rates $\left(\sim 10^{3} \mathrm{~s}^{-1}\right)$. Sarva et al. (2007) reported a similar behaviour, observing a transition from rubbery behaviour at the low rates $\left(\sim 0.001 \mathrm{~s}^{-1}\right)$ to leathery at high rates $\left(\sim 5000 \mathrm{~s}^{-1}\right)$. The work by Yi et al. (2006) and Sarva et al. (2007) suggest that a change in strain rate can shift the $T_{\mathrm{g}}$ of polyurea. If it can be shifted under blast loading, such that $T_{\mathrm{g}}$ is above ambient (i.e. from rubbery to a leathery or glassy regime), the increased stiffness of the polyurea may allow it to enhance its blast deformation resistance.

The method used to apply polyurea to a surface can also affect its mechanical properties. Chakkarapani et al. (2006) found significant differences between cast and sprayed polyurea. They found sprayed polyurea to have significantly higher compressibility, greater inelastic volumetric deformation, and significantly enhanced shear resistance compared to cast polyurea. An advantage of sprayed polyurea is that it can be applied in the field to pre-existing vehicle platforms and infrastructure.

Polyurea coatings have been found to enhance the blast protection of concrete wall structures (Davidson et al., 2005, Davidson et al., 2004, Johnson et al., 2005, Raman, 2011). In these applications, they are used as an add-on solution, which means they are applied to existing structures. The U.S. Air Force Research Laboratory (AFRL) performed extensive testing to explore the use polymer coatings on concrete masonry wall units (Davidson et al., 2005, Davidson et al., 2004). They initially evaluated 21 off the shelf products including polyurea, polyurethanes and polyurea/urethanes. They 
chose spray-on polyureas for further testing based on their stiffness and elongation characteristics, and their ability to enhance blast protection. When applied to the rear face of concrete masonry walls, the coatings were found to prevent spall and fragments being ejected from the rear face of the walls during the blast loading. They were also found to increase the flexural response of the walls which allowed them to deform further before they fractured (compared with an uncoated wall). In similar tests, the U.S. Army Engineer Research and Development Center (ERDC) successfully tested polymer coatings on concrete masonry wall units (Johnson et al., 2005). More recently, Raman (2011) showed that polyurea coatings can also enhance the blast protection of reinforced concrete structures.

Polyurea has also been used in the design of sandwich materials (Bahei-El-Din and Dvorak, 2007, Bahei-El-Din et al., 2006, Gardner et al., 2012), composite panels (Hui and Oskay, 2012, Kim et al., 2012, McShane et al., 2008, Tekalur et al., 2008) and as a helmet suspension pad material (Grujicic et al., 2007) to mitigate blast effects. Used in a sandwich structure, Figure 2.8, Bahei-El-Din et al. (2006) claim that the polyurea interlayer reduced facesheet strains and overall deflections due to stiffening under shock-induced compression.

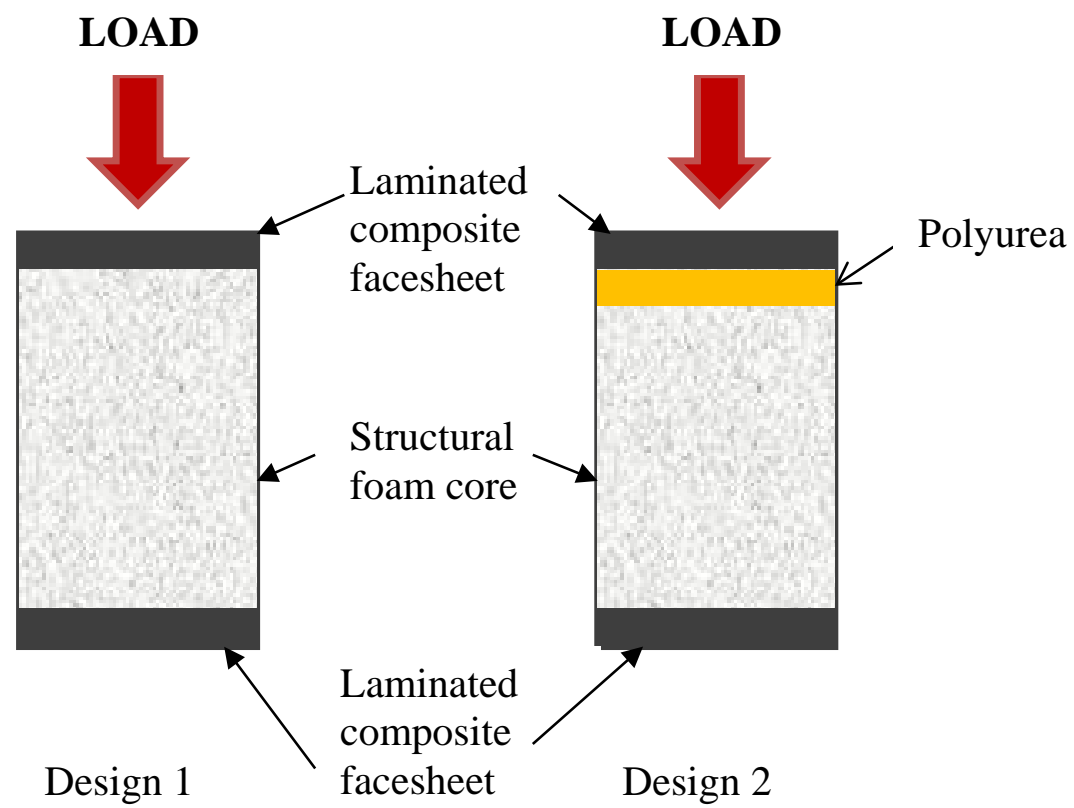

Figure 2.8 Sandwich plate designs with and without a polyurea interlayer (BaheiEl-Din and Dvorak, 2007). 
Gardner et al. (2012) conducted experimental testing on sandwich materials using a shock tube. A similar sandwich design was used to Design 2 in Figure 2.8, however the foam core used a graded design with three layers of foam of increasing density (from the loading surface to the back surface). In one configuration the polyurea layer was applied between the foam and the front facesheet (as seen in Figure 2.8, Design 2), and in the other, the polyurea was applied between the foam and the rear facesheet. They found that the latter configuration provided the best blast resistance. When the polyurea is applied in front of the rear facesheet, the stepwise compression of the core lowers the strength of the loading by the time it reaches the rear facesheet, thus reducing the overall deflection, in-plane strain, and velocity. However, when polyurea is applied behind the front facesheet and loaded in compression, its bulk stiffness is increased, thus attaining a better impedance match with the front facesheet. This has a detrimental effect in that more of the blast energy is transferred to the core. Although a graded core is effective in reducing shock wave transmission, it may not be effective for very close-in blast loading, as the loading is dominated by the detonation product expansion rather than the shock wave transmission.

Tekalur et al. (2008), also considered layered composite materials incorporating polyurea. They found experimentally that when applied to the composite E-glass vinyl ester (EVE), polyurea enhanced the blast resistance of the composite. They found that the enhanced performance was better when polyurea was applied to the front face compared to the back face, and even better when it was applied to both faces.

Hui and Oskay (2012), presented computational modelling and simulation of woven EVE composites and polyurea-coated EVE composites subjected to blast loading. Their results displayed a reasonable agreement with experimental observations reported by Tekalur et al. (2008). Predictive simulations suggested better blast mitigation characteristics can be achieved by increasing polyurea thickness. They also showed that sandwich configurations have significantly better mitigation characteristics compared to coating the outer EVE composite layers with polyurea.

Composite materials have been investigated as a base material for blast protection applications using polyurea due to their low weight. Metallic structures are also an attractive base material, particularly for vehicles, because metals generally perform 
well under ballistic and blast impacts, whilst providing structural rigidity. The concept of steel armour with a lightweight material layer is appealing as it provides structural rigidity, ballistic and blast resistance without excessive weight. However, there is little literature available on the effect of polymer coatings on the dynamic response of steel structures.

Amini et al. (2010a, 2010b) report on tests of circular steel plates subjected to impulsive loading using a reverse ballistic technique. Using this technique, a circular plate is accelerated using a gas gun and a projectile at a controlled velocity towards a polyurethane cylinder, which rests against a Hopkinson bar, Figure 2.9. The resulting impact load causes plate deformation and in some cases failure. The authors investigated the effect of applying cast polyurea to the steel plates, either on the front or back surface. They found that the polyurea applied to the loading (front) surface provided no improvement on the performance of the plate, but rather appeared to promote failure. The authors explain that this is likely due to the compression of the polyurea under the impulsive load, which increases its stiffness, thereby attaining a better impedance match with the steel plate. As a result, more energy is transferred to the plate. The application of polyurea on the rear surface of the plate reduced deformation and prevented plate fracture.

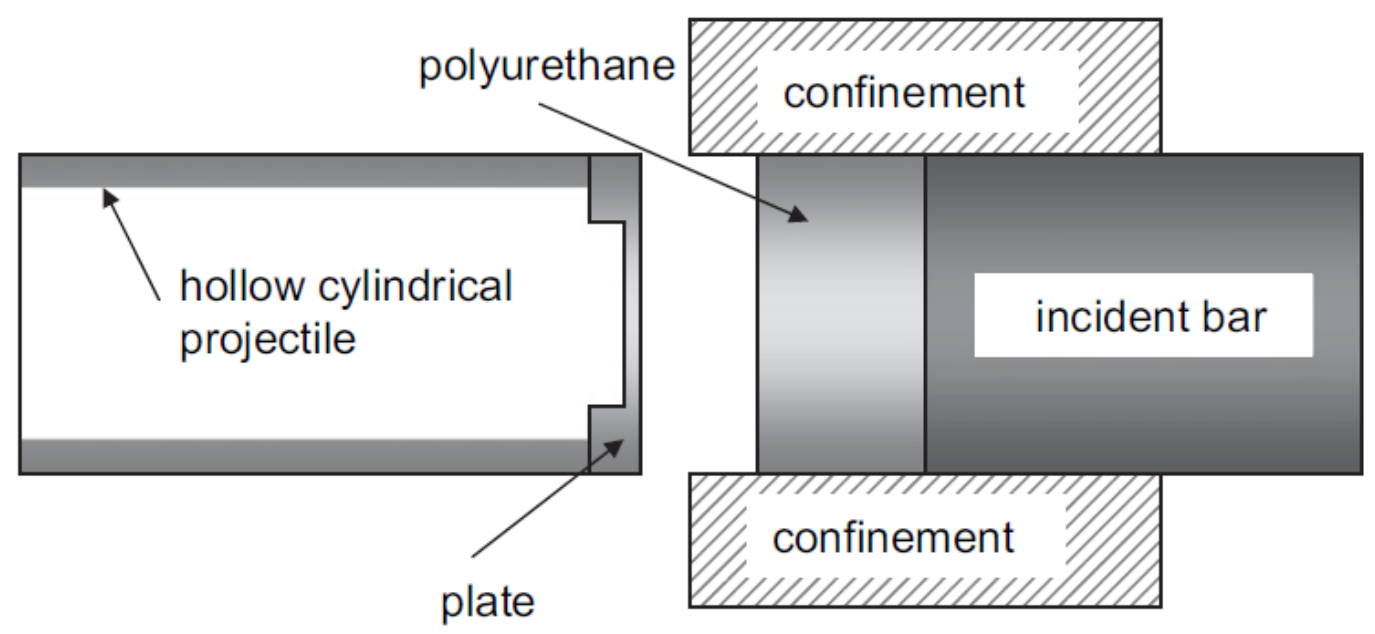

Figure 2.9 Schematic view of the reverse ballistic test experimental setup (Amini et al., 2010b). 
Previous work by DSTO showed that polyurea coatings can enhance the blast resistance of structures when applied as an appliqué (Ackland et al., 2007). In these experiments, polyurea coatings were applied to $4 \mathrm{~mm}$ thick D36 mild steel plates subjected to localised blast loads. Conventional ship steel was used, as the focus of the research was to investigate potential appliqués to increase the protection of ship hulls from terrorist blast attacks. However, the results are also applicable to military vehicles. The residual deformation of the coated plates was measured and compared to those of bare $4 \mathrm{~mm}$ mild steel plates subjected to the same loading conditions. It was found that the polymer coatings reduced the peak deformation by up to $23 \%$. Furthermore, increasing the coating thickness resulted in decreased deformation. However, the maximum reduction in deformation came at the expense of a mass increase of approximately 30\%. The results of this study are used later in this thesis for the validation of the initial numerical models, which are detailed in Chapter 3.

McShane et al. (2008) investigated the response of copper/polyurethane bilayer plates under dynamic and quasi-static loadings via static tensile, static bulge forming and dynamic bulge forming tests. Two polyurethanes were considered: PU1, which had a low tensile modulus (2.8 MPa) and a glass transition temperature of $-56^{\circ} \mathrm{C}$, and PU2, which had a much higher modulus (1120 MPa) and a glass transition temperature of $49^{\circ} \mathrm{C}$. The coatings were applied to the back faces (opposite to the loaded side) of the copper plates. The dynamic bulge forming tests were conducted using an underwater shock apparatus that consisted of a thick-walled steel tube, with the test specimen located at one end and a piston at the other. The piston was accelerated by a steel projectile fired from a gas gun, creating a shock in the water to load the test specimen. These tests indicate that on an equal mass basis, the dynamic performance of the PU2 bilayer plates with a weakly bonded polymer coating were comparable to the uncoated plates. However, when the PU2 was strongly adhered to the copper plates, their performance was inferior to that of the uncoated plates, due to cracking of the polyurethane. Thus, for this loading scenario, the authors conclude that the two polyurethane coatings do not provide dynamic performance benefits on an equal mass basis, compared to uncoated plates. The experimental study suggests that polymers with both a high modulus and strength and a high dynamic tensile ductility are required to enhance blast mitigation benefits for polymer/metal bilayers. The authors also note that the mechanical properties of polyurethane and related polyurea based 
polymers are highly tailorable by manipulation of their molecular structure, and recommend the exploration of polymers with higher modulus, strength and ductility for future work.

Although polyurea has been shown to improve blast protection by reducing the deformation of steel panels when added as an appliqué, it remains unclear whether the strain-rate hardening of polyurea is sufficient to enhance the blast protection when a polyurea-coated plate is compared with a bare steel plate of equivalent mass. As discussed previously, McShane et al. (2008) showed using underwater shock loading that the performance of a polyurethane coated copper plate is similar to that of bare copper plate of equivalent mass. However, it is unknown whether their conclusions can be applied to polyurea-coated steel plates under air-blast loading. Their suggestion for future work into tailoring of polymeric materials to improve their blast resistance was an important consideration when developing the work reported in this thesis, and is addressed in the parametric studies in Chapter 5. In order to explore the tailoring of polyurea properties to improve its blast performance, an experimentally validated numerical model of polyurea-coated steel plates is required. The next section discusses the numerical simulation techniques required to develop such a model.

\subsection{Numerical Simulation}

Numerical studies allow the prediction of the blast response of structures without the requirement for extensive experimental testing. They must however, be experimentally validated to be used with confidence. This section discusses numerical simulation methods which are used in this thesis to assess the response of polyureacoated steel plates under blast loading. Although traditional theoretical studies were not used in this thesis, they are discussed briefly in this section to give a background overview and why they were not chosen as an analytical method for this thesis. This is followed by discussions on the use of hydrocodes, methods of simulating blast/structure interaction and relevant material models. 


\subsubsection{Theoretical studies}

Many of the initial theoretical studies into the dynamic loading of plates were focused on thin circular plates with small deflections, in which only the bending effects were considered (Hudson, 1951, Richardson and Kirkwood, 1950, Taylor, 1950). These bending analyses disregarded membrane effects and, as such, are only accurate for small residual deflections which are less than about one-half the corresponding plate thickness.

As theoretical methods were developed further, membrane effects were considered and approximation techniques were used to predict large-deformation problems (e.g. Duffey (1967) and Baker (1975)). Such techniques equated the dissipated strain energy to the initial kinetic energy imparted to the circular membrane. Approximations of deformed plate profiles were assumed based on eigenvalue analyses, to find the best fit of the experimental results. This technique, known as mode approximation, was originally proposed by Martin and Symonds (1966) for small-deflection problems, however, it was later applied with success by Chon and Symonds (1977), Symonds and Chon (1979) and Nurick et al. (1987) to moderately large-deflection problems. The highest deflection-thickness ratios successfully predicted using this method were reported by Nurick et al. (1987), with deflectionthickness ratios up to 12 . Under blast loading, deflection-thickness ratios may be as high as (or higher) than 30. For such conditions, there are no theoretical models capable of accurately predicting the deformation of rectangular plates. Furthermore, bending theory, mode approximation, etc., are only applicable to Hookean perfectlyplastic materials. For hyperelastic materials such as polyurea, there are no validated models for predicting deformation under dynamic loading. Computational methods using hydrocodes overcome these limitations to allow the simulation of large deformations of polyurea-coated plates.

\subsubsection{Hydrocodes}

Hydrocodes are computer programs that can be used to numerically simulate highly dynamic events, particularly those which include shocks (Anderson, 1987). They allow complex sets of non-linear equations to be solved via the discretisation of time 
and space. Space is discretised either into a mesh of finite elements, finite differences or finite volumes, or using mesh free methods. Equations are then solved for each of the spatial elements stepping forward along discrete instants in time. Hiermaier (2007), lists some of the important characteristic elements employed in hydrocodes :

- Solutions of the conservation equations for mass, momentum and energy.

- Decoupled treatment of the stress tensor in terms of deviatoric and hydrostatic components.

- Formulation of a nonlinear equation of state accounting for shock wave formation and propagation.

- Constitutive equations for elastic and inelastic, rate-dependent material behaviour including damage, failure and post-failure behaviour.

- Arbitrary spatial and explicit time integration.

- A numerical methodology to capture shock waves.

It is the ability of hydrocodes to capture shock waves which make them ideal for the simulation of blast loading and the response of structures to blast loads. For a detailed overview of the theory of hydrocodes, Hiermaier (Hiermaier, 2007) and Zukas (Zukas, 2004) are recommended. In the following sections, some relevant concepts are discussed relating to the simulation of blast loading and structural response using hydrocodes.

\subsubsection{Simulation of blast/structure interaction}

Numerical modelling of blast loading on structures can generally be separated into two areas; modelling of the explosion and modelling of the structural response. Blast load prediction can either be calculated using empirical data or numerical methods such as computational fluid dynamics (CFD) codes. Hydrocodes such as AUTODYN ${ }^{\circledR}$ and LS-DYNA ${ }^{\circledR}$ have options to apply a time dependent load function to a surface based on empirical data from curves in TM-5-855-1 (US Army Technical Report, 1986). However, at close range this method relies on extrapolated data, and as a result is not as accurate for blast loading in the detonics regime. This method also does not account for effects such as complex shock wave reflections or large deformations of the target structure. Hence, for loading in the detonics regime and 
where large target deformations occur, the use of CFD methods for blast input is preferred.

A coupled analysis enables CFD blast simulation and the structural response calculations in the one analysis. AUTODYN and LS-DYNA are two examples of hydrocodes which allow coupled analyses. Coupling allows different numerical processors to model different components/regimes of a problem. These numerical processors each operate on separate meshes which can be coupled together in space and time to compute the interaction between structures, fluids or gases. For example, a mesh using finite volume discretisation with an Eulerian solver can be used to simulate the blast in air, whilst at the same time a mesh with a finite element discretisation and a Lagrangian solver can be used to simulate the response of a structure under the Eulerian blast load. For these types of simulations, the response of the structure as it deforms is taken into consideration in the loading calculations. This

allows the complex interaction of the blast wave and deforming structure to be simulated more accurately than using uncoupled methods. In this thesis, AUTODYN was selected as the hydrocode to be used for numerical modelling.

\subsubsection{Using finite elements to model plates}

In AUTODYN, different element types are available for the spatial discretisation of plates, namely hexahedral (hex) elements and shell elements. Both element types have advantages and disadvantages, and as such both were initially considered in this thesis in Chapter 3. This section describes the two element types, as well as their benefits and shortcomings.

The quadrilateral shell element used by AUTODYN is based on the Belytschko-Tsay element (Belytschko et al., 1984) which is derived from Mindlin plate theory. Mindilin plate elements can be regarded as special forms of solid elements, made thin in one direction (Cook et al., 1989). These elements account for both bending and transverse shear. The AUTODYN shell element has four nodes (one at each of the shell corners) and a single Gauss quadrature point located at its centre, Figure 2.10. The Gaussian integration is performed over an odd number of sublayers (the AUTODYN default is three), allowing the stress distribution through the shell to be 
defined at each of the layers. Higher orders may be used; however numerical simulations showed that the default 3rd order Gaussian integration was sufficient for this study.

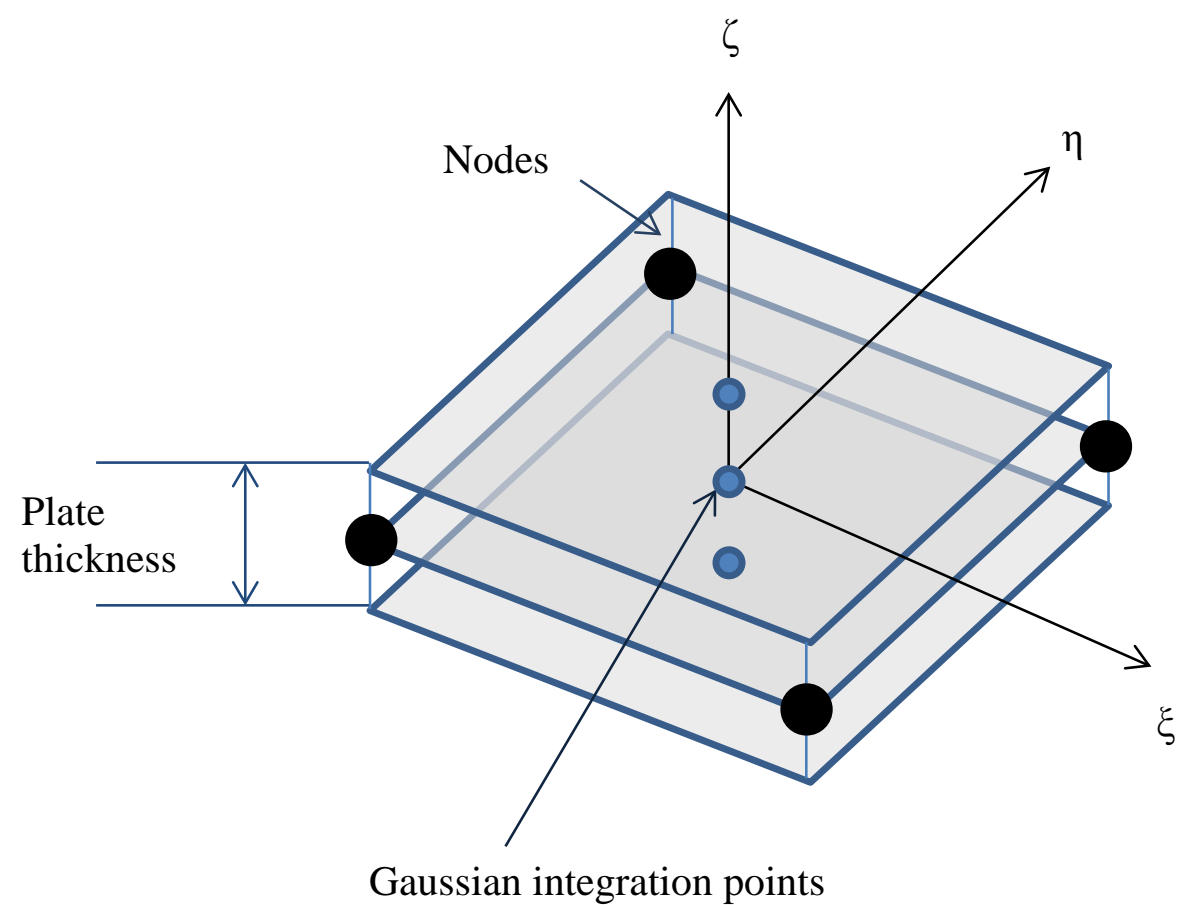

Figure 2.10 Four noded shell element with $3^{\text {rd }}$ order Gaussian integration, shown with a local coordinate system $(\xi, \eta, \zeta)$.

The hex element available in AUTODYN consists of eight nodes and has a single Gaussian integration point at its centre, Figure 2.11. 


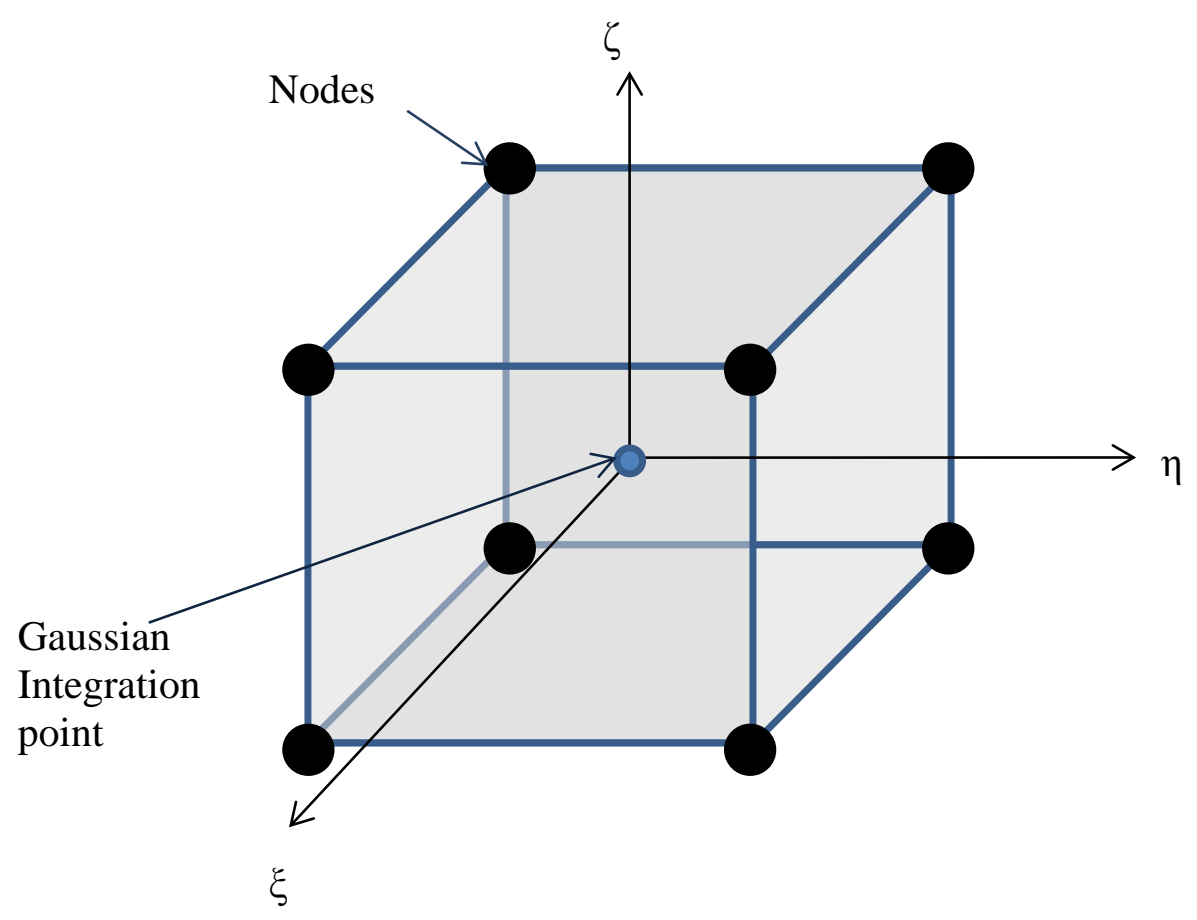

Figure 2.11 Eight noded hexahedral element with one Gaussian integration point, shown with a local coordinate system $(\xi, \eta, \zeta)$.

The main advantage of shell elements compared to hex elements is the dramatic reduction in computational time. This is achieved by the use of fewer nodes, resulting in fewer degrees of freedom, hence reducing the number of equations to be solved. Also, because the through-thickness direction of the plate is omitted in the calculation, the smallest spatial dimension is increased to the smaller edge length of the shell. This leads to much larger time steps, so fewer cycles and hence less time is required to complete the simulation. The disadvantage of using shell elements is that a number of assumptions are made in their formulation which may result in inaccurate results if used incorrectly. For example, stress wave transmission through the thickness of a shell is not possible. This may initially appear to be an issue in air-blast loading on steel plates due to the interaction of the shock wave with the plate. However, due to the large impedance difference between air and steel, the shock is almost completely reflected. The load on the plate arises from the dynamic loading from the flow of the detonation products. 


\subsubsection{Material models}

Material models are used to define the flow variables of a material by relating the stress to deformation and internal energy. In most cases, the stress tensor may be separated into a uniform hydrostatic pressure (all three normal stresses equal) and a stress deviatoric tensor associated with the resistance of the material to shear distortion (Luccioni et al., 2006). The volumetric response of a material is described by an equation of state which links the hydrostatic pressure to density (or volume) and internal energy (or temperature). A constitutive relation (or strength model) describes the deviatoric component by relating the stress in the material to the strain required to produce this stress, and may include strain rate, work hardening and thermal softening effects.

The aim of this thesis was not to develop new constitutive equations for materials, but to identify suitable existing equations to use in numerical parametric studies investigating the behaviour of polyurea-coated plates under blast loading. Descriptions of the equations of state and strength models used in this thesis are discussed in the following sections.

\subsubsection{Ideal gas equation of state}

One of the simplest forms of an equation of state is the ideal gas equation of state for an ideal polytropic gas. The form of this equation used by AUTODYN (ANSYS, 2008) is given by:

$$
p=(\gamma-1) \rho e_{\text {int }}
$$

where $p$ is the hydrostatic pressure, $\gamma$ is the adiabatic exponent, $\rho$ is the density and $e_{\text {int }}$ is the internal energy. This equation is commonly used as the equation of state for air. For low to moderate overpressure shocks (below $\sim 1.7 \mathrm{MPa}$ ), the value of $\gamma$ for air is well approximated by a value of 1.4 (Needham, 2010). However, as shock wave intensity (pressure) increases, $\gamma$ decreases, hence Equation 2.4 will lose accuracy for high overpressure shocks (>1.7 MPa). In the detonics regime of an explosion, the contribution of air (i.e. the shock wave) to the explosive loading is negligible (see Section 2.1.3); hence air does not need to be simulated in this regime. It may be 
simulated in an Eulerian volume surrounding an explosive simply to fill in the surrounding space, without affecting the explosive output.

Air is generally not modelled with a strength model as it can be considered not to carry deviatoric stress.

\subsubsection{JWL equation of state}

The detonation of explosive materials is commonly described by the Jones-WilkinsLee (JWL) equation of state (Lee et al., 1968):

$$
p=C_{1}\left(1-\frac{\omega}{r_{1} v}\right) e^{-r_{1} v}+C_{2}\left(1-\frac{\omega}{r_{2} v}\right) e^{-r_{2} v}+\frac{\omega e_{\mathrm{int}}}{v}
$$

where $v=1 / \rho$ is the specific volume, and $C_{1}, r_{1}, C_{2}, r_{2}$ and $\omega$ (adiabatic constant) are empirically determined constants. This equation is widely used because of its simplicity in hydrodynamic calculations. The constants are usually determined from cylinder expansion test data such as those described by Lee et al. (1968). These tests effectively measure the ability of the explosive material to accelerate the metal casing. Because of this, the JWL material model is considered appropriate for simulating the acceleration and deformation of steel plates where the plate is in very close proximity to the charge. Drotleff et al. (1996) advise that the model is not accurate in predicting blast pressures, and therefore is not accurate at distances where the explosive undergoes more than a two-fold expansion from its original volume. However, at contact or very close-in distances, good agreement can be found with experimental results for pressure and dynamic response. This means that the JWL equation is most suited to applications where the target is well inside the detonics regime, and the load is dominated by the detonation product expansion. For a spherical $500 \mathrm{~g}$ pentolite charge (used in this research), a two-fold volumetric expansion equates to a stand-off gap of $10.8 \mathrm{~mm}$. Although it is unclear how quickly the accuracy of the JWL equation of state deteriorates as it expands beyond this radius, it was selected as it appears to be the most accurate equation available for the detonics regime. Furthermore, it has readily available material model constants for a number of explosives which can be easily implemented into AUTODYN. 
As with air, the detonation of explosives is generally not modelled with a strength model, because when they are detonated they no longer carry deviatoric stress.

\subsubsection{Linear equation of state}

A linear equation of state assumes an isothermal process and a linear pressure-volume or pressure-density relation. This relationship generally used for fluids or solids and can be expressed by an approximation to Hooke's Law for the initial inelastic behaviour of a material as:

$$
p=\kappa \mu
$$

where $\mu=\left(\rho / \rho_{0}\right)-1$, referred to as the compaction ratio, $\rho_{0}$ is the reference density and $\kappa$ is the material bulk modulus (ANSYS, 2005). This equation of state is only suitable for fairly small compressions and should not be used if it is considered that large compressions may occur (ANSYS, 2005). For explosively loaded plates, large compressions or temperature changes are not expected as a result of the plate deformation under bending or stretching. However, a through-thickness compression wave in the plate will result in elevated localised compression. A very fine mesh through the plate thickness in addition to a more complex equation of state would be required to capture this compression wave. As the plate behaviour was not the focus of this research, the linear equation of state was considered adequate and kept constant after the initial model validation.

\subsubsection{Johnson - Cook strength model}

The Johnson-Cook strength model defines the flow stress in terms of strain, strain rate and temperature. It is valid for materials subjected to large strains, high strain rates and high temperatures, and is defined as (Johnson and Cook, 1983):

$$
\bar{\sigma}=\left[\sigma_{0}+B \bar{\varepsilon}_{p}^{n}\left[1+C \log \left(\frac{\dot{\bar{\varepsilon}}_{p}}{\dot{\varepsilon}_{0}}\right)\right]\left[1-\hat{\theta}^{m}\right]\right.
$$

where $\quad \hat{\theta}=\frac{T-T_{r}}{T_{\text {melt }}-T_{r}}$ 


$$
\begin{aligned}
& \sigma_{0}=\text { yield stress at low strains }(\mathrm{Pa}) \\
& \bar{\sigma}=\text { yield stress at non-zero strain rate }(\mathrm{Pa}) \\
& \bar{\varepsilon}_{p}=\text { equivalent plastic strain } \\
& \dot{\bar{\varepsilon}}_{p}=\text { normalized equivalent plastic strain rate }\left(\mathrm{s}^{-1}\right) \\
& \dot{\varepsilon}_{0}=\text { reference strain rate }\left(\mathrm{s}^{-1}\right) \\
& T=\text { material temperature }(\mathrm{K}) \\
& T_{r}=\text { room temperature }(\mathrm{K}) \\
& T_{m e l t}=\text { material melting temperature }(\mathrm{K})
\end{aligned}
$$

$B, n, C$ and $m$ are material dependent parameters that may be determined empirically. The yield strength of the material, $\sigma_{0}$, along with parameters $B$ and $n$ can be derived from quasi-static tension tests. The strain rate parameter $C$ is identified through dynamic tension tests at varying strain rates. Since temperature influences in the course of dynamic characterization tests are hard to separate from the rate effects, the temperature influence represented through the third term in the Johnson-Cook model is often omitted (Hiermaier, 2007). Johnson-Cook strength model parameters for many materials are available in the AUTODYN material library and literature.

\subsubsection{Constitutive models for polyurea}

Several researchers have published constitutive models for polyurea. Amirkhizi et al. (2006) describe an experimentally-based viscoelastic constitutive model for polyurea, including pressure and temperature effects. They present high strain-rate data obtained using Hopkinson bar experiments, the results of which suggest that the strength of polyurea is highly pressure dependent. Chakkarapani et al. (2006) report on the multiaxial compressive behaviour of polyurea. They found that the bulk deformation of cast polyurea was non-linear and nearly reversible, and could be modelled by Tait's equation for the compressibility of seawater. They also found that the material's shear resistance is very low and could be ignored for practical purposes. Li and Lua (2009) 
present a constitutive model for polyurea that includes both hyperelastic and viscoelastic behaviour. The Ogden model (Ogden, 1972) is used for the hyperelastic part and its parameters are curve fitted using quasi-static test data. A nonlinear viscoelastic model is employed for describing the viscoelastic behaviour and its relaxation time is obtained based on shear relaxation modulus test data. More recently, Shim and Mohr (2011) conducted experiments at different strain rates to characterise the large-deformation behaviour of polyurea under compressive loading. These experiments included continuous loading and unloading experiments and uniaxial compression tests. Based on the results, they present a new constitutive model for polyurea.

The Mooney-Rivlin model defines the strain energy function, $\psi$, of a hyperelastic material as (from (ANSYS, 2008)):

$$
\psi=\sum_{p, q=0}^{N} C_{p q}\left(\bar{I}_{1}-3\right)^{p}\left(\bar{I}_{2}-3\right)^{q}+\frac{1}{d}(J-1)^{2}
$$

where $C_{p q}$ are empirically determined material constants, $\bar{I}_{1}$ and $\bar{I}_{2}$ are the first and the second invariant of the deviatoric component of the left Cauchy-Green deformation tensor, $J$ is the determinant of the elastic deformation gradient, and $d$ is the material incompressibility parameter. The expansion series in Equation 2.8 forms the deviatoric component, where the constants can be fit to material stress-strain data. The second term is the volumetric component, where $d$ is equal to $2 / \kappa$, and $\kappa$ is the bulk modulus. $\bar{I}_{1}$ and $\bar{I}_{2}$ are defined as (Charlton et al., 1994):

$$
\begin{aligned}
& \bar{I}_{1}=\lambda_{1}^{2}+\lambda_{2}^{2}+\lambda_{3}^{2} \\
& \bar{I}_{2}=\left(\lambda_{1} \lambda_{2}\right)^{2}+\left(\lambda_{2} \lambda_{3}\right)^{2}+\left(\lambda_{3} \lambda_{1}\right)^{2}
\end{aligned}
$$

where $\lambda$ is known as the "stretch ratio", defined as the ratio of the current to the original length. Subscripts 1 to 3 denote three mutually orthogonal directions. In the case of uniaxial tension, $\lambda_{2}=\lambda_{3}=\lambda_{1}{ }^{-1 / 2}$, giving: 


$$
\begin{aligned}
& \bar{I}_{1}=\lambda^{2}+2 \lambda^{-1} \\
& \bar{I}_{2}=2 \lambda+\lambda^{-2}
\end{aligned}
$$

The uniaxial engineering tensile stress can be found using the equation:

$$
\sigma=2\left(\lambda-\frac{1}{\lambda^{2}}\right)\left(\frac{\partial \psi}{\partial \bar{I}_{1}}+\frac{1}{\lambda} \frac{\partial \psi}{\partial \bar{I}_{2}}\right)
$$

This equation can be used to determine the Mooney-Rivlin constants from a uniaxial tensile stress-strain curve.

For the deviatoric response (i.e. the expansion series in Eq. 2.8), 2, 3, 5 and 9 parameter fits are generally used. Mac Donald (2007) states that as a general rule, the number of parameters should be chosen depending on the number of inflections seen in the stress-strain curve. Typical curves are shown in Figure 2.12. If a single curvature is present (no inflections), a 2-parameter model is sufficient. A curve with a single inflection can be captured by a 5-parameter model, and a curve with two inflections can be captured by a 9-parameter model.
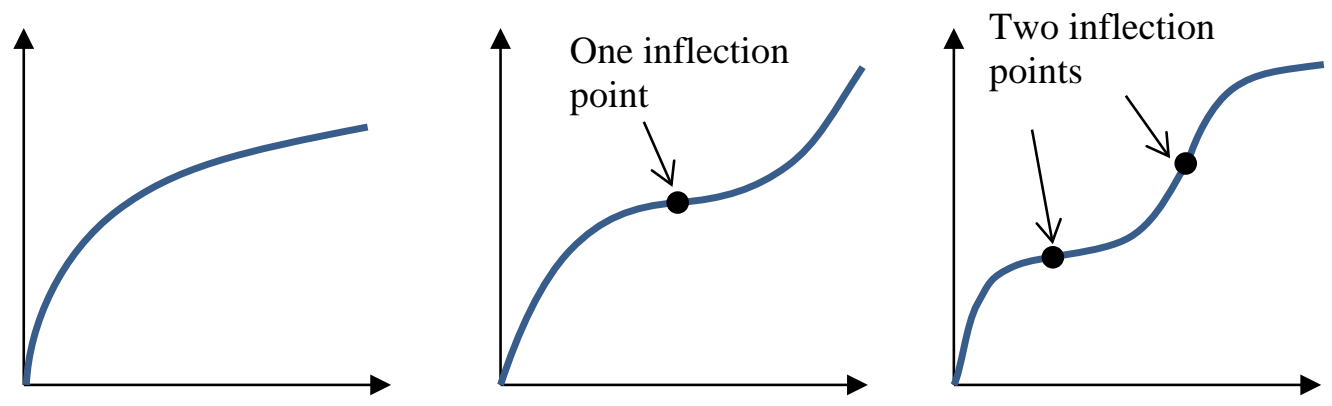

Figure 2.12 Typical shapes of Mooney-Rivlin stress-strain curves.

A 2-parameter version of this model was used by Xue and Hutchinson (2007) to model polyurea and its effect on necking in bilayer plates under impulsive loads. More recently, Xue et al. (2010) used a 6-parameter Mooney-Rivlin model to investigate the effects of polyurea coatings on ballistic performance of DH-36 steel 
plates. Referring to Figure 2.7 in Section 2.2.2, the stress-strain curves for polyurea reported by Roland et al. (2007) appear to have one inflection. However, for strains of up to approximately three, the curve has not yet inflected, and as such, a 2-parameter model can be used. The 2-parameter model is derived from Equation 2.8 by setting $N$ $=1$ and $C_{00}=C_{11}=0$, to give:

$$
\psi=C_{10}\left(\bar{I}_{1}-3\right)+C_{01}\left(\bar{I}_{2}-3\right)^{q}+\frac{1}{d}(J-1)^{2}
$$

Finney and Kumar (1987) describe how the constants $C_{01}$ and $C_{10}$ can be fit to a 2-parameter Mooney-Rivlin material stress-strain curve. For the special case of uniaxial tension of an incompressible Mooney-Rivlin material, the stress-strain equation can be derived from Equations 2.10 to 2.12 and expressed as a 2-parameter model:

$$
\sigma=2\left(\lambda-\lambda^{-2}\right)\left(C_{10}+C_{01} \lambda^{-1}\right)
$$

where $\sigma$ is the Cauchy or engineering stress and $\lambda$ is the stretch ratio. For a MooneyRivlin hyperelastic material, plotting $\sigma /\left[2\left(\lambda-\lambda^{-2}\right)\right]$ against $1 / \lambda$ yields a straight line. The slope of the line is equal to $C_{01}$ and the intercept at $1 / \lambda=1$ is $C_{01}+C_{10}$. For compressible and incompressible materials, the initial shear modulus, $G$, is related to the material constants by $G=2\left(C_{10}+C_{01}\right)$. The initial tensile modulus, $E$, is given by $E=6\left(C_{10}+C_{01}\right)$ for an incompressible material and $E=(9 \kappa G) /(3 \kappa+G)$.

\subsection{Summary}

Although there exist some preliminary studies which investigate the effect of polyurea coatings on steel structures under impulsive loading (Ackland et al., 2007, Amini et al., 2010a, Amini et al., 2006, Amini et al., 2010b, Chen et al., 2008), the mechanisms by which they work are not fully understood. It is unclear whether polyurea-coated plates perform better under explosive blast loading compared to bare steel plates of the same mass. It is also unclear how different polyurea properties affect its blast performance, and how such coatings might be improved. 


\section{Chapter 3}

\section{Preliminary Experiments and Numerical Validation}

\subsection{Introduction}

This chapter presents the initial experimental and numerical studies of polyurea-coated plates under blast loading. In the literature review (Chapter 2), previous blast testing by DSTO on polyurea-coated plates (Ackland et al., 2007) was discussed. These experiments did not include any measurement of the transient deformation of the plates. The experimental blast testing of bare steel plates presented in this chapter was conducted to refine the experimental technique, including a method for measuring the transient response. The experimental results, both on bare steel plates and those with polyurea coatings, also are used for the validation of numerical models. The development of the numerical models is described in detail in this chapter, including mesh discretisation, mesh resolution studies, material model selection and issues encountered when using different element types to model the plates. The experimental methods and numerical models developed in this chapter form the basis of the research presented in the rest of this thesis.

\subsection{Description of the validation experiments}

In Ackland et al. (2007), steel plates were tested against localised blast loading with polyurea coatings applied to the back surface of some of the plates. Tests were conducted at the Department of Defence's Proof \& Experimental Establishment in Graytown, Victoria. Figure 3.1 shows the experimental setup. The blast tests were conducted using $500 \mathrm{~g}$ spherical charges of pentolite, which were suspended centrally in front of the steel plate. For each event the charge was detonated using RP501 exploding bridgewire (EBW) detonators located centrally within the charge. 


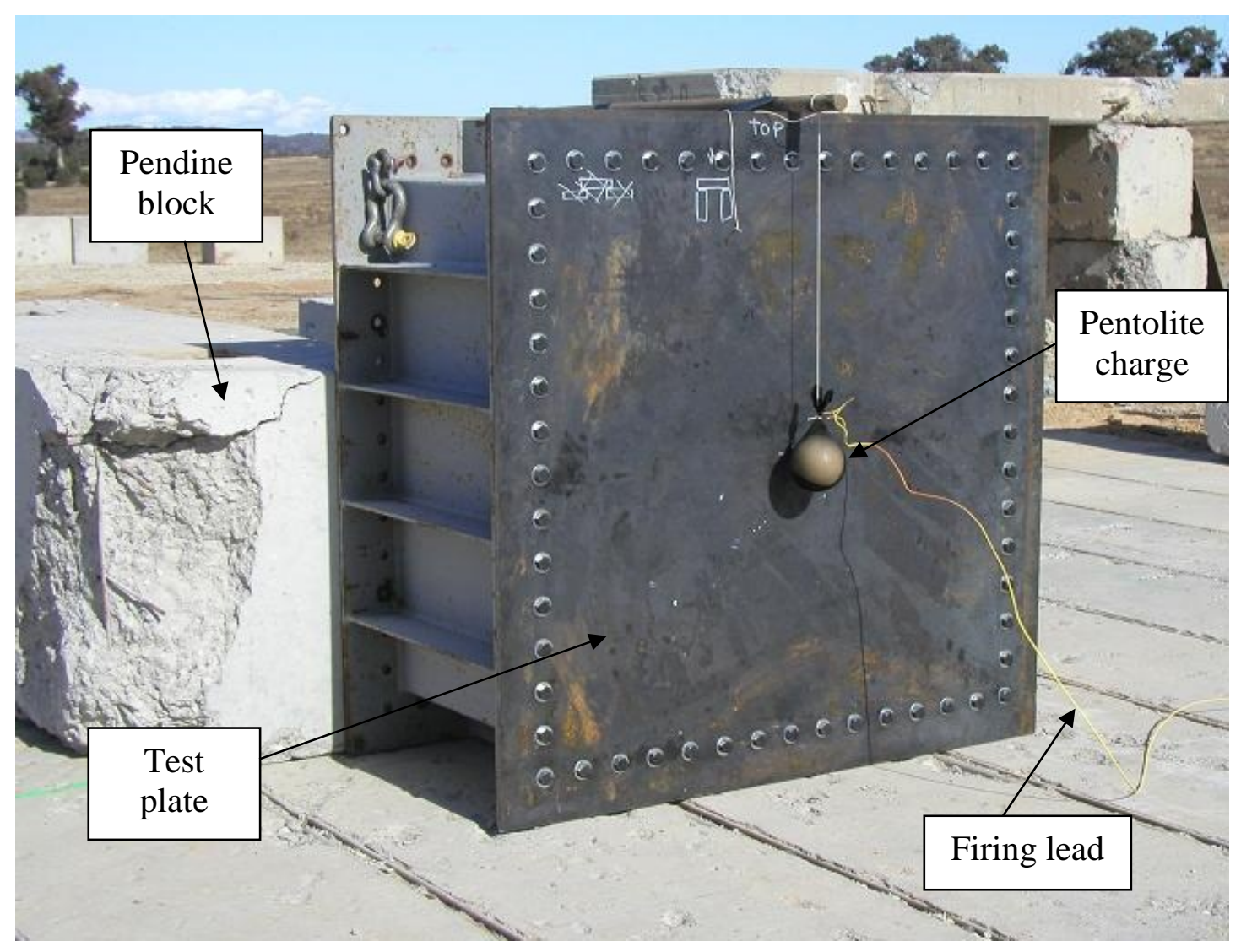

Figure 3.1 Charge and plate setup of the validation experiments from Ackland et al. (2007).

The test plate was bolted to a test rig and two pendine blocks were positioned behind the rig to restrict its movement. For the plates with the polyurea coatings, the steel was positioned with the coating on the opposite side to the pentolite charge. Four pressure gauges were set up at ground level; two at $5 \mathrm{~m}$ and two at $10 \mathrm{~m}$ from the charge, at ground level. The gauges were positioned to verify the consistency of the blast wave output from each device, e.g. an incomplete detonation would be seen as an inconsistency in the pressure gauge data. The gauges are not used to estimate the load on the plate surface, as they measure the mid-field static pressure, whereas the load is dominated by the dynamic pressure from the expanding detonation products.

Test panels of $1000 \mathrm{~mm} \times 1000 \mathrm{~mm}$ were used for each experiment, with a test area of $820 \mathrm{~mm} \times 820 \mathrm{~mm}$ when mounted on the blast rig. The maximum plastic deformation of the plates was measured as the deformation of the front (blast-side) surface of the plate at the plate centre, while the plate was still attached to the rig, 
Figure 3.2. This was achieved by placing a level ruler across the front of the plate and lowering a vernier caliper into the bulge at its deepest point.
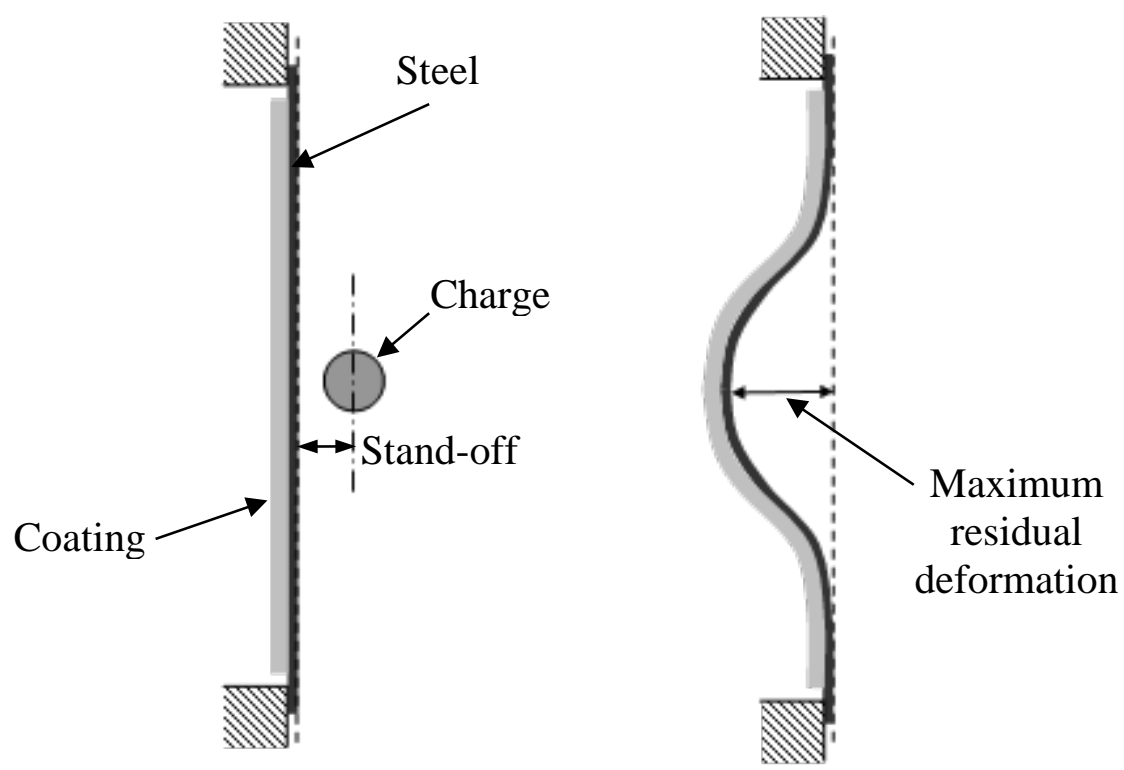

Figure 3.2 Flat specimen pre-test (left); deformed specimen after testing (right). The stand-off was varied between the experiments.

Bare plates of $4 \mathrm{~mm}$ D36 steel (a conventional ship steel) were tested as a baseline for comparison with polyurea-coated plates. Two plates were coated on their back face with an in-house formulation comprising a blend of a polyether prepolymer VPLS 2371 and DT Hardener (both from Bayer). The coating was cast directly onto the steel plates which had been previously grit blasted and primed. The coating was not applied in the region where the plates were bolted to the support frame, since it would have interfered with the rigid-like boundary conditions.

Additional explosive testing was performed in July 2007 at the Department of Defence’s Proof \& Experimental Establishment in Graytown, Victoria, against bare $4 \mathrm{~mm}$ thick Bluescope XLERPLATE 350 steel plates. This steel was chosen due to its price and availability, and because material models for mild steels are readily available in literature and numerical modelling programs. Its properties, along with those for D36, are shown in Table 3.1. As with the previous experiments, test plates were $1000 \mathrm{~mm} \times 1000 \mathrm{~mm}$ with a test area of $820 \mathrm{~mm} \times 820 \mathrm{~mm}$ when mounted on the blast rig. Two identical tests were conducted to verify the consistency of the 
results. The charges used were $500 \mathrm{~g}$ pentolite spheres, which were centrally detonated and positioned with their centre of mass $77.5 \mathrm{~mm}$ above the plate surface, as shown in Figure 3.3. The rig orientation was changed such that the test plate was horizontal instead of vertical. In the previous tests, the test rig was displaced significantly by the blast, landing in a horizontal position. The new arrangement was used to minimise movement of the test rig under the blast load. In both series of tests, the charges were suspended in a stocking from a wooden frame to ensure minimal interference with the formation of the blast wave.

Table 3.1 Test plate mechanical properties (Bluescope Steel, 2009, MIL-S22698C, 1988).

\begin{tabular}{lllll} 
& \multicolumn{2}{l}{ Yield Strength (MPa) } & \multicolumn{2}{l}{ Tensile Strength (MPa) } \\
\cline { 2 - 5 } & $\begin{array}{l}\text { Guaranteed } \\
\text { minimum }\end{array}$ & Typical & $\begin{array}{l}\text { Guaranteed } \\
\text { minimum }\end{array}$ & Typical \\
\hline D36 & 355 & N/A & N/A & $490-620$ \\
$\begin{array}{l}\text { Bluescope 350 } \\
\text { XLERPLATE }\end{array}$ & 360 & $410-540$ & 450 & $480-590$ \\
\hline
\end{tabular}




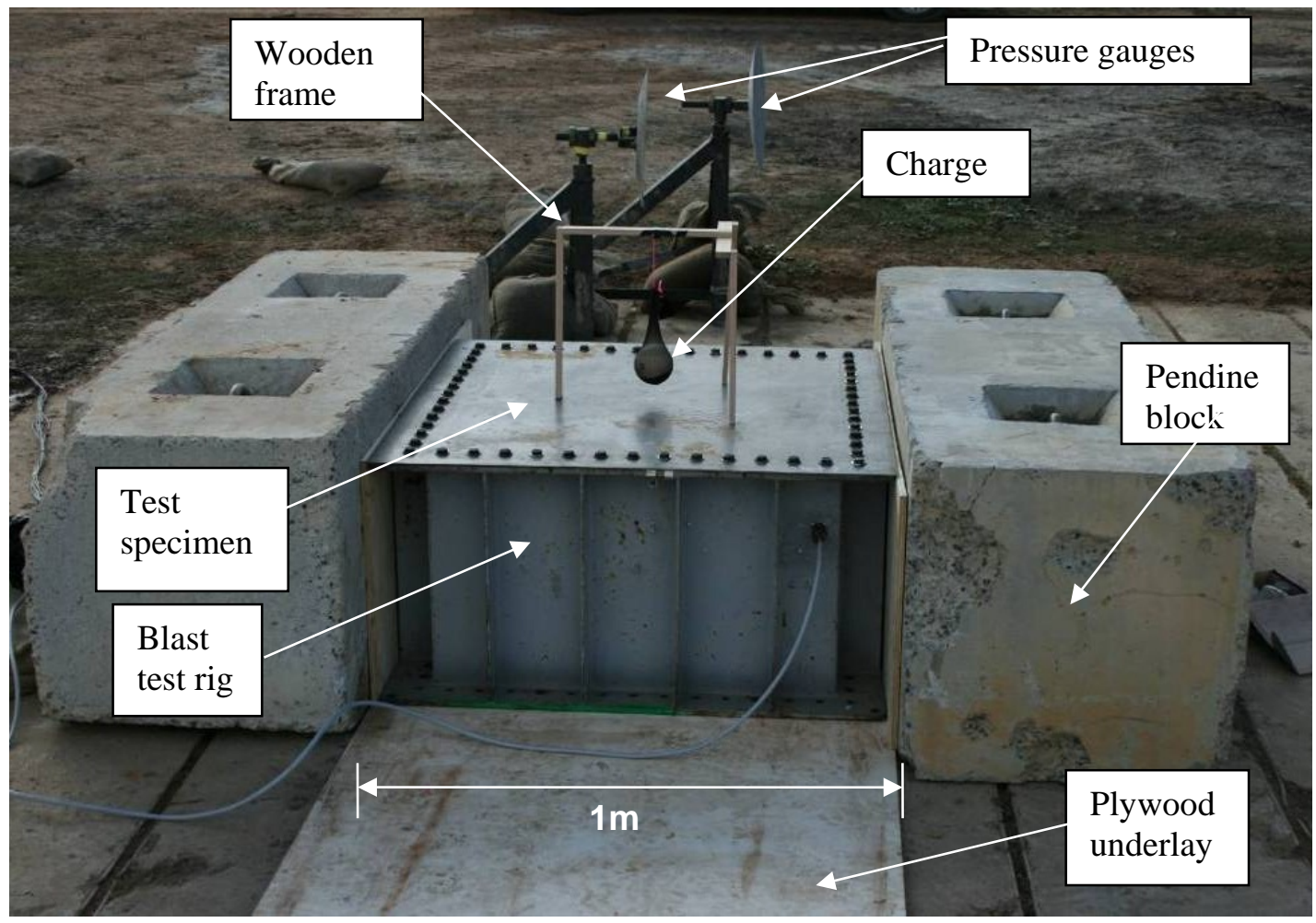

Figure 3.3 Experimental setup for the July 2007 Experiments.

An array of 12 wires was set up underneath the test plate to measure the transient deformation of the plate. The top wire was positioned $50 \mathrm{~mm}$ from the back surface of the plate and the subsequent wires were placed at $11.6 \mathrm{~mm}$ intervals. As the plate deformed and made contact with each of the wires a circuit was completed and the time of contact was recorded by the data acquisition unit as a drop in voltage, which was sampled at 5.0 MHz. From these measurements, discrete data points were plotted to generate a deformation-time history for each test. Figure 3.4 shows a schematic of the electrical circuit and Figure 3.5 shows the wire array in the test rig before the top plate was bolted to the rig. 
Chapter 3. Preliminary Experiments and Numerical Validation.

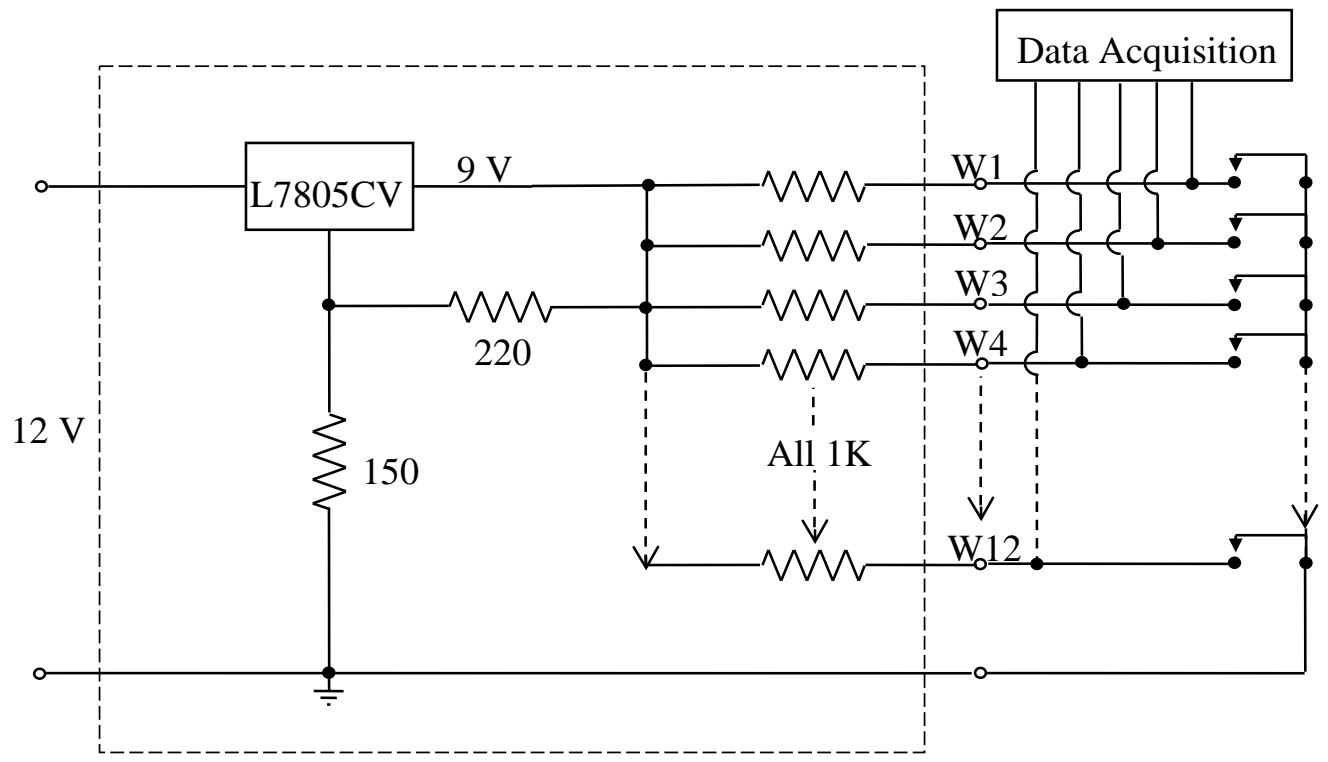

Figure 3.4 Electrical schematic for the measurement of the plate transient deformation. The switches represent the plate (which was grounded) contacting each wire, $\mathrm{W}_{\mathrm{n}}$, in turn during its deformation stage, dropping the potential to approximately zero.

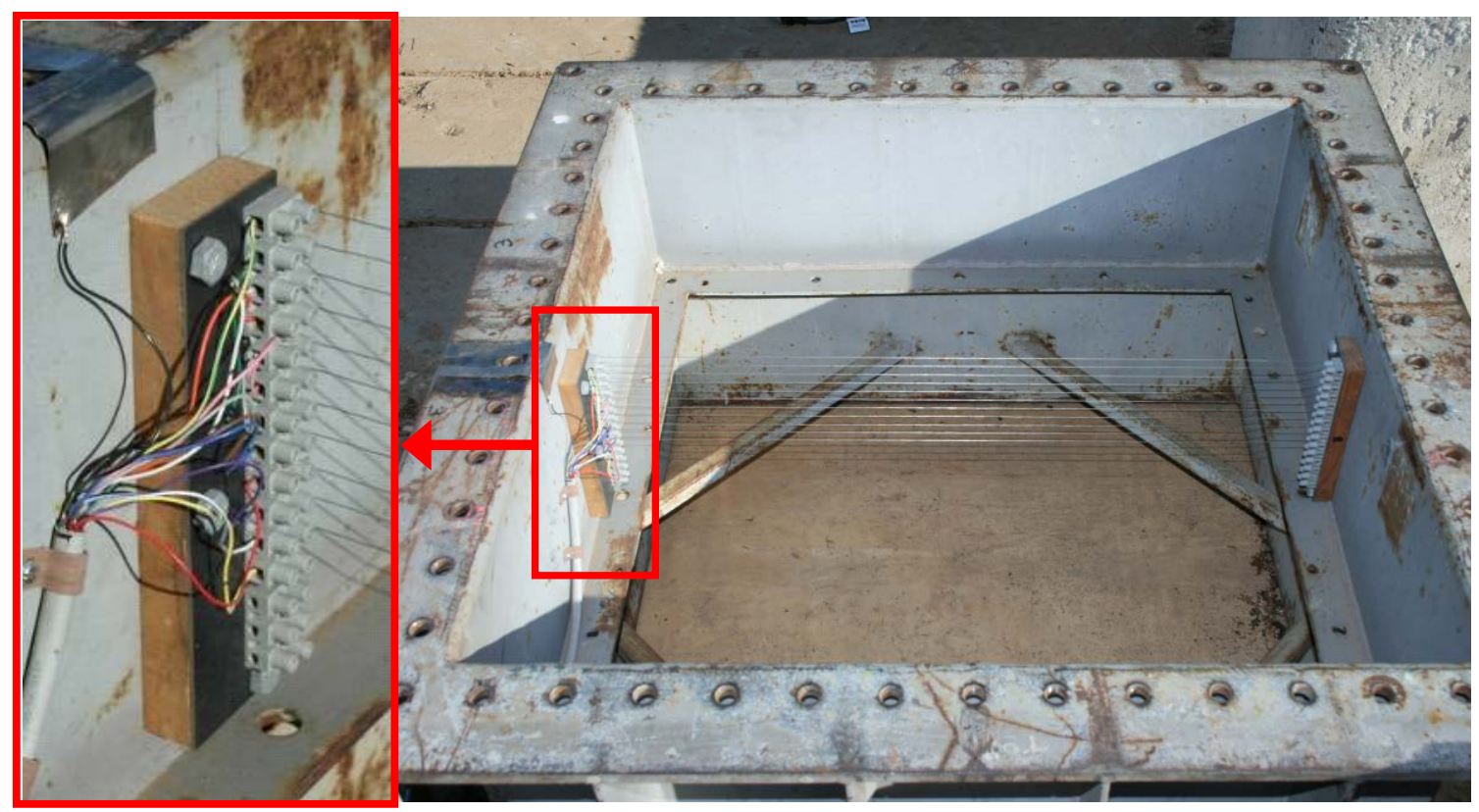

Figure 3.5 Wire deformation measurement array as set up before the blast event. 


\subsubsection{Validation experiment results}

A summary of the validation experiments and results is given in Table 3.2. It is noted that the stand-off varies throughout the tests. Plates V1 and V2 were used in a separate experiment in which the stand-off was selected to give a reasonable amount of deformation without approaching plate rupture. Plates V3 and V4 were used to tune the standoff to achieve a maximum deformation without plate rupture. Plates V5 and V6 had a slightly larger standoff than V3. The exact reason is not known as these experiments were conducted before this research was commenced; however it is believed to be due to an experimental variation.

Table 3.2 Results of the validation experiments.

\begin{tabular}{llllll}
\hline Test & Backing Steel & $\begin{array}{l}\text { Polyurea } \\
\text { coating } \\
\text { thickness } \\
(\mathrm{mm})\end{array}$ & $\begin{array}{l}\text { Polyurea coating } \\
\text { areal density } \\
\left(\mathrm{kg} / \mathrm{m}^{2}\right)\end{array}$ & $\begin{array}{l}\text { Charge } \\
\text { stand-off } \\
(\mathrm{mm})\end{array}$ & $\begin{array}{l}\text { Maximum } \\
\text { residual } \\
\text { deformation } \\
(\mathrm{mm})\end{array}$ \\
\hline V1 & $4 \mathrm{~mm}$ mild steel & None & N/A & 77.5 & 120 \\
V2 & $4 \mathrm{~mm}$ mild steel & None & N/A & 77.5 & 120 \\
V3 & $4 \mathrm{~mm}$ D36 & None & N/A & 60.5 & 143 \\
V4 & $4 \mathrm{~mm}$ D36 & None & N/A & 56 & Rupture \\
V5 & $4 \mathrm{~mm}$ D36 & $10 \mathrm{~mm}$ & 9.3 & 61.5 & 134 \\
V6 & $4 \mathrm{~mm}$ D36 & $19 \mathrm{~mm}$ & 18.9 & 61.5 & 109 \\
\hline
\end{tabular}

${ }^{*}$ Tearing at plate centre.

Figure 3.6 shows an example of polyurea-coated plate after being removed from the blast rig. For the polyurea-coated plates, evidence of de-bonding was seen across a circular area at the centre of the plate. The pressure gauge measurements were consistent throughout the tests, indicating a reliable detonation for each of the events. 


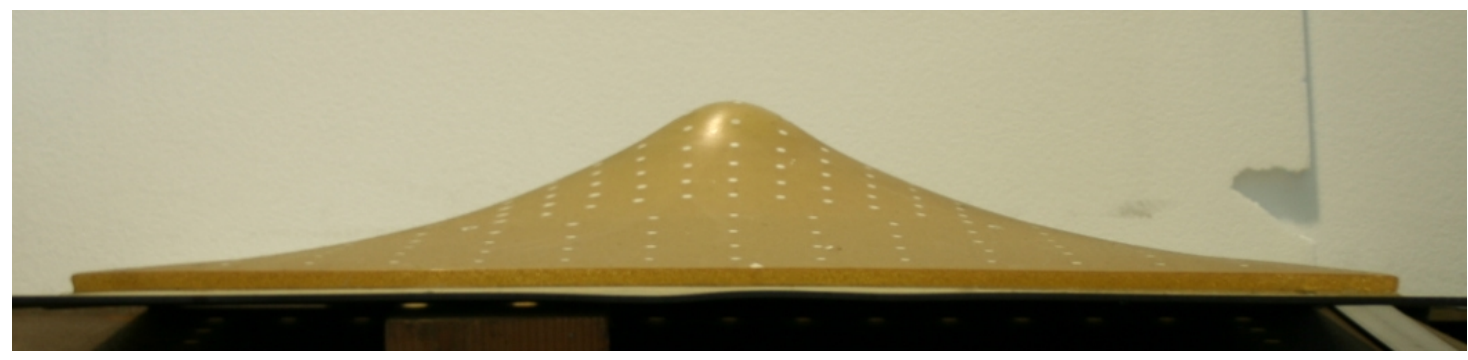

Figure 3.6 Example of a coated plate after the experiment (test V5).

Figure 3.7 and Figure 3.8 show the results recorded by the deformation wires for the tests $\mathrm{V} 1$ and $\mathrm{V} 2$, together with the post-test residual deformation measurement for test V1. The data suggests a peak transient deformation of at least $131 \mathrm{~mm}$ but no more than $143 \mathrm{~mm}$ for both plates, as the wire at $131 \mathrm{~mm}$ made contact with the plate however the wire at $143 \mathrm{~mm}$ did not contact the plate. For test V2, the plate arrived at the $131 \mathrm{~mm}$ wire slightly earlier compared to test V1. This may indicate that the plate in test V2 was travelling slightly faster than in test V1; however it may also be due to a slight variation in the wire positions as they were tensioned between the two events. A more accurate method to capture the transient deformation would be the use of high speed photography. Although this was not available during these preliminary trials, high speed photography is used in the explosive experiments presented in Chapter 4. 
Chapter 3. Preliminary Experiments and Numerical Validation.

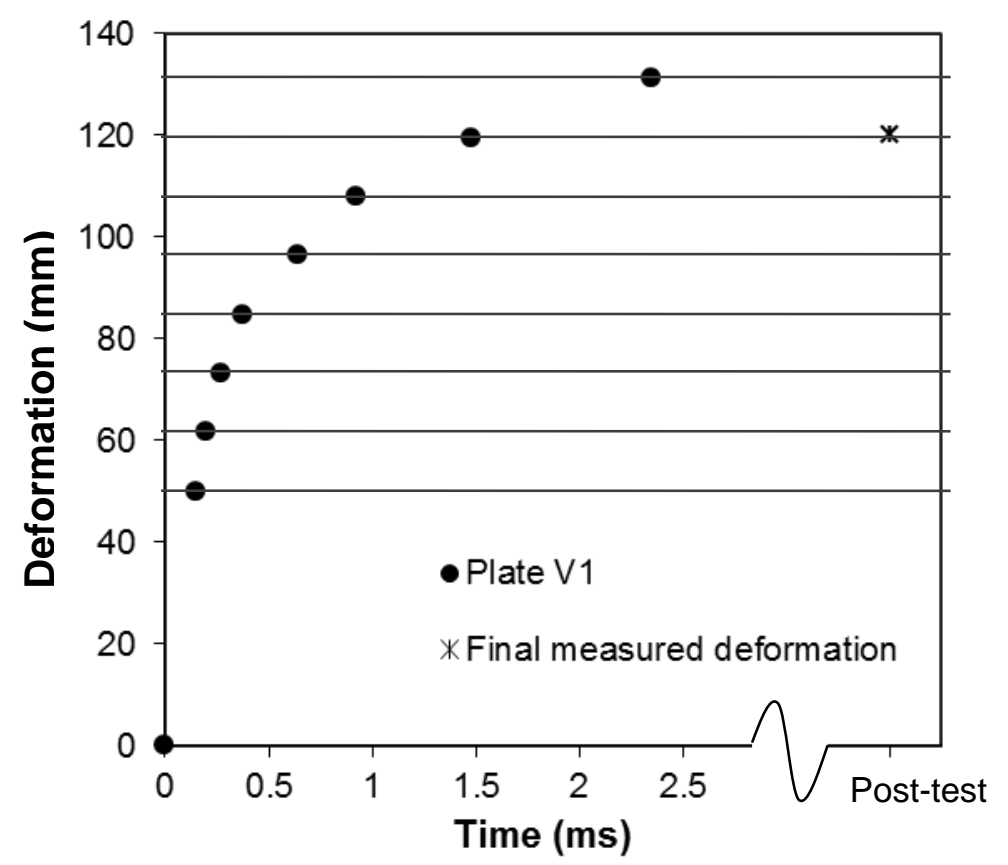

Figure 3.7 Deformation wire results for test V1. The horizontal lines represent the wire locations.

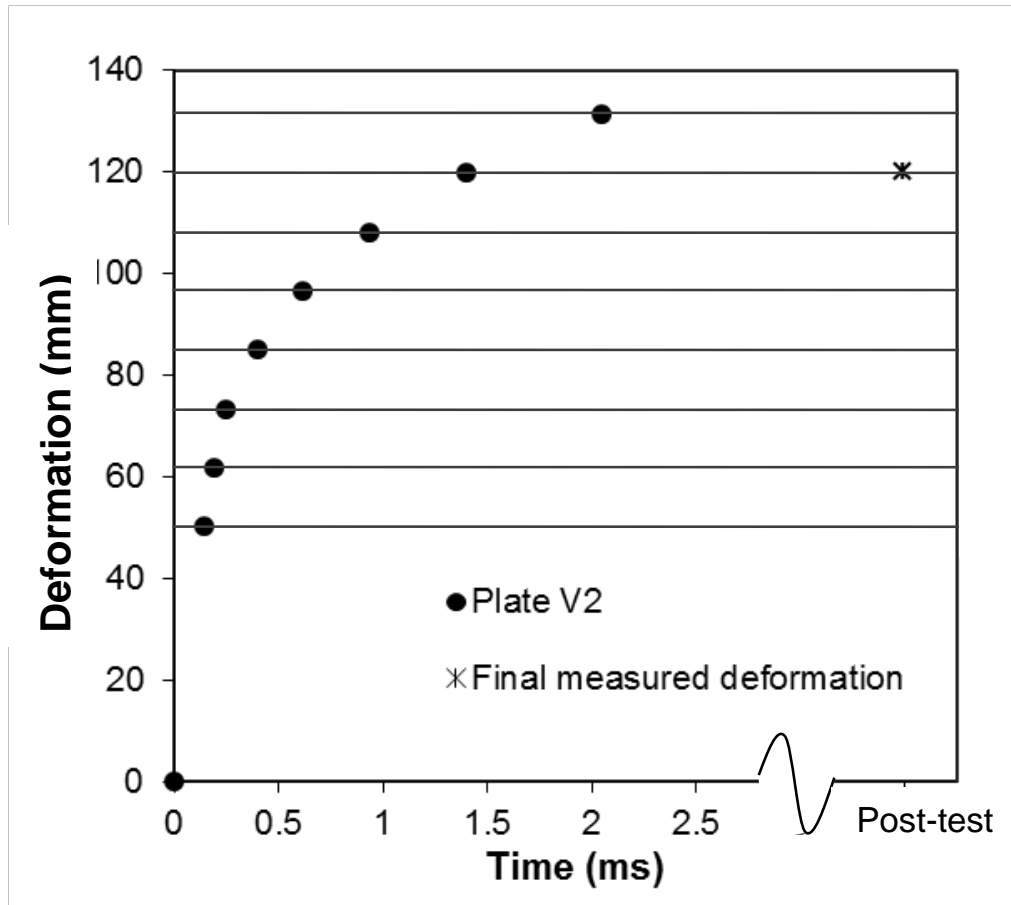

Figure 3.8 Deformation wire results for test V2. The horizontal lines represent the wire locations. 


\subsection{Numerical modelling}

Numerical simulations of the preliminary blast experiments were performed in order to validate the numerical model. Sensitivity studies, including mesh discretisation, grading and scheme (i.e. shell or hex elements) were performed in order to ensure a mesh-independent result while minimising the required computational time.

\subsubsection{Numerical modelling approach}

To model the validation experiments, a two-step approach was used to reduce the calculation time. In the first step, the detonation and initial expansion of the explosive was modelled using an $80 \mathrm{~mm}$ one dimensional (1D) spherically symmetric Euler model with $0.5 \mathrm{~mm}$ elements. This was achieved by modelling a wedge in AUTODYN, as shown in Figure 3.9. The wedge was filled with the material model for pentolite to a radius of $41.5 \mathrm{~mm}$, to match the experimental charge geometry. In the experiment, the detonation is symmetric until the shock interacts with the plate, which occurs at a radius of $77.5 \mathrm{~mm}$ for tests $\mathrm{V} 1$ and V2, $60.5 \mathrm{~mm}$ for V3 and $61.5 \mathrm{~mm}$ for V5 and V6. The 1D simulations were run until the radius of the blast wave was $77 \mathrm{~mm}$ for $\mathrm{V} 1$ and $\mathrm{V} 2$, and $60 \mathrm{~mm}$ for V3, V5 and V6, i.e. just before the blast wave interacts with the plate. Once they had reached the required blast radii, the 1D models were remapped onto a 3D Euler grid (Figure 3.10) with the charge centre positioned at the stand-offs given in Table 3.2. Note that V4 was not modelled as the plate ruptured, and developing a suitable failure model was outside the scope of this research. 


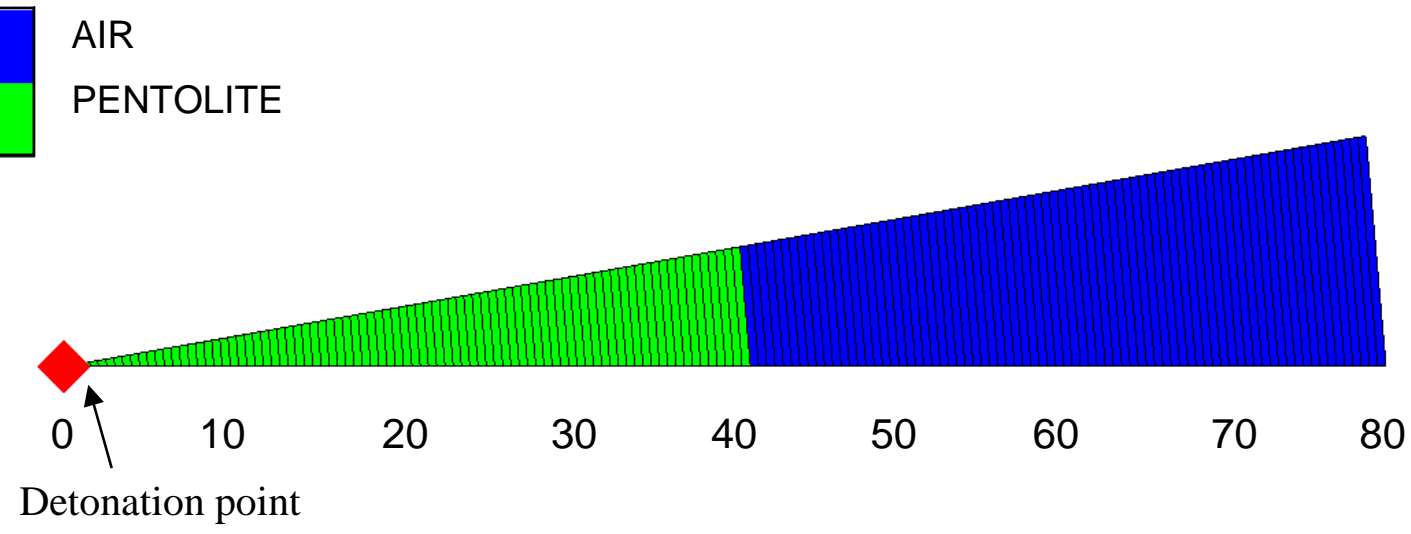

Figure 3.9 The initial 1D Euler wedge setup used to model pentolite detonation as a function of radius (mm) from the detonation point (red diamond).

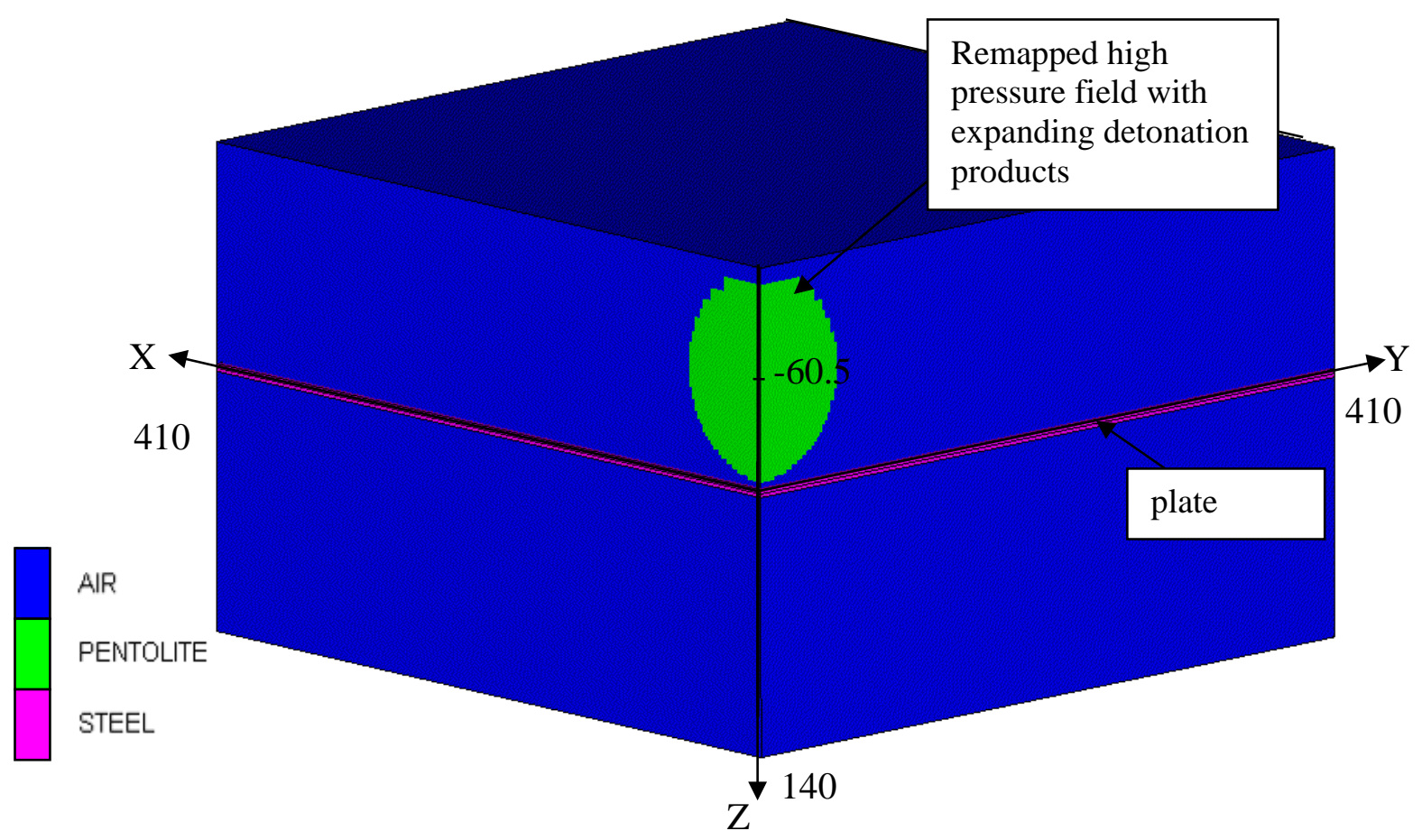

Figure 3.10 3D Euler model setup for an air-blast on a steel plate in AUTODYN, for test V3 (mesh elements not shown here). The dimensions are in millimetres and refer to the centre of detonation $(60.5 \mathrm{~mm})$ and the mesh boundaries, with the origin located at the centre of the steel plate. 


\subsubsection{Material modelling}

The ideal gas and Jones-Wilkins-Lee (JWL) equations of state were introduced in the literature review. These material models were used to model the air and the explosive (pentolite), respectively. The material model constants were taken from the AUTODYN material library. In the near-field of a blast event, the surrounding air plays a negligible role in the loading of surrounding structures, which is dominated by the flow of the detonation products. Hence, any inaccuracies which may be introduced by the use of an ideal gas equation of state for air are negligible.

The D36 plate material was modelled using a Johnson-Cook strength model for mild steel (Balden and Nurick, 2005), with an increased yield strength of 411 MPa based on tensile measurements by Hammond (Hammond, 2000). The Johnson-Cook strength model parameters used for the D36 steel are shown in Table 3.3.

Table 3.3 Johnson-Cook material model parameters used to model D36 steel (Balden and Nurick, 2005).

\begin{tabular}{ll}
\hline Johnson-Cook Parameter & Value \\
\hline Yield Stress, $\sigma_{0}$, & $411 \mathrm{MPa}$ \\
Hardening Constant, $B$ & $570 \mathrm{MPa}$ \\
Hardening Exponent, $n$ & 0.36 \\
Strain Rate Constant, $C$ & 0.35 \\
Thermal Softening Exponent, $m$ & 0.55 \\
Melting Temperature, $T_{\text {melt }}$ & $1793 \mathrm{~K}$ \\
Ref. Strain Rate (/s), $\dot{\varepsilon}_{0}$ & 1 \\
\hline
\end{tabular}

The XLERPLATE 350 steel was modelled using the same Johnson-Cook strength model parameters as the D36 plate, however the yield stress was set to $360 \mathrm{MPa}$, 
which is the guaranteed minimum yield strength for the XLERPLATE 350 steel (Bluescope Steel, 2009). Tensile tests of XLERPLATE 350 steel were performed at a later stage for the more accurate modelling in Chapter 5. For the validation experiments, the difference between using value for yield stress from literature (360 MPa) and the experimentally measured value (366 MPa) was negligible (less than $1 \%$ difference in residual deformation).

The polyurea was modelled using a 2-parameter Mooney-Rivlin model. The parameters used here were $C_{10}=875.2 \mathrm{kPa}, C_{01}=6321.3 \mathrm{kPa}$ and $\mathrm{d}=4 \times 10^{-7} \mathrm{kPa}^{-1}$, which were provided by the Naval Surface Warfare Centre, Carderock Division (NSWC-CD) for a similar (but not identical) polyurea from Roland et al. (2007) and Amirkhizi et al. (2006). The parameters were derived from test data generated at strain rates over $300 \mathrm{~s}^{-1}$ to reflect the high strain rates under blast loading. Figure 3.11 shows how the fitted tensile stress-strain curve compares with data from Roland et al. (2007). The fit appears to be reasonable at high strain rates (over $573 \mathrm{~s}^{-1}$ ), however, does not fit well at lower strain rates. The model exhibits slightly lower elastic stiffness compared to the data from Roland et al. (2007) at strains less than 0.5, and slightly higher stresses are seen in the model for strains between 0.5 and 3. Above strains of 3.5, the model quickly loses accuracy. This could be improved by fitting a 3-parameter Mooney-Rivlin model to capture the inflection; however for strains of less than 3 the 2-parameter model is sufficient. The model was considered a reasonable fit for high strain rate problems and was selected for validation against experimental results by Ackland et al. (2007). 
Chapter 3. Preliminary Experiments and Numerical Validation.

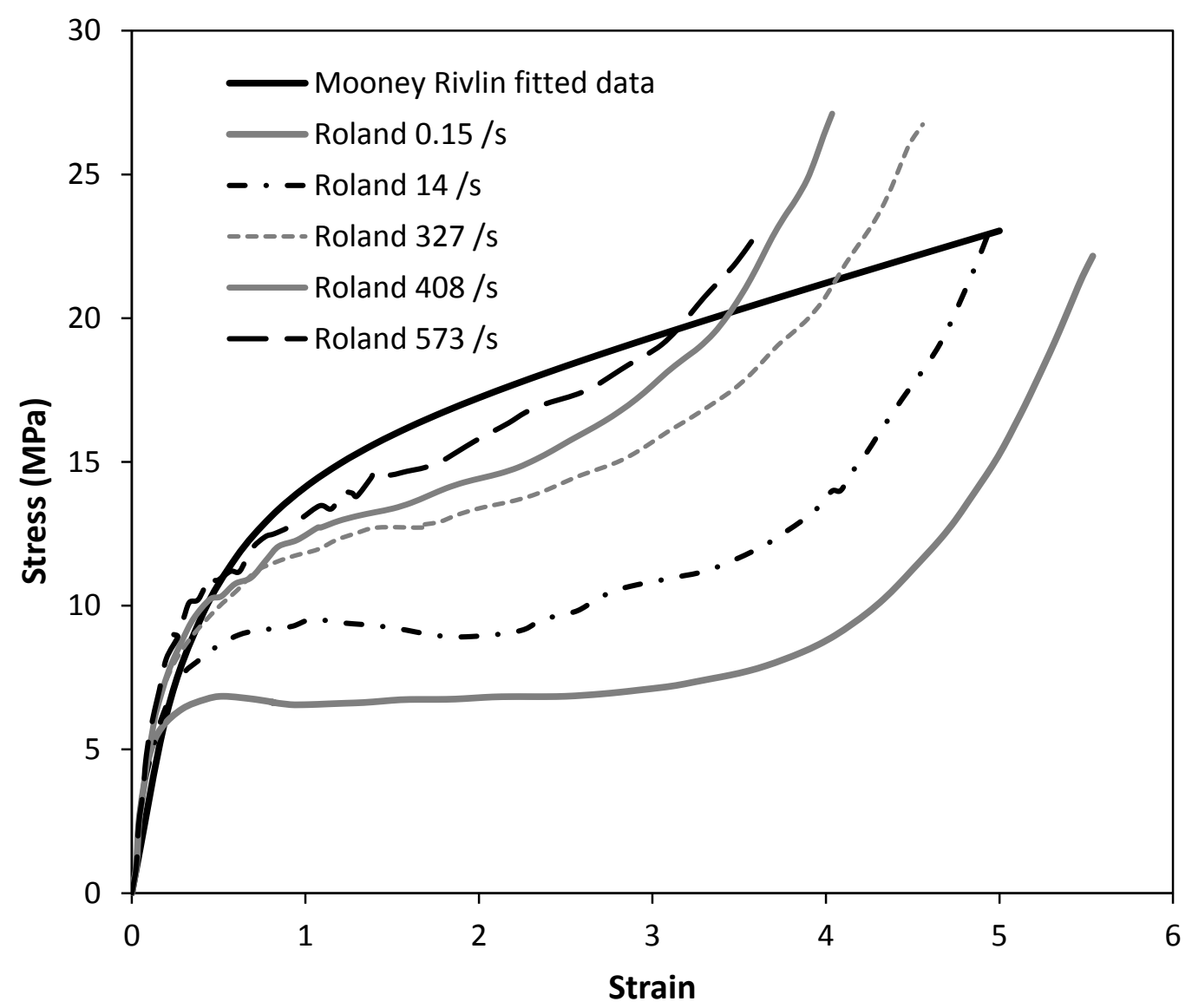

Figure 3.11 Fitted stress strain data using a 2-parameter Mooney-Rivlin fit compared with experimental data from Roland et al. (2007).

\subsubsection{Mesh discretisation of the steel plate}

The mesh discretisation was simplified by dividing the problem into three parts: mesh discretisation of the plate; mesh discretisation of the polyurea; and mesh discretisation of the Eulerian air grid. For the mesh discretisation of the plate, the problem was simplified by applying an initial velocity to a central area of the plate. This was not intended to accurately replicate the experimental blast load on the plate, but rather as a simple load to study the plate response for the mesh discretisation study, whilst ensuring a short computation time. It is recognised that this method will not capture the through-thickness stress wave in the plate, as would be expected as a result of an air blast, however it is sufficient to capture the bending and stretching of the plate under an impulsive load. For the polyurea coating, an impulsive load on the plate will 
generate a through-thickness stress wave in the polyurea, due to the compression force applied to the top surface of the polyurea.

Two types of element were considered to simulate the steel plate during this study: quadrilateral shell elements and Lagrangian hex elements. In each case a plate thickness of $4 \mathrm{~mm}$ was modelled to match the experimental plate thickness. To simulate the thickness of the plate using shell elements, the elements were assigned a thickness of $4 \mathrm{~mm}$. To simulate the plate thickness using hex elements, four elements of $1 \mathrm{~mm}$ thickness were used through the plate thickness.

A mesh discretisation study was conducted to determine a suitable element size to use later in the numerical modelling of the experiments. The numerical models were created in 3D with symmetry in the $\mathrm{X}$ and $\mathrm{Y}$ planes to significantly speed up the calculation time. For this exercise, the dimensions of the quarter plate were $400 \mathrm{~mm} \times 400 \mathrm{~mm}$. The non-symmetry boundaries of the plate were fixed by setting all translational and rotational velocities to zero at the external nodes to represent the clamped boundary.

An initial velocity of $150 \mathrm{~m} / \mathrm{s}$ was applied to a square section at the centre of the plate to simulate an impulsive load. The load was chosen so that the final plate deformation was similar to the experimental deformation.

The lateral dimensions of the plate elements were varied from $66.7 \mathrm{~mm} \times 66.7 \mathrm{~mm}$ down to $8 \mathrm{~mm} \times 8 \mathrm{~mm}$. Figure 3.12 shows the grids for the extreme cases. For each model the peak plate deformation at a point on the grid was determined using a gauge in AUTODYN. When gauges are applied to shell elements in AUTODYN, they are added to the centre of the element. Because of this, the location was chosen as shown in Figure 3.12 rather than at the plate centre, so that it would be in the same position for each mesh. The peak deformations at the gauges were compared to find the element size at which the deformation converged. 

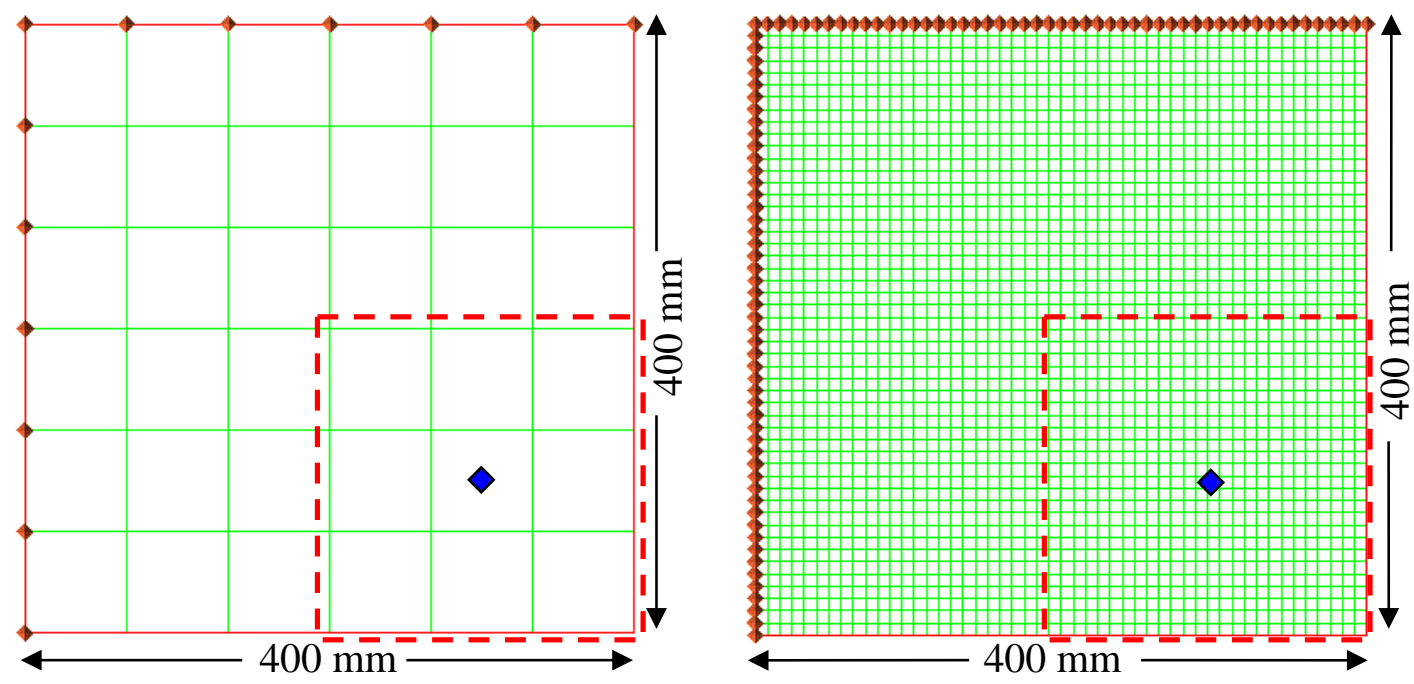

Figure 3.12 Two of the meshes used for the mesh resolution study of a steel plate under impulsive loading. The coarsest mesh is shown on the left, and the finest mesh on the right. The blue diamonds indicate gauge positions. A velocity of $150 \mathrm{~m} / \mathrm{s}$ was applied to the nodes within the red dashed line.

Figure 3.13 shows the results for the plate deflection for various grid sizes using shell elements. It was found that the solution converged with 34 elements along an edge, or $12 \mathrm{~mm} \times 12 \mathrm{~mm}$ elements. It was found that the point with only 6 elements along the edge also agreed with the convergent solution, however this is believed to be a spurious result. It is important to avoid using elements of this size because a small change in the model setup could cause a significant change in the plate deformation result. 
Chapter 3. Preliminary Experiments and Numerical Validation.

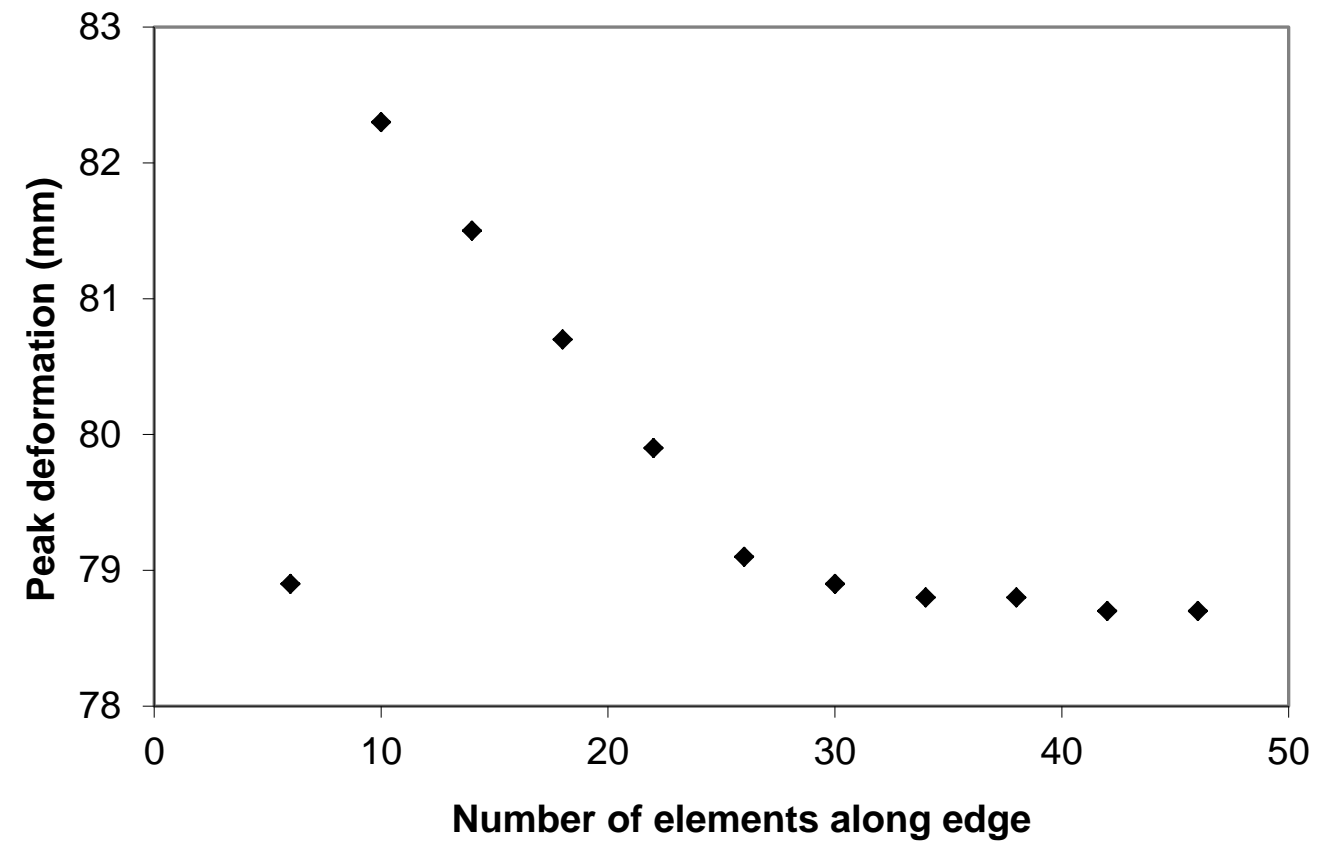

Figure 3.13 Effect of mesh resolution on the peak residual deformation of a plate under impulsive loading using shell elements.

Figure 3.14 shows the results of plate deflection for various grid sizes using hex elements. The solution converged with 25 elements along an edge, or $16 \mathrm{~mm} \times 16 \mathrm{~mm}$ elements of $1 \mathrm{~mm}$ thickness. 
Chapter 3. Preliminary Experiments and Numerical Validation.

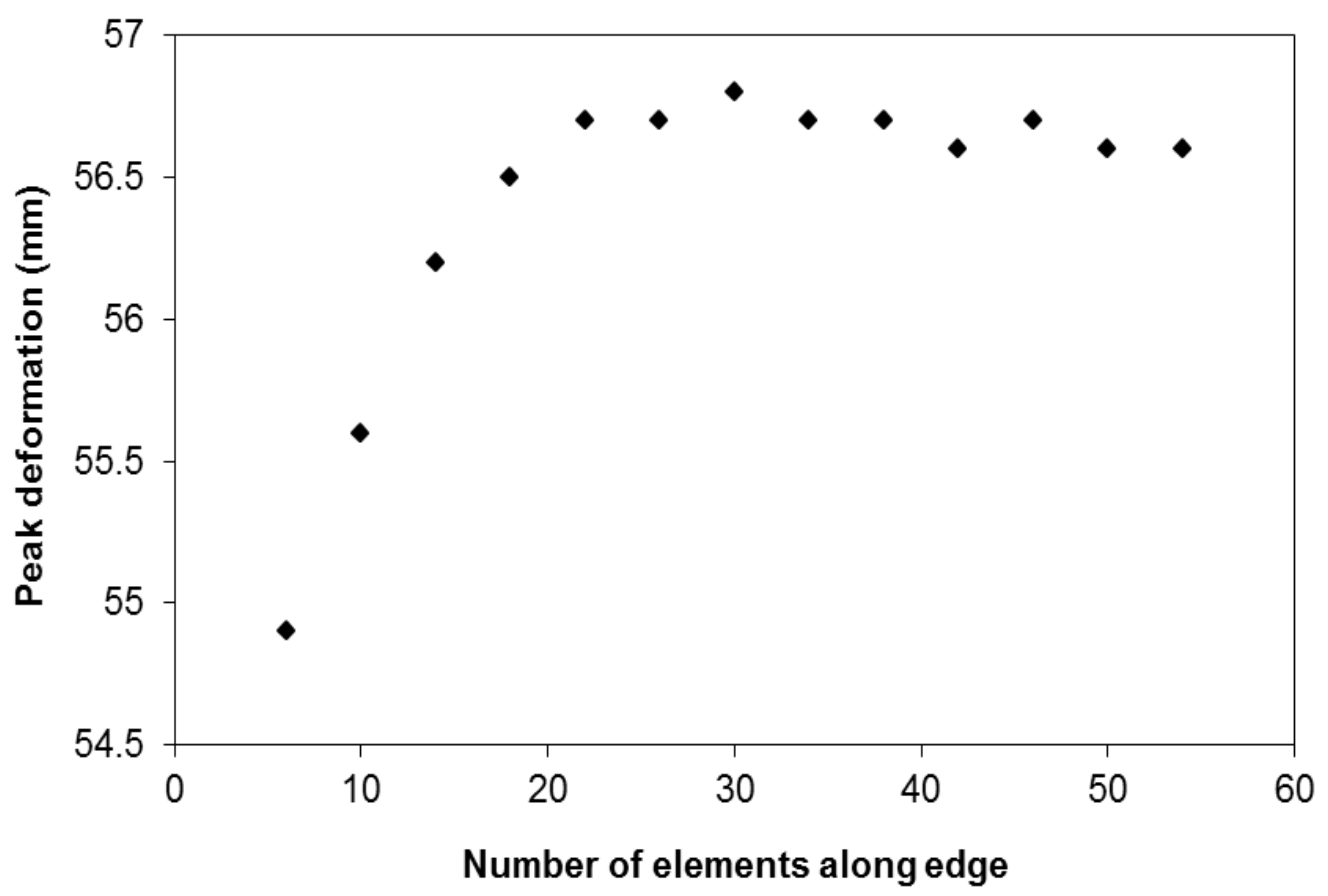

Figure 3.14 Effect of mesh resolution on the peak residual deformation of a plate under impulsive loading using hex elements (4 elements through thickness).

A simulation was also run using eight hex elements through the thickness of the plate (0.5 mm thick elements) and 38 elements along an edge to assess whether increasing the number of elements though the thickness of the plate changed the peak deformation. The peak deformation was found to be $56.7 \mathrm{~mm}$, which is the same solution that was achieved when four elements were used. Hence, it was not deemed necessary to use more than four elements through the plate thickness in future models.

A simulation was also run using 486 hex elements along an edge, or $0.82 \mathrm{~mm} \times 0.82 \mathrm{~mm} \times 1 \mathrm{~mm}$ elements, to observe the effect of using a very fine grid with an aspect ratio close to unity. The resulting peak deformation was $56.0 \mathrm{~mm}$. This is close to the apparent converged solution of $56.7 \mathrm{~mm}$.

In addition to the mesh resolution study, a weighted mesh was compared to an even mesh using each element type. A uniform $50 \times 50$ element mesh was used as a datum, while the weighted mesh had a total of 25 elements along each edge, with the 10 elements closest to the plate centre along each edge fixed at $8 \mathrm{~mm}$. In these 
simulations the gauges were positioned near the centre of the plate, as shown in Figure 3.15. An initial velocity of $200 \mathrm{~m} / \mathrm{s}$ was applied over a square section at the centre of the plate. This area was selected such that it was applied only to the elements which had the same dimensions $(8 \mathrm{~mm} \times 8 \mathrm{~mm}$ ) in both meshes, to ensure the same load on both plates.

The resulting deformation curves are shown in Figure 3.16 and Figure 3.17. Using shell elements, the peak deformations were $79.6 \mathrm{~mm}$ and $79.1 \mathrm{~mm}$ for the weighted and even mesh, respectively. This difference of $0.5 \mathrm{~mm}$ is relatively small, and it was decided that either mesh would be suitable for use in future studies. Using hex elements, the peak deformations were $50.5 \mathrm{~mm}$ and $50.9 \mathrm{~mm}$ for the weighted and even mesh, respectively. Figure 3.17 shows a slight variation between the deformation curves using hex elements, which can be seen by the difference in deformation values at $5 \mathrm{~ms}$.
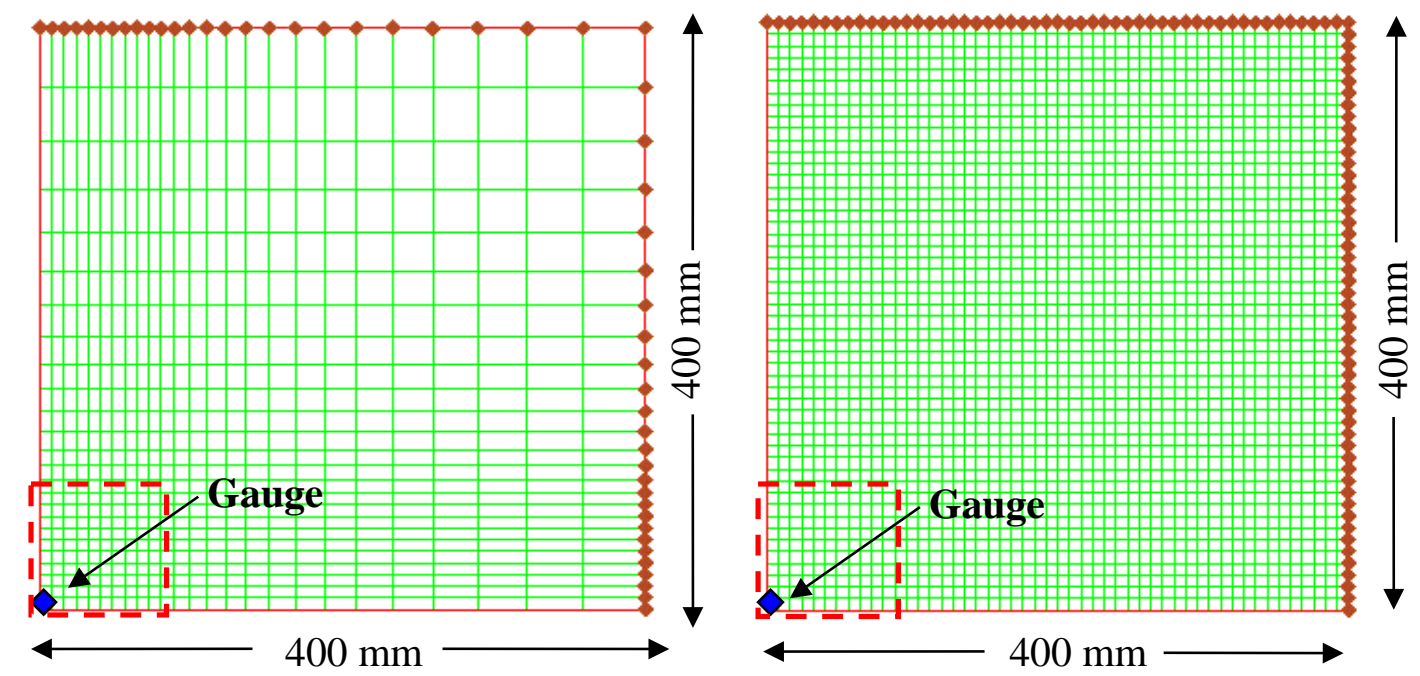

Figure 3.15 Meshes used to compare the effect of a weighted mesh (left) compared to an even mesh (right). The blue diamonds indicate gauge positions. A velocity of $200 \mathrm{~m} / \mathrm{s}$ was applied to the nodes within the red dashed line. 

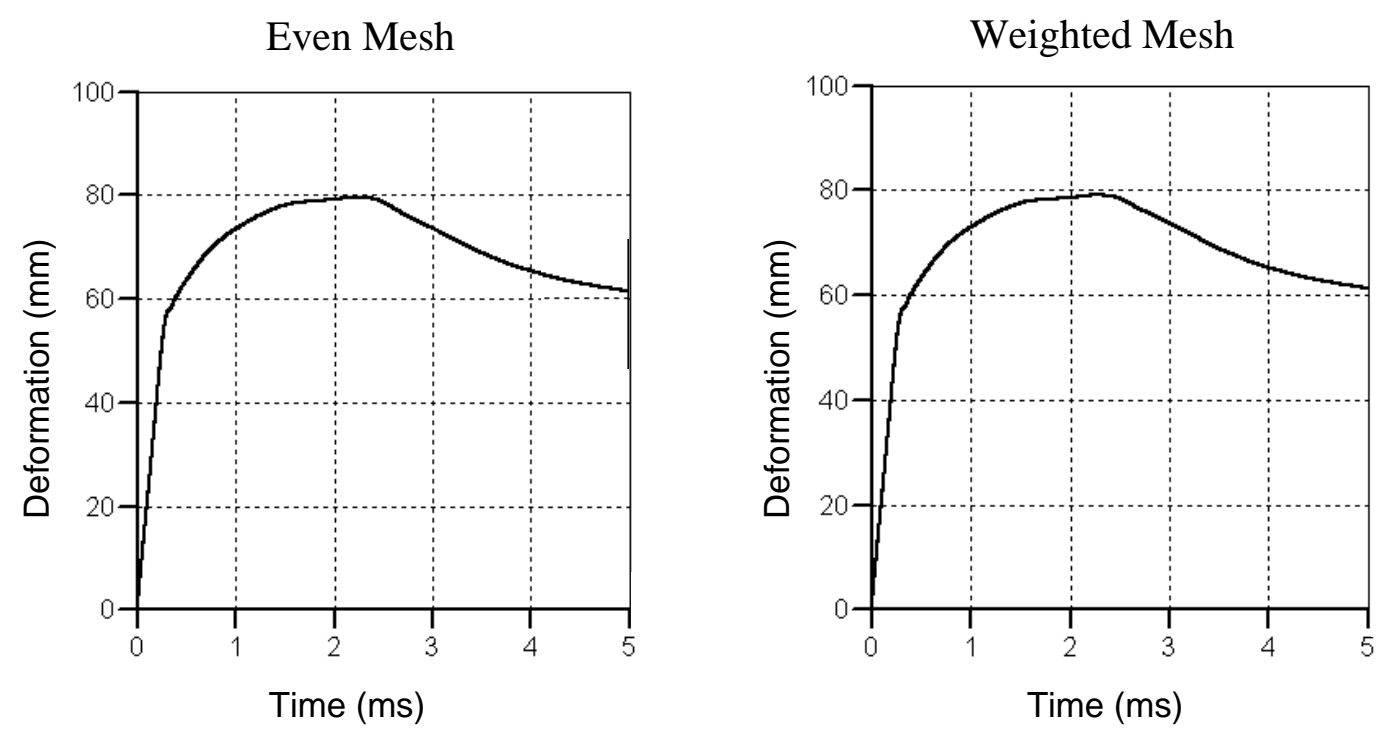

Figure 3.16 Comparison of deformation histories for a weighted mesh (right) compared with an even mesh (left), using shell elements.

Even Mesh

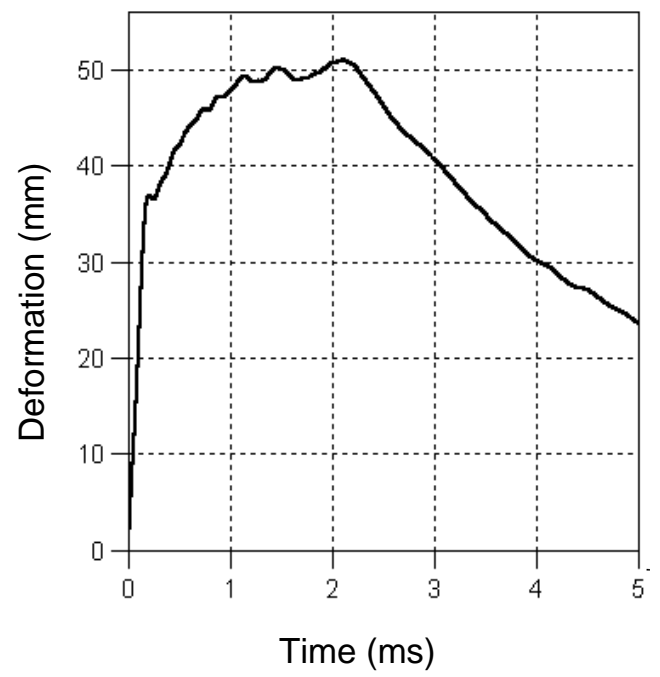

Weighted Mesh

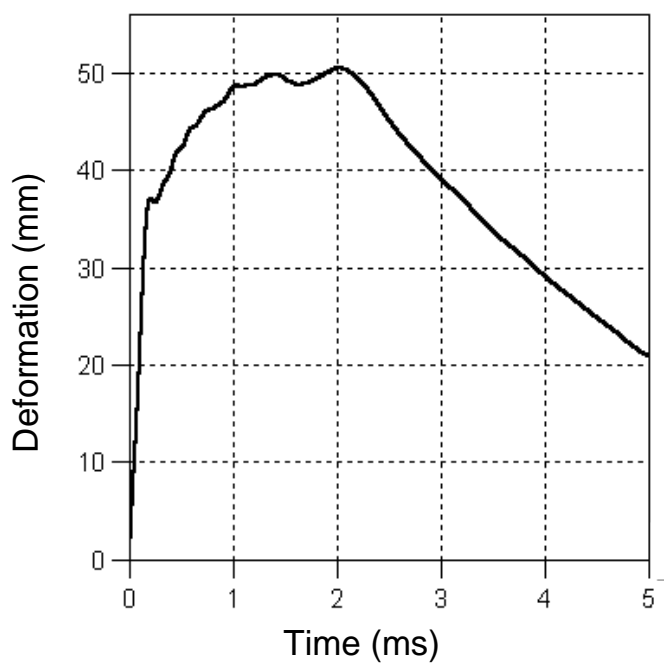

Figure 3.17 Comparison of deformation histories for a weighted mesh (right) compared with an even mesh (left), using hex elements.

The use of hex elements compared with shell elements yielded a significantly different peak transient deformation result for the same problem. This may have been due to a numerical problem known as shear locking occurring in the hex elements. Sun (2006) describes shear locking and discusses its effect on finite element 
calculations. The problem can arise when first order elements (i.e. with a single Gauss integration point) are subjected to bending moments. Figure 3.18 shows how an ideal element would deform under a pure bending moment. Note that the angle $\alpha$ remains at $90^{\circ}$ as predicted by classical beam theory (Gere and Timoshenko, 1997). When a firstorder element is used to simulate this situation, the edges are not able to bend to match the curves shown in Figure 3.18, but instead take the shape shown in Figure 3.19. This causes the angle $\alpha$ to no longer be a right angle, introducing an artificial shear stress. This is known as shear locking and may cause the element to lock or become overly stiff, and could have resulted in the reduced deformations as seen here.
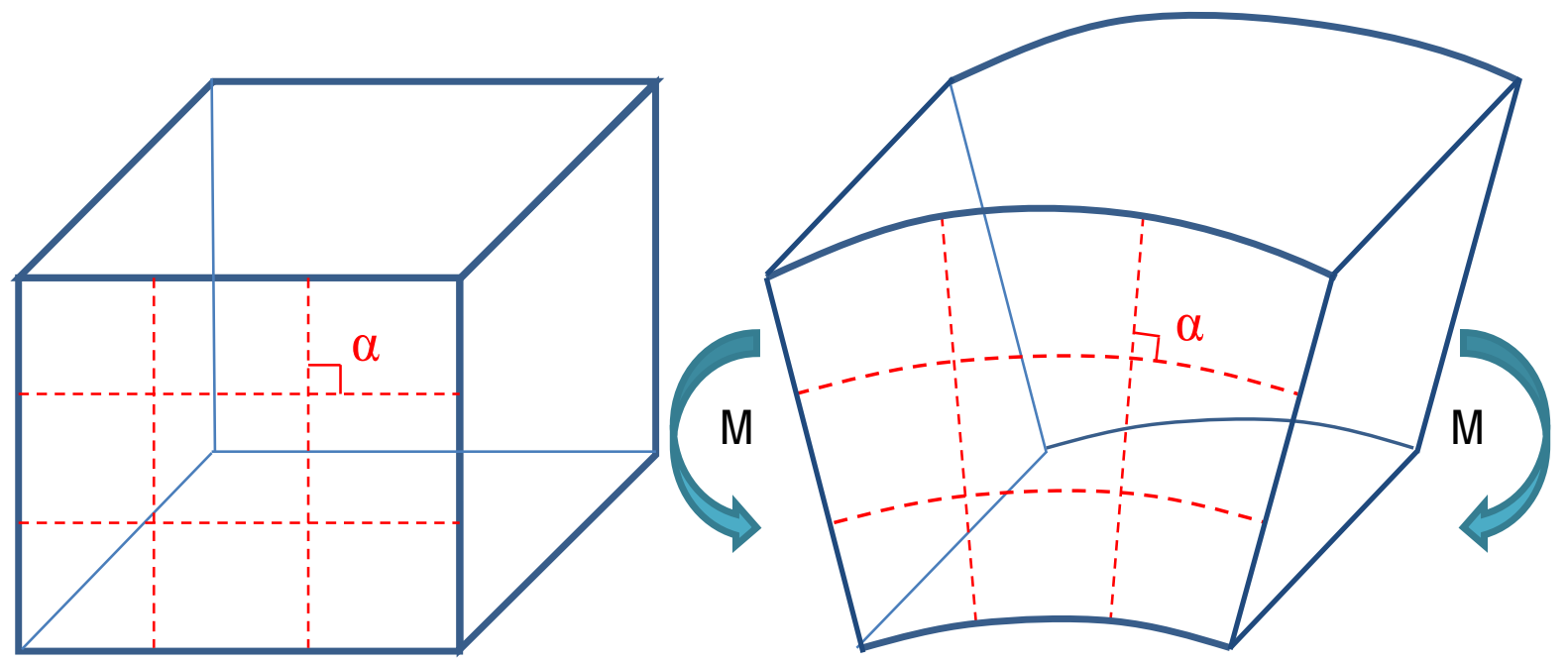

Figure 3.18 Geometry change of an ideal element under a pure bending moment.
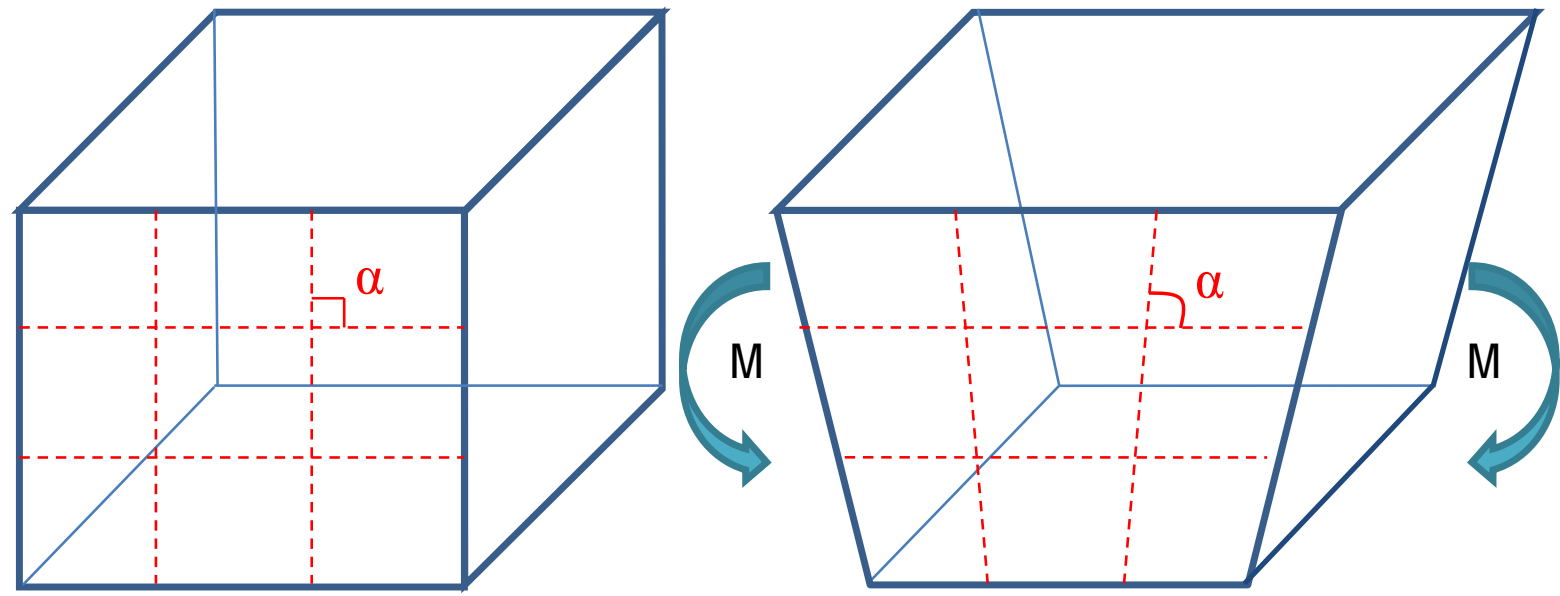

Figure 3.19 Geometry change of a fully integrated first-order element under a bending moment. 
The use of shell elements results in a significant reduction in computational time compared to using hex elements, which is an advantage when considering the element type for future work. However, given the difference in results using different element types, both methods were trialled initially to determine which method more accurately predicted the experimental results.

\subsubsection{Mesh discretisation of the polyurea coating}

A mesh resolution study was also conducted to investigate the element size required to accurately model the polyurea coatings. The mesh resolution study was set up in a similar way to the bare plate study, again using a $400 \mathrm{~mm} \times 400 \mathrm{~mm}$ plate. Again, the load on the polyurea-coated plate was approximated to an impulsive load, which was simulated by applying an initial, instantaneous velocity of $200 \mathrm{~m} / \mathrm{s}$ to the plate. The plate was modelled using shell elements to minimize the calculation time. The initial velocity and gauges were applied to the same locations as shown in Figure 3.12.

The $8 \mathrm{~mm}$ thick polyurea was modelled using 8-noded Lagrange hex elements and a 2-parameter Mooney-Rivlin material model with the constants provided in Section 3.3.2. Hex elements were chosen rather than shell elements for the polyurea, as it was assumed that the through-thickness stresses in the polyurea would be an important factor affecting its behaviour.

For the initial mesh resolution study, cubic hex elements were used to model the polyurea. The first simulation used $8 \mathrm{~mm}$ elements, which were reduced for each subsequent simulation down to a size of $2 \mathrm{~mm}$. The dimensions of the plate elements were selected so that the nodes lined up and the two parts could be joined. The results are shown in Figure 3.20. At the time of this initial study, computational limitations did not allow a $1 \mathrm{~mm}$ element size to be simulated (using a workstation with a dualcore AMD Opteron 3.2 GHz CPU and 16 GB of RAM). However, a later result using a $2.6 \mathrm{~mm} \times 2.6 \mathrm{~mm} \times 1 \mathrm{~mm}$ element size is shown in Figure 3.20. The results show that as the element size was reduced, the peak deformation was also reduced. This is consistent with the results for the shell element discretisation shown in Figure 3.13, and has been seen in other DSTO studies using shell elements to simulate the dynamic response of plates to impact and blast loads. Although convergence was not observed 
for the polyurea-coated plate, it appears as though the solution may be approaching a converged solution of around 90 to $91 \mathrm{~mm}$ (by extrapolating towards an infinitely small element size of side length $0 \mathrm{~mm}$ ). The non-convergence may be due to the polyurea elements not being small enough to capture the through-thickness stress wave accurately. However, the results are within approximately $2 \%$ of the apparent converged solution using $2 \mathrm{~mm}$ elements, which is considered reasonable.

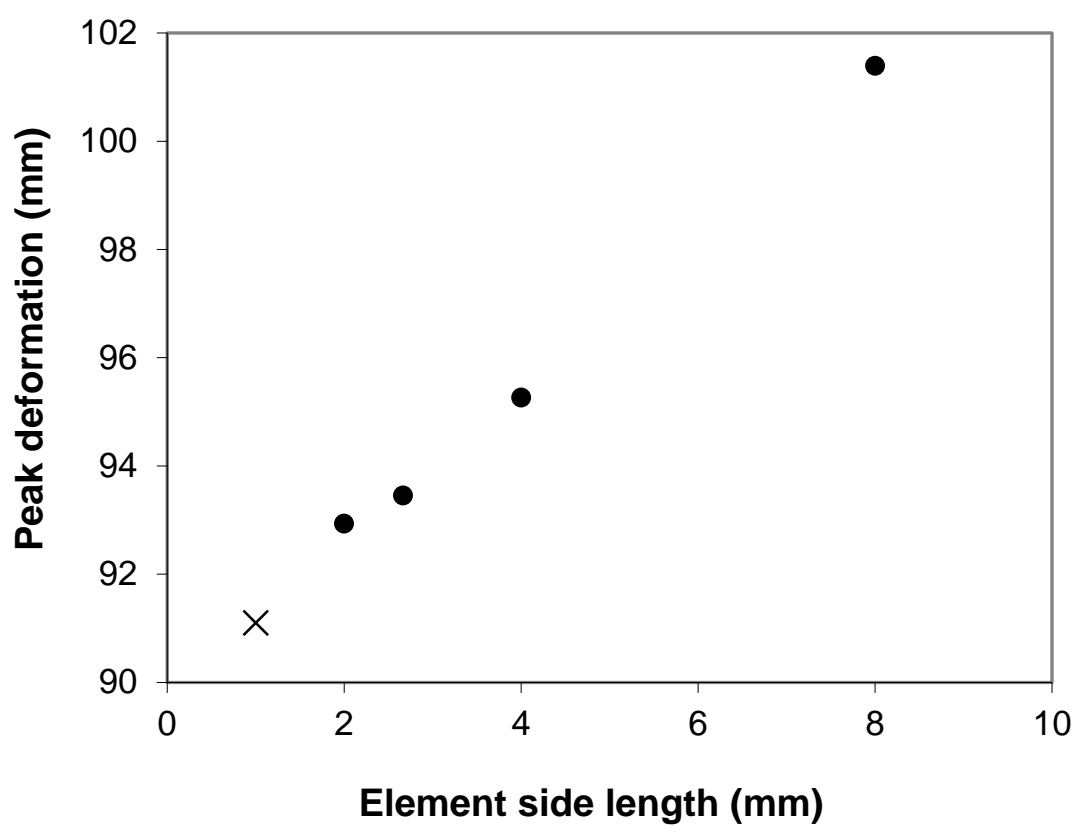

Figure 3.20 Effect of polyurea hex element size on peak residual plate deformation. The ' $X$ ' shows the result for a $2.6 \mathrm{~mm} \times 2.6 \mathrm{~mm} \times 1 \mathrm{~mm}$ element size.

A second convergence study was conducted using a constant element thickness of $2 \mathrm{~mm}$ while the length and width of the elements was varied. This was then repeated for element thicknesses of $1.5 \mathrm{~mm}$ and $1 \mathrm{~mm}$. Figure 3.21 shows the results of this study. In each case the solution appears to converge when 50 elements are used along the edge of the plate, however each setup appears to converge to a different solution. This indicates that the solution has not truly converged, however supports the case that the solution is tending towards approximately 90 to $91 \mathrm{~mm}$. 
Chapter 3. Preliminary Experiments and Numerical Validation.

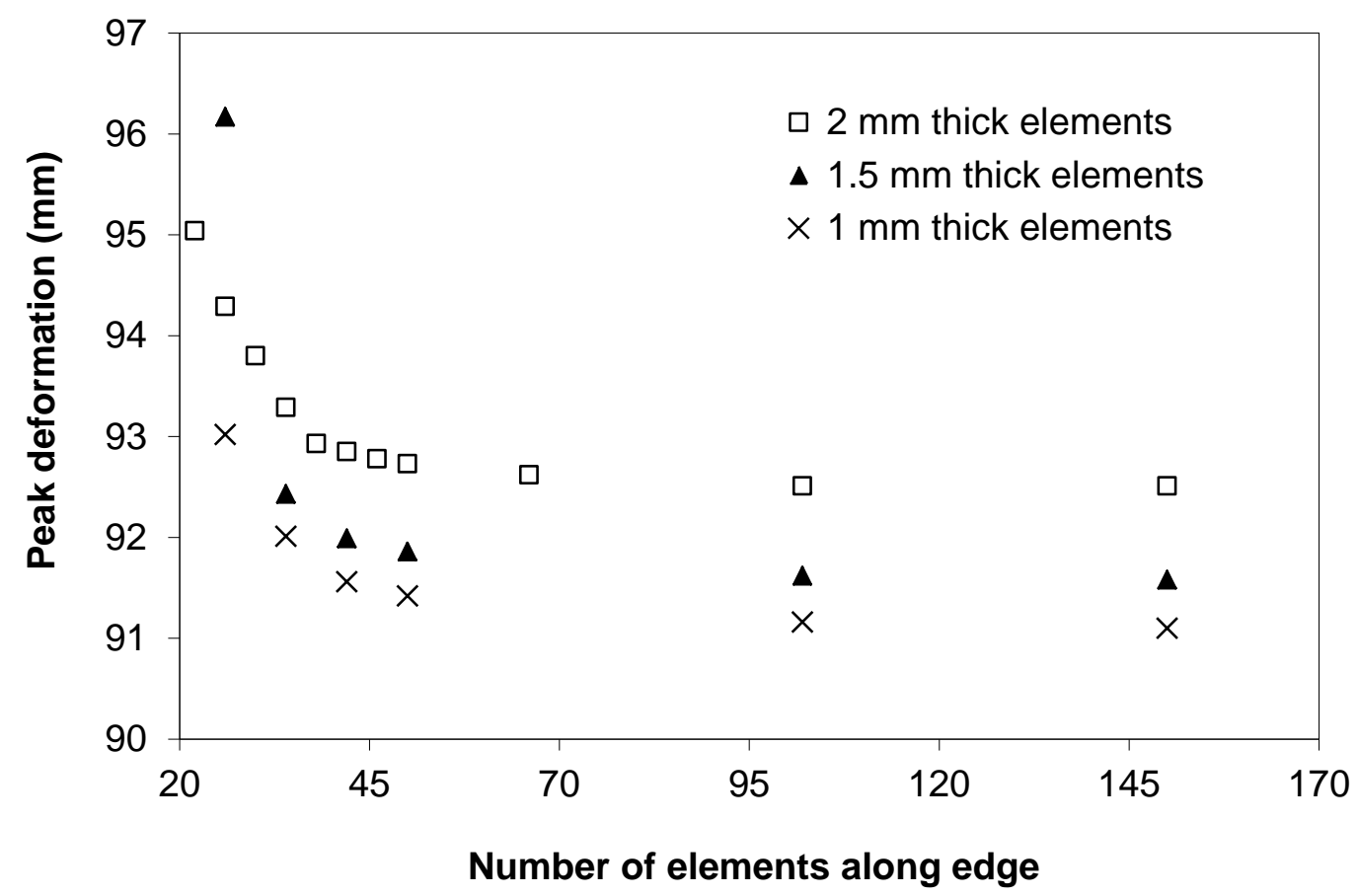

Figure 3.21 Effect of polyurea hex element dimensions on peak residual plate deformation.

Whilst full convergence was not seen, it was found that reasonable results can be achieved by having at least 50 elements along the edge of the plate (element side length of $8.2 \mathrm{~mm}$ ). Additionally, increasing the number of elements through the plate thickness gives better results.

\subsubsection{Mesh discretisation of Euler grid}

A mesh discretisation study was also conducted on the Euler grid to determine what size elements are required to model late stage expansion of the detonation products and the interaction with the target plate. A three dimensional (3D) rectangular Eulerian mesh was created with dimensions $200 \mathrm{~mm} \times 200 \mathrm{~mm} \times 100 \mathrm{~mm}$. Elements were 8-noded hex elements and symmetry was applied in the $\mathrm{X}=0$ and $\mathrm{Y}=0$ planes. The $\mathrm{Z}$ plane was a reflecting surface, to simulate a similar effect to the reflection off a plate. All the other surfaces were given a "flow out" boundary condition, which allows material to flow across the boundary. 
The initial detonation of the explosive was modelled as described earlier in Section 3.3.1, with a $77.5 \mathrm{~mm}$ stand-off from the plate to the charge centre. Figure 3.22 shows the model setup with the 1D wedge remapped onto the 3D Euler grid.

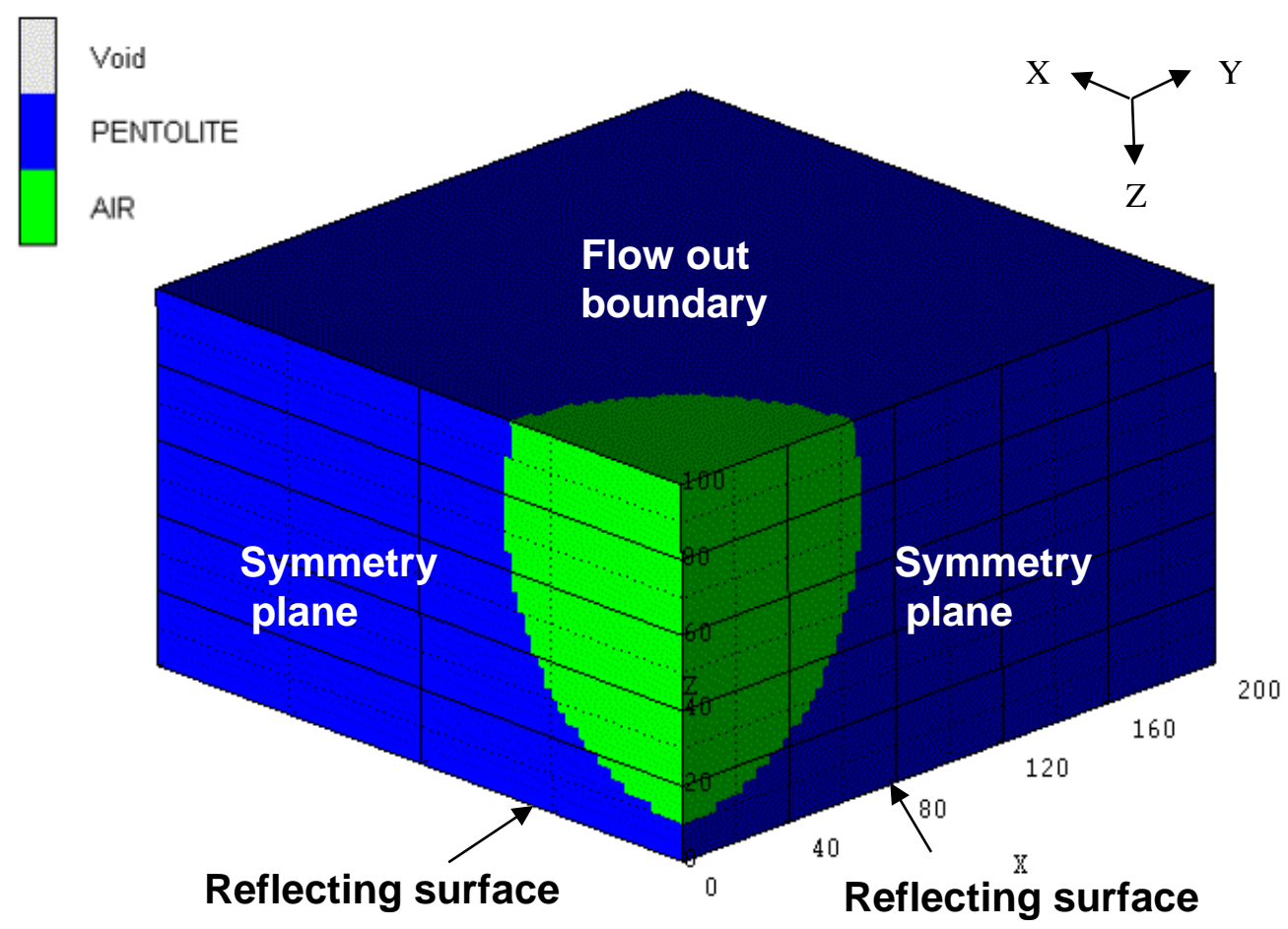

Figure 3.22 3D Euler model of air-blast after remapping pentolite detonation (mesh elements not shown here). Dimensions are in mm.

Five gauges were positioned along the diagonal of the reflecting surface to measure the reflected pressure. This is representative of the pressures that the steel plate would experience. Grid sizes of 4000, 32 000, 256000 and 864000 elements were used, and the pressures across the plate for each of the grids were compared. Weighted meshes were also considered, with sizes of 100 000, 337 000, and 800000 elements. Figure 3.23 shows an example of a weighted mesh, with elements concentrated about the centre of the reflecting surface, where the highest pressures were expected. Figure 3.24 shows the maximum dynamic pressures measured by each of the gauges used in the AUTODYN models. Dynamic pressures dominate the loading at this very close-in range, and hence, are representative of the load that will be experienced when a plate is used for the reflecting surface. 
Chapter 3. Preliminary Experiments and Numerical Validation.

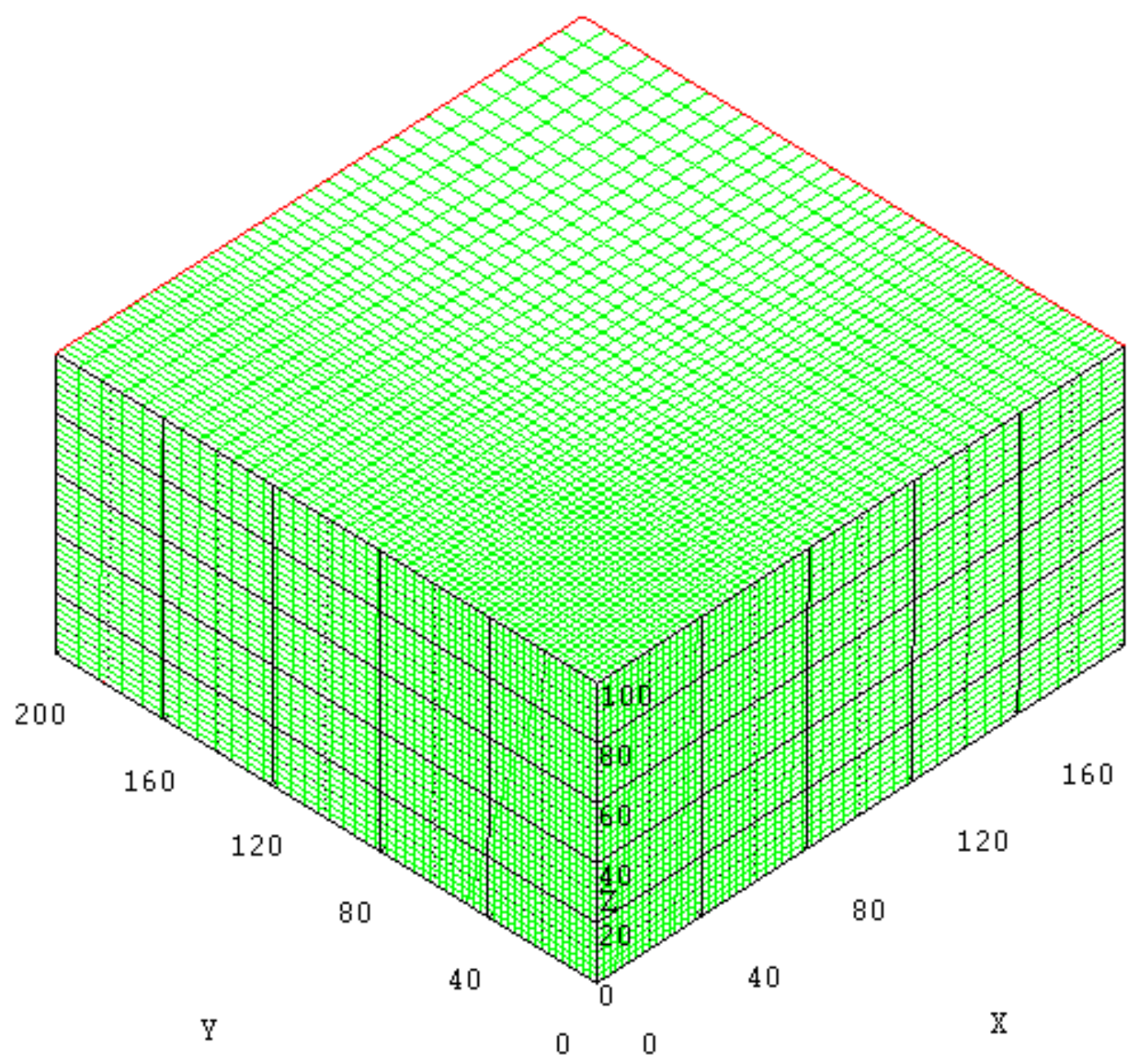

Figure 3.23 Weighted mesh investigated to model air-blast. Dimensions are in mm. 
Chapter 3. Preliminary Experiments and Numerical Validation.

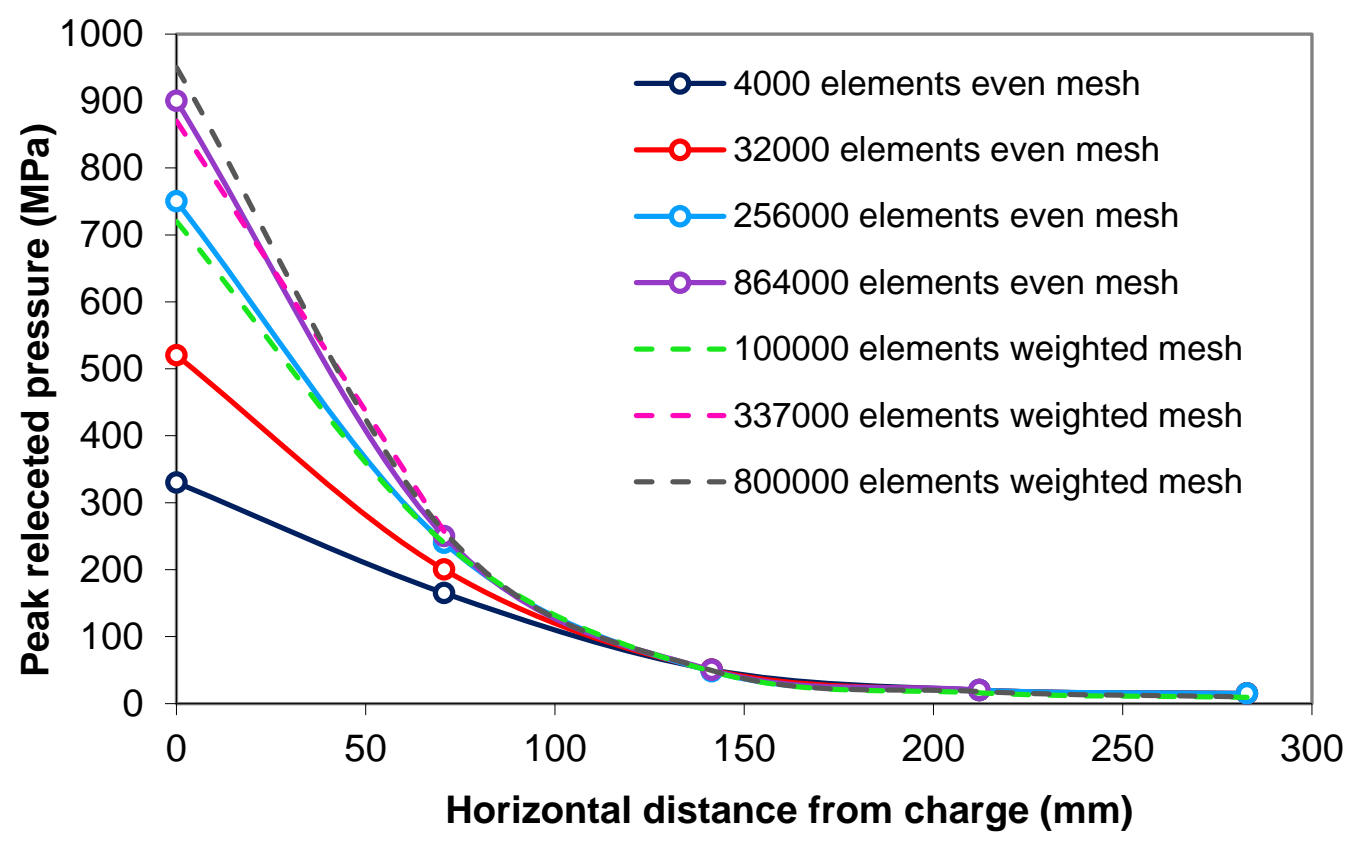

Figure 3.24 Peak reflected pressures measured along the reflecting surface for an air-blast, modelled using AUTODYN.

As can be seen in Figure 3.24, the weighted meshes require fewer elements than the even meshes to produce the same output. For example, the 256,000 element even mesh and the 100,000 element weighted mesh gave similar results for the pressure profile, as did the 864,000 element even mesh compared to the 337,000 element weighted mesh. Hence it was determined that a weighted mesh was clearly the best option for use in future models.

The finest weighted mesh had cubic elements of $1.25 \mathrm{~mm}$ side length near the centre of the reflecting surface, whereas the second finest weighted mesh had cubic elements of $1.67 \mathrm{~mm}$ side length. When considering the entire peak reflected pressure curve across the plate (Figure 3.24), the two curves are very similar, deviating only in the vicinity of the centre of the loading surface. Based on these results, it was decided that elements of no larger than $1.67 \mathrm{~mm}$ would be used in the area of near the centre of the reflecting surface. 


\subsection{Numerical simulation and comparison with the experimental results: bare steel plates}

Two models were created for comparison with the validation experiments V1 and V2, one using shell elements to model the plate and one using hex elements. A 4 mm thick plate was modelled using the Johnson-Cook material model with constants for Bluescope XLERPLATE 350 from Section 3.3.2. The air was modelled using a 3D Eulerian grid with dimensions of $410 \mathrm{~mm} \times 410 \mathrm{~mm} \times 250 \mathrm{~mm}$, discretised into 1,872,000 elements. Figure 3.25 shows the mesh, which was weighted so there were smaller elements where the highest pressures were expected. The elements in the vicinity of the plate centre were $1.5 \mathrm{~mm} \times 1.5 \mathrm{~mm} \times 1.5 \mathrm{~mm}$, which was selected based on the Euler grid mesh discretisation study. The $\mathrm{X}=0$ and $\mathrm{Y}=0$ planes were set as symmetry planes, and a "flow out" condition was applied to the remaining four surfaces of the grid.

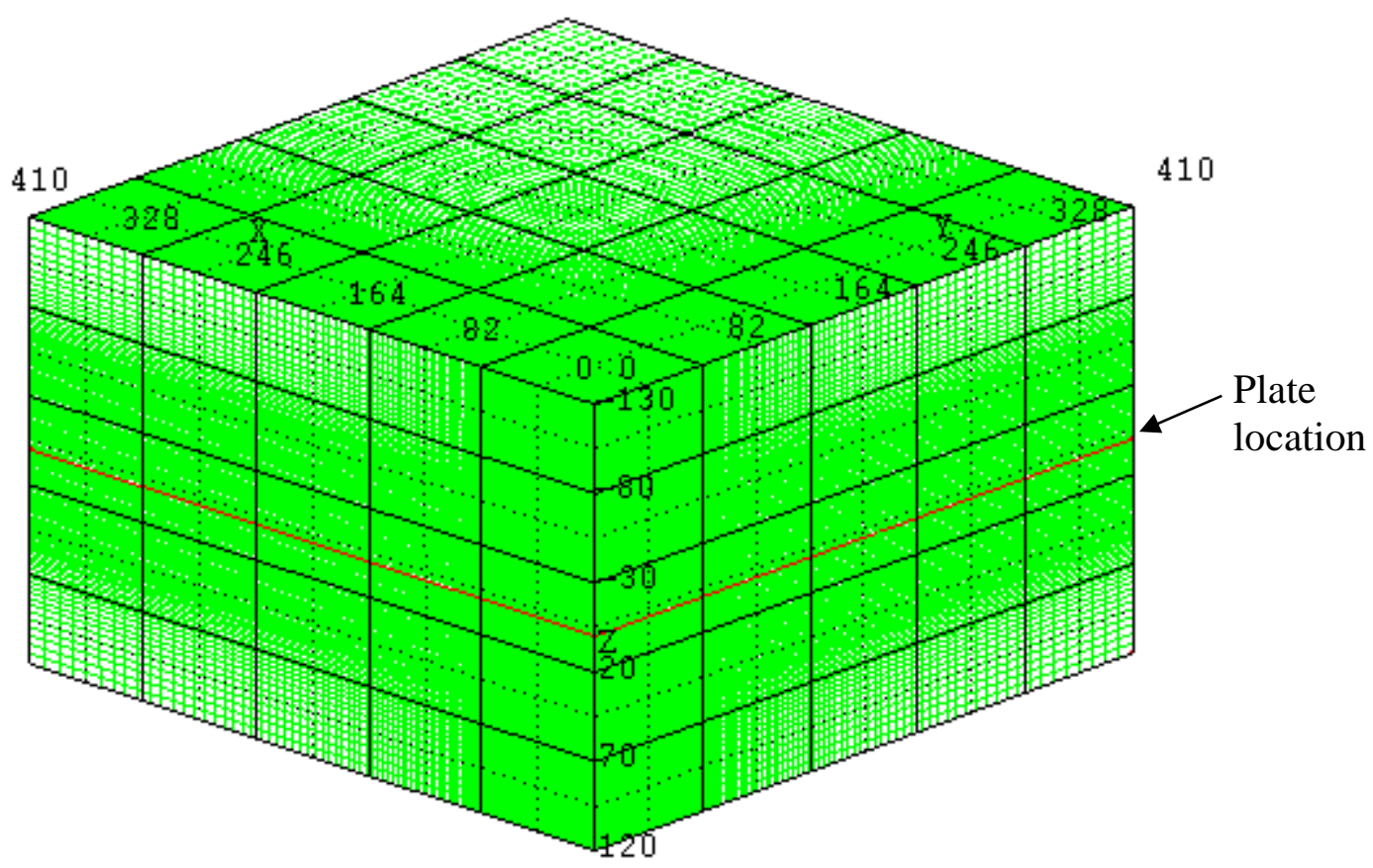

Figure 3.25 Mesh for air-blast on steel plate. The red line represents the location of the plate. In the solid green areas the mesh is too fine for the picture resolution. 
The air behind the plate was modelled to allow the plate to continue to be loaded as it deforms. As such, the grid must cover the entire space in which the plate is expected to move during the loading phase.

The explosive was simulated in the $1 \mathrm{D}$ wedge model with a diameter of $80 \mathrm{~mm}$ and discretised into $0.5 \mathrm{~mm}$ thick elements. This was run until the radius of the blast wave was $75 \mathrm{~mm}$, such that it was about to interact with the plate. At this point the $1 \mathrm{D}$ wedge was remapped onto the $3 \mathrm{D}$ grid with the charge centre positioned $77.5 \mathrm{~mm}$ above the centre of the front surface of the plate.

In the first model, the plate was modelled using $8.2 \mathrm{~mm} \times 8.2 \mathrm{~mm}$ shell elements of $4 \mathrm{~mm}$ (artificial) thickness. The second model used hex elements with dimensions $13.67 \mathrm{~mm} \times 13.67 \mathrm{~mm} \times 1 \mathrm{~mm}$, resulting in four elements through the plate thickness. The non-symmetry boundaries of the plate were fixed to represent the clamped edges by setting all translational and rotational velocities to zero at the nodes. The EulerLagrange interaction was set to fully coupled, to allow the air-blast and plate to interact.

Figure 3.26 shows an example of the material energy summary for the shell element model, where it can be seen that by around $0.3 \mathrm{~ms}$ the energy transfer can be considered complete. As such, at $0.3 \mathrm{~ms}$ after detonation, the air was removed from the model to increase calculation speed. The same method was used in the hex element model. 
Chapter 3. Preliminary Experiments and Numerical Validation.

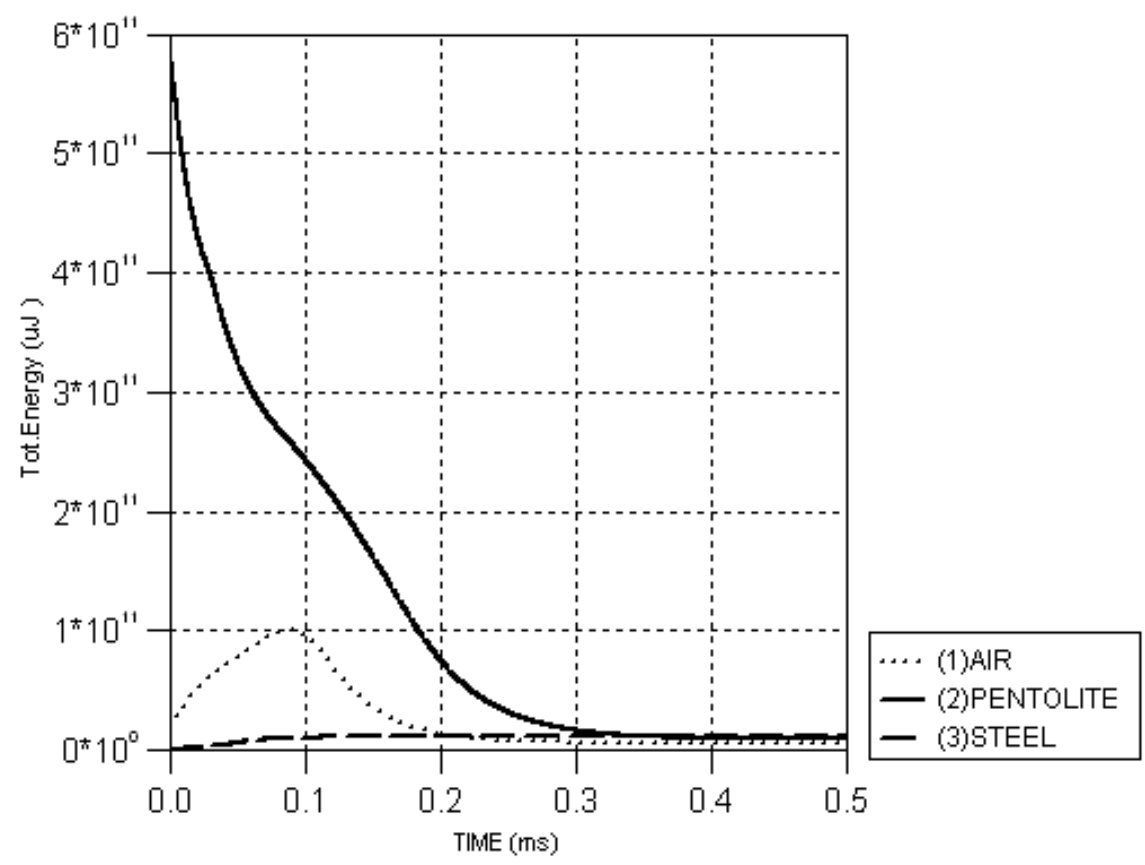

Figure 3.26 Material energy summary for blast loading on a steel plate.

Figure 3.27 shows the model as the blast begins to interact with the plate. The air is plotted in contours of pressure. Note that there is a region of very high pressure at the plate centre. This is due to the reflection of the blast wave off the plate at very close range. 


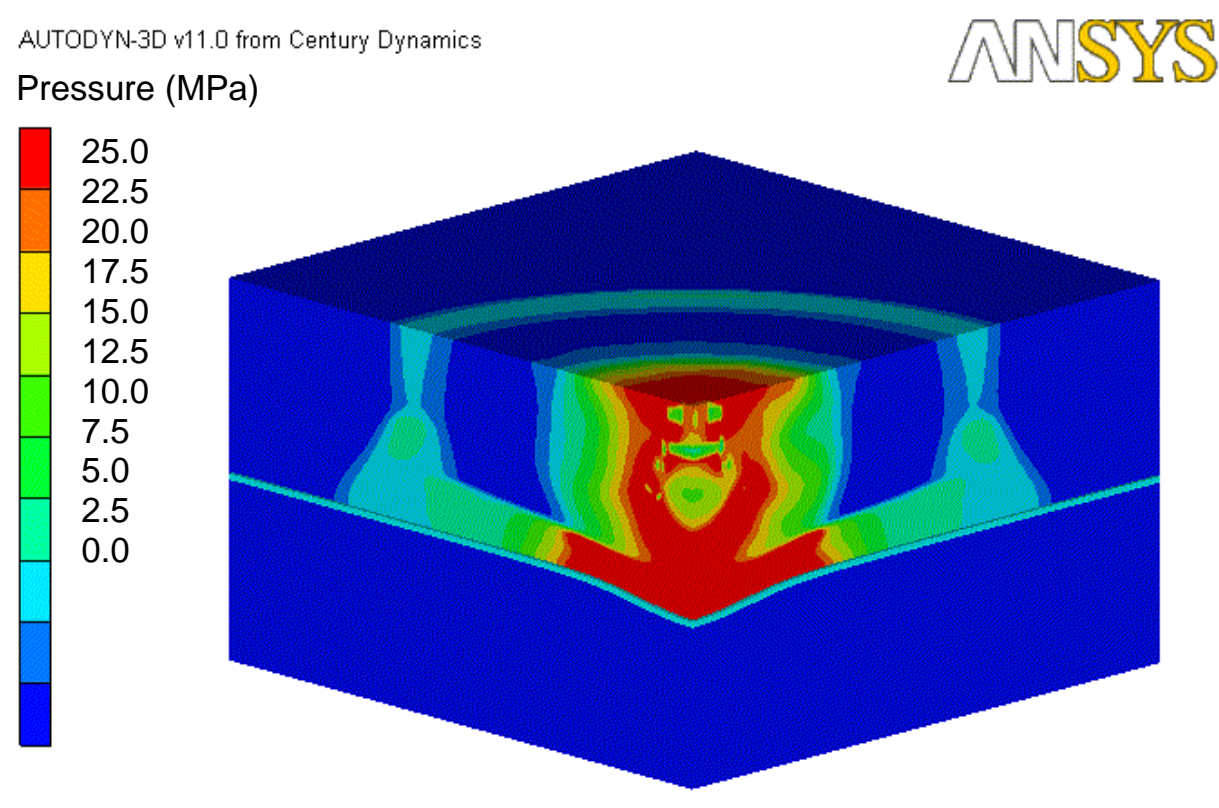

Figure 3.27 AUTODYN model of an Eulerian air-blast interaction with a Lagrangian steel plate at approximately 0.05 ms after detonation.

Figure 3.28 shows the deformation history of a gauge located at the centre of the plate. Note that a cyclic pattern can be seen from around $2 \mathrm{~ms}$ until $10 \mathrm{~ms}$. After $10 \mathrm{~ms}$ a static damping constant was applied to the model to allow the dynamic solution to converge to a state of stress equilibrium. This is an artificial condition which is required for the solution to converge, as the dynamic model does not simulate the damping of the plate in which its kinetic energy is transferred to the environment (mainly as heat). The static damping constant for optimal convergence, $R$, can be calculated using the time step, $\Delta t$, and the longest period of vibration for the system being analysed, $\omega_{\max }$ (ANSYS AUTODYN, 2009):

$$
R=2 \Delta t / \omega_{\max }
$$

This calculation resulted in a static damping constant of $5.5 \times 10^{-4}$ for the shell element plate and $7.3 \times 10^{-5}$ for the hex element plate. The final plate deformations for the shell element and hex element models are shown in Figure 3.29 and Figure 3.30 , respectively. 
Chapter 3. Preliminary Experiments and Numerical Validation.
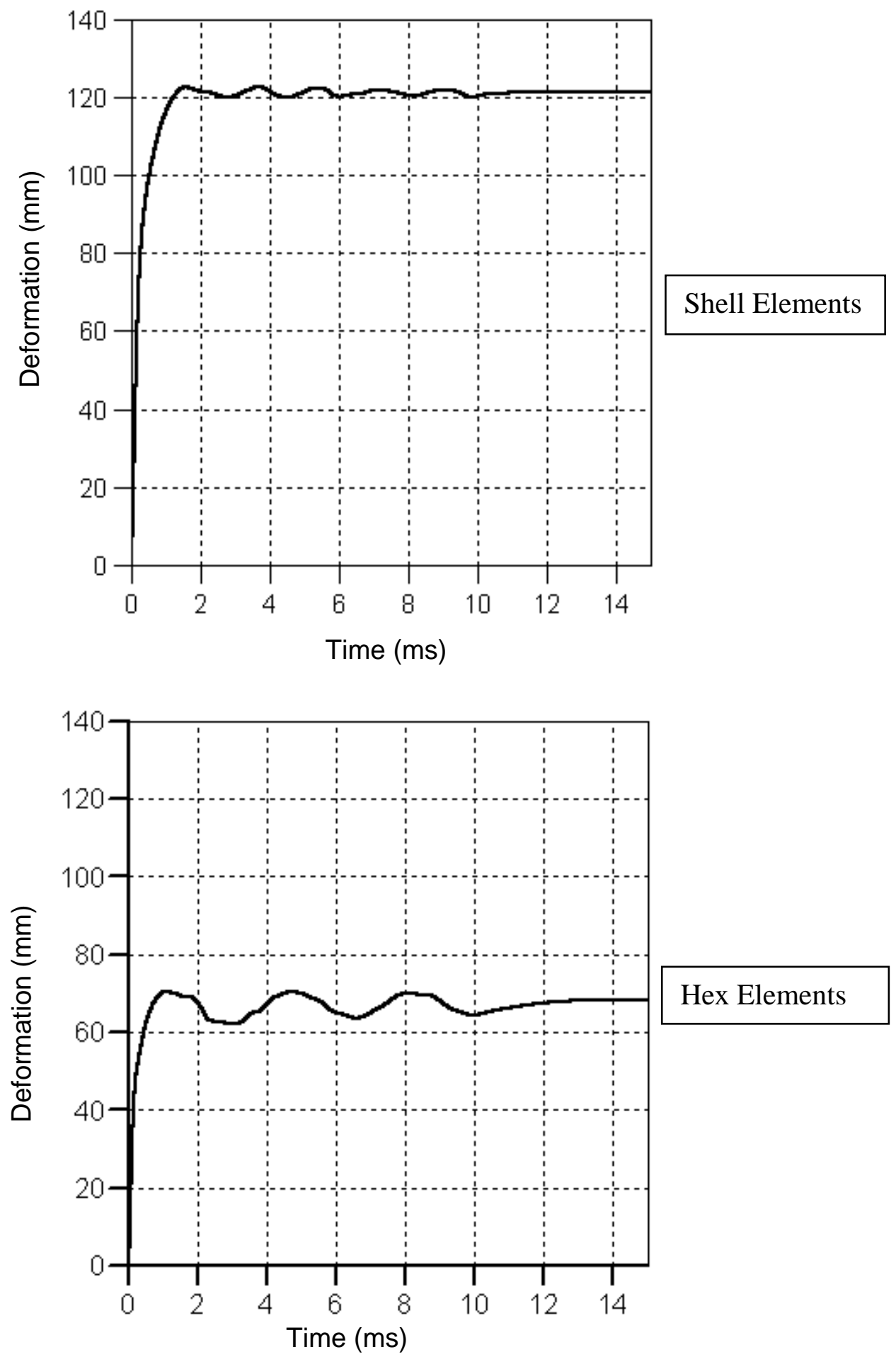

Figure 3.28 Deformation histories for a $4 \mathrm{~mm}$ steel plate under blast loading measured using gauges in AUTODYN. The top plot was generated from a model using shell elements and the bottom plot was generated using hex elements. 


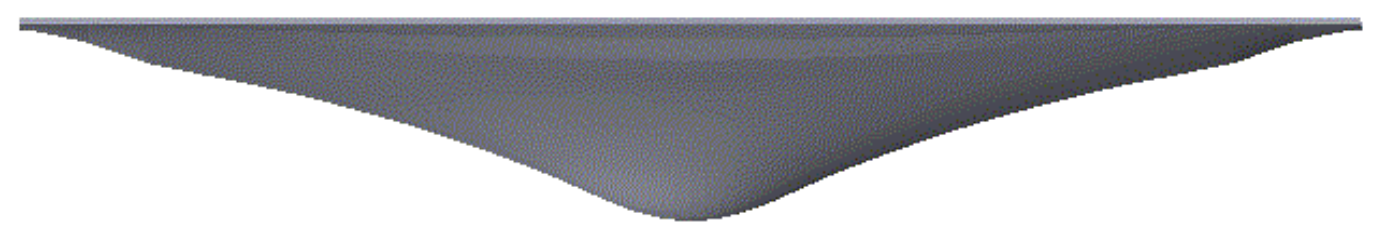

Figure 3.29 Residual deformation of a bare steel plate under blast loading modelled using shell elements in AUTODYN.

Figure 3.30 Residual deformation of a bare steel plate under blast loading modelled using hex elements in AUTODYN.

The curves shown in Figure 3.28 would have been slightly affected by the locations of the gauges, which sit at the centre of the elements rather than at the nodes. For the shell elements, the gauge was located $5.8 \mathrm{~mm}$ from the centre of the plate towards the outer corner, and in the plane of the shell element. For the hex elements, the gauge was located $19.3 \mathrm{~mm}$ from the centre of the plate towards the outer corner, and $1 \mathrm{~mm}$ from the centreline of the plate. Although this may have slightly affected the deformation histories in Figure 3.28, it does not account for the large difference in magnitude between the two curves. This is further evidenced by the residual deformations in Figure 3.29 and Figure 3.30, where the results had not been affected by the gauge location, yet a significant difference in deformation profile was observed.

Table 3.4 shows the numerical modelling results for the residual deformation at the plate centre compared with the experimental results for the bare steel plates. Validation experiment V3 was simulated in the same manner as V1 and V2, but with the material model for D36 used for the steel plate. The models using shell elements 
showed close agreement with the experiments, whereas the models using hex elements under-predicted the residual deformation by over $40 \%$. Note that test V4 was not modelled as fracture is not being investigated in this study. All these results take into account the plate thickness and position of the gauge.

Table 3.4 Numerical modelling results for the deformation of the plate centre compared with experimental results.

\begin{tabular}{llllllll}
\hline & & Charge & Experimental & \multicolumn{2}{c}{$\begin{array}{l}\text { Numerical bulge } \\
\text { depth }(\mathrm{mm})\end{array}$} & $\begin{array}{l}\text { Percentage error } \\
(\%)\end{array}$ & \\
Test & $\begin{array}{l}\text { Polyurea } \\
\text { coating }\end{array}$ & $\begin{array}{l}\text { Stand-off } \\
(\mathrm{mm})\end{array}$ & $\begin{array}{l}\text { bulge depth } \\
(\mathrm{mm})\end{array}$ & Shell & Hex & Shell & Hex \\
\hline V1 & None & 77.5 & 120 & 122 & 69 & 1.6 & 43 \\
V2 & None & 77.5 & 120 & 122 & 69 & 1.6 & 43 \\
V3 & None & 60.5 & 143 & 141 & 79 & 1.4 & 44 \\
\hline
\end{tabular}

The final plate deformation profiles for test V1, calculated using AUTODYN, are compared to the experimental deformation contours in Figure 3.31. The contours in the figure are all taken along the back surface (opposite to the blast) of the plate, moving from the centre of the plate to the centre of one of the edges. It can be seen that the numerical model using shell elements resulted in very good agreement with the experimental result for both the peak residual plate deformation and the plate profile. Conversely, the numerical model using hex elements did not give an accurate result for the peak residual plate deformation. However, the profile of the deformed plate using hex elements was generally consistent with the shape of the experimental plate profile. The results show that using shell elements allows the deformation to be faithfully accounted for by bending and membrane stretching, without the requirement to model the through-thickness stress wave propagation. 


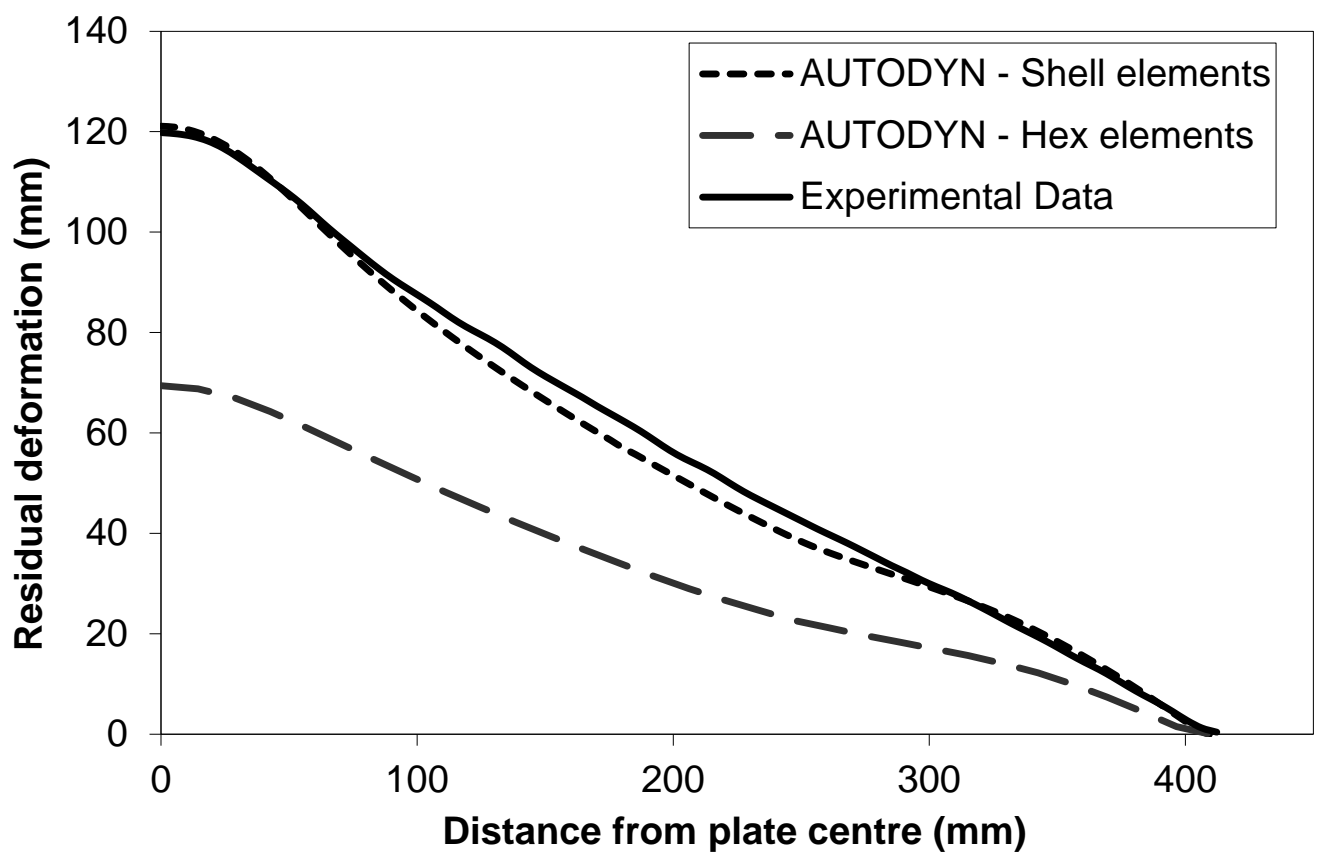

Figure 3.31 Comparison of numerically calculated residual deformation profiles with the experimental result (test V1).

Another measure used to determine the accuracy of the model was to compare the deformation history in the numerical model with the deformation-time points measured during test V1, shown in Figure 3.32. The results match very well during the very early stages of deformation, indicating that both of the models predict the initial plate acceleration with high accuracy. At a time of approximately $0.1 \mathrm{~ms}$ postdetonation, the AUTODYN model using hex elements decelerates more rapidly than the experimental deformation, resulting in under-predicted deformations. The AUTODYN model using shell elements matches the experimental deformation results closely, aside from the peak transient deformation, which was between $131 \mathrm{~mm}$ and $143 \mathrm{~mm}$ in the experiment and $122 \mathrm{~mm}$ in the numerical model. The use of hex elements appears to provide predictions of greater plate springback, which is the difference between the peak (transient) and residual deformation. However as the calculated residual deformation using hex elements $(69 \mathrm{~mm})$ was close to the peak deformation (71 mm), the springback was only calculated to be $2 \mathrm{~mm}$. The springback using the shell elements was calculated to be only $1 \mathrm{~mm}$. In comparison, the experimental springback was calculated to be at least $8 \mathrm{~mm}$. An improved 
experimental method for measuring peak transient deformation would be required to determine the extent of this disagreement between experiment and model.

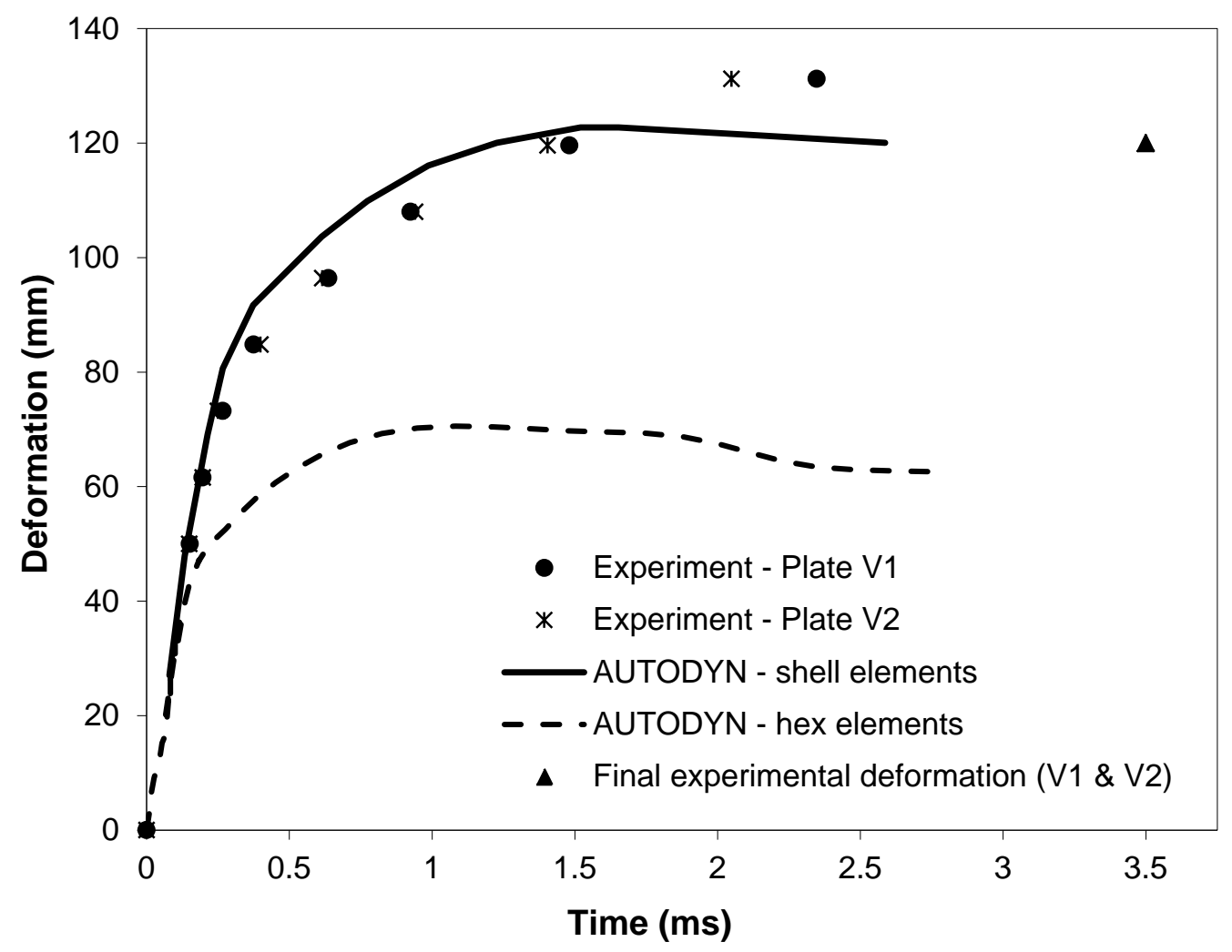

Figure 3.32 Comparison between AUTODYN and experimental deformations for test V1.

It should be noted that there was no experimental data available to validate the actual load on the plate. Conducting a mesh refinement study, in combination with the use of the most appropriate material model for the explosive, was deemed to give the best estimation of the load on the plate. Creating a numerical model to compare with the experimental pressure gauge measurements was considered; however this would not validate the load on the plate surface for a number of reasons. Firstly, the pressure measurements are made in a blast load regime where air-shock effects dominate, whereas the load on the plate in the detonics regime is dominated by the flow of the detonation products. Secondly, the element size identified in the mesh refinement studies that would be required to accurately model the detonation means that extending the model to 5 or $10 \mathrm{~m}$ from the charge would require too many elements 
for the capability of the computer. Thirdly, the JWL material model used for pentolite does not predict far-field pressures well, as it is based on empirical data of the explosive acceleration of metal casings. It is, however, accurate in predicting the acceleration of metal at very close range, which gives confidence in its use in the detonics regime-based numerical studies presented here.

\subsection{Numerical simulation and comparison with the experimental results: polyurea-coated plates}

The setup that was used to model the polyurea-coated steel plates is shown in Figure 3.33. The stand-off between the charge and the plate was adjusted from the bare steel plate model such that the centre of the charge was $61.5 \mathrm{~mm}$ above the surface of the plate, to be consistent with the experiment. The $410 \mathrm{~mm} \times 410 \mathrm{~mm}$ quarter plate was modelled using $5 \mathrm{~mm} \times 5 \mathrm{~mm}$ shell elements.

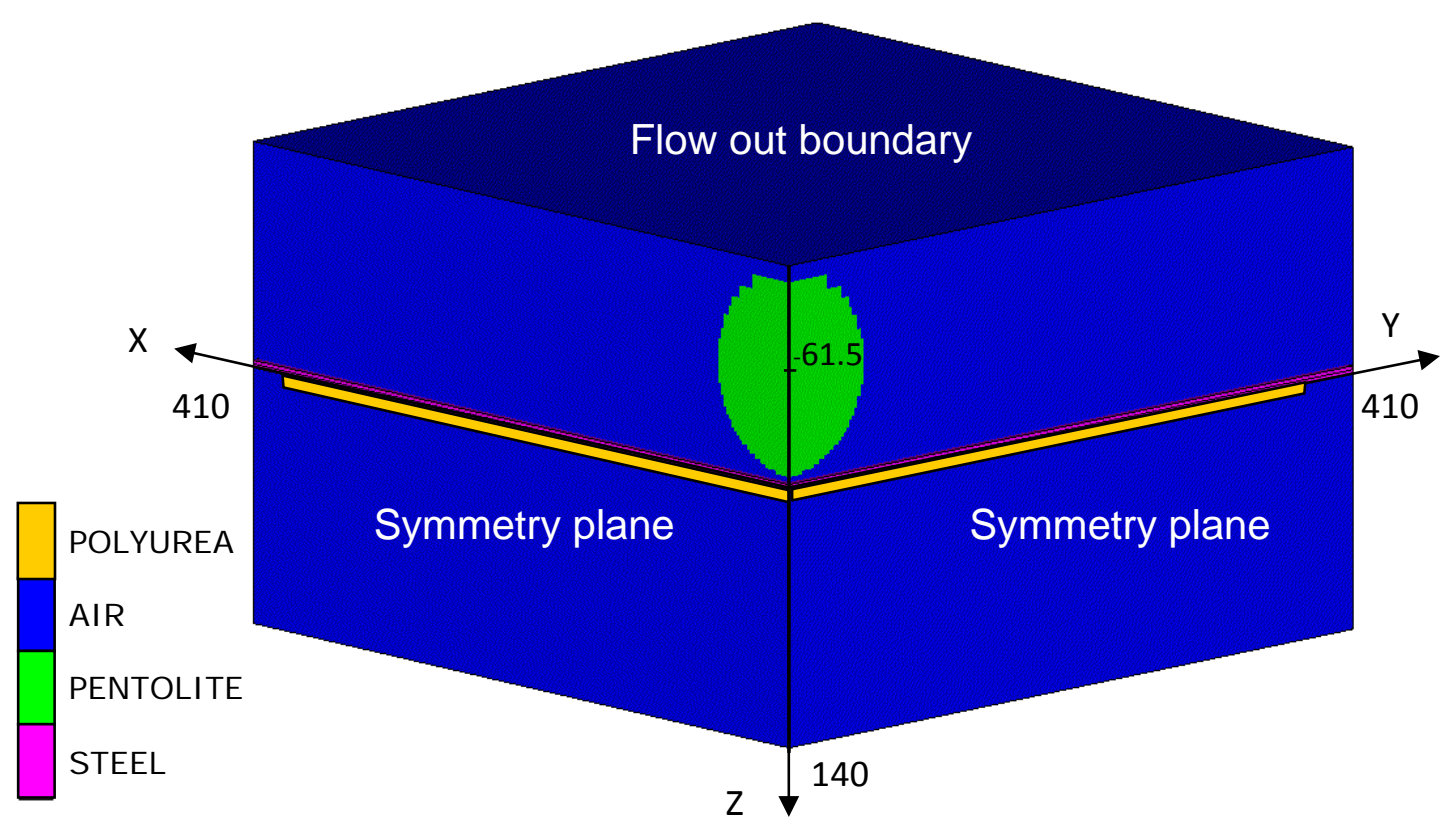

Figure 3.33 Setup for model of a polyurea-coated steel plate in AUTODYN.

Due to the large number of elements required to model the air-blast loading accurately, the number of elements used to model the polyurea was limited. The width and length of the elements were set to $5 \mathrm{~mm} \times 5 \mathrm{~mm}$, with five elements used through the thickness of the $10 \mathrm{~mm}$ coating and ten through the thickness of the $19 \mathrm{~mm}$ 
coating. Referring to Figure 3.21, elements of this thickness may result in a slight over-prediction of the plate deformation, however this is considered to be within an acceptable error range given the computational limitations. The polyurea coating extended over an area of $385 \times 385 \mathrm{~mm}$ to match the experiment, corresponding to 77 elements along the edge of the polyurea.

Again, the air was removed from the model after $3 \mathrm{~ms}$ when the loading of the plate was complete and a static damping constant of $5.5 \times 10^{-4}$ was applied to allow the solution to converge.

Table 3.5 shows the numerical modelling results for the residual deformation of the plate centre compared with the experimental results reported by Ackland et al. (2007). Figure 3.34 shows contour lines generated by the model overlaid on photographs of the plates to give a qualitative comparison.

Table 3.5 Numerical modelling results of polyurea-coated D36 steel plate results compared to experimental from Ackland et al. (2007).

\begin{tabular}{llllll}
\hline Test & $\begin{array}{l}\text { Polyurea } \\
\text { coating } \\
\text { thickness } \\
(\mathrm{mm})\end{array}$ & $\begin{array}{l}\text { Charge } \\
\text { stand-off } \\
(\mathrm{mm})\end{array}$ & $\begin{array}{l}\text { Experimental } \\
\text { bulge depth } \\
(\mathrm{mm})\end{array}$ & $\begin{array}{l}\text { Numerical } \\
\text { bulge depth } \\
(\mathrm{mm})\end{array}$ & $\begin{array}{l}\text { Percentage } \\
\text { error (\%) }\end{array}$ \\
\hline V5 & 10 & 61.5 & 134 & 122 & 9 \\
V6 & 19 & 61.5 & 109 & 111 & 2 \\
\hline
\end{tabular}




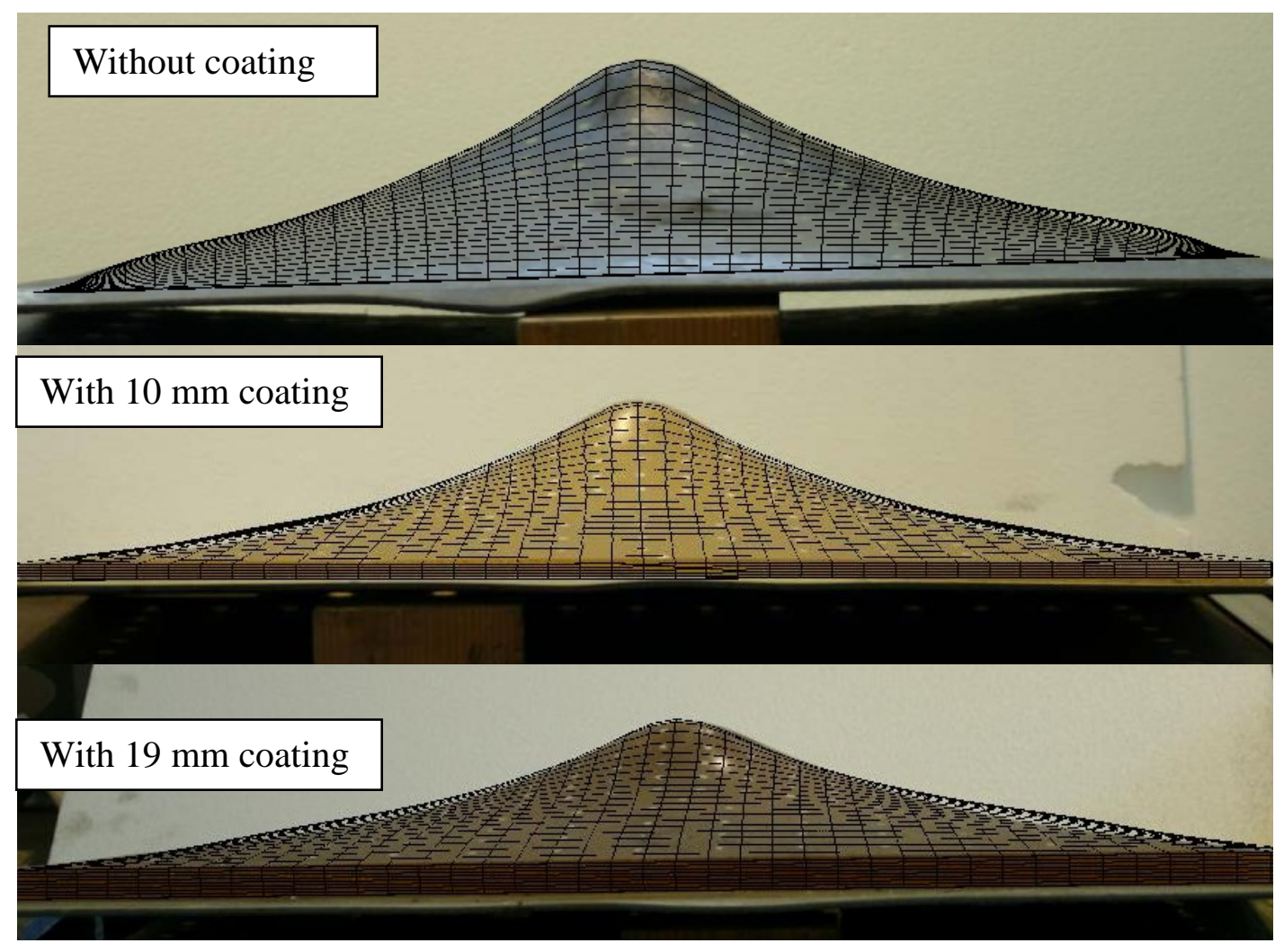

Figure 3.34 Photographs of the uncoated plate V3 (top), the plate with $10 \mathrm{~mm}$ thick coating V5 (middle), and the plate with $19 \mathrm{~mm}$ coating V6 (bottom), showing contour lines from numerical models overlaid on experimental targets.

Close agreement was found between the numerical models and the experiments for the maximum deformation and the deformation profile. Slight variation in the results can be seen near the plate boundaries in Figure 3.34, with the numerical models showing greater deformations near the boundaries compared with the experiments. It is believed that this may be due to the difference in the boundary conditions between the models and the experiments. The numerical models used fixed boundaries whereas in the experiments the plates were bolted to the test rig, allowing potential for plate slippage and elongation.

\subsection{Summary}

In this chapter, experimental and numerical techniques were developed to allow further investigation into the behaviour of polyurea-coated plates under blast loading. 
A method for measuring the transient deformation was also developed in the experimental testing. The main disadvantage of the method used is that it only measures deformation at discrete intervals, meaning that the peak transient deformation was not accurately captured. The measurement of peak transient deformation is improved with the use of high-speed photographic techniques in the next set of experiments (Chapter 4).

Numerical models in AUTODYN were developed to model the deformation of bare steel and polyurea-coated plates under blast loading, and validated against experimental results. This included extensive mesh sensitivity studies to effectively capture the loading and plate response. Two element types were investigated to model the plates, and 4-noded shell elements were found to better predict the plate response than 8-noded hex elements. It is believed this could be due to shear locking of the hex elements, which would explain the significant under-prediction of plate deformation with such elements. The numerical models were shown to have excellent agreement with the experimental results, and as such provide a validated basis for the numerical modelling which is presented in Chapter 5. 


\section{Chapter 4}

\section{Explosive Testing of Polyurea-Coated Steel Plates}

\subsection{Introduction}

This chapter presents the results of explosive blast tests to assess the effect of applying polyurea coatings to steel plates under close-in, localised blast loading. More specifically, the tests were performed to evaluate whether polyurea-coated steel plates would perform better under blast loading than bare steel plates of equivalent areal density. In addition, two different polyurea coating thicknesses were tested so that the effect of the coating to steel mass ratio on the blast response could be studied. The experiments were also designed with improved measurement techniques to better capture the peak transient deformation compared to the technique used in Chapter 3. As part of this, a new test rig was designed and built with increased strength and a number of instrumentation ports. The results of the experiments also provide valuable experimental data, which is used in Chapter 5 to further develop and validate numerical models.

\subsection{Blast rig design}

A new blast test rig was designed and manufactured for the experimental testing of polyurea-coated steel plates, shown in Figure 4.1. The rig was manufactured by the Department of Defence Prototype Engineering Centre (PEC), Laverton, to the design provided in Appendix A. 


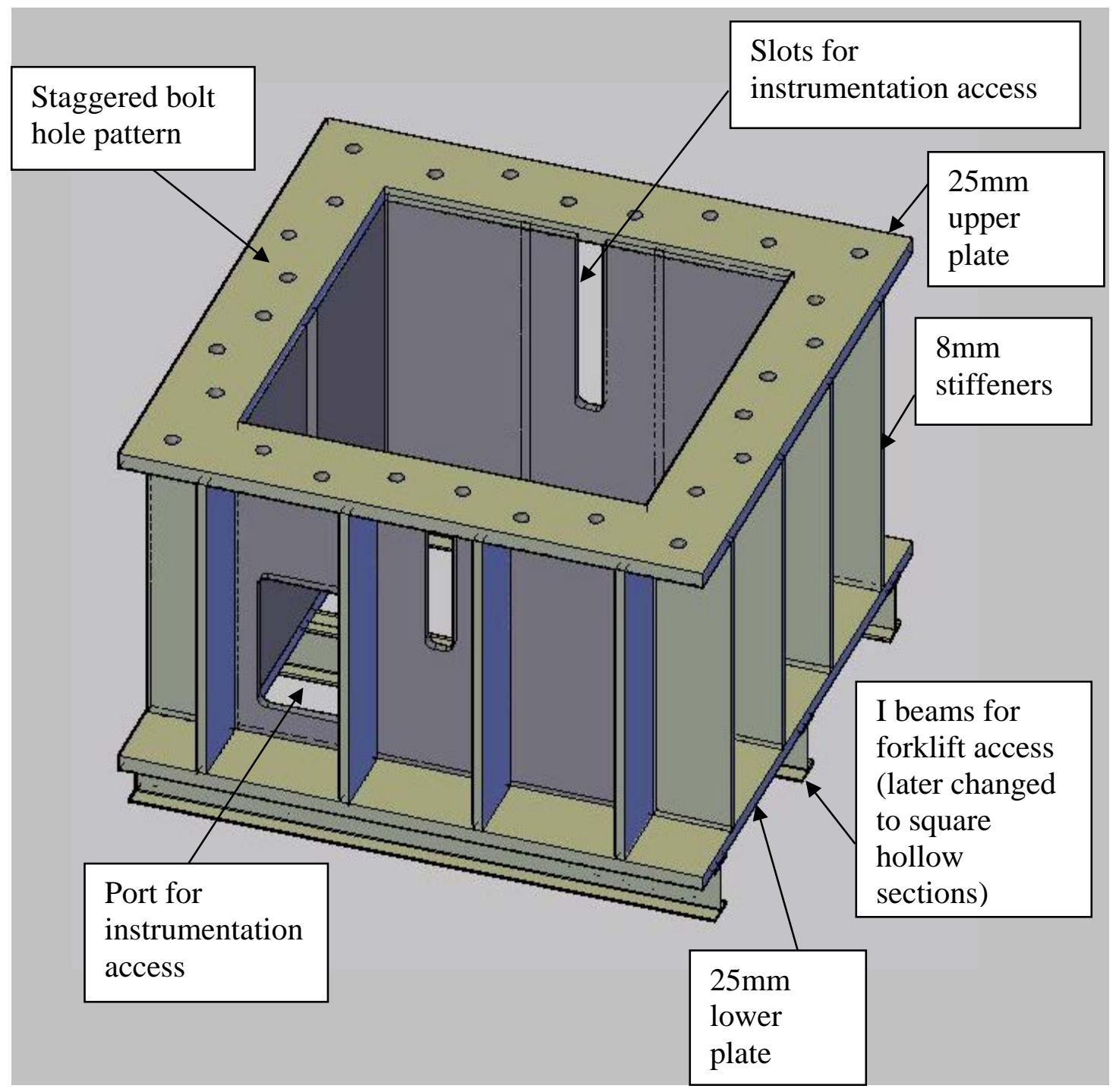

Figure 4.1 New blast test rig (not showing top plate or bolts). The total mass of the test rig including the top plate and bolts was approximately $640 \mathrm{~kg}$.

The steel plates used on the bolting surface were $25 \mathrm{~mm}$ thick, while $8 \mathrm{~mm}$ thick steel was used for the rest of the rig. During testing, a cover plate was also bolted to the top of the test plate to reduce slippage and to make the boundary conditions as close to a clamped boundary as possible. The unclamped test area was $700 \mathrm{~mm} \times 700 \mathrm{~mm}$. The bolt holes were staggered to spread the clamping load over the clamping surface, and the clamping surfaces were machined flat to ensure a uniform contact with the test plates.

The test rig was designed with six ports to allow instrumentation to be mounted onto the rig. A high speed camera, laser displacement transducer, a temperature 
thermocouple and a collapsible mechanical probe were all incorporated into the experimental setup for this series of tests. The instruments are explained in detail later in this chapter.

\subsection{Test plates}

Bluescope XLERPLATE 350 grade steel was chosen as the base plate material due to its price and availability, and because it had been used previously in validation experiments, i.e. tests V1 and V2, and in preliminary modelling (see Section 3.2). The plate thicknesses were chosen to use readily available thicknesses of steel. A polyurea areal density of $4.7 \mathrm{~g} / \mathrm{cm}^{2}$ was selected based on a $6 \mathrm{~mm}$ bare steel plate, and coatings were applied to $4 \mathrm{~mm}$ and $5 \mathrm{~mm}$ plates to give the same areal density over the test section.

Test plates were cut and drilled by PEC Laverton based on the engineering drawings in Appendix A. The plates were $1 \mathrm{~m} \times 1 \mathrm{~m}$ and had bolt holes drilled using a $27 \mathrm{~mm}$ AustBroach high speed steel (HSS) cutter to match the bolt holes of the blast rig. Ten plates were manufactured, of which six plates had polymer coatings applied to one surface.

The plates to which coatings were applied were first grit blasted and primed. The polyurea coatings were then cast onto the plates, covering an area of $670 \mathrm{~mm}$ $\times 670 \mathrm{~mm}$, which was slightly smaller than the test area $(700 \mathrm{~mm} \times 700 \mathrm{~mm})$, to allow the coatings to sit inside the test rig, shown in Figure 4.2. As in the validation experiments, polyurea was not applied over the clamping area to ensure that the boundary conditions were similar between the events. The polyurea coatings were made by DSTO using Versalink ${ }^{\circledR}$ P1000 Oligomeric Diamine and Isonate 143L. The mix ratio used was 100 parts Versalink P1000 and 26.4 parts Isonate 143L which gives a stoichiometry of $105 \%$ (excess isocyanate). The coating was tack free after 30 minutes, and while it was still in a soft state the edge barriers of the cast were removed and the edges were trimmed as necessary. The coating was then allowed to cure at room temperature for a minimum of $24 \mathrm{hrs}$ and then post-cured at $50^{\circ} \mathrm{C}$ for 24 hrs in an oven. All coatings were prepared over a week prior to testing. 


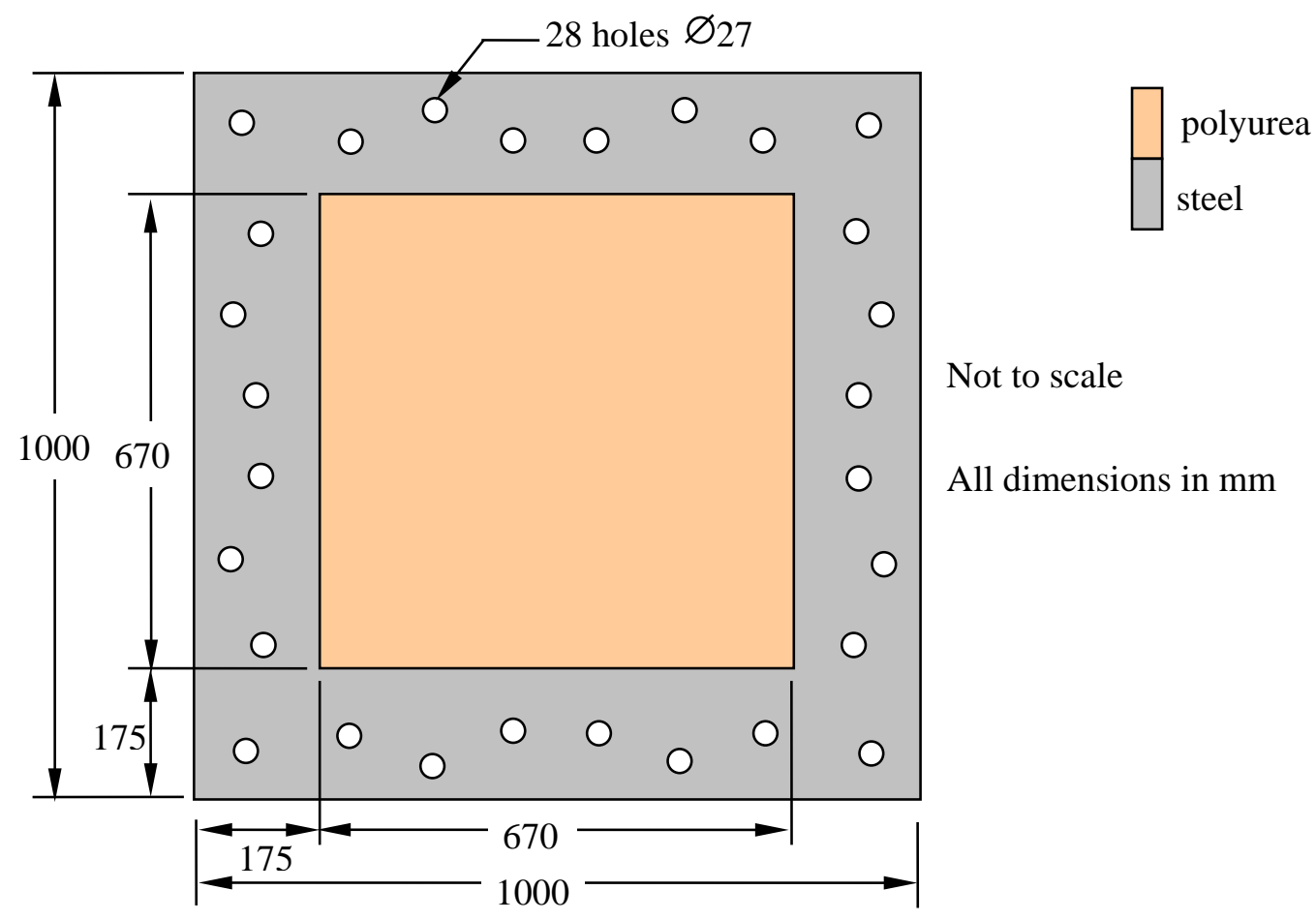

Figure 4.2 The polyurea coatings were applied over an area of $670 \mathrm{~mm} \times 670 \mathrm{~mm}$ to fit within the blast rig, which had a free-span area of $700 \mathrm{~mm} \times 700 \mathrm{~mm}$.

The coatings were applied on a mass basis rather than thickness to ensure that the areal density of all the plates were the same over the coated area. Table 4.1 shows details of the plate configurations. There was a slight discrepancy in thickness between the coatings, as the coating density of the thicker coating was expected to be double that of the thinner coating. Instead, the thick coating was $2 \%$ thicker than would be expected based on the thin coating measurement. This is likely to be due to method of measuring the thickness, which took an average from number of discrete points around the edge of the coating. This resulted in some uncertainty in the coating density, which was calculated to be $1.003 \pm 0.010 \mathrm{~g} / \mathrm{cm}^{3}$. 
Table 4.1 Test plate details. All test plates had an areal density of $4.7 \mathrm{~g} / \mathrm{cm}^{2}$.

\begin{tabular}{llll}
\hline Plate Numbers & Target description & $\begin{array}{l}\text { Coating } \\
\text { mass }(\mathrm{kg})\end{array}$ & $\begin{array}{l}\text { Coating } \\
\text { thickness } \\
(\mathrm{mm})\end{array}$ \\
\hline B1, B2, B3, B4 & 6mm bare steel & 0 & 0 \\
C1, C2, C3 & $5 \mathrm{~mm}$ steel + coating & 3.5 & 7.7 \\
D1, D2, D3 & 4mm steel + coating & 7.0 & 15.7 \\
\hline
\end{tabular}

\subsection{Determination of charge stand-off}

The preliminary numerical models from Chapter 3 were modified to match the new plate geometry and re-run to determine a stand-off distance that would produce a large amount of plate deformation using a 500 g spherical pentolite charge, whilst avoiding plate rupture. Based on the results, a stand-off gap of $10 \mathrm{~mm}$ between bottom of the the charge and the plate was chosen.

Using a stand-off gap of $10 \mathrm{~mm}$ is also advantageous when considering the numerical modelling of the charge detonation. In the literature review, it was identified that the JWL material model for explosives may lose accuracy when the explosive expands beyond two times its original volume. For a spherical $500 \mathrm{~g}$ pentolite charge, this equates to an expanded radius of $52.3 \mathrm{~mm}$, or a stand-off distance of $10.8 \mathrm{~mm}$. This means that in the numerical simulations of the experiments (which will be discussed in the next chapter), the loading at the plate centre should be simulated with good accuracy. The accuracy may drop off closer to the plate edges, where the expansion has increased well beyond this limit, however it is the loading near the plate centre which will most affect the plate deformation. 


\subsection{Experimental setup}

Experiments were performed at the Department of Defence's Proof and Experimental Establishment (P\&EE) Graytown during November 2009. Figure 4.3 shows the experimental setup.

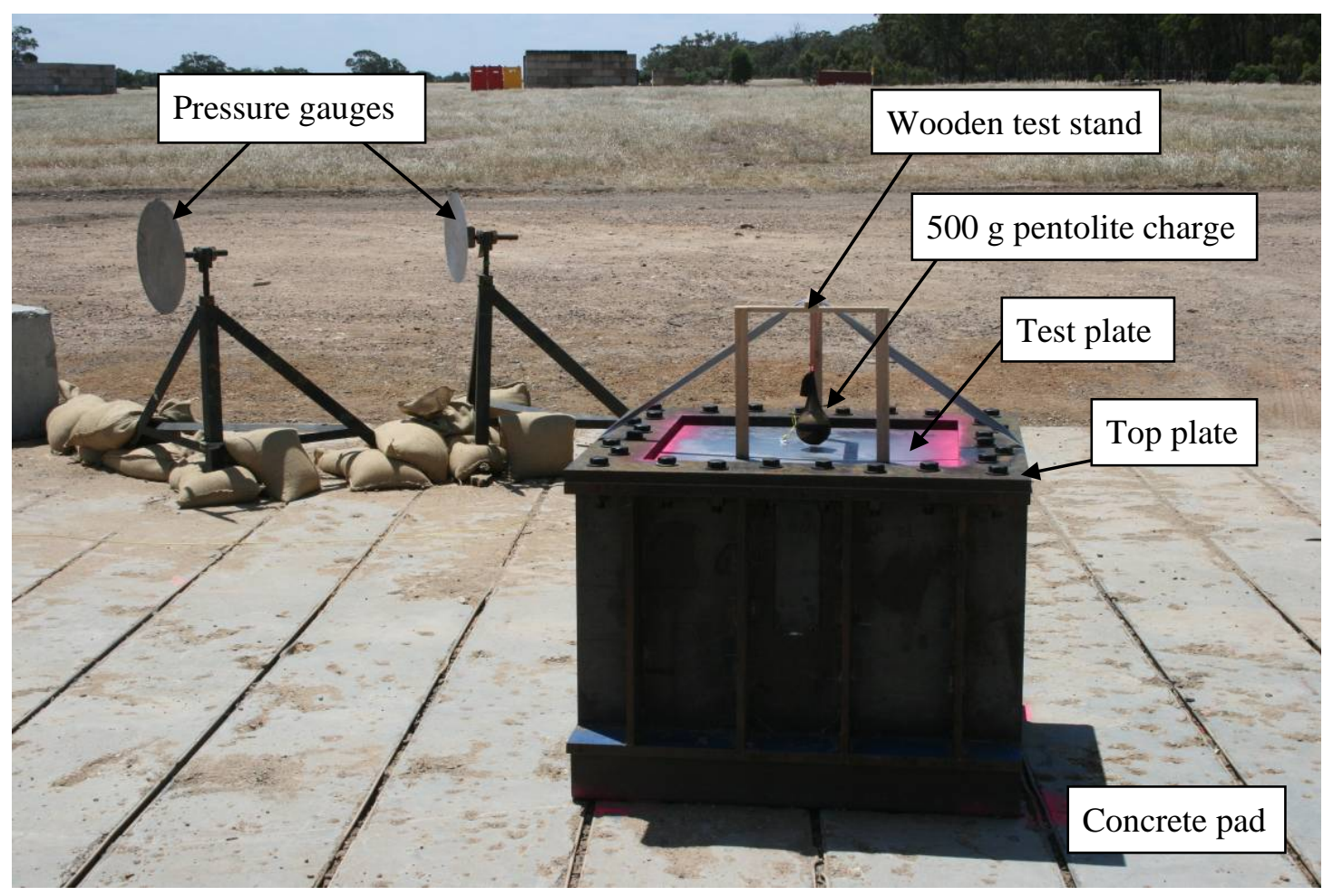

Figure 4.3 Pre-event experimental setup.

The blast tests were conducted using nominally $500 \mathrm{~g}$ pentolite spheres. The charges were suspended in a stocking from a wooden frame to ensure minimal interference with the formation of the blast wave. The charges were detonated using RP501 exploding bridgewire (EBW) detonators located centrally within the charge. The actual mass of each charge was recorded prior to the event and these are presented later, in the experimental results section. The plates were bolted to the test rig using a torque wrench to achieve a torque of $160 \mathrm{Nm}$ on each of the bolts.

Three tests of each plate configuration were conducted to give an indication of the variability in the experimental results. Due to financial and time limitations, more repeat tests were not conducted. Although a set of three tests is not sufficient for full 
statistical significance, it is enough to give a good indication of any variability between the experiments.

\subsubsection{Instrumentation overview}

A number of recording devices were employed to capture data during the event, many for the first time in such a scenario. Table 4.2 summarises which instrumentation was used for each event. The first three events were un-instrumented because there was considered to be a risk that the plate would rupture and cause damage to instrumentation within the blast rig. Instrumentation was used on later tests when it was evident that rupture did not occur. 
Chapter 4. Explosive Testing of Polyurea-Coated Steel Plates.

Table 4.2 Instrumentation summary. The ticks indicate devices which were used and the crosses indicate devices which weren’t used.

\begin{tabular}{|c|c|c|c|c|c|}
\hline Event & Plate & $\begin{array}{l}\text { Pressure } \\
\text { Gauge }\end{array}$ & $\begin{array}{l}\text { Collapsible } \\
\text { probe }\end{array}$ & $\begin{array}{l}\text { Miro-3 high } \\
\text { speed camera }\end{array}$ & $\begin{array}{l}\text { Laser displacement } \\
\text { transducer }\end{array}$ \\
\hline 1 & B1 & $\checkmark$ & $x$ & $x$ & $x$ \\
\hline 2 & D1 & $\checkmark$ & $x$ & $x$ & $x$ \\
\hline 3 & C1 & $\checkmark$ & $x$ & $x$ & $x$ \\
\hline 4 & B2 & $\checkmark$ & $x$ & $x$ & $\checkmark$ \\
\hline 5 & D2 & $\checkmark$ & $x$ & $x$ & $\checkmark$ \\
\hline 6 & C2 & $\checkmark$ & $x$ & $x$ & $\checkmark$ \\
\hline 7 & B3 & $\checkmark$ & $\checkmark$ & $\checkmark$ & $\checkmark$ \\
\hline 8 & B4 & $\checkmark$ & $x$ & $\checkmark$ & $x$ \\
\hline 9 & C3 & $\checkmark$ & $x$ & $\checkmark$ & $x$ \\
\hline 10 & D3 & $\checkmark$ & $x$ & $\checkmark$ & $x$ \\
\hline
\end{tabular}

\subsubsection{Pressure measurement}

Two PCB ${ }^{\circledR}$ model 101A05 pressure gauges with a range of $689 \mathrm{kPa}(100 \mathrm{psi})$ and a $5.0 \mathrm{MHz}$ sampling rate were positioned at a distance of $4 \mathrm{~m}$ from the charge and a height of $1 \mathrm{~m}$ above the ground. The purpose of the gauges was to check the consistency of the blast waves during the events. The gauges were mounted using baffle plates to ensure minimal interference of the dynamic flow of the blast wave for the static pressure reading. After the second event the gauges were moved to a distance of $2 \mathrm{~m}$ from the charge as the pressures recorded at $4 \mathrm{~m}$ were well below the maximum range of the gauges. 


\subsubsection{Residual deformation measurement}

The residual deformation of each plate was measured after the event before the top plate was removed, as shown in Figure 4.4. This was achieved by placing a level ruler across the front of the plate and lowering a vernier caliper into the bulge at its deepest point.
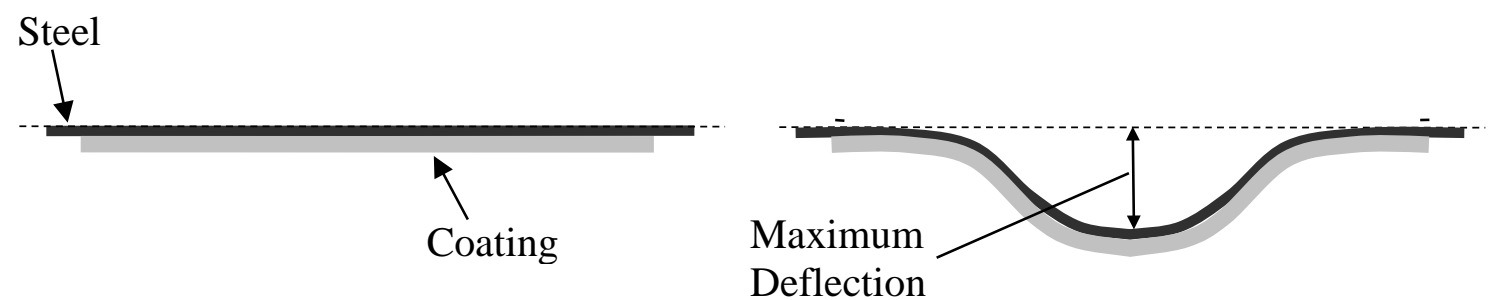

Figure 4.4 Flat specimen pre-test (left); Deformed specimen after testing (right).

\subsubsection{Transient deformation measurement}

Three methods were used to measure the transient deformation of the plates: high speed imagery; a laser displacement transducer; and a collapsible mechanical probe. The instruments are shown set up in the test rig in Figure 4.5. 


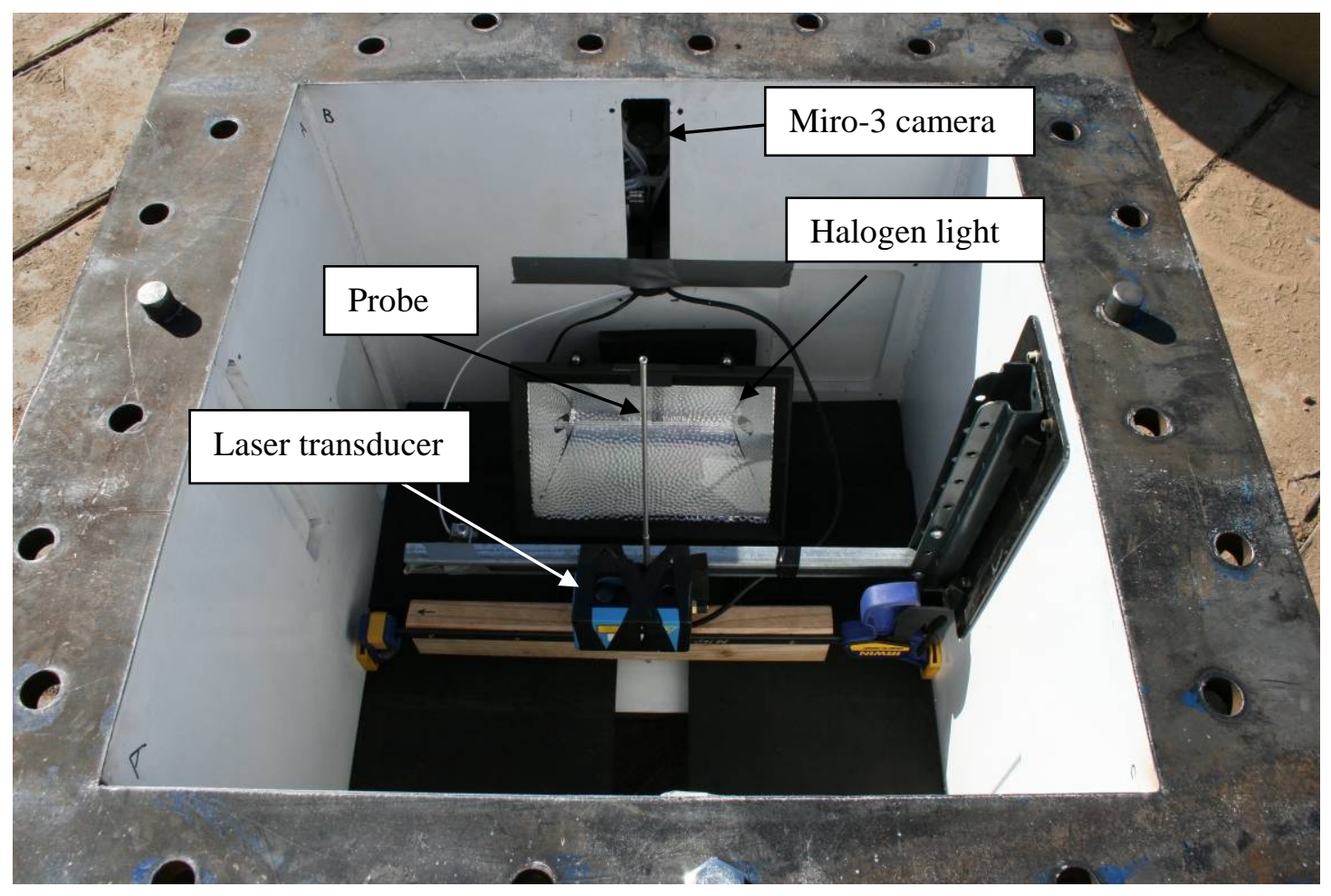

Figure 4.5 Blast rig internal setup showing the instrumentation for transient deformation measurement.

A Phantom Miro-3 high speed camera was mounted to the test rig to record the transient deformation of the plate centre. The camera was mounted within the test rig as it is small and rated to survive $100 \mathrm{~g}$ acceleration. The camera has a maximum resolution of $800 \times 600$ pixels, a maximum framing rate of 111,110 frames per second at a resolution of $32 \times 16$ pixels and a minimum shutter speed of $2 \mu$ s. The setup of the camera requires the operator to choose a resolution and frame rate combination to suit the speed and light conditions of the event. As the frame rate is increased, the resolution must be reduced.

To capture the movement of the plate centre, the camera was focussed on the plane in which the centre of the plate would move. This was achieved by positioning a scale in this plane and adjusting the focus accordingly. A pre-event still image was also taken of the scale to use as a reference when determining the location of the plate centre during and after the event, Figure 4.6. A resolution of $624 \times 112$ pixels was chosen to capture the entire range of the deformation of the plate centre whilst still allowing a sufficiently high framing rate. 


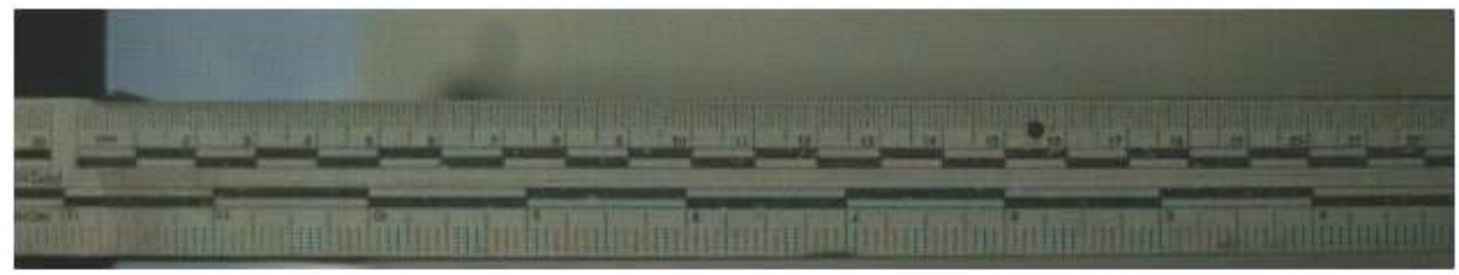

Figure 4.6 Still image of the scale taken using the high speed camera. The same resolution was used for the high speed video imaging $(624 \times 112$ pixels). The pixel size allowed a discretisation of $0.5 \mathrm{~mm}$ in the scale.

A shutter speed of $5 \mu$ s was set based on an estimated initial plate velocity of $200 \mathrm{~m} / \mathrm{s}$ (from preliminary numerical simulations) and a required resolution of $1 \mathrm{~mm}$ during the initial acceleration. A framing rate of 8113 frames per second was used, which is the maximum framing rate when recording at a resolution of $624 \times 112$ pixels.

In addition to the camera, a Micro-Epsilon OPTONCDT LD1627-200/5m/SGF laser displacement transducer was mounted inside the test rig to measure the transient deformation of the plate. The laser was a class 3R with an output power of $<=2.8 \mathrm{~mW}$ and a wavelength of $670 \mathrm{~nm}$. A sampling frequency of $100 \mathrm{kHz}$ was used.

A collapsible aluminium mechanical probe was installed in the rig during one of the events to measure the peak transient deformation of the plate. The probe can be seen in Figure 4.7 and is also shown in Figure 4.5. This method had been used successfully during other trials where the deforming plates push on the pre-extended probe and leave it in a compressed or partially collapsed state for the post event measurement. 


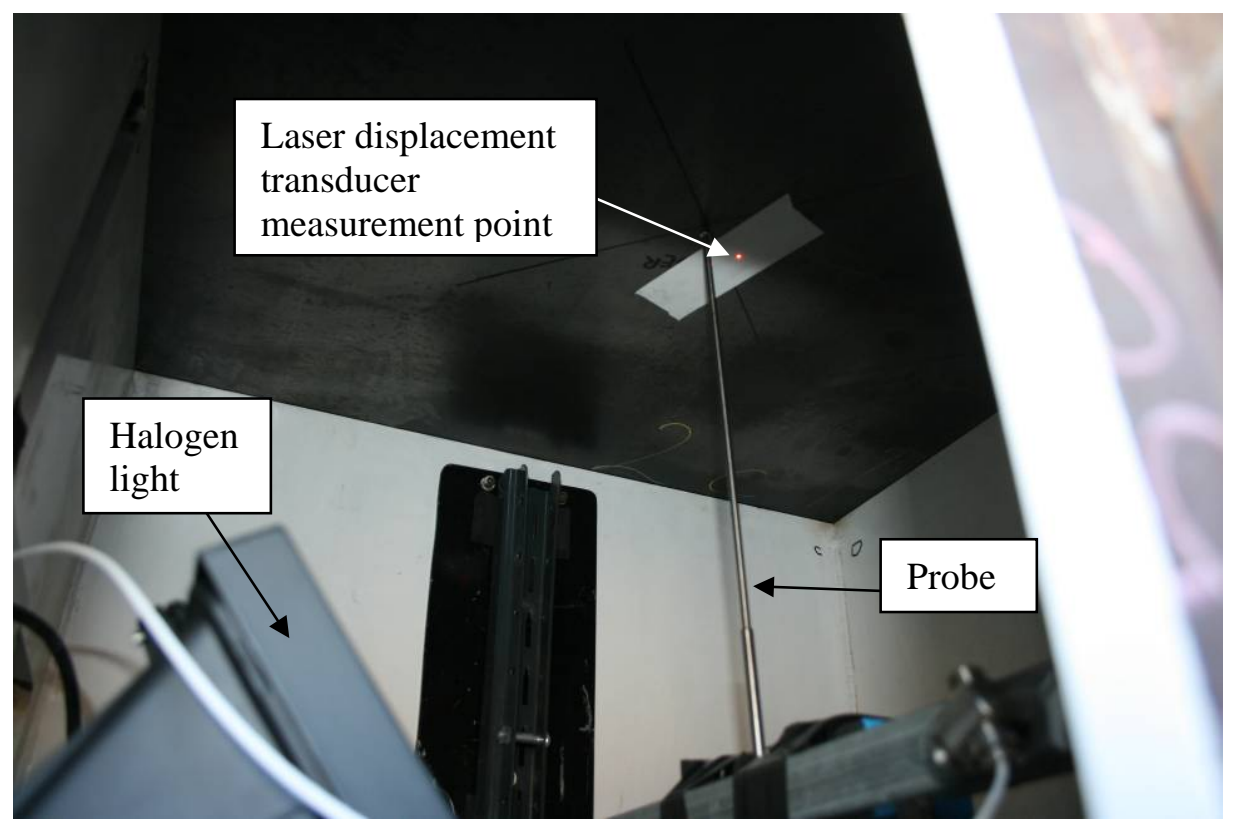

Figure 4.7 Setup inside the test rig showing the mechanical probe positioned with its tip against the plate centre.

\subsubsection{Temperature measurement}

The temperature of the plate surface was measured using a ThermoMETER LS handheld measurement device before each event. The emissivity value was set to 0.9 to match the emissivity of the steel plate.

\subsection{Experimental results}

\subsubsection{Validation of charge detonation}

The static pressure gauges recorded consistent signals for all events, indicating reliable detonations of the charges. Figure 4.8 shows an example of one of the pressure traces, recorded by pressure gauge P1 during Event 4. The incident shock front which has travelled directly from the explosive source to the gauge is observed as the first peak arriving at the gauge at around $2.1 \mathrm{~ms}$. A second spike is seen at around $4.9 \mathrm{~ms}$, which is a reflected shock off the ground. The full set of pressure gauge results can be found in Appendix C. 


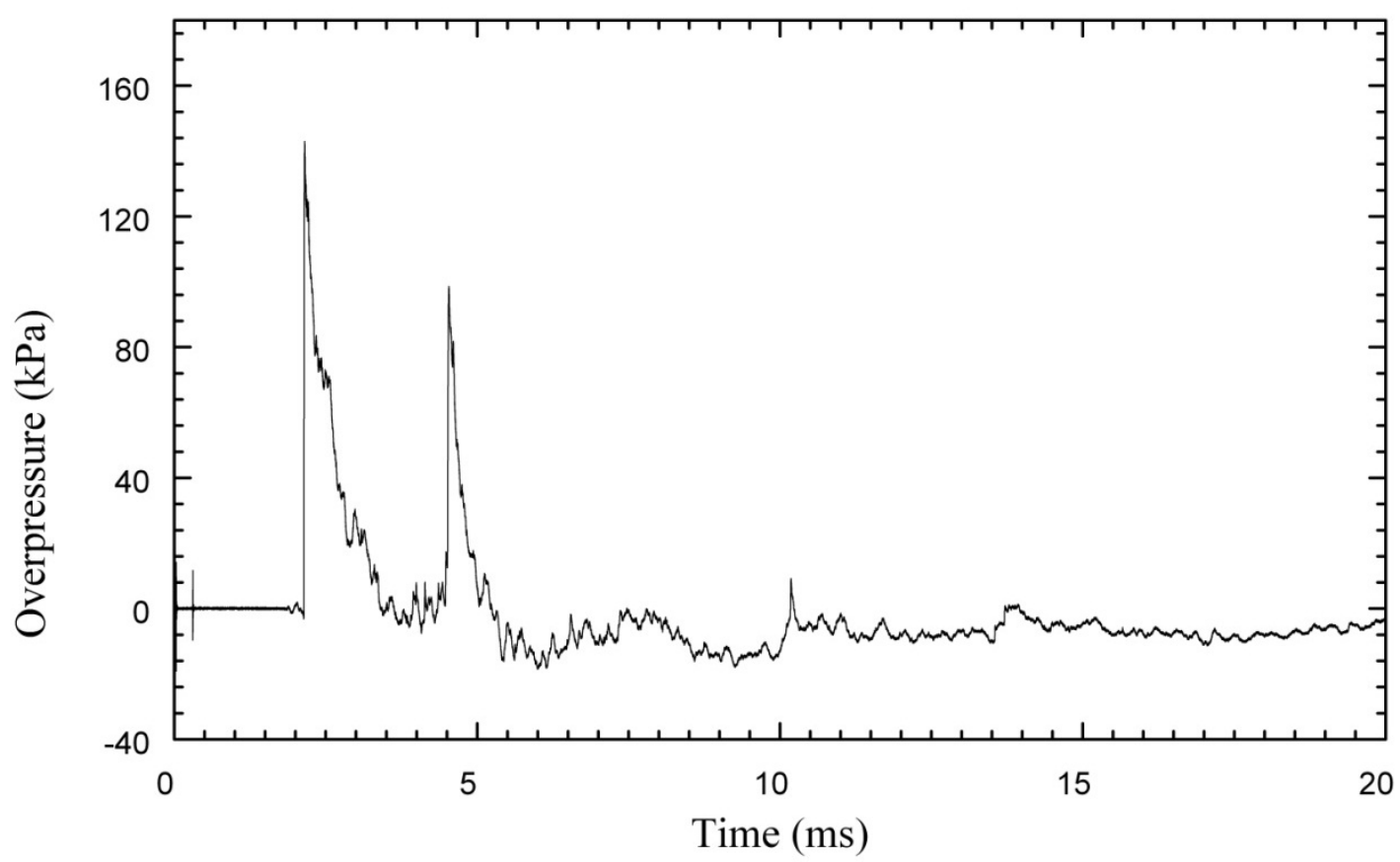

Figure 4.8 Pressure history recorded by gauge P1 at $4 \mathrm{~m}$ during Event 4.

\subsubsection{Residual deformation results}

The deformed plates were visually inspected after each event. Figure 4.9 shows plate B1 after Event 1. Figures 4.10 to 4.12 show a selection of the polyurea-coated plates, which all displayed signs of de-bonding. The thinner polyurea coatings on the $5 \mathrm{~mm}$ thick steel plates showed de-bonding over a circular area at the centre of the plate, for example Figure 4.10. The diameter of the de-bonded area was measured for each plate using a level ruler positioned across the centre of the plate and plumb bobs to locate the extent of the de-bonding. The diameters of the de-bonded area of the three plates were $480 \mathrm{~mm}, 505 \mathrm{~mm}$ and $500 \mathrm{~mm}$, for plates C1, C2 and C3 respectively, resulting in an average de-bonded area of $495 \mathrm{~mm}$ (radius $247.5 \mathrm{~mm}$ ). The thicker polyurea coatings on the $4 \mathrm{~mm}$ thick steel plates all de-bonded across a large area extending to the edges of the plate in all three cases, for example Figure 4.11. The coating on plate D2 (Event 5) tore around a circular area at the centre of the plate, as shown in Figure 4.12. This may have been initiated at an impurity (e.g. an air bubble) in the coating at that point, which could lead to failure in the coating as the region deformed. 
Chapter 4. Explosive Testing of Polyurea-Coated Steel Plates.

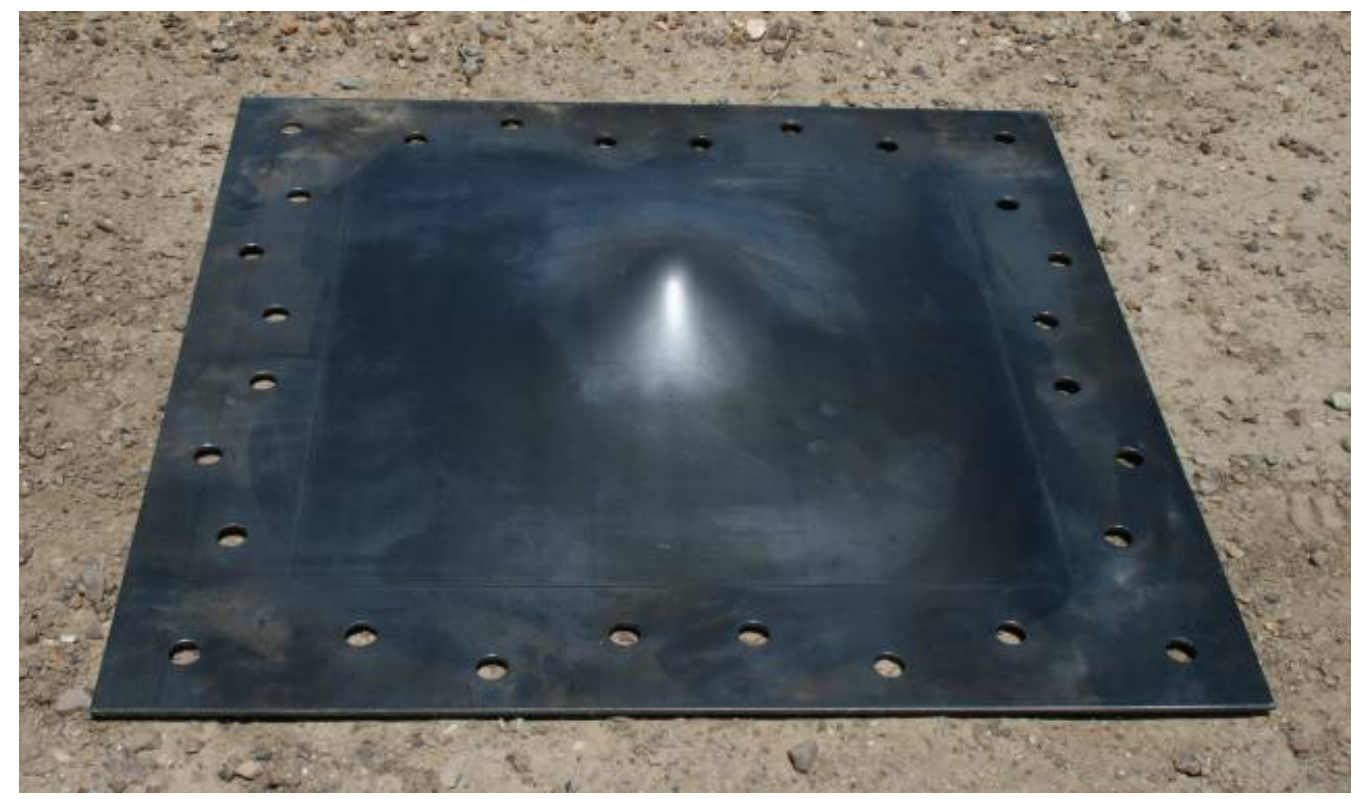

Figure 4.9 Post-event deformation of a bare $6 \mathrm{~mm}$ thick steel plate (plate B1).

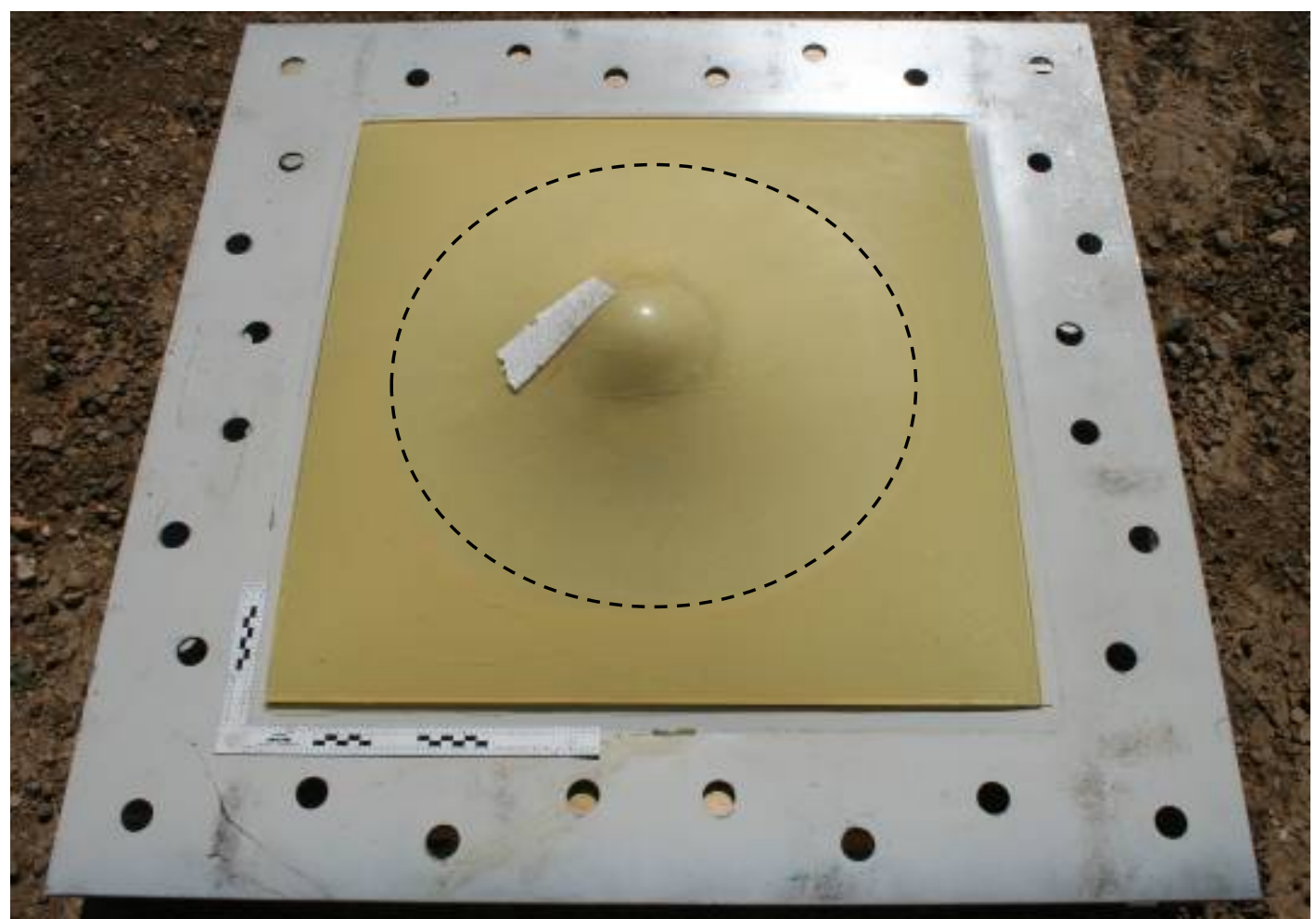

Figure 4.10 Post-event deformation of a $5 \mathrm{~mm}$ thick steel plate with a $7.7 \mathrm{~mm}$ polyurea coating (plate C3), showing de-bonding over a circular area at the centre of the plate (bordered by the dashed line). 


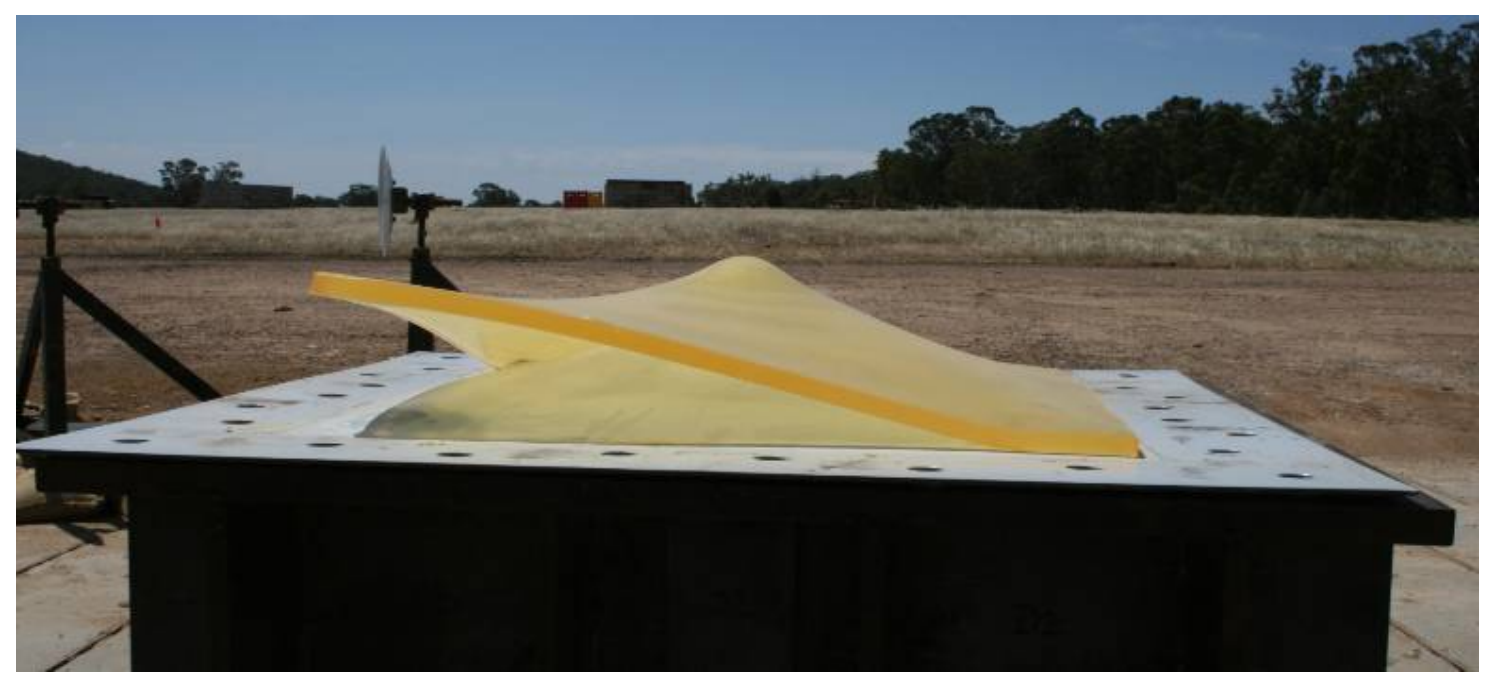

Figure 4.11 Post-event deformation of a $4 \mathrm{~mm}$ thick steel plate with a $15.7 \mathrm{~mm}$ thick polyurea coating (plate D1), showing de-bonding reaching the polyurea edge. The polyurea remained joined to the plate at the other three corners.

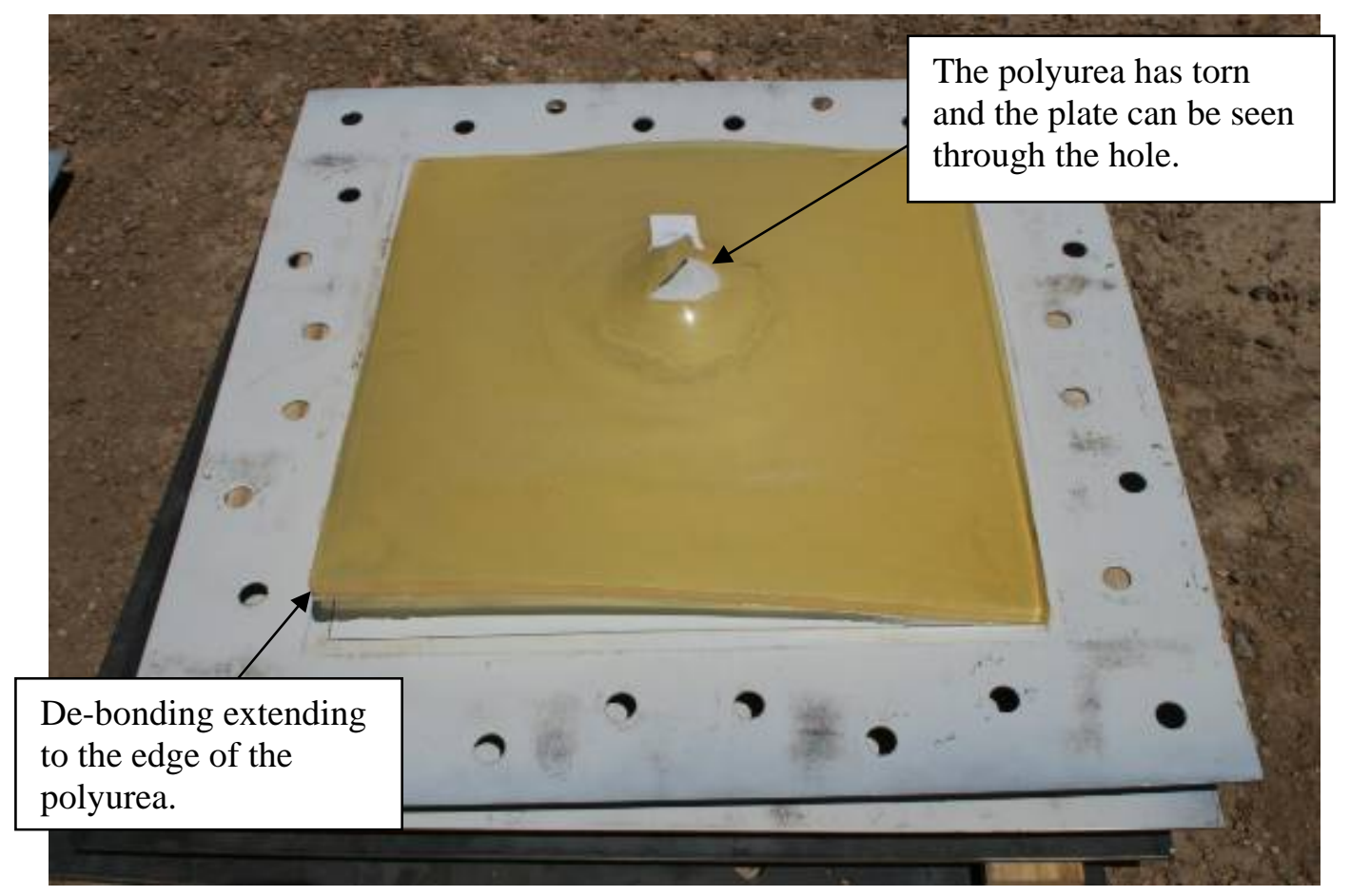

Figure 4.12 Post-event deformation of a $4 \mathrm{~mm}$ thick steel plates with a $15.7 \mathrm{~mm}$ thick polyurea coating (plate D2) showing tearing of the polyurea near the plate centre. Again, the polyurea remained joined to the plate at three of the corners. 
The plate (B3) ruptured during Event 7, as shown in Figure 4.13. The rupture was believed to be due to the use of the mechanical probe which initiated failure at the point of contact in the plate centre. This was evidenced by copper markings on the plate at the point of initiation and by the high speed camera results. It was believed that the probe initiated the plate rupture due to the large deformation of the plate compared to previous DSTO trials where this method had been used successfully. As a result, the mechanical probe was not used in any subsequent events.

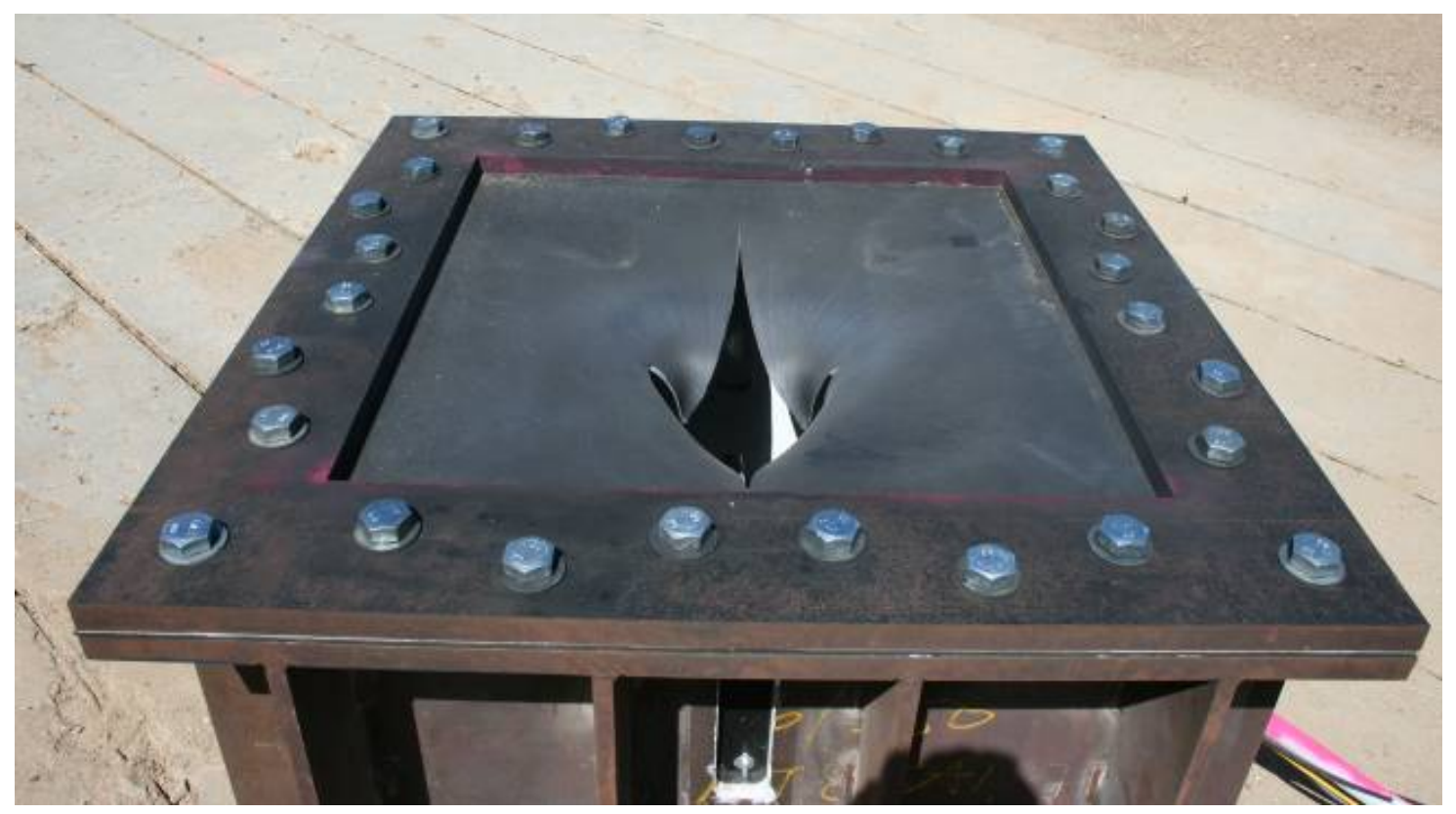

Figure 4.13 Bare $6 \mathrm{~mm}$ steel plate showing rupture after Event 7 (plate B3), in which a mechanical probe was positioned below the plate.

Table 4.3 and Table 4.4 show a summary of the measured results for peak residual deformation. The deformation profiles were also measured using a Konica Minolta Vivid 910 3D laser scanner after the plate had been removed from the test rig. The scanner was not used while the plate was attached to the rig as it was not portable, making it inappropriate for use in the field. The purpose of measuring the deformation profiles was to allow comparisons with numerical modelling. The peak deformations measured at the plate centre using the scanner were all within $2 \%$ of the deformations measured while the plate was attached to the rig. The discrepancies are believed to be mainly due to relaxation of the plate when it was removed from the rig. The profiles 
and deformations from the laser scanner are given in Appendix B (plate B3 profile with rupture not shown).

Table 4.3 Residual deformation results for the blast experiments. All the target plates had a total areal density of $4.7 \mathrm{~g} / \mathrm{cm}^{2}$ over the test area.

\begin{tabular}{|c|c|c|c|c|c|}
\hline $\begin{array}{l}\text { Event } \\
\text { Number }\end{array}$ & Plate & $\begin{array}{l}\text { Coating } \\
\text { Thickness* } \\
\text { (mm) }\end{array}$ & $\begin{array}{l}\text { Charge } \\
\text { Mass (g) }\end{array}$ & $\begin{array}{l}\text { Plate } \\
\text { Temperature } \\
\left({ }^{\circ} \mathrm{C}\right)\end{array}$ & $\begin{array}{l}\text { Plate } \\
\text { Deformation }^{\dagger} \\
(\mathrm{mm})\end{array}$ \\
\hline 1 & B1 & 0.0 & 494 & 43 & 120.4 \\
\hline 2 & D1 & 15.5 & 485 & 39 & 138.0 \\
\hline 3 & C1 & 8.0 & 491 & 40 & 125.1 \\
\hline 4 & B2 & 0.0 & 482 & 27 & 110.0 \\
\hline 5 & D2 & 15.7 & 490 & 36 & 133.6 \\
\hline 6 & $\mathrm{C} 2$ & 7.7 & 489 & 43 & 120.8 \\
\hline 7 & B3 & 0.0 & 483 & 32 & Rupture \\
\hline 8 & B4 & 0.0 & 487 & 50 & 115.0 \\
\hline 9 & C3 & 7.3 & 485 & 49 & 126.3 \\
\hline 10 & D3 & 16.1 & 490 & 37 & 135.7 \\
\hline
\end{tabular}

* Average coating thickness measured across the plate

\# Temperature measured before the event

${ }^{\dagger}$ Peak residual deformation at the plate centre measured while the plate was attached to the blast rig 
Chapter 4. Explosive Testing of Polyurea-Coated Steel Plates.

Table 4.4 Average residual deformation results for the blast experiments.

\begin{tabular}{llllll}
\hline $\begin{array}{l}\text { Target } \\
\text { description }\end{array}$ & $\begin{array}{l}\text { Average } \\
\text { coating } \\
\text { thickness } \\
(\mathrm{mm})\end{array}$ & $\begin{array}{l}\text { Average } \\
\text { charge mass } \\
(\mathrm{g})\end{array}$ & $\begin{array}{l}\text { Average } \\
\text { plate } \\
\text { temperature } \\
\left({ }^{\circ} \mathrm{C}\right)\end{array}$ & $\begin{array}{l}\text { Average } \\
\text { plate } \\
\text { deformation } \\
(\mathrm{mm})\end{array}$ & $\begin{array}{l}\text { Standard } \\
\text { deviation } \\
(\mathrm{mm})\end{array}$ \\
\hline $\begin{array}{l}6 \mathrm{~mm} \text { bare } \\
\text { steel }\end{array}$ & 0.0 & 487.7 & 40.0 & 115.1 & 5.2 \\
$\begin{array}{l}5 \text { mm steel }+ \\
\text { coating }\end{array}$ & 7.7 & 488.3 & 44.0 & 124.1 & 2.9 \\
4 mm steel + & 15.7 & & & & \\
coating & & 488.3 & 37.3 & 135.7 & 2.2 \\
\hline
\end{tabular}

\subsubsection{Effect of coating thickness on residual deformation}

Figure 4.14 shows a plot summarising the effect of coating thickness on peak residual plate deformation. For the plate with no polymer coating the three results show a spread of $\pm 5 \mathrm{~mm}$. This corresponds to a standard deviation of $5.2 \mathrm{~mm}$ and a percentage variation of $4.3 \%$, which is acceptable for this type of trial. The values recorded for the $7.7 \mathrm{~mm}$ thick polymer-coated plates show a tighter grouping, with a standard deviation of $2.9 \mathrm{~mm}$. The average residual deformation value for the $5 \mathrm{~mm}$ plates with $7.7 \mathrm{~mm}$ thick polyurea coatings is $9 \mathrm{~mm}$ larger than the average deformation of the mass-equivalent bare steel plate. The final grouping of results relates to a polymer coating thickness of $15.7 \mathrm{~mm}$ and has a standard deviation of $2.2 \mathrm{~mm}$. The average peak residual deformation of the $4 \mathrm{~mm}$ plates with $15.7 \mathrm{~mm}$ thick polyurea coatings is $20.6 \mathrm{~mm}$ greater than the bare steel plates.

The basic trend in the data here is that the residual deformation increases as the polymer coating thickness (or the polymer to steel ratio) is increased. This suggests that the use of polymer coatings reduces the resistance of the plates to blast loading when considering residual deformations alone. However in practical application, the 
transient deformations are more important than the residual deformation, as they define the area behind which damage to objects can occur during the blast event.

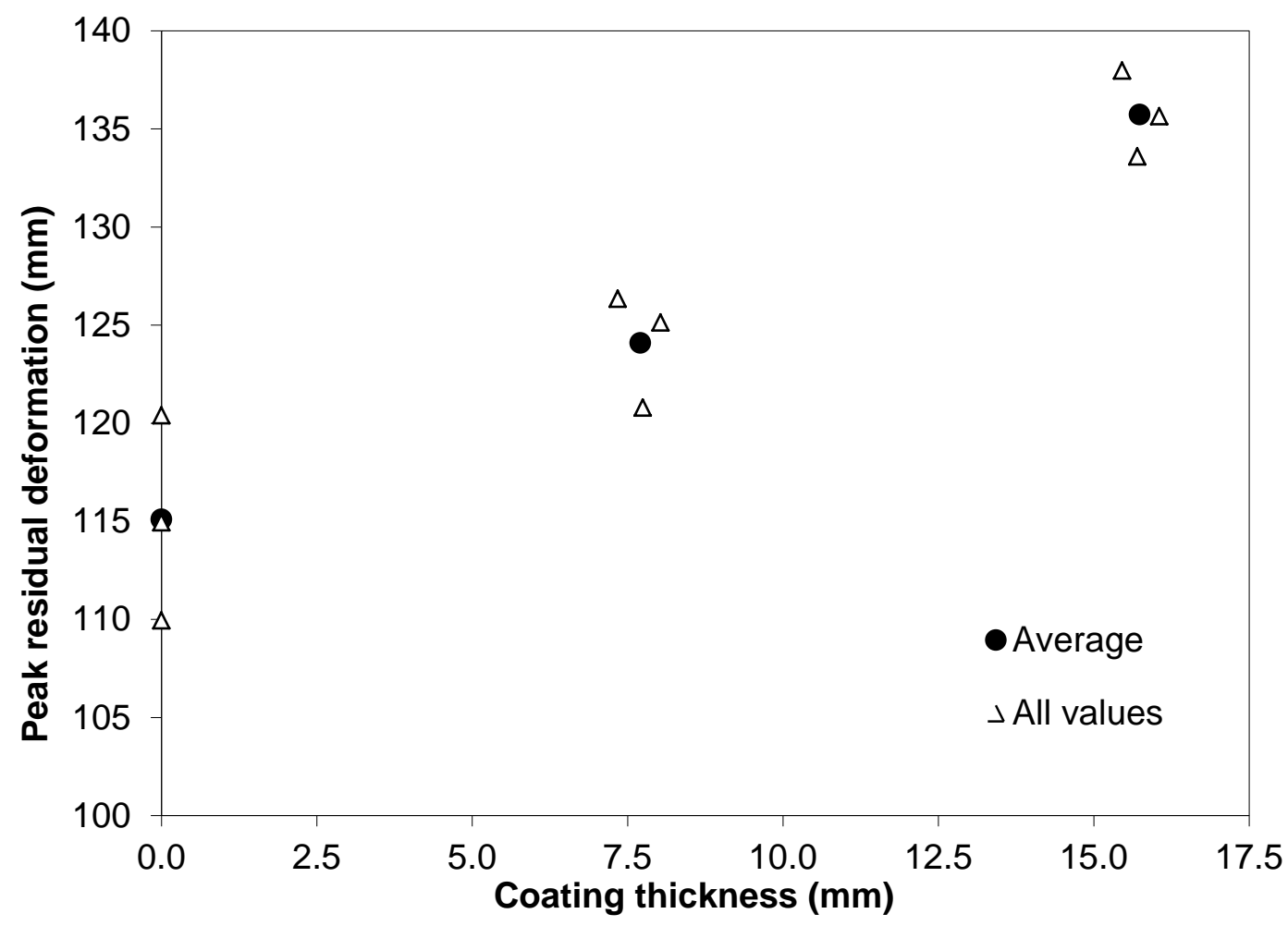

Figure 4.14 Effect of coating thickness on peak residual plate deformation.

\subsubsection{Effect of charge mass on residual deformation}

Figure 4.15 shows the effect of charge mass on plate deformation. If the results for the bare plate only are considered, it can be seen that the deformation increases as the charge mass is increased. This is expected as an increase in charge mass results in an increase in the loading on the plate. However, considering the results for the $4 \mathrm{~mm}$ plates with polymer coatings, the plate deformation was found to decrease slightly with charge mass. When considering only the $5 \mathrm{~mm}$ plates with polymer coatings, no trend is apparent. Therefore although a linear trend could be roughly fitted to the results for the bare plate, the results for the polymer coatings are inconclusive. It is assumed that the charges of higher mass should cause an increase in deformation due to the increased load on the plate; however in this case the results shown below suggest that the effect is negligible for the range of charge masses used and likely to 
be within the scatter of explosive trials. The scatter may be more pronounced for the plates with the polyurea coatings due to the added variables which may arise due to variability in the coating material (e.g. variation in thickness or entrapped bubbles within the coatings) or variations in the bond strength between the coating and the plate. However, it was observed that the radii of the delaminated areas of the $7.7 \mathrm{~mm}$ thick polymer coatings on the $5 \mathrm{~mm}$ plates showed no direct correlation with residual deformation. Likewise, the coating thickness (and therefore density) showed no direct correlation with residual deformation. Thus the effects of all these variables appear to be small enough that they are within the experimental scatter and cannot be assessed individually.

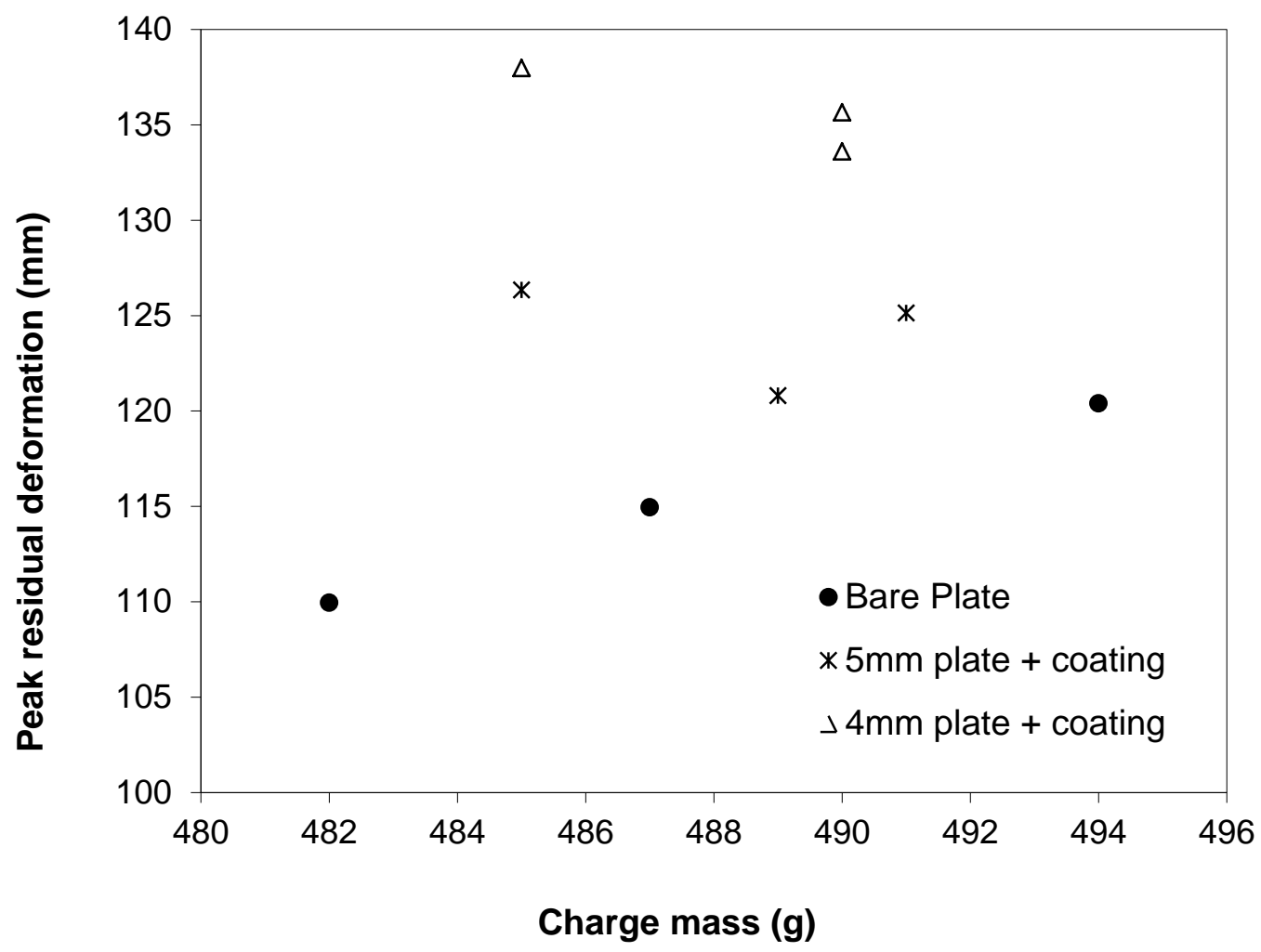

Figure 4.15 Effect of charge mass on peak residual plate deformation.

\subsubsection{Effect of temperature on residual deformation}

Figure 4.16 shows the effect of plate surface temperature on deformation. The $4 \mathrm{~mm}$ plates with polymer coatings show an increase in the deformation with increasing temperature. However, for the bare plates and the $5 \mathrm{~mm}$ plates with polymer coatings, 
the results do not display an obvious trend. These results suggest that for the range of plate temperatures measured during the experiments, any effect is within the experimental scatter.

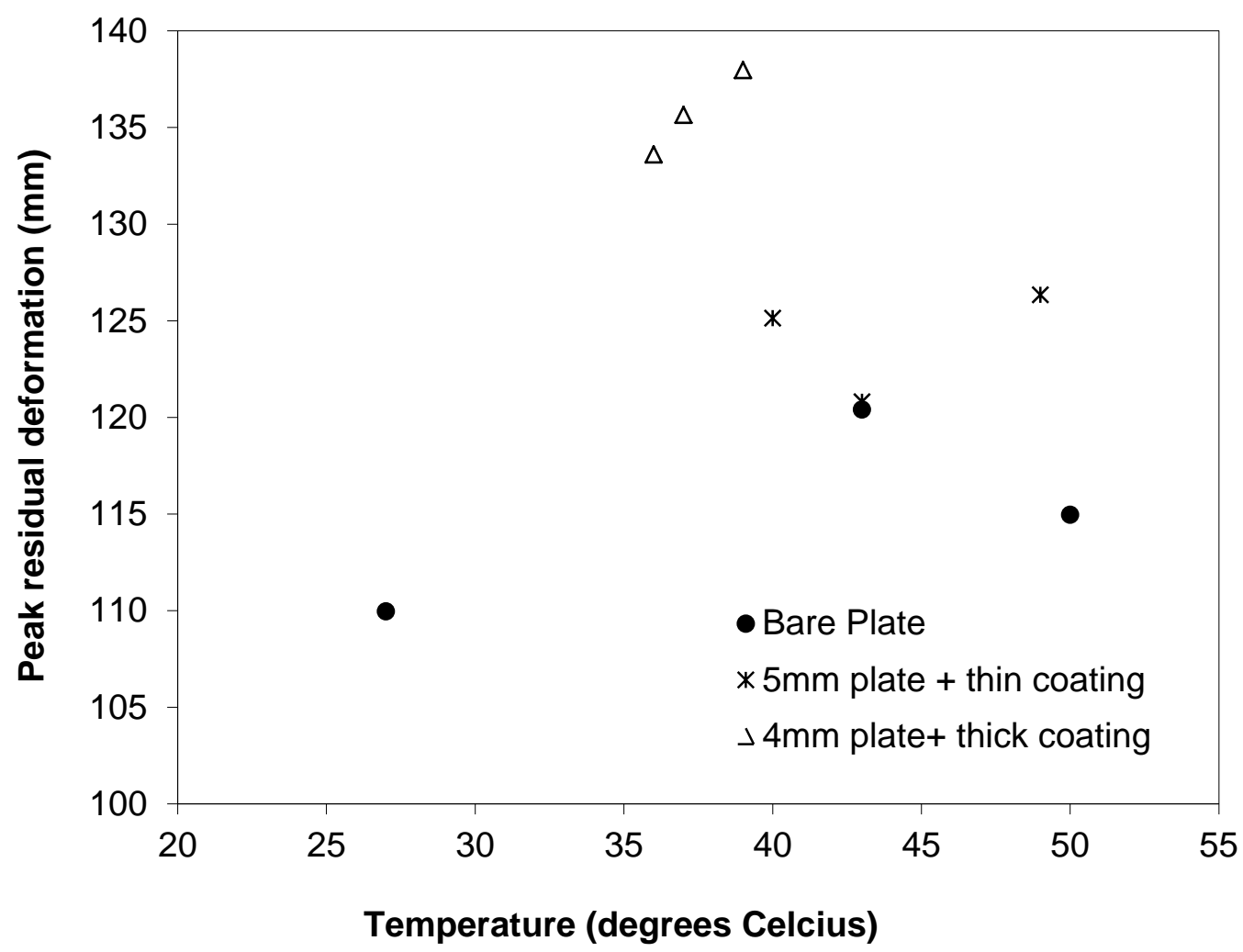

Figure 4.16 Effect of plate temperature on peak residual plate deformation.

\subsubsection{Transient deformations}

The high speed videos recorded by the Miro-3 showed the dynamic movement of the plates during the blast. The Miro-3 camera was used during Events 7 to 10. During Event 7, the plate ruptured due to the use of the mechanical probe, thus no useful images were recorded.

Figure 4.17 shows a sequence of still images taken from the high speed video footage recorded during Event 8 at 0.12 ms intervals. Event 8 was a repeat of Event 7 without the use of the mechanical probe. A maximum transient deformation of $127 \mathrm{~mm}$ was measured from the video footage, occurring at the centre of the plate $1.78 \mathrm{~ms}$ after detonation. It should be noted that this deformation measurement is taken at the back 
surface of the plate (the side opposite to the blast), because the camera was located below the lower surface of the plate. The residual deformation of the plate could not be determined using the high speed video footage because the plate was still moving when the camera stopped recording. Furthermore, the movement of the blast rig caused the camera to move after approximately $4 \mathrm{~ms}$, invalidating any further measurements.

Figure 4.18 shows a sequence of still images from the high speed video footage recorded during Event 9 (plate C3), which tested a $5 \mathrm{~mm}$ plate with a $15.7 \mathrm{~mm}$ polyurea coating. The high speed footage shows de-bonding and hyperextension of the polyurea coating during the event. The maximum extension of the coating was beyond the field of view of the camera. Hence the field of view was adjusted for the following event, resulting in a new framing rate of $6908 \mathrm{fps}$. 


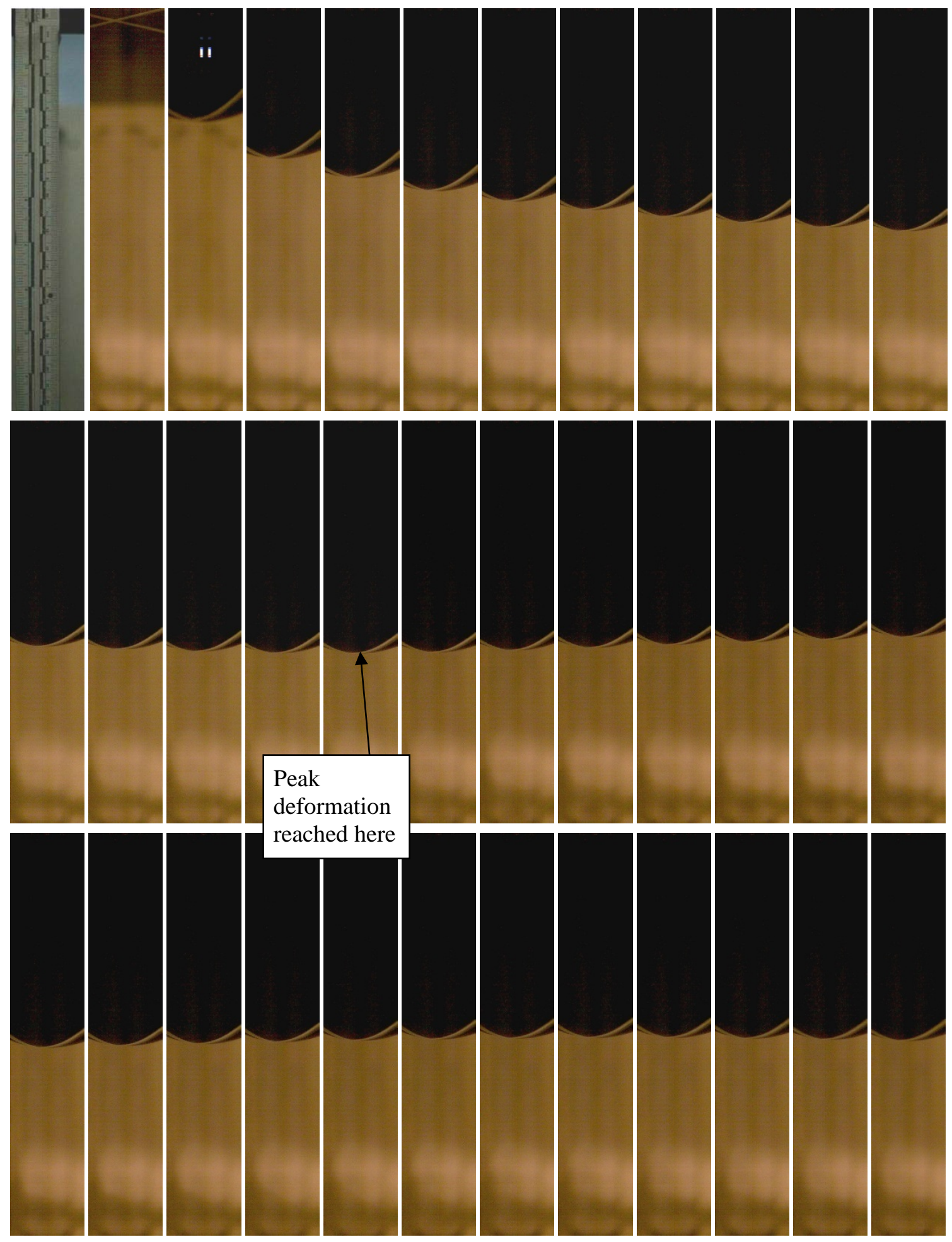

Figure 4.17 Still images recorded by the Phantom Miro-3 high speed camera during Event 8 (plate B4) at $0.12 \mathrm{~ms}$ intervals. The first image shows the image of the scale taken before the plate was attached to the test rig. 
Chapter 4. Explosive Testing of Polyurea-Coated Steel Plates.

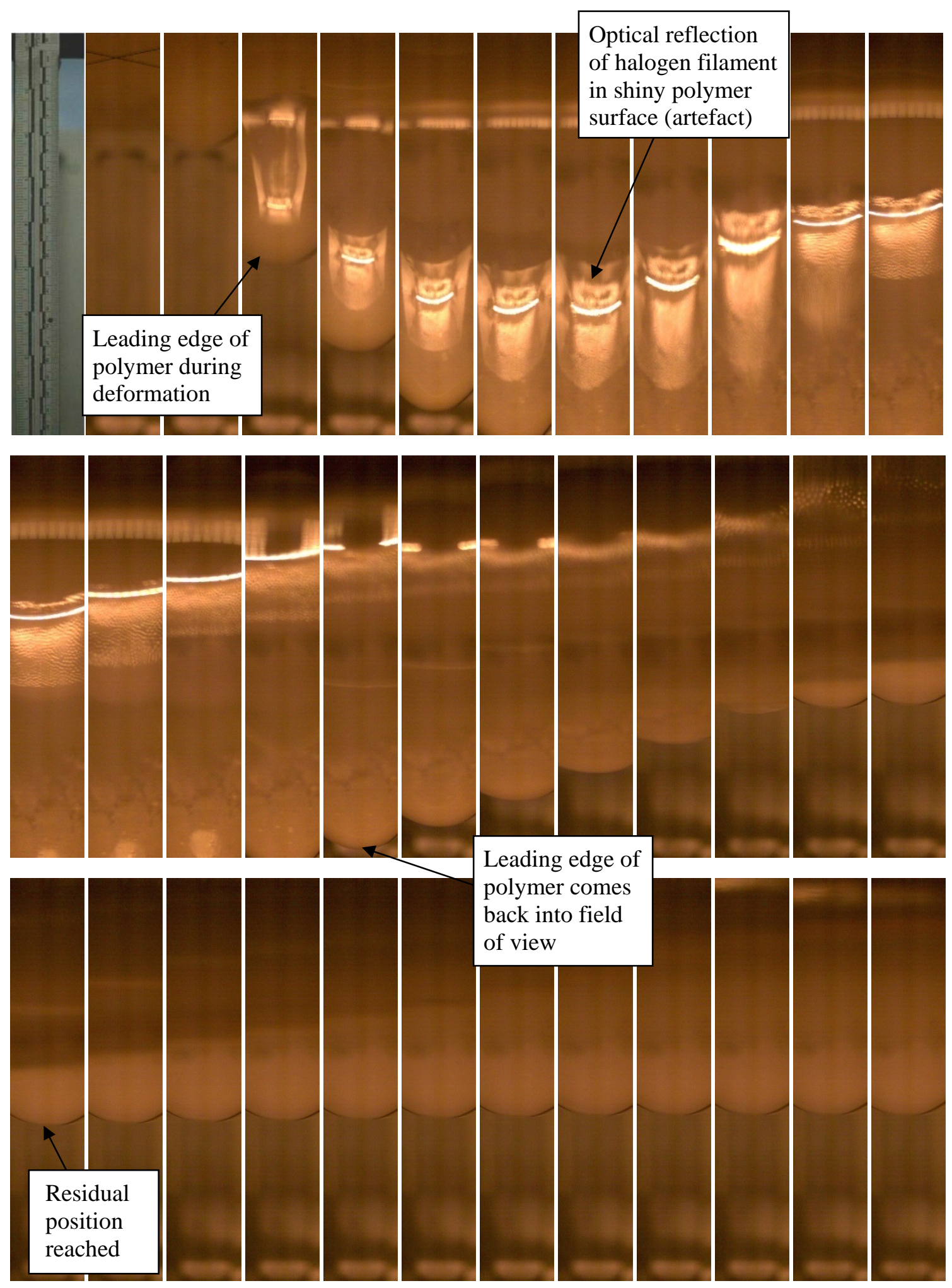

Figure 4.18 Still images recorded by the Phantom Miro-3 high speed camera during Event 9 at $0.12 \mathrm{~ms}$ intervals (plate C3). The first image shows the image of the scale taken before the plate was attached to the test rig. 

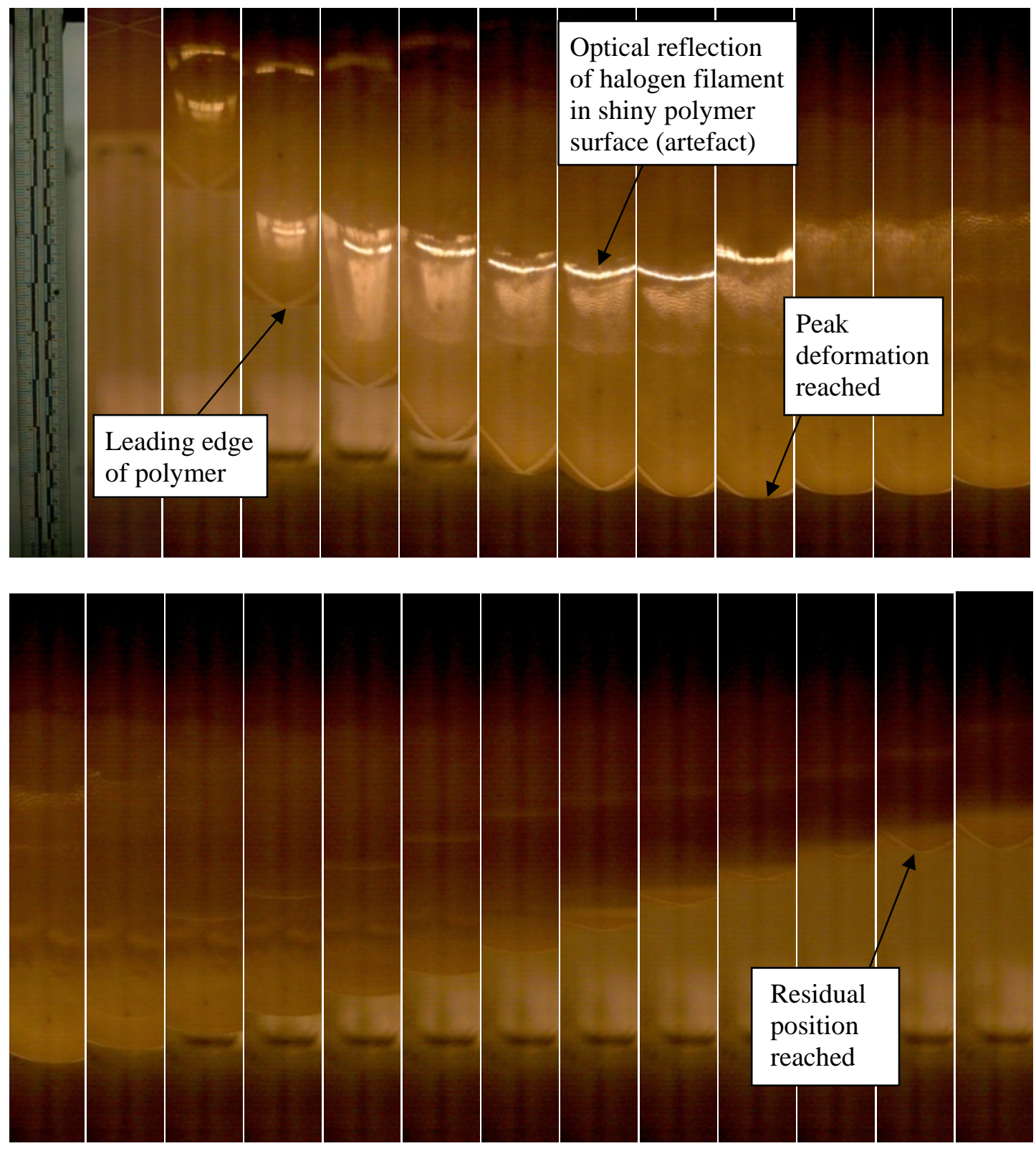

Figure 4.19 Still images recorded by the Phantom Miro-3 high speed camera during Event 10 at $0.14 \mathrm{~ms}$ intervals. The first image shows the image of the scale taken before the plate was attached to the test rig.

Figure 4.19 shows the high speed video footage recorded during Event 10 . The thick polymer coating on a $4 \mathrm{~mm}$ plate used during Event 10 also showed de-bonding and hyperextension. The maximum transient deformation of the polyurea coating was $266 \mathrm{~mm}$ at $1.11 \mathrm{~ms}$.

Figure 4.20 shows a graphical representation of the maximum transient deformation at the plate centre, calculated from the high speed video footage. The point at which the 
polymers are decelerated by resuming contact with the steel plate can be seen as a sudden reduction in the deformation gradient. This occurred at approximately $2.4 \mathrm{~ms}$ after detonation for the thin coating on the $5 \mathrm{~mm}$ plate (C3) and at $3 \mathrm{~ms}$ after detonation for the thick coating on the $4 \mathrm{~mm}$ plate (D3).

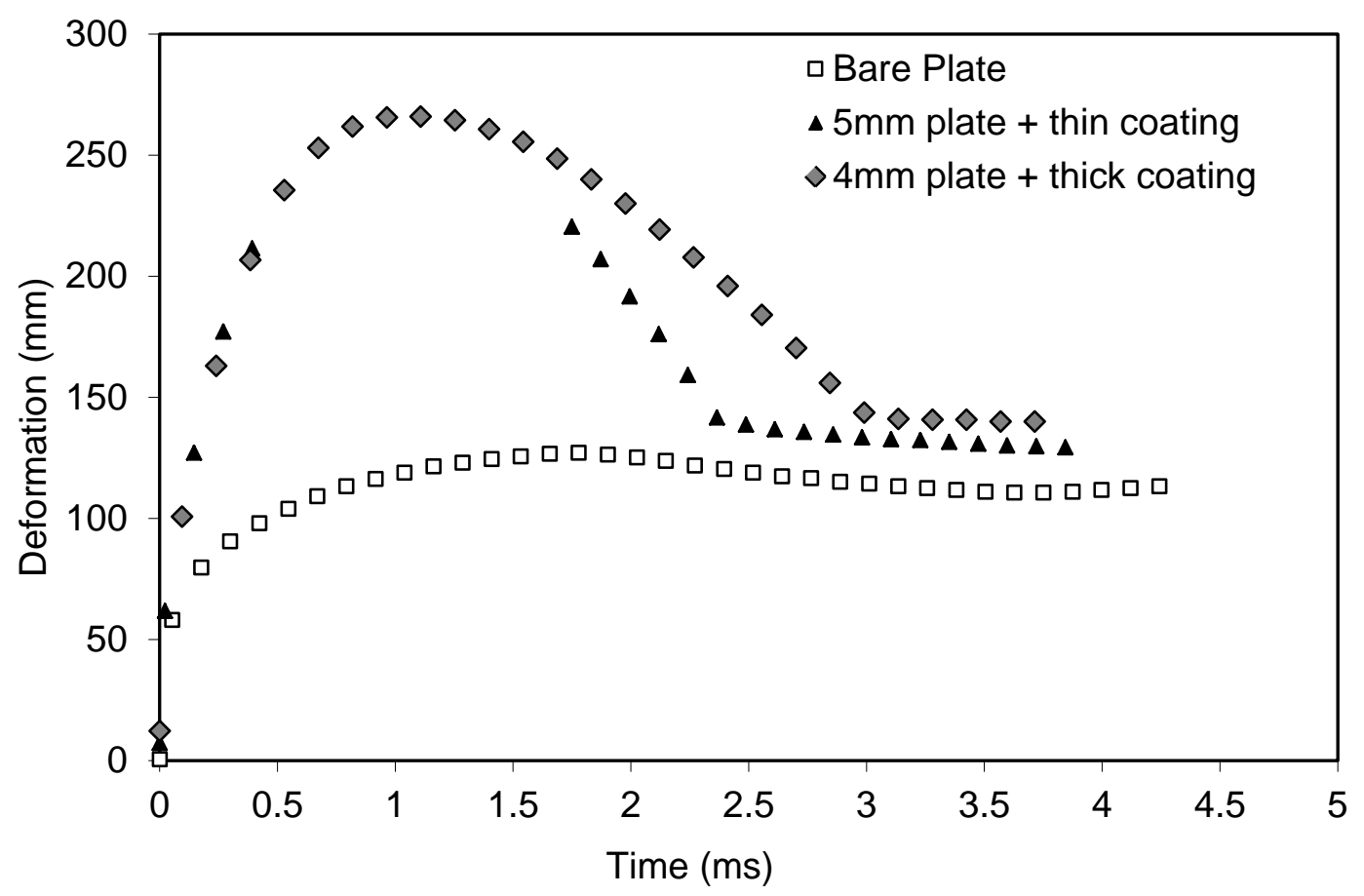

Figure 4.20 Deformation history determined from high speed video footage of Event 8 (plate B4), Event 9 (plate C3) and Event 10 (plate D3).

The maximum transient deformation of the thin polyurea coating on the $5 \mathrm{~mm}$ plate in Event 9 was not recorded, due to the polyurea extending beyond the field of view of the camera. The field of view was adjusted for Event 10, from which maximum transient deformation of the thick polyurea coating was $266 \mathrm{~mm}$ (shown in Figure 4.20). To estimate the peak transient deformation of the polyurea in Event 9, the ratio of the deformation in Event 9 to the deformation in Event 10 was calculated for each capture time. In the $0 \mathrm{~ms}-2.25 \mathrm{~ms}$ domain, the ratio was characterised by a third order polynomial, Figure 4.21. The resulting ratio curve was then applied to estimate the missing Event 9 deformation measurements, shown in Figure 4.22. The resulting peak deformation was $263 \mathrm{~mm}$. A summary of the peak transient deformation results are summarised in Table 4.5. 
Chapter 4. Explosive Testing of Polyurea-Coated Steel Plates.

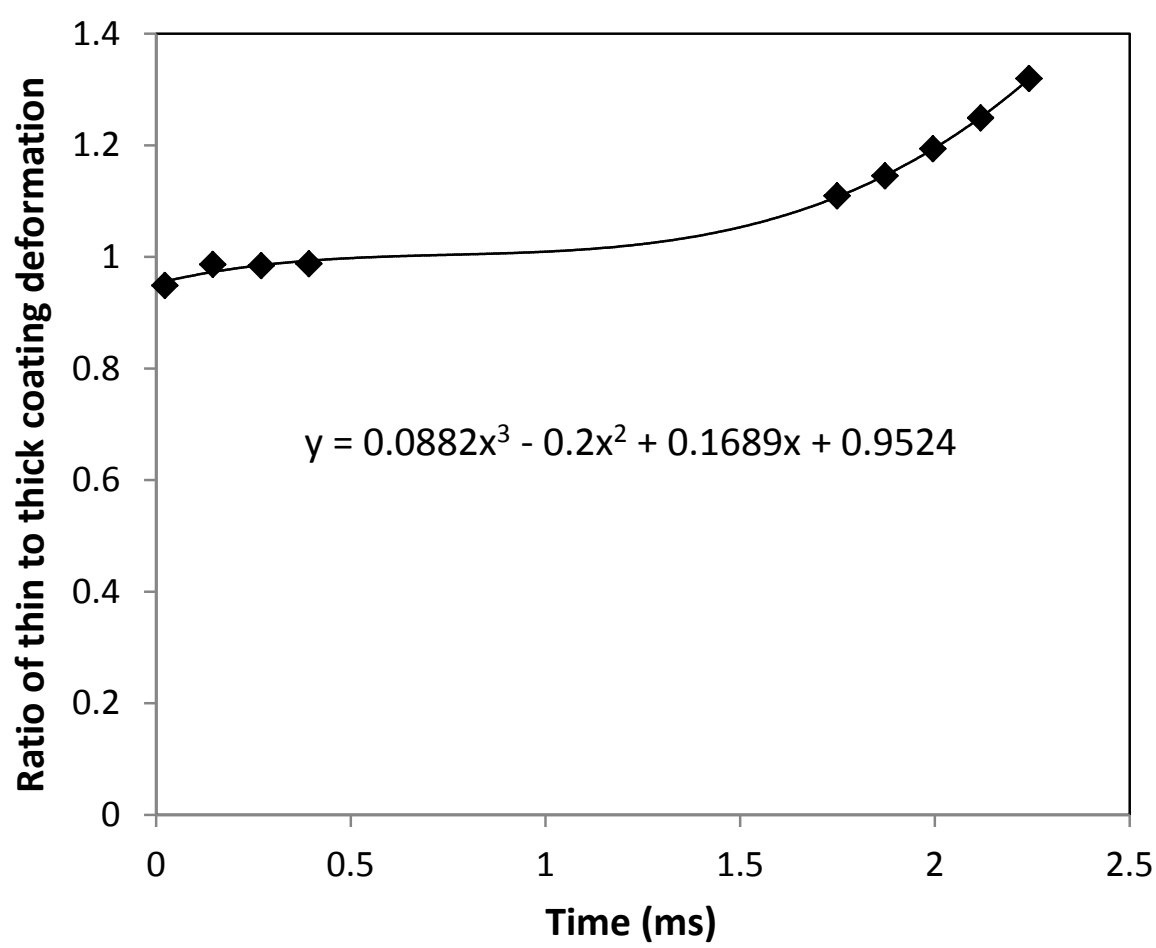

Figure 4.21 Curve fit to the ratio of polyurea deformation in Event 10 to Event 9.

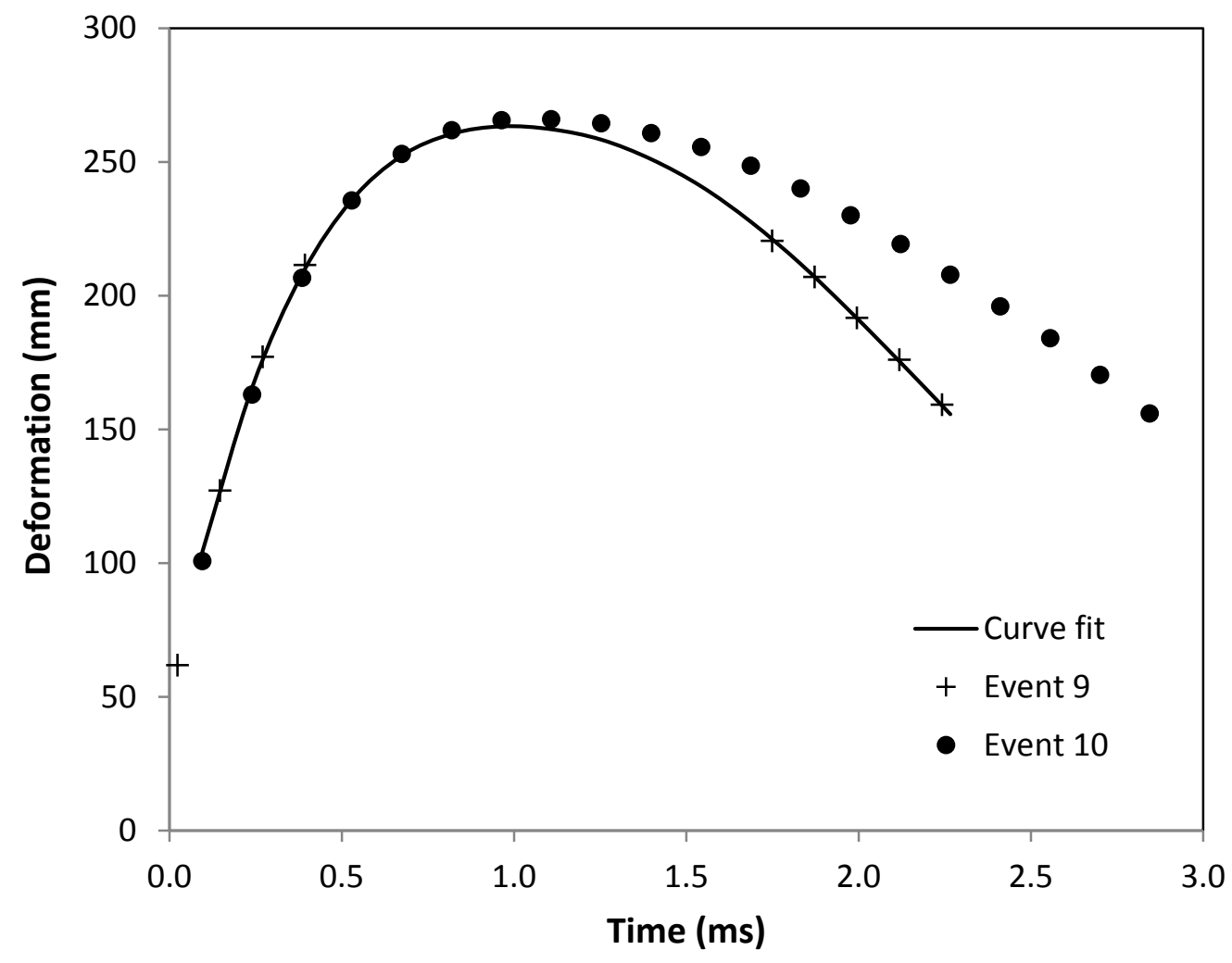

Figure 4.22 Curve fit used to predict the peak deformation of plate C3 (Event 9). 
Chapter 4. Explosive Testing of Polyurea-Coated Steel Plates.

Table 4.5 Peak deformation values calculated from the high speed video.

\begin{tabular}{llll}
\hline Event & Plate & Plate description & $\begin{array}{l}\text { Peak transient } \\
\text { deformation }(\mathrm{mm})\end{array}$ \\
\hline 8 & B4 & Bare 6 mm plate & 127 \\
9 & C3 & 5 mm plate + thin coating & $263^{*}$ \\
10 & D3 & 4 mm plate + thick coating & 266 \\
\hline
\end{tabular}

*Estimated

The laser displacement transducer was used during Events 4 to 7 . A number of triggering problems were encountered during Events 4 to 6 that resulted in no recordings being completed. During Event 7 the plate ruptured resulting in the shattering of the glass covering the laser, rendering it unusable.

The collapsible mechanical probe was positioned within the rig during Event 7 to measure the maximum transient deformation of the plate centre. Instead, the probe appeared to cause the plate to rupture, initiating failure at the point of contact in the plate centre, see Figure 4.13. This was evidenced by copper markings on the plate at the point of initiation and by the high speed camera results. As a result, the mechanical probe was not used in any subsequent events.

\subsubsection{Plate boundary movement measurement}

The boundaries of the plates were spray painted in order to determine the degree of slippage and stretching at the plate boundaries during the blast event. As shown in Figure 4.23. 


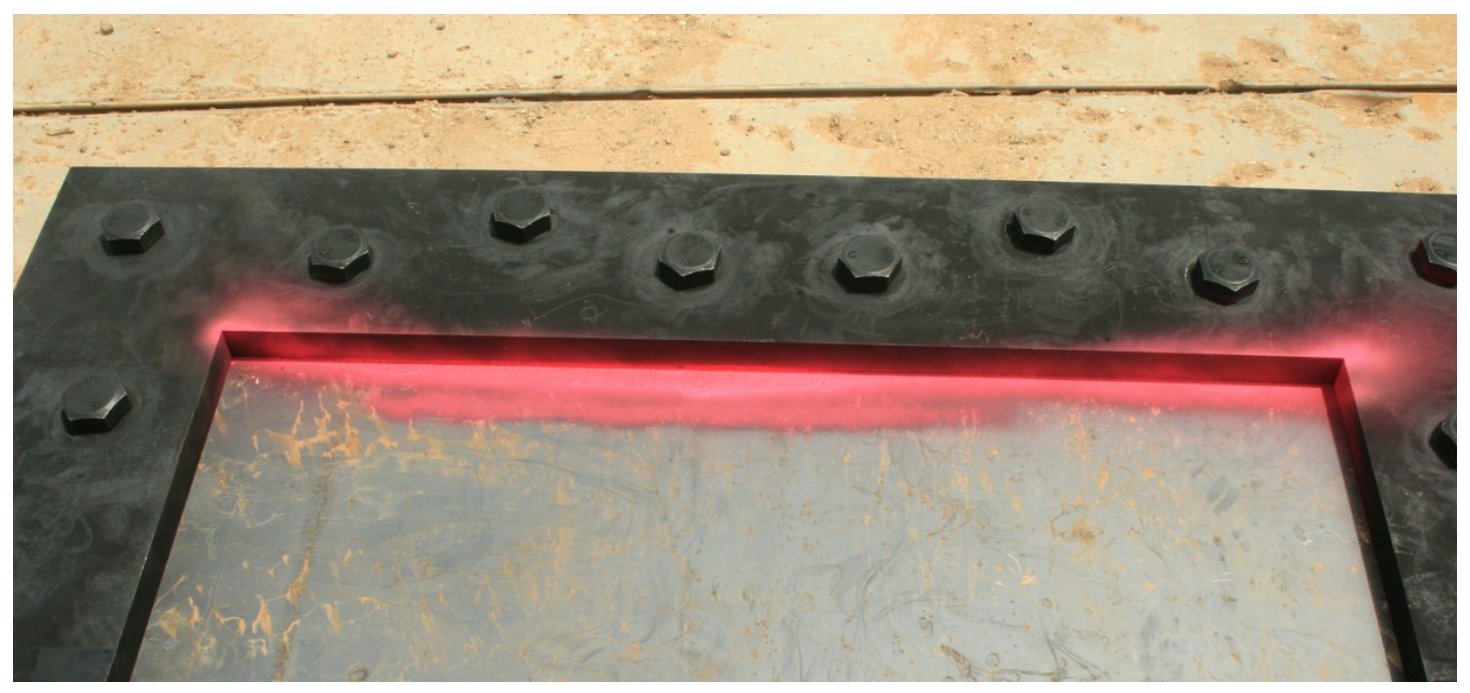

Figure 4.23 Pink spray paint was applied to the plate boundaries to indicate the amount of slippage that occurred during each event (pre-event image).

The boundaries of the plate were found to move a maximum of 2 to $3 \mathrm{~mm}$ during each event, typically near the middle of each edge span, as shown in Figure 4.24. The image on the left shows the boundary before the event. The image on the right shows the boundary after the event, with the gap between the pink areas showing where the top surface of the plate has moved from its original position. This movement is likely due to a combination of slippage at the bolt holes ( $27 \mathrm{~mm}$ diameter holes and $25 \mathrm{~mm}$ diameter bolts were used), and the stretching of the plate between the bolt holes and the clamping boundary. This slippage will cause a slight increase in plate deformation, compared to an ideal clamped boundary (as used in the numerical models). 

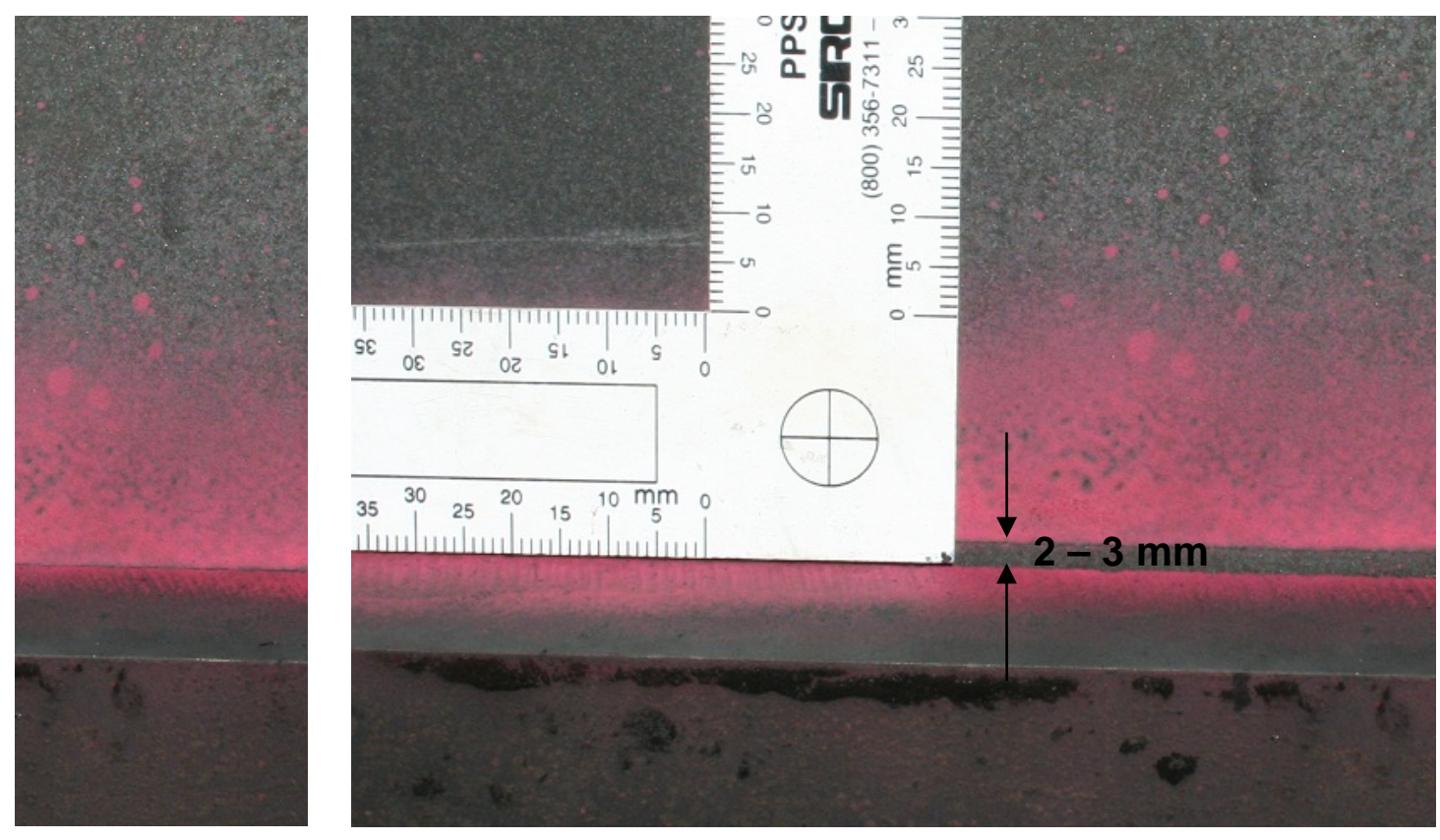

Figure 4.24 The left hand image shows the plate boundary before an event. The right hand image shows the plate boundary after the event, with plate boundary movement as indicated.

\subsection{Summary}

The aim of the experimental trial was to determine whether the application of polyurea coatings to steel plates can reduce their deformation under blast loading, compared to increasing the steel plate thickness to an equivalent areal density. It was found that for the configurations used during this trial, the application of a polymer coating did not reduce the residual plate deformation when compared to increasing the steel plate thickness. Rather, it resulted in an increase of the residual deformation caused by the blast loading. As the polyurea to steel ratio was increased, the residual deformation also increased. There was some variation in residual deformations between the repeated tests, which may be due to a combination of charge mass effects, temperature effects, coating variability and possible variability in the yield strength of the steel plates. However, the observed trend of increasing deformation is well outside the recorded experimental scatter, and is hence a clear result.

In the preliminary trials without high speed video footage (Chapter 3), the performance of the coatings was measured solely by the plate residual deformations. 
Whilst residual measurements give some indication of performance, the transient deformation is a better indicator of blast resistance as this defines the damage zone behind the plate. The high speed video footage showed evidence of the polyurea coatings hyper-extending well beyond the peak deformation of the steel plate. The thicker polyurea coating was found to have a slightly higher transient deformation compared to the thinner coating.

The results presented here have provided experimental data which will be used to validate numerical models in the next chapter. 


\section{Chapter 5}

\section{Numerical Simulations and Parametric Studies}

\subsection{Introduction}

Numerical simulations were conducted to develop a deeper understanding of the response of polyurea-coated plates to blast loading. The hydrocode ANSYS ${ }^{\circledR}$ AUTODYN ${ }^{\circledR}$ was used to simulate the interaction of the blast load and plate response using a coupled fluid-structure analysis. The use of hydrocodes for such simulations, as well as the material models used in this chapter, was introduced in the literature review (Chapter 2). The first aim in conducting the numerical simulations was to validate the application of such numerical models for very close-in (detonics regime) blast loading by comparison with the experimental results presented in Chapter 4. Although preliminary models were developed earlier in Chapter 3, they did not simulate the polyurea de-bonding and large transient deformations which were observed using high speed photography in the blast experiments. Further aims were to develop improved material model constants for polyurea, and to gain a better understanding of how different properties affect the response of polyurea-coated plates to blast loading. This was achieved by using the validated numerical models to conduct parametric studies to determine how coating stiffness, location, bond strength and bulk modulus affect the blast performance of polyurea-coated plates.

\subsection{Numerical modelling of the explosive experiments}

This section describes the initial numerical simulation of the explosive experiments presented in Chapter 4. 


\subsubsection{Mesh discretisation}

To simulate the blast load on the XLERPLATE 350 steel plates (tests B1-B3), the air was modelled using a 3D Eulerian grid in quarter symmetry. The dimensions of the grid were $350 \mathrm{~mm} \times 350 \mathrm{~mm} \times 200 \mathrm{~mm}$, discretised into 1,452,000 elements. Figure 5.1 shows the mesh, which was weighted so there were smaller elements $(1.3 \times$ $1.3 \times 1.3 \mathrm{~mm}$ ) where the highest pressures were expected. The largest elements located at the volume boundaries were $13 \mathrm{~mm} \times 13 \mathrm{~mm} \times 8 \mathrm{~mm}$. The $\mathrm{X}=0$ and $\mathrm{Y}=0$ planes were set as symmetry planes, and a "flow out" condition was applied to the remaining four surfaces of the grid.

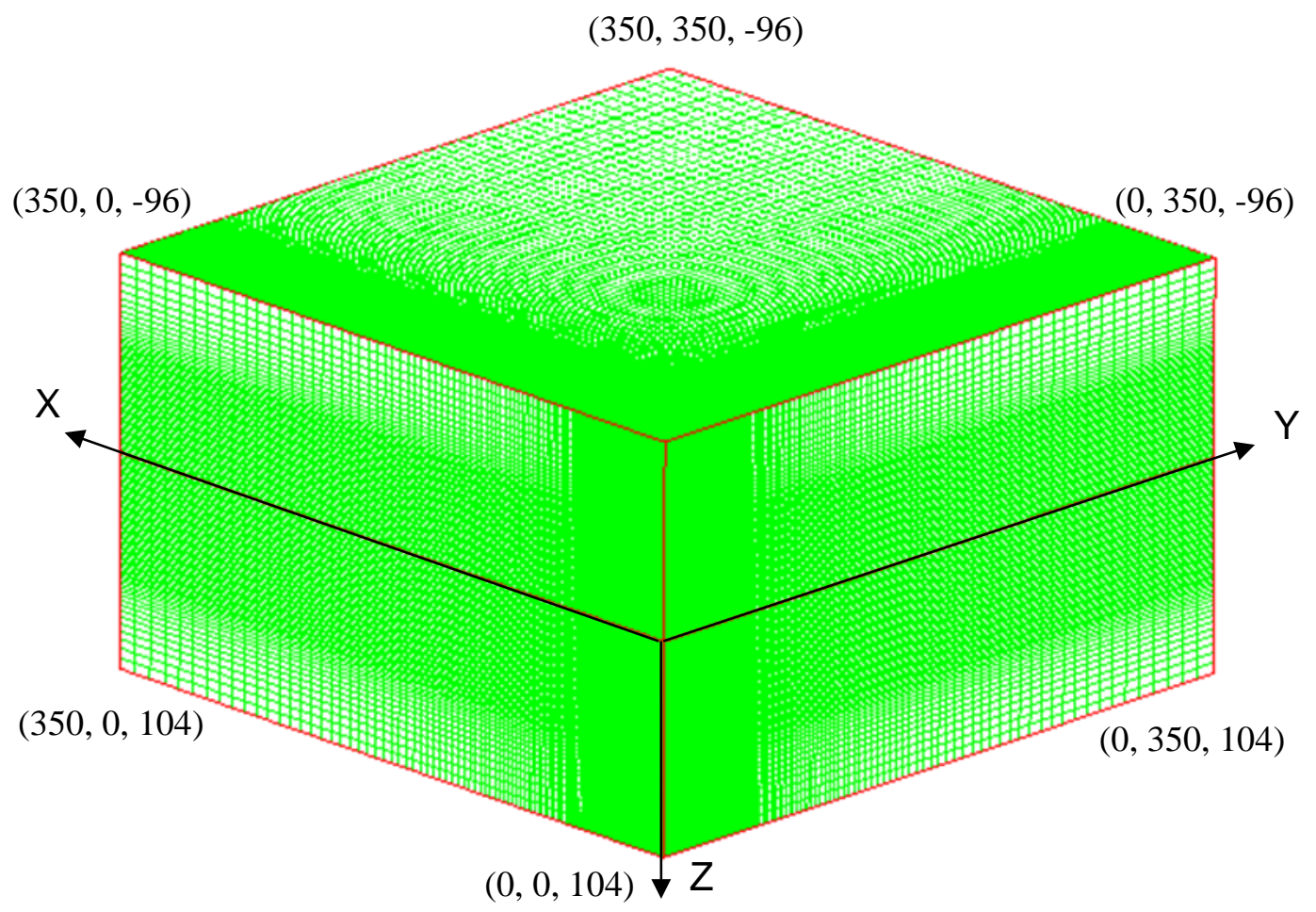

Figure 5.1 Eulerian mesh used to model air-blast on plates. The solid green areas contain elements too small to be distinguished due to the resolution of the image. Dimensions are in $\mathrm{mm}$.

As for the validation models, the explosive was first modelled as a 1D wedge until the radius of the shock wave was such that it was about to interact with the plate. At this point the $1 \mathrm{D}$ wedge was remapped onto the $3 \mathrm{D}$ grid at a stand-off representing a $10 \mathrm{~mm}$ gap between the original surface of the explosive and the plate. 
The lateral dimensions of the plate in the quarter symmetry model were $350 \mathrm{~mm} \times 350 \mathrm{~mm}$. The plate was discretised into $5 \mathrm{~mm} \times 5 \mathrm{~mm}$ shell elements, with an artificial thickness corresponding to the test plate (i.e. $4 \mathrm{~mm}, 5 \mathrm{~mm}$ or $6 \mathrm{~mm}$ ). The non-symmetry boundaries of the plates were fixed to represent the clamped edges by setting all translational and rotational velocities to zero at the nodes. The EulerLagrange interaction was set to fully coupled, to allow the air-blast and plate to interact.

The polyurea was modelled using 8-node hex elements to capture the through thickness stress distribution. The width and length of the elements were set to $5 \mathrm{~mm} \times 5 \mathrm{~mm}$ so that the nodes of the polyurea were aligned with the nodes of the steel plate, thus allowing the polyurea and plate to be joined. The polyurea thicknesses were $7.7 \mathrm{~mm}$ and $15.7 \mathrm{~mm}$ for the thin and thick coatings, respectively. The polyurea reference density was set to $1.003 \mathrm{~g} / \mathrm{cm}^{3}$, based on the average density of the polyurea used in the experiment. Four elements were used through the thickness of the thin coating and eight were used for the thick coating. The polyurea was modelled over an area of $335 \times 335 \mathrm{~mm}$ to match the experiment.

The simulations were run until the time reached $0.3 \mathrm{~ms}$, at which point the air was removed from the model. After this time the blast loading of the plate had completed, and the removal of the air sped up the calculation time significantly. A static damping coefficient of $5.5 \times 10^{-4}$ was applied at $10 \mathrm{~ms}$ to allow the plate deformation to converge to a static solution.

\subsubsection{Material modelling}

The material models for air and pentolite were the same as those used in Chapter 3. They were taken from the AUTODYN material library and used ideal gas and JWL equations of state, respectively. The plate material was modelled using a JohnsonCook strength model, with constants as defined in Chapter 3, Section 3.3.2. Initial models used a yield strength of $360 \mathrm{MPa}$, based on the minimum tensile strength of the Bluescope XLERPLATE 350 steel. To verify this material parameter, quasi-static tensile tests were performed on the three thicknesses of plate to be tested ( $4 \mathrm{~mm}$, $5 \mathrm{~mm}$, and $6 \mathrm{~mm}$ ). Plates of $4 \mathrm{~mm}$ thickness were tested from both the preliminary 
experiments and the Chapter 4 explosive blast tests, as the steel for these experiments was sourced from different batches. Tensile test specimens were machined from cutoffs from the test plates and tested according to ASTM standard E 8/E 8M-08, Standard Test Methods for Tension Testing of Metallic Materials (ASTM International, 2008). Figure 5.2 shows the dimensions of the test specimens. Five specimens were produced in the rolling direction and five in the transverse direction for each of the plates. The tests were performed using an Instron ${ }^{\circledR} 1185$ tensile testing machine with a maximum load of $100 \mathrm{kN}$. A summary of the tensile test results is shown in Table 5.1. The full tensile test results can be found in Appendix D. 


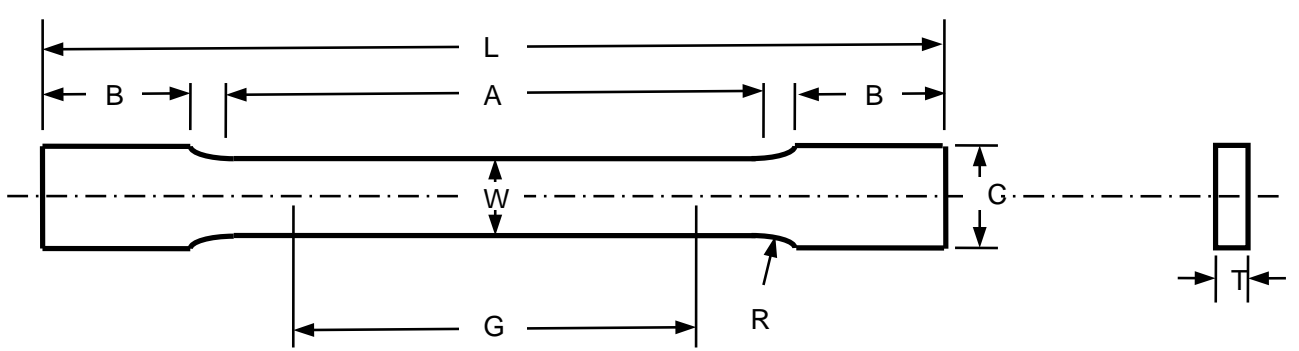

Dimensions $\mathrm{mm}$

G-Gage length $\quad 50.0 \pm 0.1$

W-Width $\quad 12.5 \pm 0.2$

T-Thickness thickness of material $(4,5$ or $6 \mathrm{~mm})$

$\mathrm{R}$-Radius of fillet $\quad 12.5$

L-Overall length 200

A-Length of reduced section $\quad 57$

B-Length of grip section $\quad 50$

C-Width of grip section, $\quad 20$

approximate

Figure 5.2 Dimensions used for tensile test specimens as per ASTM standard E 8/E 8M-08. 
Chapter 5. Numerical Simulations and Parametric Studies.

Table 5.1 Results of tensile tests on the XLERPLATE 350 steel.

\begin{tabular}{|c|c|c|c|c|c|}
\hline $\begin{array}{l}\text { Nominal } \\
\text { specimen } \\
\text { thickness } \\
(\mathrm{mm})\end{array}$ & $\begin{array}{l}\text { Average } \\
\text { measured } \\
\text { specimen } \\
\text { thickness (mm) }\end{array}$ & $\begin{array}{l}\text { Min } 0.2 \% \\
\text { offset Yield } \\
\text { Stress } \\
(\mathrm{MPa})\end{array}$ & $\begin{array}{l}\text { Max } 0.2 \% \\
\text { offset Yield } \\
\text { Stress } \\
(\mathrm{MPa})\end{array}$ & $\begin{array}{l}\text { Average } \\
0.2 \% \text { offset } \\
\text { Yield Stress } \\
(\mathrm{MPa})\end{array}$ & $\begin{array}{l}\text { Standard } \\
\text { deviation } \\
(\mathrm{MPa})\end{array}$ \\
\hline $4^{*}$ & 3.97 & 348 & 384 & 366 & 11 \\
\hline $4^{* *}$ & 3.86 & 380 & 412 & 402 & 12 \\
\hline 5 & 4.89 & 377 & 416 & 400 & 15 \\
\hline 6 & 5.91 & 434 & 468 & 446 & 12 \\
\hline
\end{tabular}

*Test specimens from validation experiments.

${ }^{* *}$ Test specimens from Chapter 4 explosive blast tests.

A range of yield strengths were measured during the tensile tests, demonstrated by the maximum and minimum values and standard deviations in Table 5.1. Furthermore, the two $4 \mathrm{~mm}$ thick Bluescope XLERPLATE 350 plates produced significantly different results, indicating variations in properties between the steel batches. No significant difference in tensile properties was found between the rolling and transverse directions.

The polyurea was modelled using the 2-parameter Mooney-Rivlin hyperelastic strength model, with constants introduced in Chapter 3, Section 3.3.2.

\subsubsection{Modelling of polyurea de-bonding}

During the experiments the polyurea coatings were observed to de-bond from the plates during the blast events. To simulate this, a simple breakable bond was modelled between the polyurea and the plate. 
To simulate the observed de-bonding, two new meshes for the plate and polyurea were generated, as shown in Figure 5.3, corresponding to the two coating thicknesses tested. The grid was designed to match the circular de-bonding profile observed in the experiments. The plate meshes were weighted such that there were smaller elements near the plate centres. The size of the elements at the plate centres was $1.8 \mathrm{~mm} \times 1.8 \mathrm{~mm}$, increasing to $5 \mathrm{~mm} \times 5 \mathrm{~mm}$ at the boundaries. The mesh for the thin coating had four elements through its thickness, whereas the mesh for the thick coating contained eight. This gave an aspect ratio of close to one for the elements near the plate centre. A very thin layer $(0.2 \mathrm{~mm})$, single layer of solid elements was created between the plate and the polyurea to represent a breakable bond between the two materials, Figure 5.4. The bond material was given the same material properties as the polyurea, but with a principal stress failure model added. This means that when the bond material reaches the specified failure stress, the material will fail and its ability to carry stress reduces to zero. 
Chapter 5. Numerical Simulations and Parametric Studies.

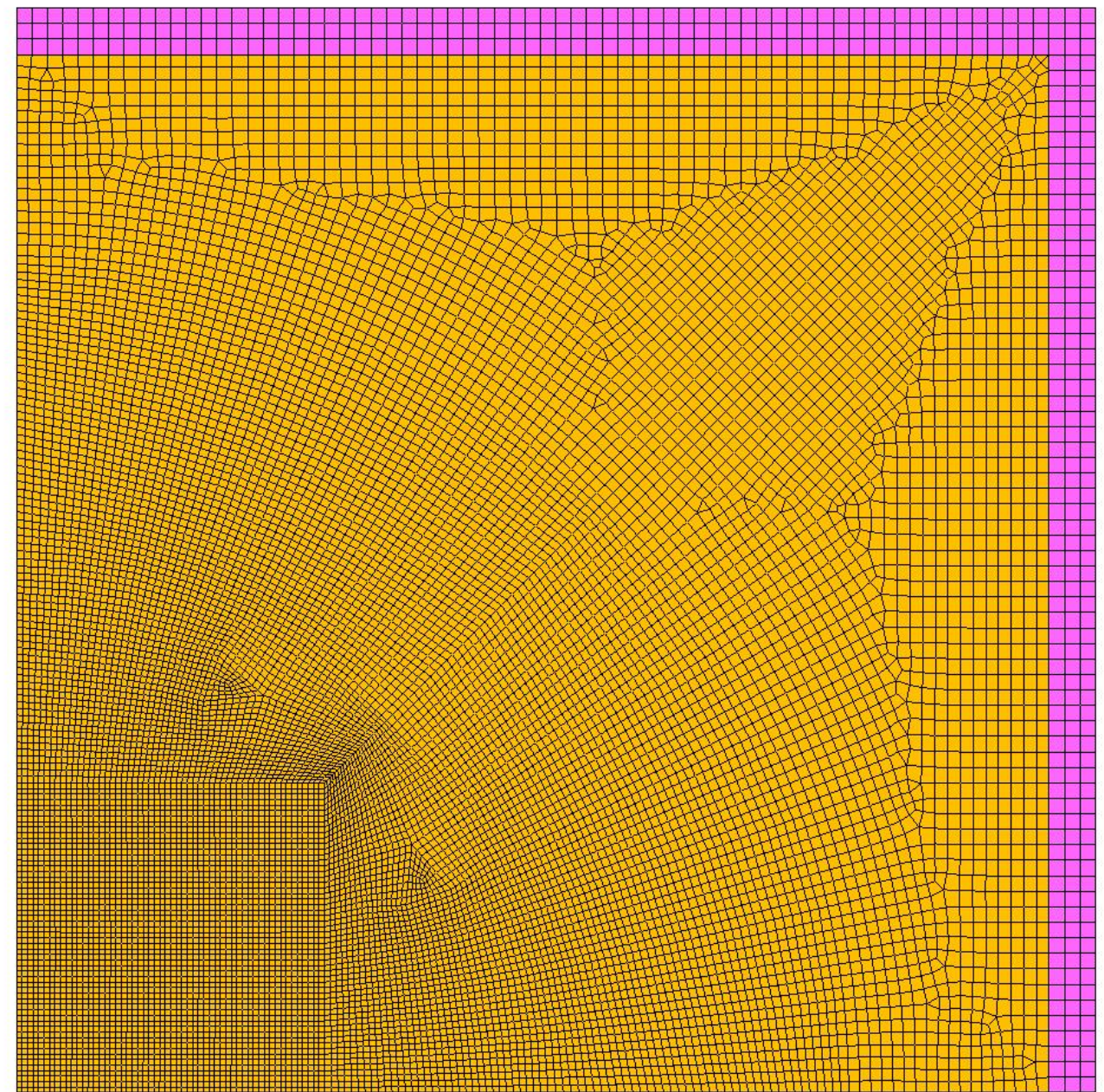

Figure 5.3 Mesh used for modelling polyurea de-bonding from the plate during the blast. The orange area represents the polyurea and the pink area shows the outer edge of the steel plate.
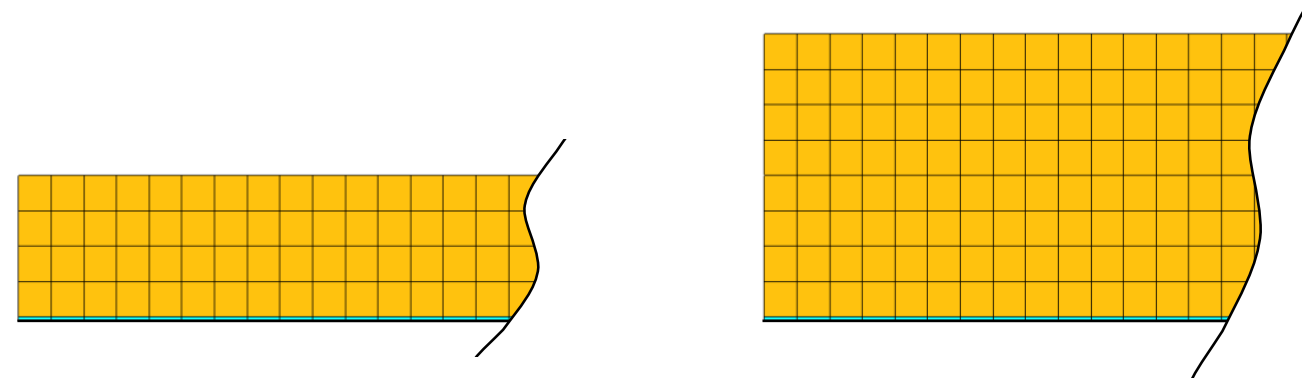

Figure 5.4 Through thickness mesh showing the polyurea (in orange) and the bond material (in light blue). The steel plate is not shown here. 
As the actual bond strength between the steel and the polyurea was unknown, an inital failure stress similar to that of steel was estimated, equalling $400 \mathrm{MPa}$. Although this was expected to be a gross over-prediction, it was chosen to ensure that the debonding would not extend to the edge of the polyurea and thus cause the whole polyurea coating to break away from the plate.

In Figure 5.5, the model (with an assumption of a $400 \mathrm{MPa}$ bond strength) is demonstrating successful de-bonding of the thin polyurea coating. After reaching its maximum extension, the polyurea returned to the back of the plate. The radius of the de-bonded area was $38 \mathrm{~mm}$, compared to the average experimental result of $247.5 \mathrm{~mm}$, suggesting an overestimate of the bond strength.

Figure 5.5 Polyurea-coated plate model showing the initiation of polyurea debonding. The light blue region represents the void caused by local debonding.

A series of three simulations were performed in which the bond strength was decreased from its initial value of $400 \mathrm{MPa}$. This resulted in a collection of data points for the de-bonding radius based on yield stress, plotted in Figure 5.6. An exponential curve was fitted to the data in order to estimate the failure stress that would cause a de-bonding radius of $247.5 \mathrm{~mm}$. The curve was extrapolated to give an estimated failure stress of $90 \mathrm{MPa}$. This is similar to the value found by Amini et al. (2010a), who modelled polyurea-coated steel plates and found that a shear failure strength of the bond between the polyurea and the plate of $100 \mathrm{MPa}$ gave the best agreement with their experimental results. 
Chapter 5. Numerical Simulations and Parametric Studies.

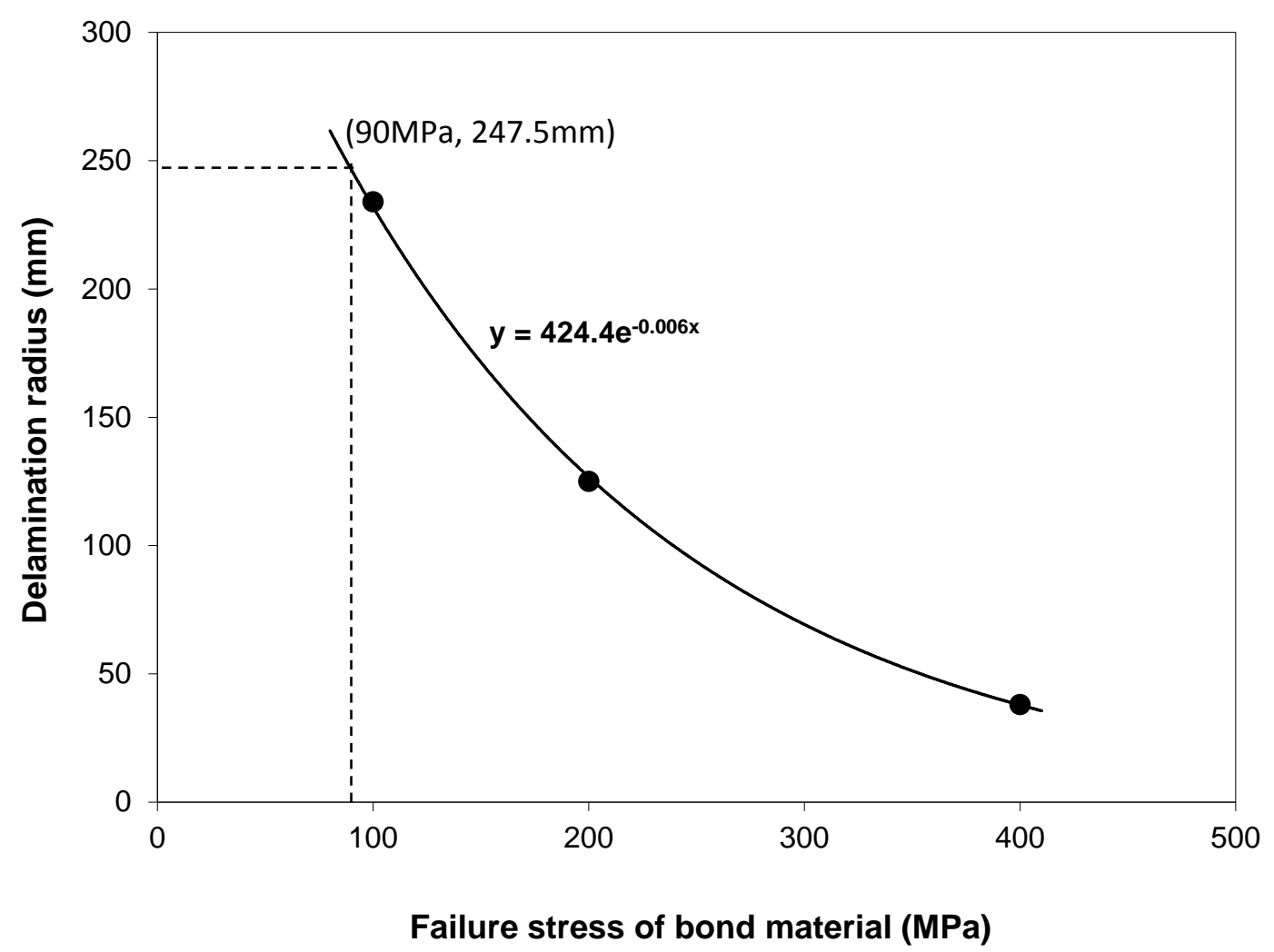

Figure 5.6 Results of numerical modelling showing the effect of the bond failure stress on the radius over which the polyurea de-bonds.

To validate the extrapolation, a simulation was run with a bond failure stress of $90 \mathrm{MPa}$, resulting in a de-bonding radius of $245 \mathrm{~mm}$ for the thin polyurea coating on the $5 \mathrm{~mm}$ plate, which is close to the experimental average of $247.5 \mathrm{~mm}$. Figure 5.7 and Figure 5.8 show a series of screen captures from the simulation showing the polyurea de-bonding. The $7.7 \mathrm{~mm}$ polyurea coating extended to a peak deformation of $197 \mathrm{~mm}$ before coming to rest back against the steel plate. 

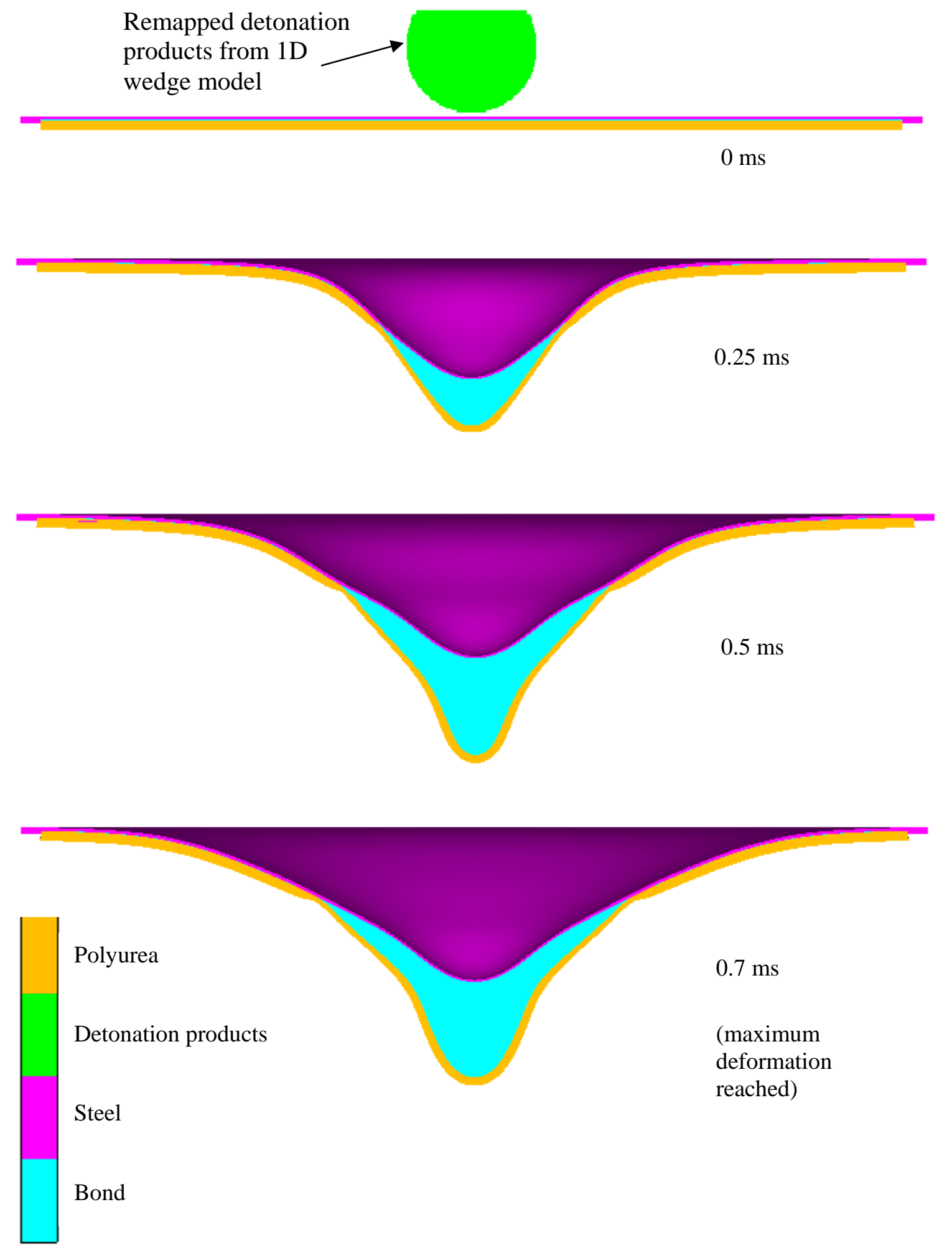

Figure 5.7 Polyurea de-bonding for the $5 \mathrm{~mm}$ steel plate with a $7.7 \mathrm{~mm}$ thick polyurea coating. The light blue region represents the void caused by local de-bonding. 
Chapter 5. Numerical Simulations and Parametric Studies.
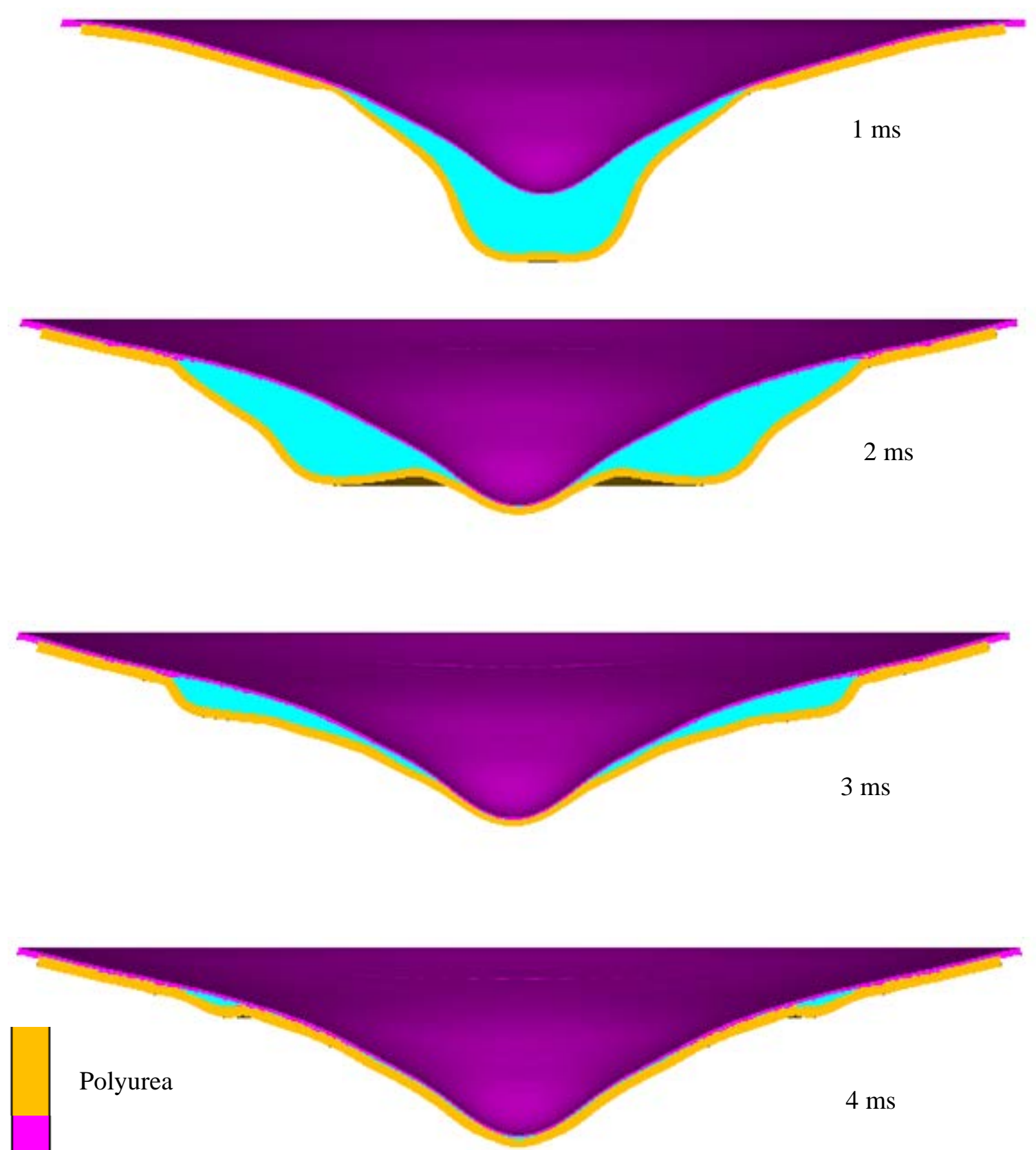

Steel

Bond

Figure 5.8 Polyurea de-bonding (continued) for the $5 \mathrm{~mm}$ steel plate with a $7.7 \mathrm{~mm}$ thick polyurea coating. The polyurea is shown to "spring back", coming to rest against the plate. 
Having determined a suitable bond failure stress, a simulation was then run using the mesh for the thick $(15.7 \mathrm{~mm})$ coating, with a failure stress of $90 \mathrm{MPa}$ applied to the bond material. Figure 5.9 and Figure 5.10 show a series of screen captures showing the de-bonding of the $15.7 \mathrm{~mm}$ thick polyurea. The polyurea de-bonded over a large area, with only the corners remaining attached to the plate, which was consistent with the experimental results. This simulation was not run to completion as the time step became too small after $\sim 8 \mathrm{~ms}$. However, it was observed that by this time the plate centre was no longer moving (noting also that static damping had been applied at $5 \mathrm{~ms}$ ), hence the residual deformation at the plate centre was assumed to have been reached. The $15.7 \mathrm{~mm}$ polyurea coating extended to a peak deformation of $227 \mathrm{~mm}$ before coming to rest back against the steel plate. 

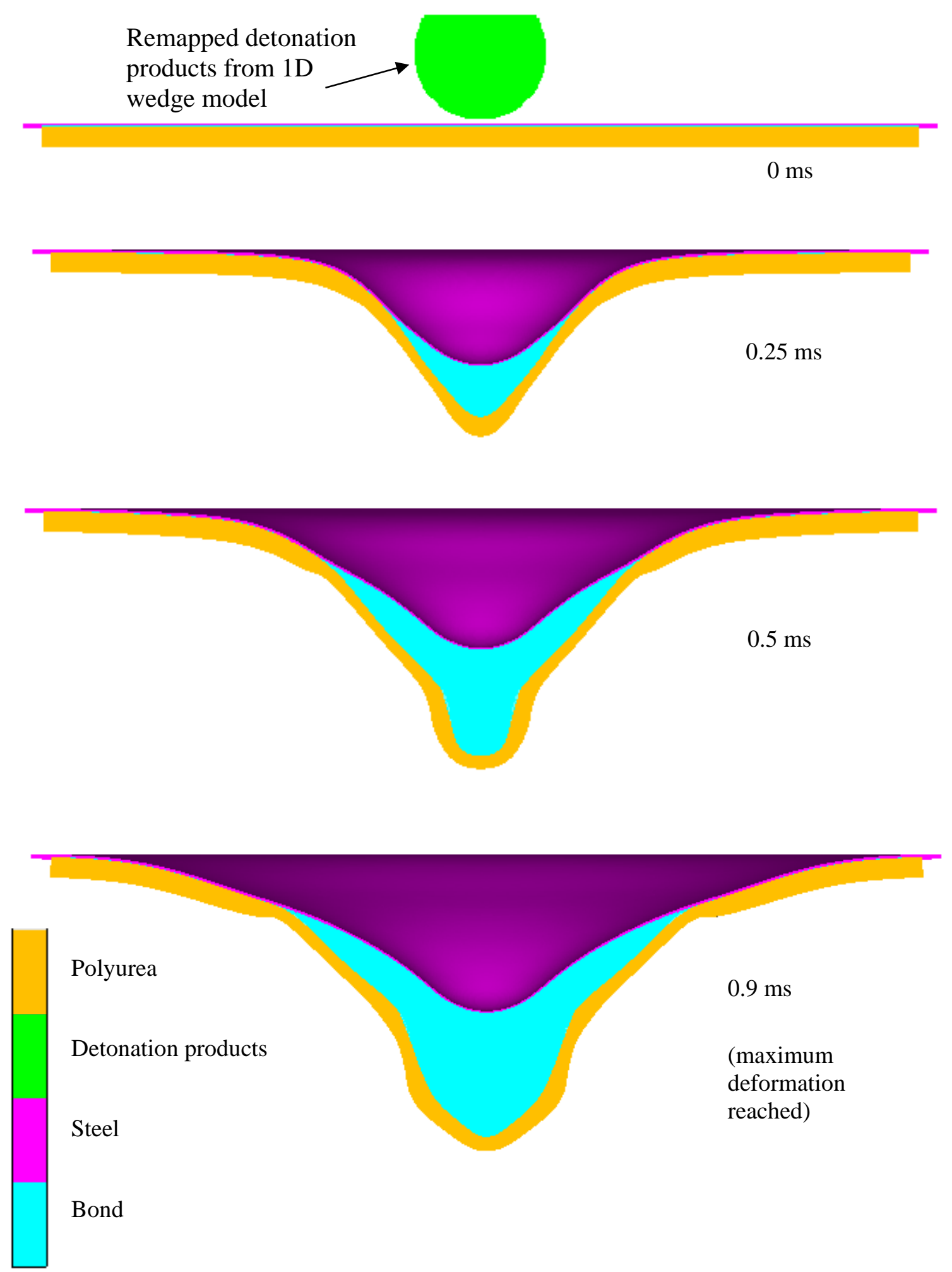

Figure 5.9 Polyurea de-bonding for the $4 \mathrm{~mm}$ steel plate with a $15.7 \mathrm{~mm}$ thick polyurea coating. The light blue region represents the void caused by local de-bonding. 

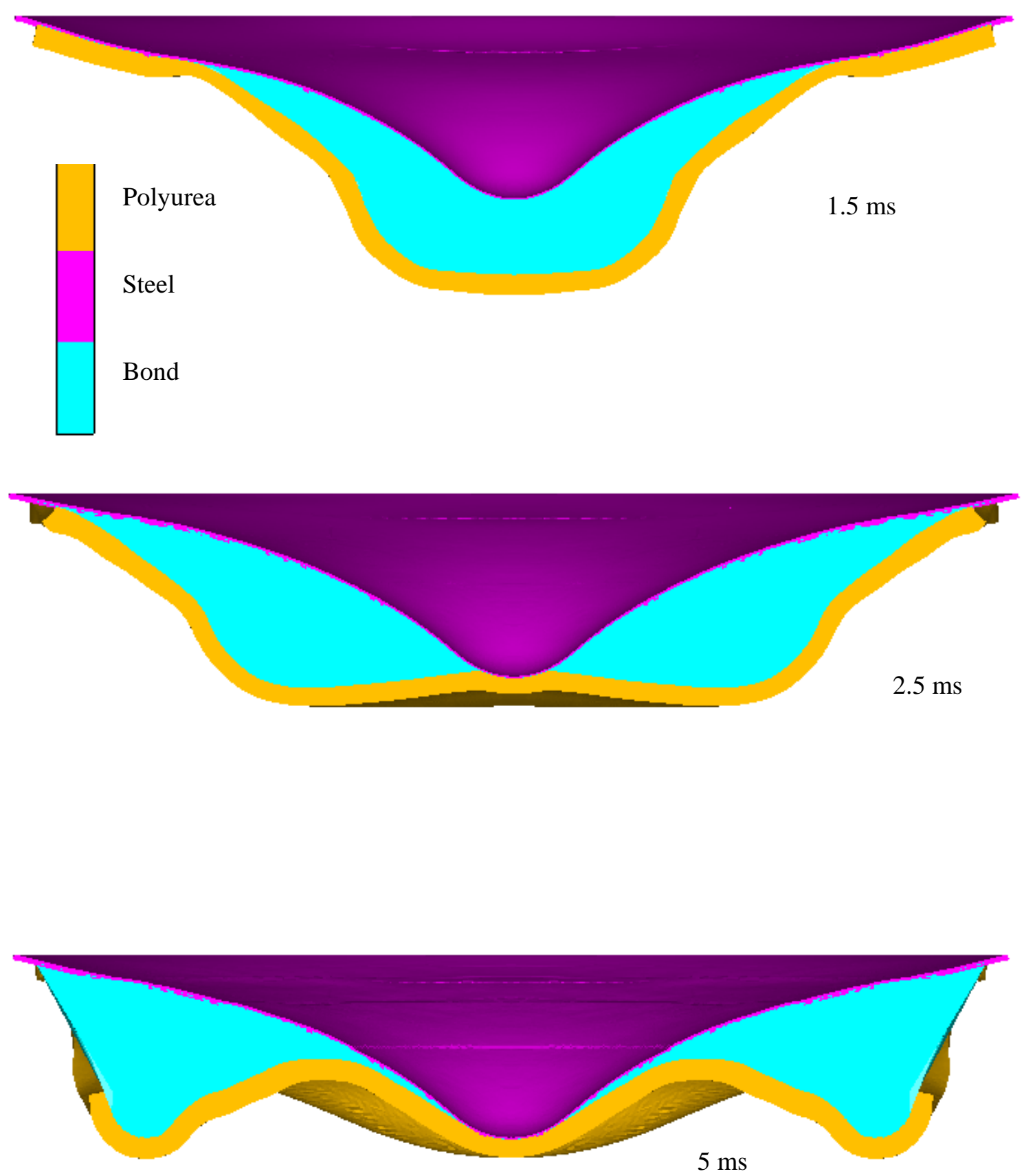

Figure 5.10 Polyurea de-bonding (cont.) for the $4 \mathrm{~mm}$ steel plate with a $15.7 \mathrm{~mm}$ thick polyurea coating. The bond is shown to fail across the width of the plate at the sectioned position (from $2.5 \mathrm{~ms}$ ). 
Figure 5.11 shows the element distortion of the de-bonded polyurea for the $5 \mathrm{~mm}$ plate with the $7.7 \mathrm{~mm}$ thick coating, as it reaches its peak transient deformation. Although there is some stretching of the elements, they appear to be adequate for capturing the polyurea deformation without excessive distortion or mesh entanglement.

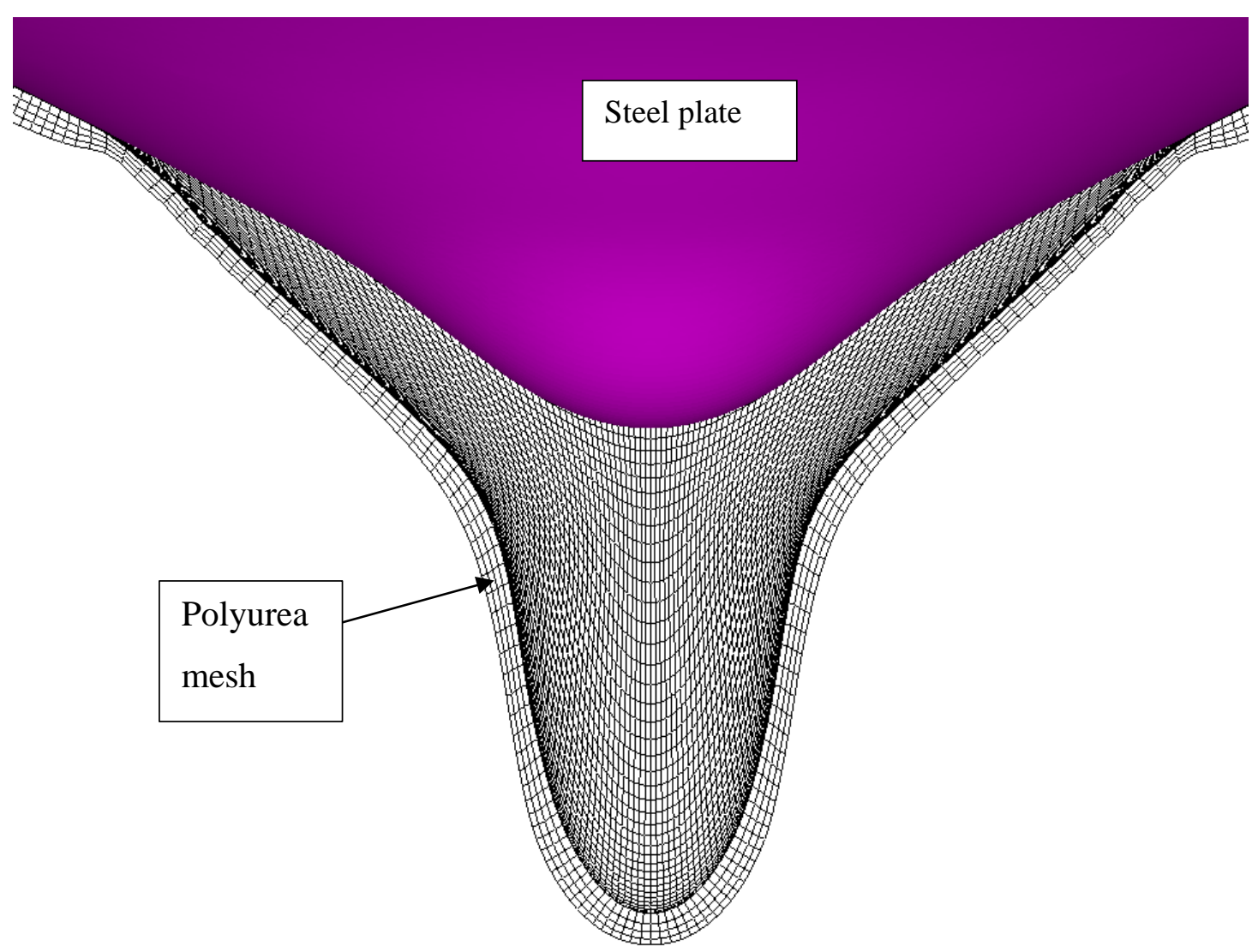

Figure 5.11 Element distortion of the de-bonded polyurea for the $5 \mathrm{~mm}$ plate with the $7.7 \mathrm{~mm}$ thick coating.

The numerical models of de-bonding allowed the time at which de-bonding occurred to be estimated, as this was not measured in the experiment. Initial de-bonding for the plate with the $7.7 \mathrm{~mm}$ thick polyurea coating was seen at the plate centre $12 \mu$ s after detonation. By the time the loading was at least 95\% complete (at $0.3 \mathrm{~ms}$ after detonation), de-bonding had extended to a radius of $81 \mathrm{~mm}$, which is close to one third of the final de-bonded radius. This means that in terms of area, approximately $90 \%$ of the de-bonding occurred after the loading phase was complete. For the $15.7 \mathrm{~mm}$ coating, similar results were seen, with over $90 \%$ of the de-bonding 
occurring after the loading phase. This result confirms that the polyurea coatings will have contributed to the response of the plates to the blast loading prior to de-bonding.

\subsubsection{Numerical modelling results and comparison with the experiments}

Table 5.2 shows the peak residual deformations determined using the numerical models, with yield stresses from Table 5.1. Excellent agreement is shown for the bare steel plates, decreasing for the polyurea-coated plates. This may be due to factors such as the simplification of the bond model and the material modelling of the polyurea. However, errors of $9 \%$ and $11 \%$ are relatively low given the complexity of the problem being simulated.

Table 5.2 Residual deformation results of the numerical modelling.

\begin{tabular}{|c|c|c|c|c|c|}
\hline Plate & $\begin{array}{l}\text { Target } \\
\text { description }\end{array}$ & $\begin{array}{l}\text { Yield of plate } \\
\text { model (MPa) }\end{array}$ & $\begin{array}{l}\text { Average } \\
\text { experimental } \\
\text { deformation } \\
(\mathrm{mm})\end{array}$ & $\begin{array}{l}\text { Numerical } \\
\text { deformation } \\
(\mathrm{mm})\end{array}$ & $\begin{array}{l}\text { Percentage } \\
\text { error (\%) }\end{array}$ \\
\hline V1, V2 & $\begin{array}{l}4 \mathrm{~mm} \text { bare } \\
\text { steel }\end{array}$ & 366 & 120.1 & 121.3 & 1 \\
\hline $\begin{array}{l}\text { B1, B2, } \\
\text { B3, B4 }\end{array}$ & $\begin{array}{l}6 \mathrm{~mm} \text { bare } \\
\text { steel }\end{array}$ & 446 & 115.1 & 112.8 & 2 \\
\hline $\begin{array}{l}\text { C1,C2, } \\
\text { C3 }\end{array}$ & $\begin{array}{l}5 \text { mm steel } \\
+ \text { coating }\end{array}$ & 400 & 124.1 & 134.7 & 9 \\
\hline $\begin{array}{l}\text { D1, D2, } \\
\text { D3 }\end{array}$ & $\begin{array}{l}4 \text { mm steel } \\
+ \text { coating }\end{array}$ & 402 & 135.7 & 149.2 & 11 \\
\hline
\end{tabular}

Figures 5.12 to 5.14 compare the numerical and experimental residual deformation plate profiles of the $6 \mathrm{~mm}$ bare plate, the $5 \mathrm{~mm}$ plate with a polyurea coating and the $4 \mathrm{~mm}$ plate with a polyurea coating, respectively. In all three cases the residual 
deformation profile as determined through numerical modelling matches closely with the experimentally measured profile.

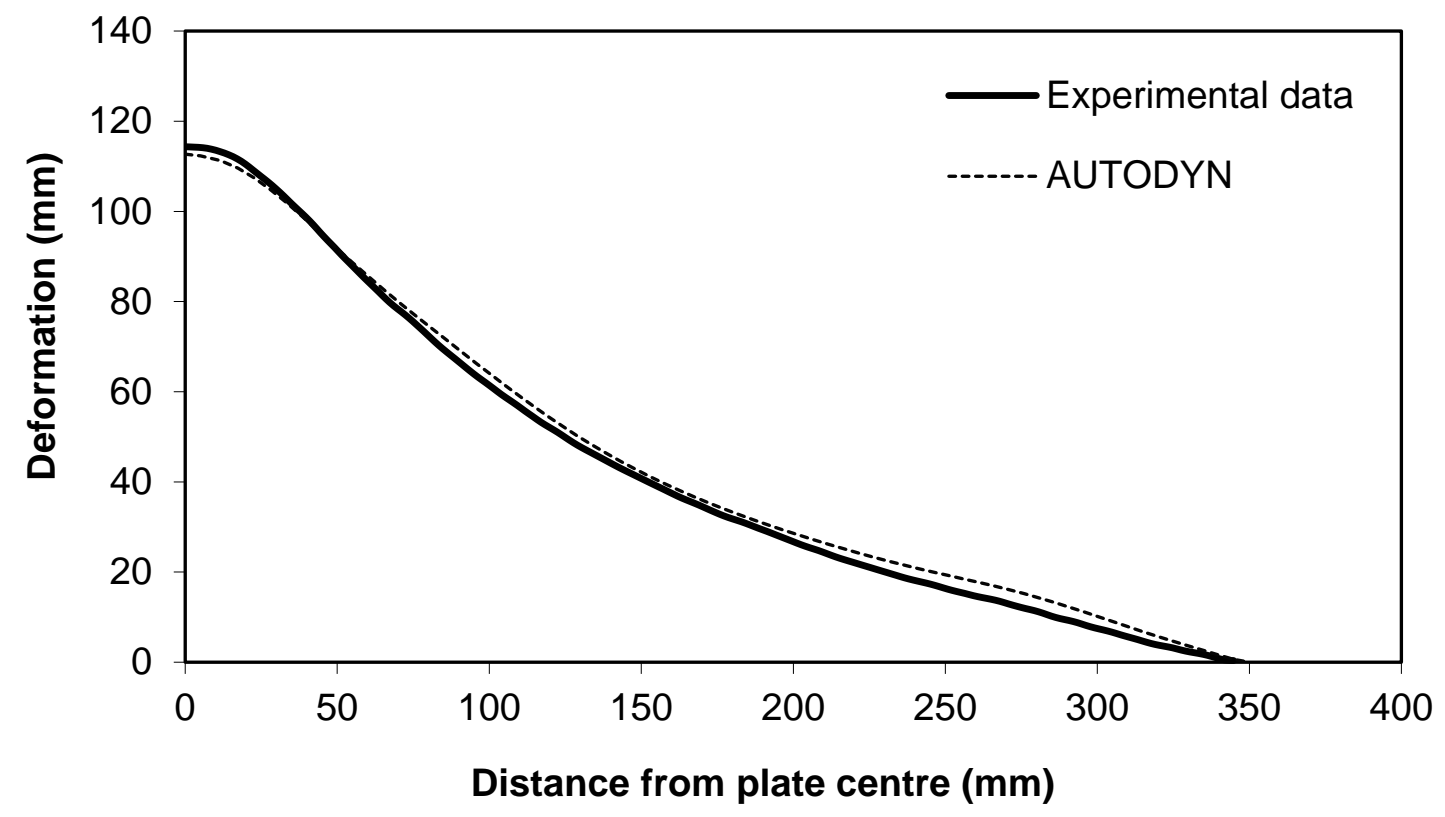

Figure 5.12 Comparison of the experimental plate deformation profile and numerical modelling result for the uncoated $6 \mathrm{~mm}$ steel plate (Plate B4). 


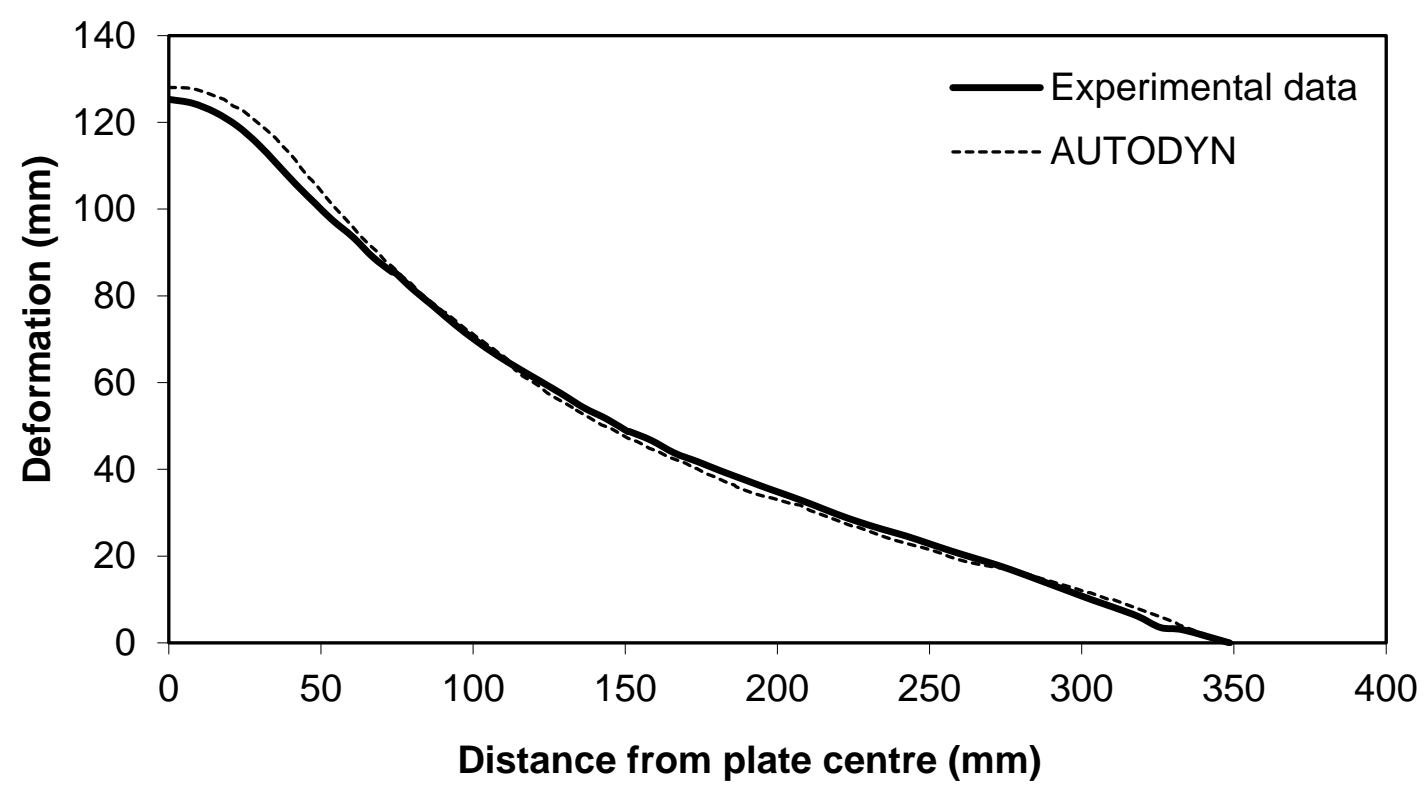

Figure 5.13 Comparison of the experimental plate deformation profile and numerical modelling result for the $5 \mathrm{~mm}$ steel plate with a polyurea coating (Plate C3).

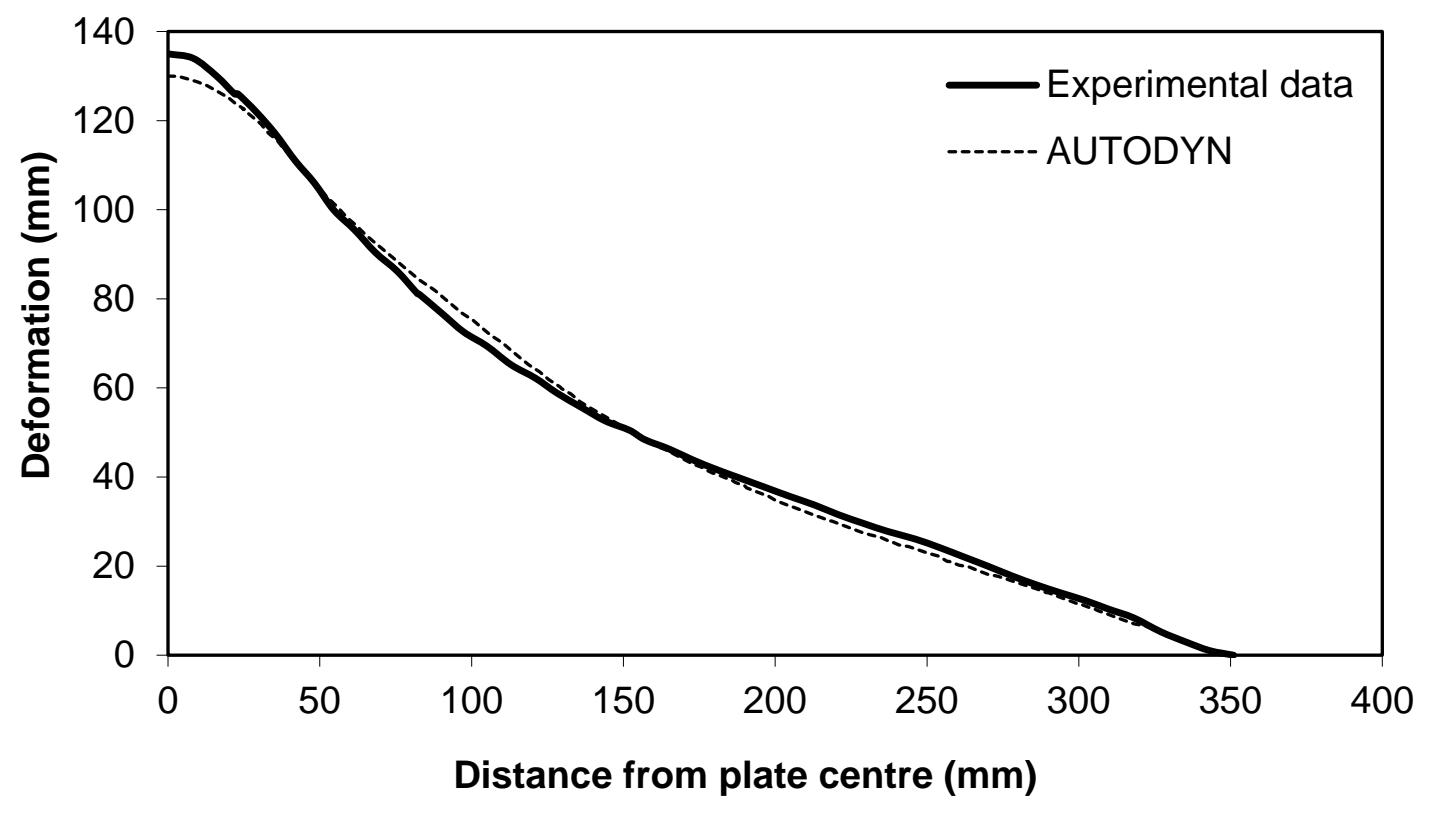

Figure 5.14 Comparison of the experimental plate deformation profile and numerical modelling result for the $4 \mathrm{~mm}$ steel plate with a polyurea coating (Plate D3). 
Figure 5.15 shows the numerical predictions for the dynamic response of the bare steel plate compared with the experimental results extracted from the high speed video footage. It can be seen that although the experimental and numerical results matched closely in terms of the residual deformation, the numerical results do not precisely predict the peak transient plate deformation or the frequency of the oscillations seen at the plate centre. The peak transient deformation of the bare steel plate as observed in the video footage was $127 \mathrm{~mm}$, occurring at $1.8 \mathrm{~ms}$ after detonation, whereas the peak transient deformation of the numerical model was only $114 \mathrm{~mm}$, occurring at $1.4 \mathrm{~ms}$. A likely cause for this discrepancy is that the fixed boundaries in the numerical model were an oversimplification of the experimental boundaries. Although efforts were made to clamp the boundaries in the experiment, some slippage at the plate boundaries occurred, possibly enabling greater dynamic deformation at the plate centre. The difference in the frequency of the plate oscillations after the loading phase is complete is also believed to result from the different boundary conditions. In the numerical model, the perfectly clamped boundary condition would have resulted in an increase in plate boundary stiffness compared to the experimental bolted boundary. This increase in boundary stiffness would result in a higher natural frequency of the plate, hence we observe the shortened period of oscillation at the latter times (from $~ 1 \mathrm{~ms}$ ) seen in Figure 5.15. 


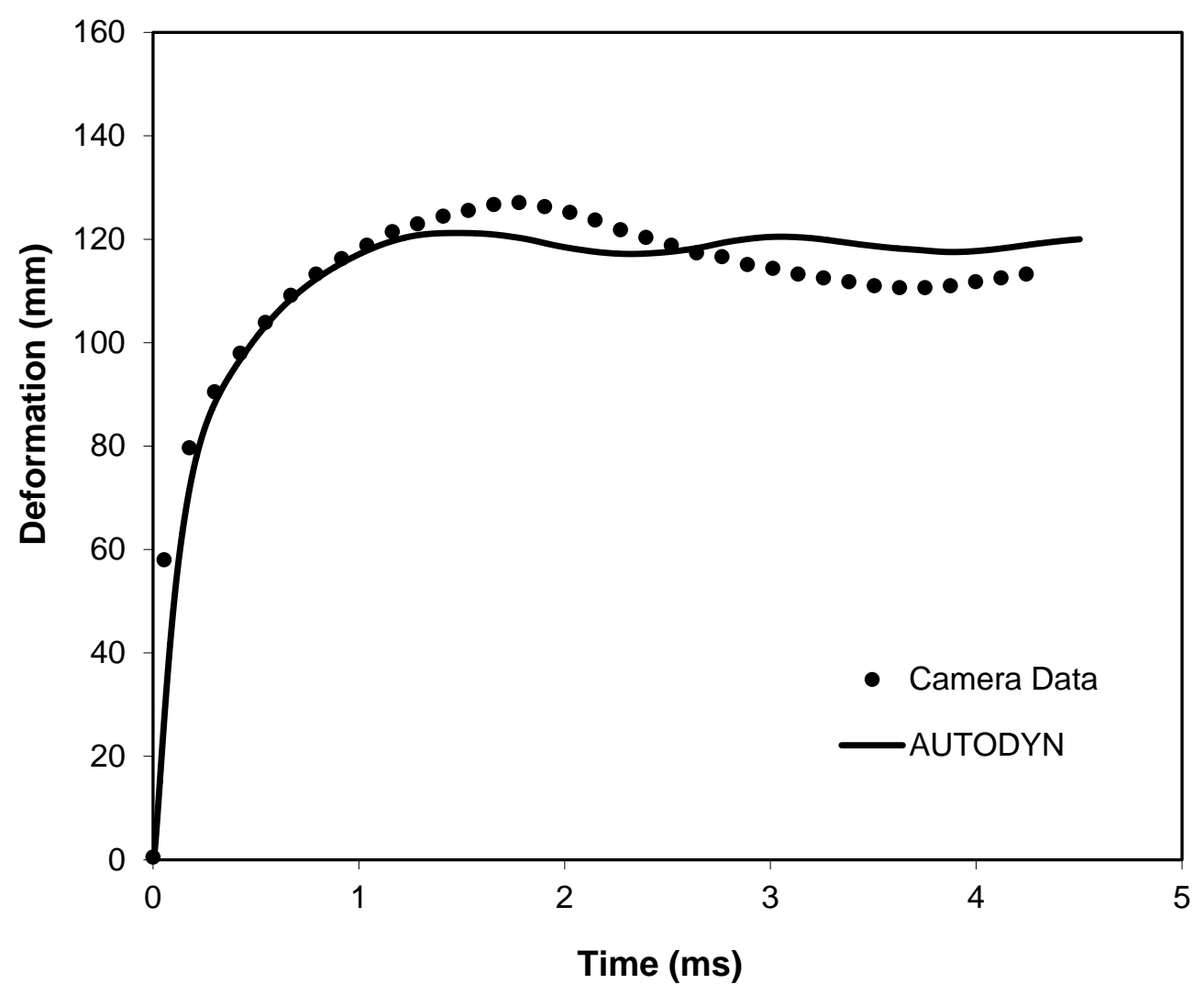

Figure 5.15 Comparison of high speed video data with AUTODYN predictions for the deformation at the plate centre of an uncoated $6 \mathrm{~mm}$ steel plate (B4) under blast loading. 
Figure 5.16 shows a comparison between the numerical and experimental results for the transient deformations for the three different plate configurations. The experimental measurements are from the high speed camera footage used during Events 8, 9 and 10. Note that the missing data points between 0.5 and $1.5 \mathrm{~ms}$ for Event 9 were due to the leading edge of the polyurea moving outside the camera field of view. The results for the bare plate shown by the squares and thin solid line are the same results as those shown in Figure 5.15, and are shown here again for comparison with the other plates. Observing the results for the $5 \mathrm{~mm}$ plate with the thin coating, denoted by the triangles and the thick solid line, it can be seen that the numerical results under-predict the transient deformation and the elongation of the polyurea as it de-bonds and moves away from the plate. The peak transient deformation of the polyurea in the numerical model was $197 \mathrm{~mm}$, compared with $263 \mathrm{~mm}$ calculated previously from the high speed video footage. Also, in the numerical model the polyurea begins moving back towards the plate at an earlier time ( $0.7 \mathrm{~ms}$ compared with $1 \mathrm{~ms}$ ) and returns to rest against the plate at an earlier time than seen in the experiment (1.5 ms compared with $2.3 \mathrm{~ms}$ ). The same can be seen for the $4 \mathrm{~mm}$ plate with the thick coating, which reaches a maximum transient deformation of $227 \mathrm{~mm}$ in the numerical model compared with $266 \mathrm{~mm}$ as measured from the experimental high speed video footage. The thick coating in the numerical model also comes back to rest against the plate at an earlier time of $2.3 \mathrm{~ms}$ compared with $3 \mathrm{~ms}$ in the experiment. 


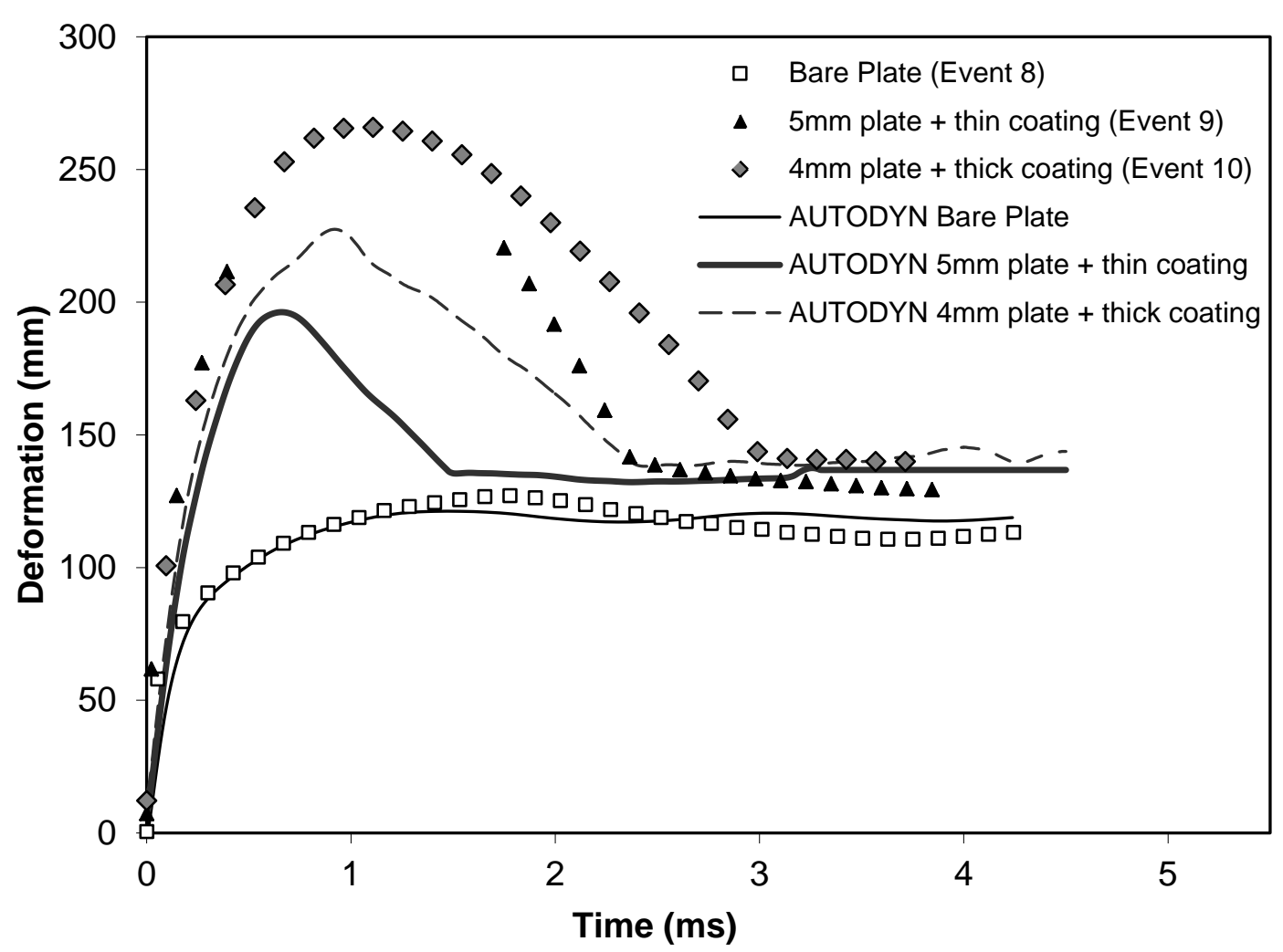

Figure 5.16 Comparison of experimental and numerical results for deformation at the plate centre.

Inspection of the numerical models showed that although the strain rates reached instantaneous values over $1000 \mathrm{~s}^{-1}$, the average strain rates seen in the polyurea were in the order of $10 \mathrm{~s}^{-1}$. Figure 5.17 shows the fitted data (previous shown in Chapter 3 , Figure 3.11) with a marker indicating a typical strain and strain rate from the numerical model. The typical strain rates seen in the model are an order of magnitude lower than the strain rate to which Mooney-Rivlin parameters were fit, which was over $300 \mathrm{~s}^{-1}$. At low strain rates, the Mooney-Rivlin fit significantly over-predicts the typical experimental stresses, by approximately 50\% at $14 \mathrm{~s}^{-1}$ and $100 \%$ strain. This means that the modelled polyurea will absorb more energy as it deforms, compared to the experimental polyurea which will flow with lower resistance to larger strains. This difference is likely to have contributed to the under-predictions of peak deformations seen in Figure 5.16. 


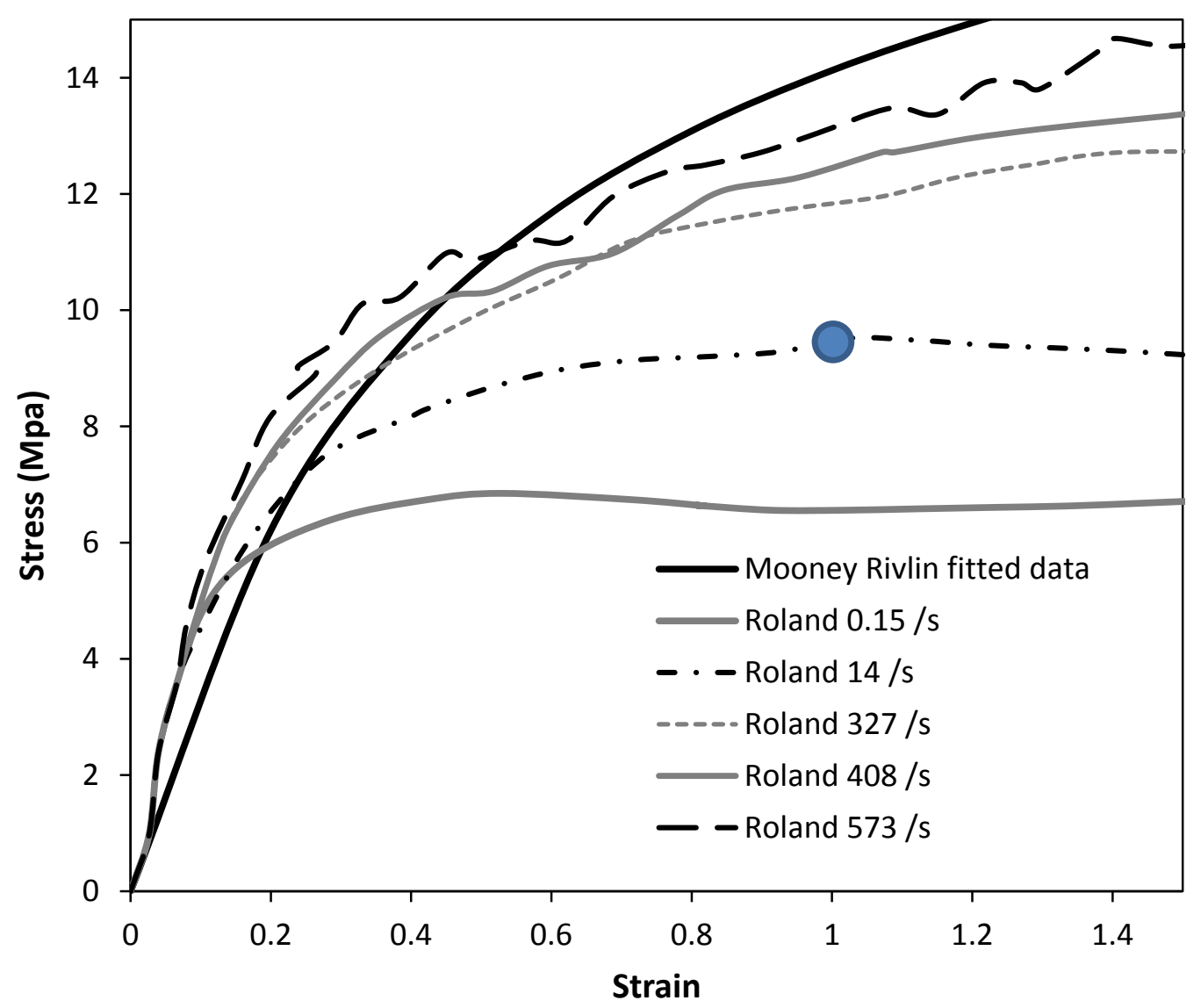

Figure 5.17 Mooney-Rivlin fit to polyurea stress-strain data from Roland et al. (2007), with the marker showing the approximate peak strain and strain rate from the numerical model.

It is also noted that the polyurea tested by Roland et al. (2007), from which the Mooney-Rivlin parameters were derived, had a stoichiometry of $96 \%$, compared to $105 \%$ for the polyurea used in the experiments in Chapter 4. Roland et al. (2007) showed that polyureas with higher stoichiometries carry less stress than those with lower stoichiometries (Figure 2.6). Thus the polyurea modelled in the simulations could be expected to predict less transient deformation than the polyurea material used in the experiments.

The choice of the third Mooney-Rivlin constant $d$ may have also contributed to the discrepancies between the numerical and experimental results. This constant is related to the bulk modulus, $\kappa$, by $d=2 / \kappa$. The bulk modulus cannot be accurately determined through uniaxial tensile testing. For the numerical simulations, a bulk modulus of 
$\kappa=4.98$ was used. This was the only value found in literature for a polyurea with a stoichiometry of $105 \%$. This value was determined through confined compression tests conducted by Amirkhizi et al. (2006). Other values have been reported in literature for different stoichiometries. Chakkarapani et al. (2006) found a bulk modulus of 2.1 GPa for cast polyurea through confined compression testing, however the stoichiometry of the polyurea is not reported. Roland and Casalini (2007) used dielectric spectroscopy to measure the local segmental relaxation times for the soft segments of a polyurea as a function of temperature and pressure, giving a bulk modulus of $2.57 \mathrm{GPa}$ for a polyurea with a stoichiometry of $96 \%$. To investigate this further, the effect of varying the bulk modulus within the values reported in literature is studied numerically later in this chapter.

\subsection{Refinement of the Mooney-Rivlin constants}

In order to develop a more accurate material model for polyurea, tensile testing of the polyurea used in this thesis was conducted at varying strain rates. This allowed new constants $C_{01}$ and $C_{10}$ to be fit to the Mooney-Rivlin material model. Testing was conducted at higher strain rates as well as those seen in the models to provide data which may be useful in future studies.

\subsubsection{Tensile testing}

The testing was conducted following a procedure used by Raman (2011) and with the assistance of Jinghan Lu from Melbourne University. Dynamic high strain rate tests were conducted under uniaxial tension, using an Instron 8800 servo-hydraulic universal testing machine at the Solid Mechanics and Dynamics Laboratory, Swinburne University of Technology. A quasi-static test with a strain rate of $0.01 \mathrm{~s}^{-1}$ was also conducted at the same laboratory, using an MTS servo-hydraulic tensile test machine, which has a maximum test velocity of $5 \mathrm{~ms}^{-1}$.

Tensile specimens were manufactured according to Figure 5.18. For the high strain rate tests, the samples were bolted to a steel extender to reduce the length of the polyurea test section. 

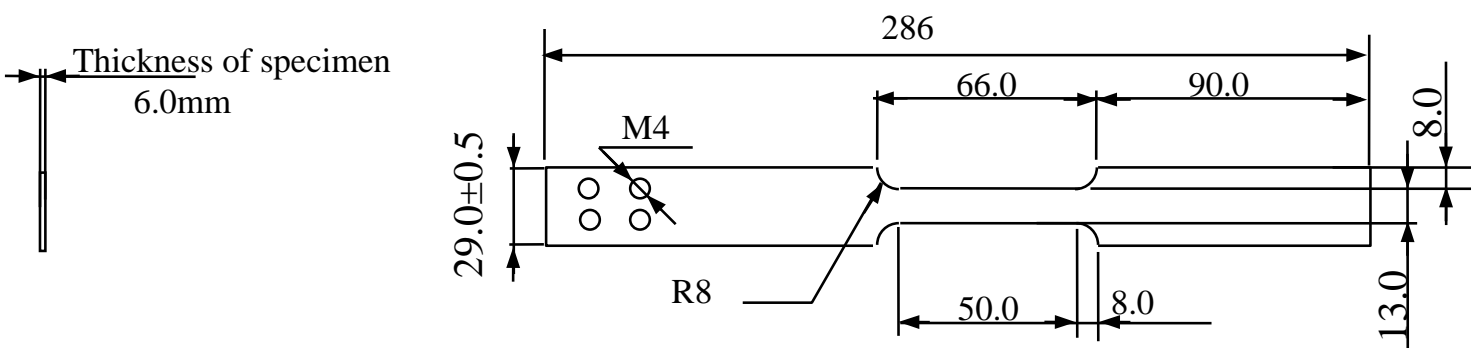

Figure 5.18 Tensile specimen dimensions (Lu, 2011).

An RTV silicone mould was used to form the polymer tensile specimens. The polyurea was poured into the mould and excess material was removed from the top. The coating was then allowed to cure at room temperature for a minimum of 24 hours before being removed from the mould and post-cured at $50^{\circ} \mathrm{C}$ for 24 hours in an oven (the same procedure used for the plate coatings). Figure 5.19 shows one of the specimens used for the tensile tests. Figure 5.20 shows one of the polyurea samples loaded in the machine before and after testing. Note that the polyurea was not tested to failure as it was beyond the scope of this research. The sample below did not fail before it reached its maximum extension.

Figure 5.19 Tensile specimens used for the initial tests. 

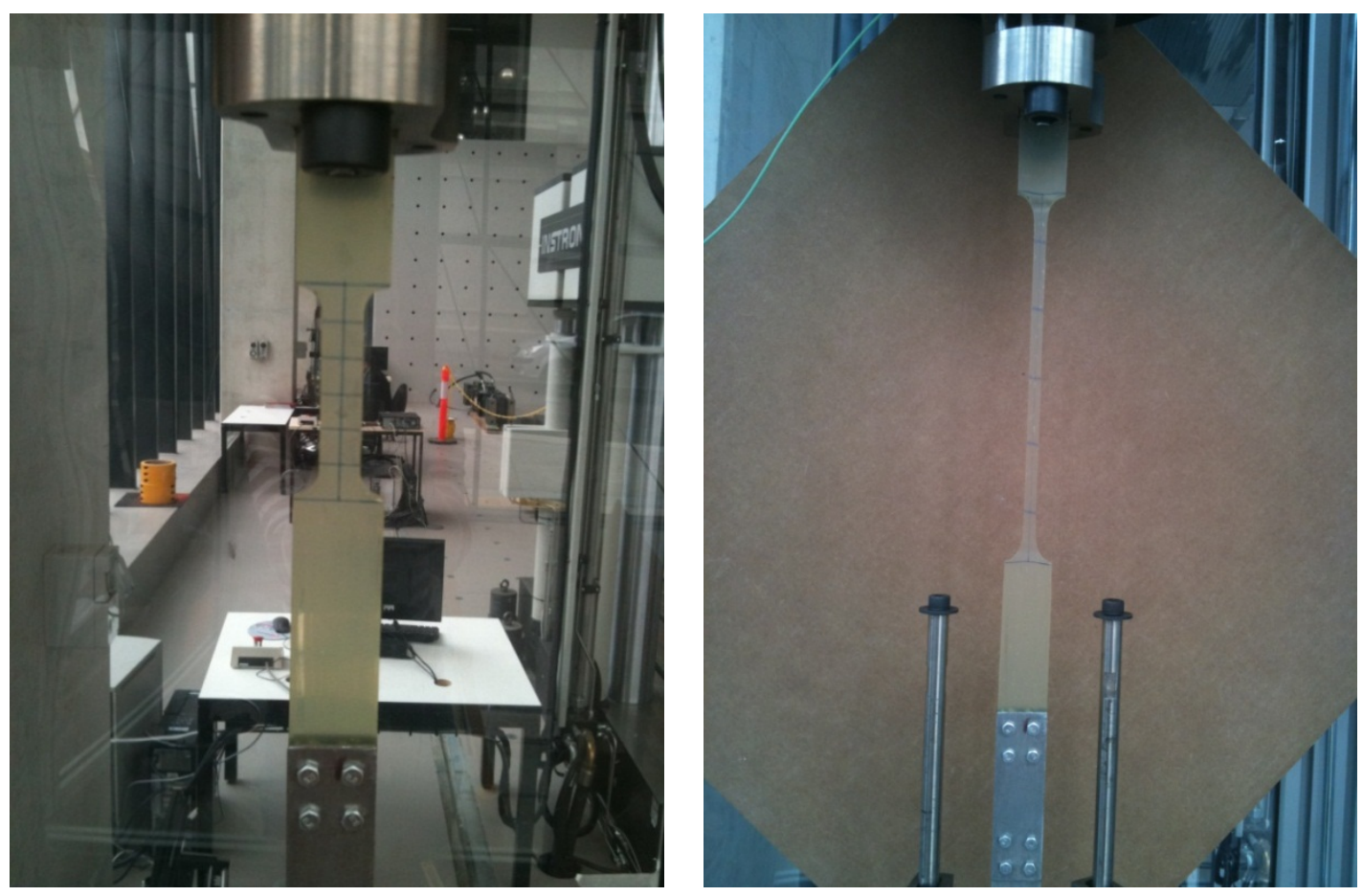

Figure 5.20 Tensile test specimen in the Instron 8800 test machine. The left image shows the specimen before testing, and the right hand image shows the specimen after testing.

Figure 5.21 shows the results of the dynamic high strain rate tensile tests of polyurea. Measurements were taken up to strains of $250 \%$, as this range is the most relevant to blast testing. Only a single curvature is observed (rather than with an inflection as seen in Figure 2.7), and the results generally showed a slight increase in stiffness with increasing strain rate. As the strain rate is increased, the material is also able to carry higher stresses, especially at higher strains. This is similar to the behaviour seen in high strain rate tensile tests of polyurea by Roland et al. (2007). 


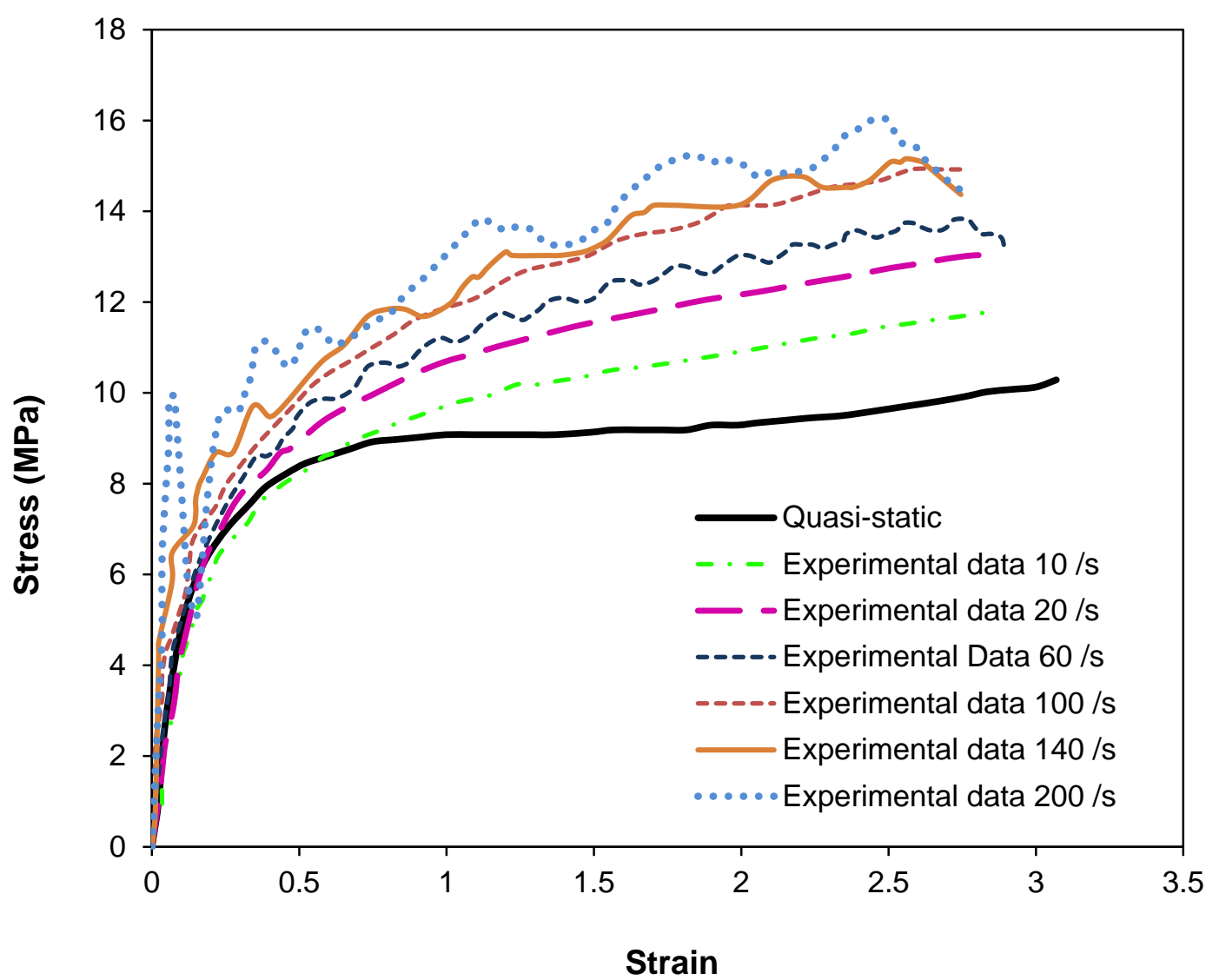

Figure 5.21 Results of quasi-static and dynamic tensile tests on polyurea.

\subsubsection{Fitting constants to the data}

Mooney-Rivlin constants were fit to the data recorded at $10 \mathrm{~s}^{-1}$, to better match the average strains found in the numerical models. In the literature review, it was found that for a Mooney-Rivlin hyperelastic material, plotting $(\sigma / 2)\left(\lambda-\lambda^{-2}\right)$ against $1 / \lambda$ yields a straight line, where $\lambda$ is the stretch ratio $(\lambda=1+\varepsilon)$. This was plotted for the polyurea tensile test results and a linear trend line was fitted to the data. Initially the entire dataset was used, with the curve fit shown in Figure 5.22. When stress was plotted against strain using the resulting constants $\left(C_{01}=-1461.1 \mathrm{kPa}\right.$ and $C_{10}=$ $9077.6 \mathrm{kPa}$ ), the resulting curve is shown in Figure 5.23. It is noted that units of $\mathrm{kPa}$ are required for the constants to maintain dimensional equality in Equation 2.10, however they are non-physical and thus can be negative. The quasi-static tensile test results have also been included in Figure 5.23, as these represent the lower bound for 
the stress-strain curve. Although the curve fits well at low strains $(<0.3)$, the fit is poor at strains above $\sim 2.5$.

To create a better fit at higher strains (as strains of up to around 1.0 are seen in the models), the data points below strains of 0.5 were removed from the curve fitting procedure. Figure 5.24 shows the new linear curve fit which resulted in constants $C_{01}=5568 \mathrm{kPa}$ and $C_{10}=43.9 \mathrm{kPa}$. Figure 5.25 shows the resulting stress-strain plot achieved when using these constants. The resulting curve fit was found to fit a wider range of strains, up to around 2.5. Strains in the previous numerical models using the original Mooney-Rivlin constants were found to be in the order of 1.0, and higher strains may be seen if the new material parameter set allows larger polyurea deformations. Hence the constants $C_{01}=5568 \mathrm{kPa}$ and $C_{10}=43.9 \mathrm{kPa}$ were chosen to use for further modelling. It was recognised that the initial stiffness in Figure 5.25 is slightly lower in the fitted data compared to the experiments; however the MooneyRivlin model could not be used to fit the data closely at both low and high strains. It was decided that the high strain region was more important to model accurately due to the large deformations of the polyurea seen after de-bonding. However, the effect of increasing the material model stiffness was identified as a parameter to be investigated further in parametric studies, which are discussed later in this chapter. 
Chapter 5. Numerical Simulations and Parametric Studies.

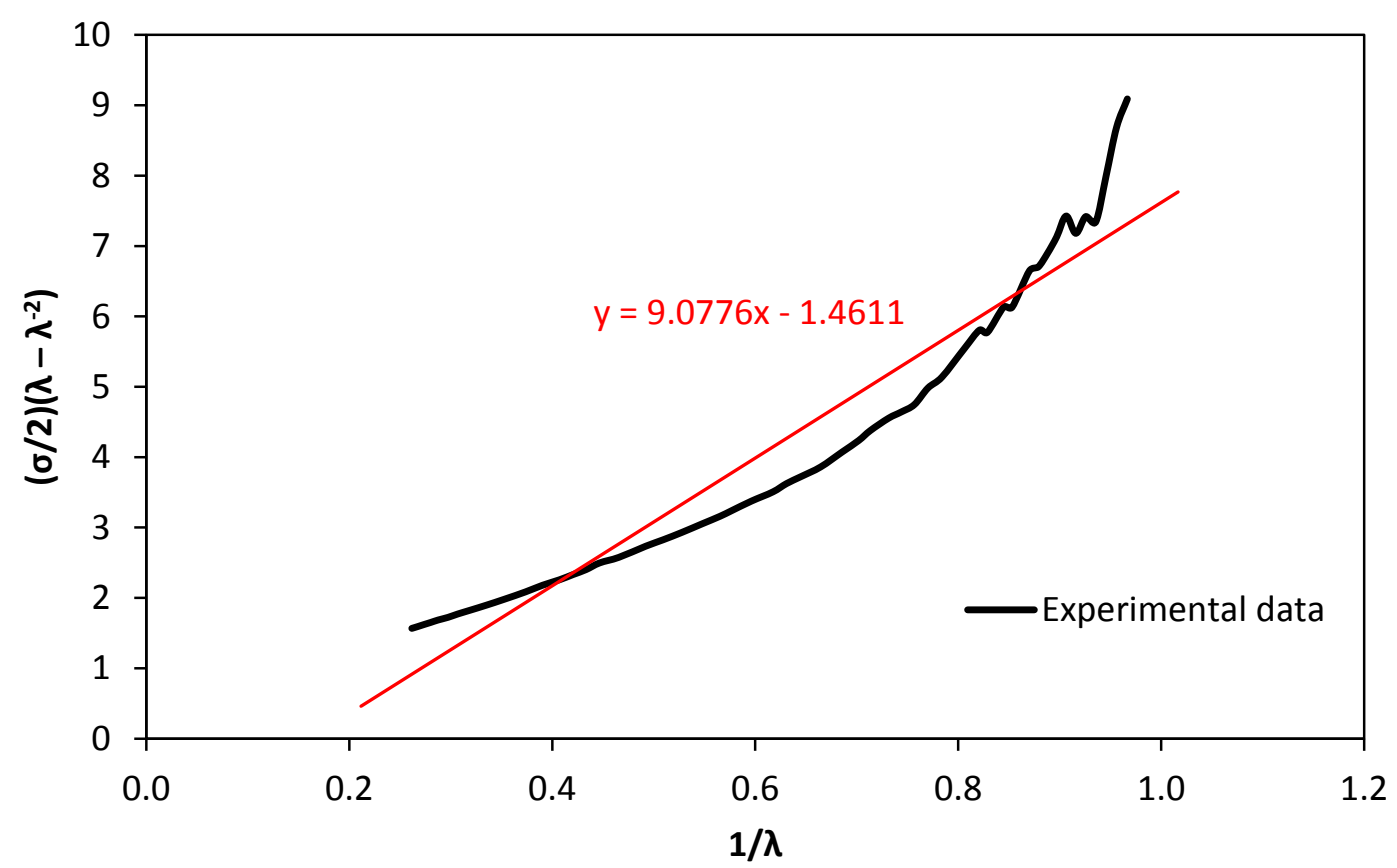

Figure 5.22 Linear curve fit to experimental data from dynamic high strain rate tensile tests at $10 \mathrm{~s}^{-1}$. 
Chapter 5. Numerical Simulations and Parametric Studies.

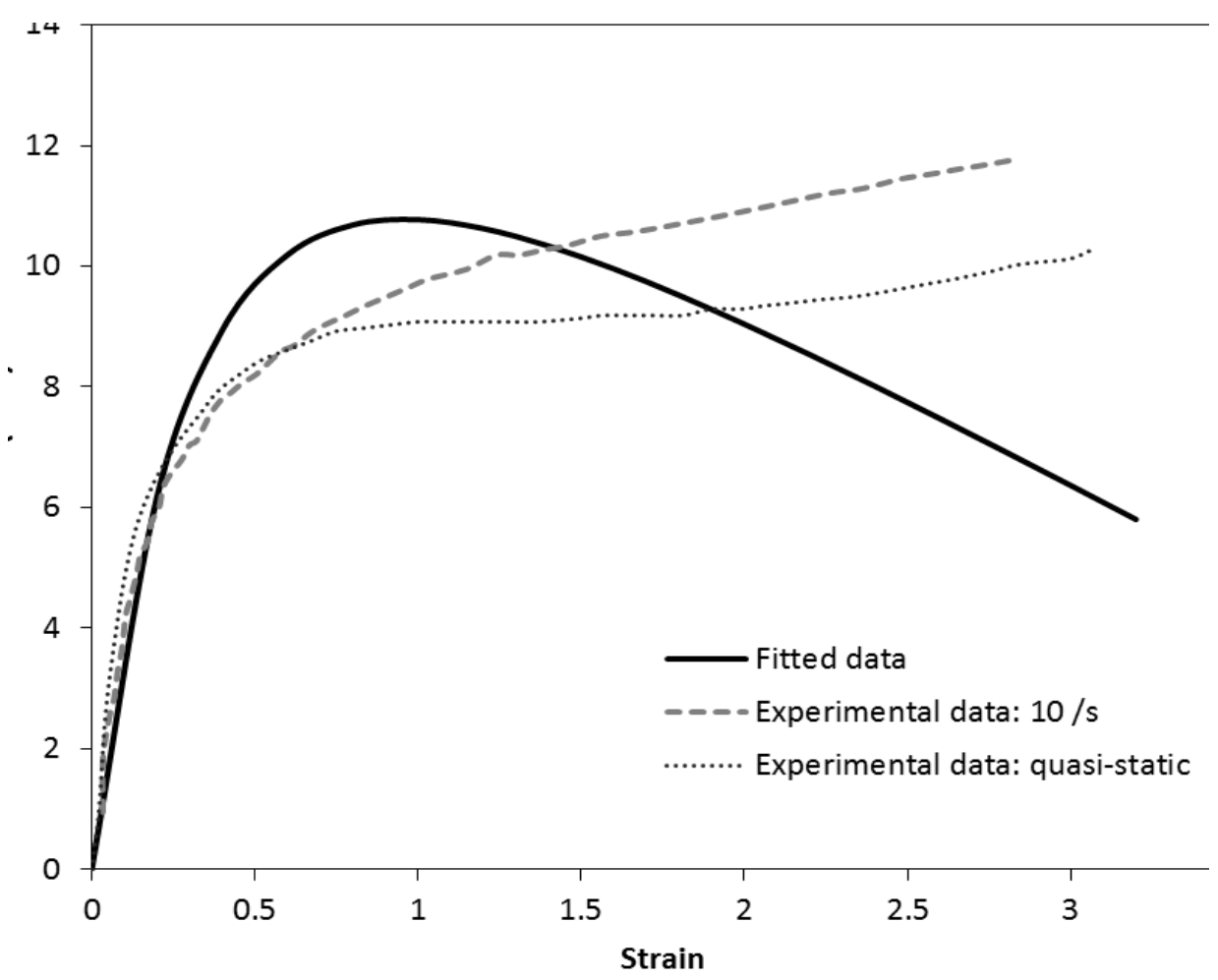

Figure 5.23 2-parameter Mooney-Rivlin curve fit to high strain rate tensile test results at $10 \mathrm{~s}^{-1}$ using constants $C_{01}=-1461.1 \mathrm{kPa}$ and $C_{10}=$ $9077.6 \mathrm{kPa}$.

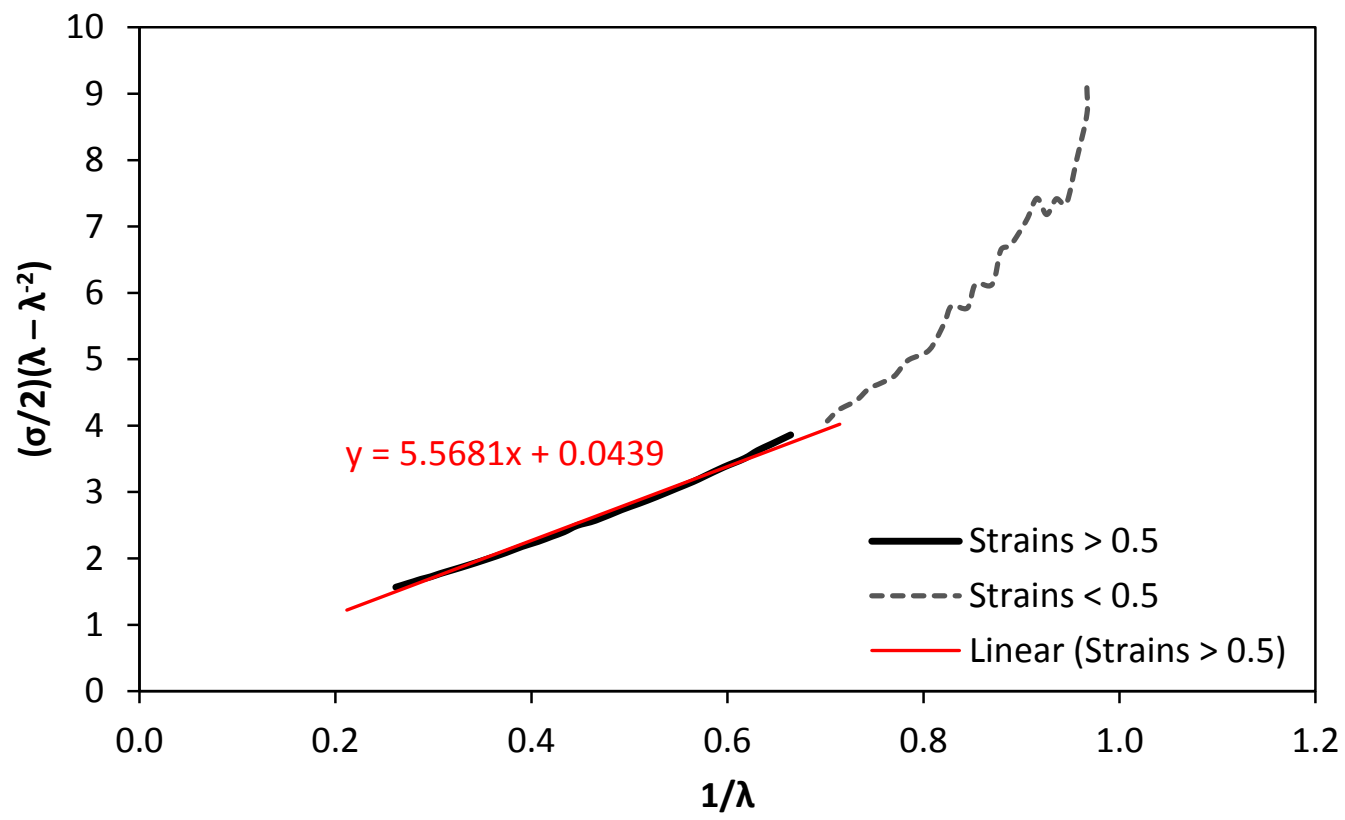

Figure 5.24 Linear curve fit to experimental data from dynamic high strain rate tensile tests at $10 \mathrm{~s}^{-1}$ with data points removed for strains less than 0.5 . 


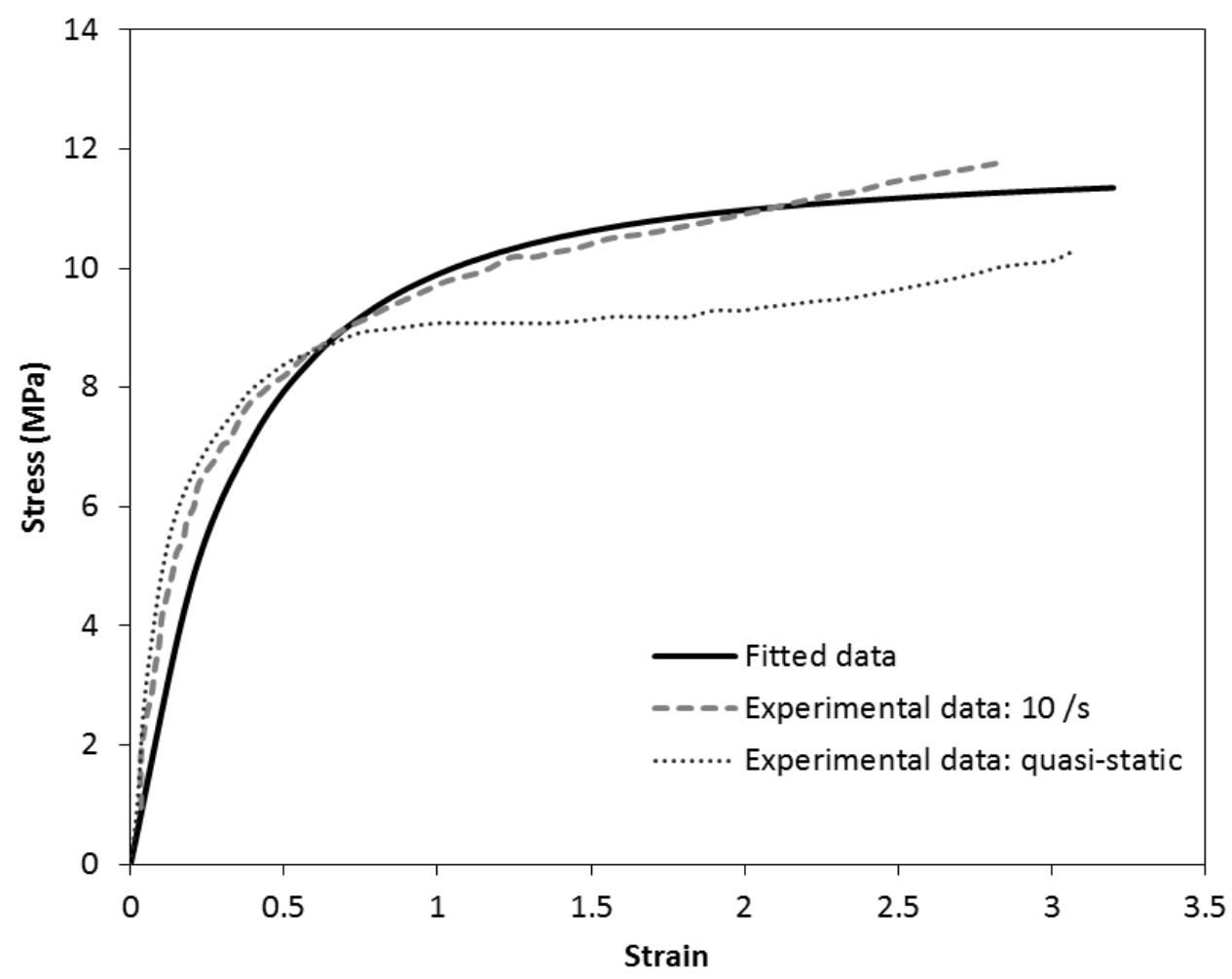

Figure 5.25 2-parameter Mooney-Rivlin curve fit to high strain rate tensile test results at $10 \mathrm{~s}^{-1}$ using constants $C_{01}=5568 \mathrm{kPa}$ and $C_{10}=43.9 \mathrm{kPa}$.

\subsection{Numerical modelling of the experiments with new material model constants}

The numerical models described in Section 5.2 were re-run with the new MooneyRivlin material model constants, $C_{01}=5568 \mathrm{kPa}$ and $C_{10}=43.9 \mathrm{kPa}$. In order to match the radius of the de-bonded area, the bond strength was reduced to $80 \mathrm{MPa}$. Figure 5.26 and Figure 5.27 show the results for the residual deformation profiles compared with the experimental results. The deformation profiles from the numerical models match the experimental results very well; however the peak residual deformation was slightly higher for the $5 \mathrm{~mm}$ plate with the thin polyurea coating and slightly lower for the $4 \mathrm{~mm}$ plate with the thick polyurea coating. The curve fits are comparably good with those found using the original Mooney-Rivlin constants (Figure 5.12 and Figure 5.13). The strains were well within the tested range (up to $250 \%$ ), with maximum strains in the simulated polyurea of around $130 \%$. 
Chapter 5. Numerical Simulations and Parametric Studies.

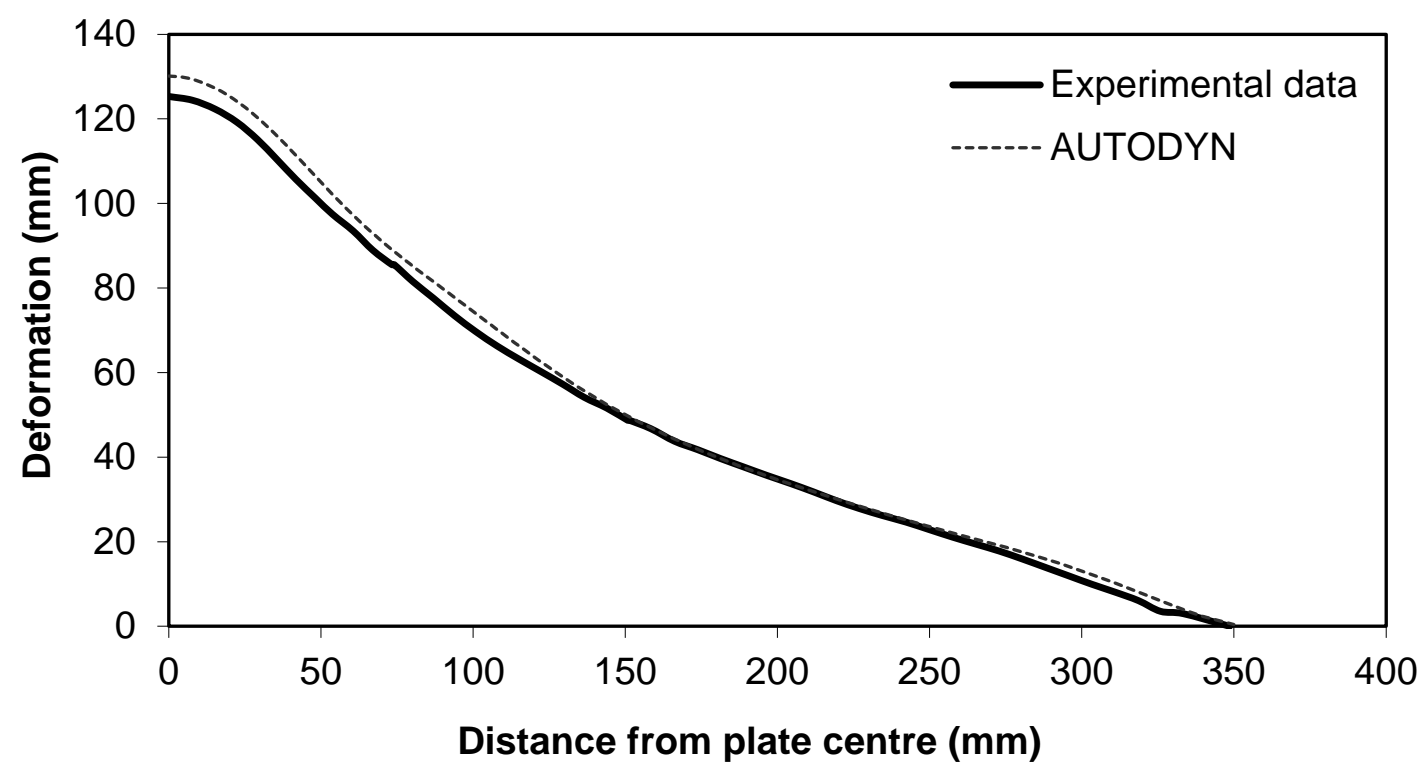

Figure 5.26 Comparison of the experimental plate deformation profile and numerical modelling result for the $5 \mathrm{~mm}$ steel plate with a polyurea coating (Plate C3), using new Mooney-Rivlin constants.

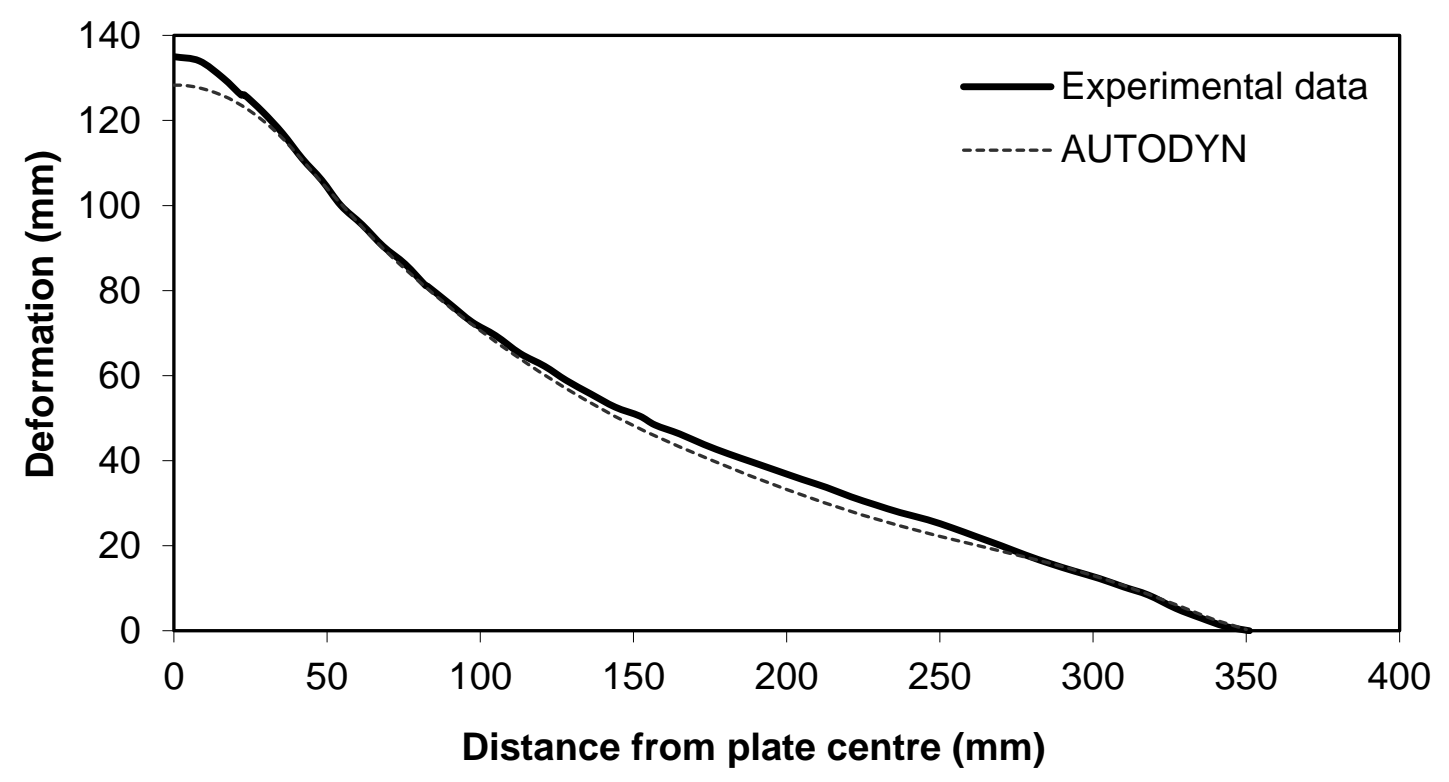

Figure 5.27 Comparison of the experimental plate deformation profile and numerical modelling result for the $4 \mathrm{~mm}$ steel plate with a polyurea coating (Plate D3), using new Mooney-Rivlin constants. 
Figure 5.28 shows the numerical modelling results for transient deformation, using the new Mooney-Rivlin constants. Table 5.3 compares the values for peak transient and residual deformations. It can be seen in Figure 5.28 that the numerical results for the $4 \mathrm{~mm}$ plate with the thick polyurea coating match the experimental results closely, particularly in comparison with previous results shown in Figure 5.16. The peak numerical transient deformation was within $1 \%$ of the experimental value, compared to a previous under-prediction of $15 \%$. The results for the $5 \mathrm{~mm}$ polyurea-coated plate were also improved when using the new constants; however the peak transient deformation was still slightly under-predicted. In this case, the peak numerical transient deformation was around $12 \%$ less than the experimental value, compared with a $25 \%$ under-prediction using the original constants. This could be partially due to the fitting of the polyurea material model which varies in accuracy over different strains and strain rates (Figure 5.25). In the lower strain region (strains less than 0.5), an over-prediction of transient deformation would be expected as the stiffness is lower in the material model than measured in the tensile tests. This is true for both the quasistatic and $10 \mathrm{~s}^{-1}$ responses, which are similar up to around 0.6 strain. Above this strain the model over-predicts the stresses for the quasi-static response, which would lead to reduced deformations.

Another contributing factor to the discrepancies in Figure 5.28 may be the simplification of the bond failure using a principal stress criterion. The difference may also arise from experimental variations such as slightly different bond strengths for different targets. Figure 5.6, although using a different material model, suggests that for the bond strengths seen here ( $\sim 0 \mathrm{MPa})$, a slight change in bond strength can affect the radius of the de-bonded area, which may in turn have an effect on the transient deformation. The diameters of the de-bonded area of the three $5 \mathrm{~mm}$ polyurea-coated plates (C1, C2 and C3) were measured after the experiments as $480 \mathrm{~mm}, 505 \mathrm{~mm}$ and $500 \mathrm{~mm}$, respectively. The data points for the $5 \mathrm{~mm}$ polyureacoated plate shown in Figure 5.28 were found from high speed footage of a single plate, plate C3. The numerical model of the de-bonding assumed a radius of $247.5 \mathrm{~mm}$ (diameter $495 \mathrm{~mm}$ ), which could have led to a slight over-prediction of bond strength for plate C3, ultimately leading to lower predicted deformations. It is unclear whether this effect is significant enough to cause a measureable difference in peak transient deformation values, and it is likely that other factors contributed to this 
discrepancy. The effect of varying bond strength on transient deformation and de-bonding radius is explored numerically later in this chapter through parametric studies.

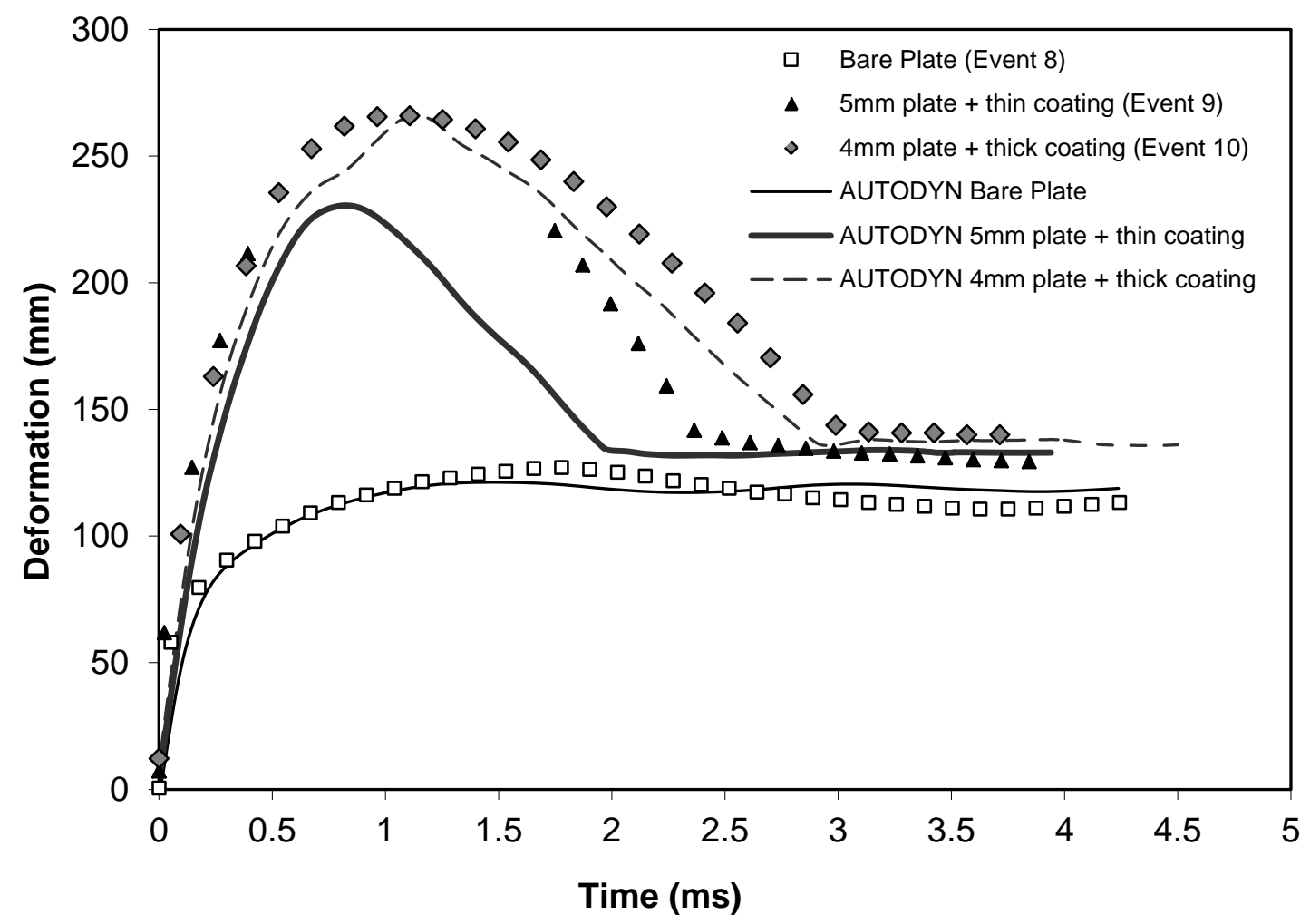

Figure 5.28 Comparison of experimental and numerical results for the deformation of the plate centre, using Mooney-Rivlin constants $C_{01}=5568 \mathrm{kPa}$ and $C_{10}=43.9 \mathrm{kPa}$. 
Table 5.3 Comparison of numerical modelling results using two different Mooney-Rivlin material models for polyurea.

\begin{tabular}{|c|c|c|}
\hline & Numerical m & \\
\hline $\begin{array}{l}\text { Experimental } \\
\text { result }\end{array}$ & $\begin{array}{l}\text { Original } \\
\text { (Nahshon, } \\
\text { NSWC-CD) }\end{array}$ & Updated model \\
\hline- & 875 & 43.9 \\
\hline - & 6321 & 5568 \\
\hline- & $4 \times 10^{-7}$ & $4 \times 10^{-7}$ \\
\hline- & 90 & 80 \\
\hline 263 & 197 & 230 \\
\hline 266 & 227 & 265 \\
\hline 124.1 & 131.8 & 130.1 \\
\hline 135.7 & 127.6 & 128.3 \\
\hline
\end{tabular}

\subsection{Parametric modelling studies}

Parametric studies were conducted in order to investigate how various properties affect plate response, with the aim of achieving potential improvements to the coating properties and the coating/plate configuration. Numerical models were created based on the models described in Section 5.4. 
Properties that were varied were:

- Bond strength

- Polyurea stiffness

- Polyurea bulk modulus

- Coating location (front, back and sandwich plate)

The effect of thickness on plate response to blast has already been studied experimentally. It was found that the peak transient deformation of the polyureacoated plates increased with thickness (or polyurea/steel mass ratio), as did the peak transient deformation. As the effect of thickness was already known, a single plate and coating thickness was selected for the parametric studies, being the $5 \mathrm{~mm}$ plate with a $7.7 \mathrm{~mm}$ coating. This was selected over the thicker coating as the de-bonded area was smaller, reducing the likelihood of complete de-bonding in the parametric simulations. Also, the run times to simulate this configuration were much shorter.

The performance measures for the parametric studies were the peak transient deformation and the de-bonded area. These are both important in vehicle or structural design. The peak transient deformation defines the potential damage zone behind the plate, and the de-bonded area will affect whether or not the polyurea will completely de-bond to form a secondary projectile, which is particularly important if the coatings are used on small components. The residual deformation was not considered here because although important in the model validation, it is not considered to be the preferred parameter to measure of the blast performance of the plate.

\subsubsection{Variations in bond strength}

The strength of the bond between the polyurea coating and the plate was identified earlier as a factor which may influence the response of the polyurea-coated plate to blast loading. The bond strength used to model the experiments was set to $80 \mathrm{MPa}$. In this parametric study, the effect of increasing the bond strength is studied by multiplying the bond strength by factors of 1.5 and 2, resulting in bond strengths of $120 \mathrm{MPa}$ and $160 \mathrm{MPa}$. In addition, the effect of a smaller change in bond strength was investigated, as this was identified in Section 5.4 as a possible source of error in 
some of the models. Bond strengths of $70 \mathrm{MPa}$ and $90 \mathrm{MPa}$ were used for this part of the study

Figure 5.29 and Figure 5.30 show numerical simulation results which demonstrate how the bond strength affects the peak transient deformation of the polyurea and the de-bonded area, respectively. It can be seen in Figure 5.29 that as the bond strength is increased, the peak transient deformation of the polyurea decreases. This is related to the de-bonded area, which decreases with increasing bond strength, Figure 5.30. Stronger bonds allow a larger area of the polyurea to remain attached to the plate during the blast loading, reducing the area of polyurea available for hyperelastic deformation, thus reducing the peak transient deformation. The decrease however is minimal; with a doubling of the bond strength from 80 to $160 \mathrm{MPa}$ decreasing the transient deformation by only $4.6 \%$.

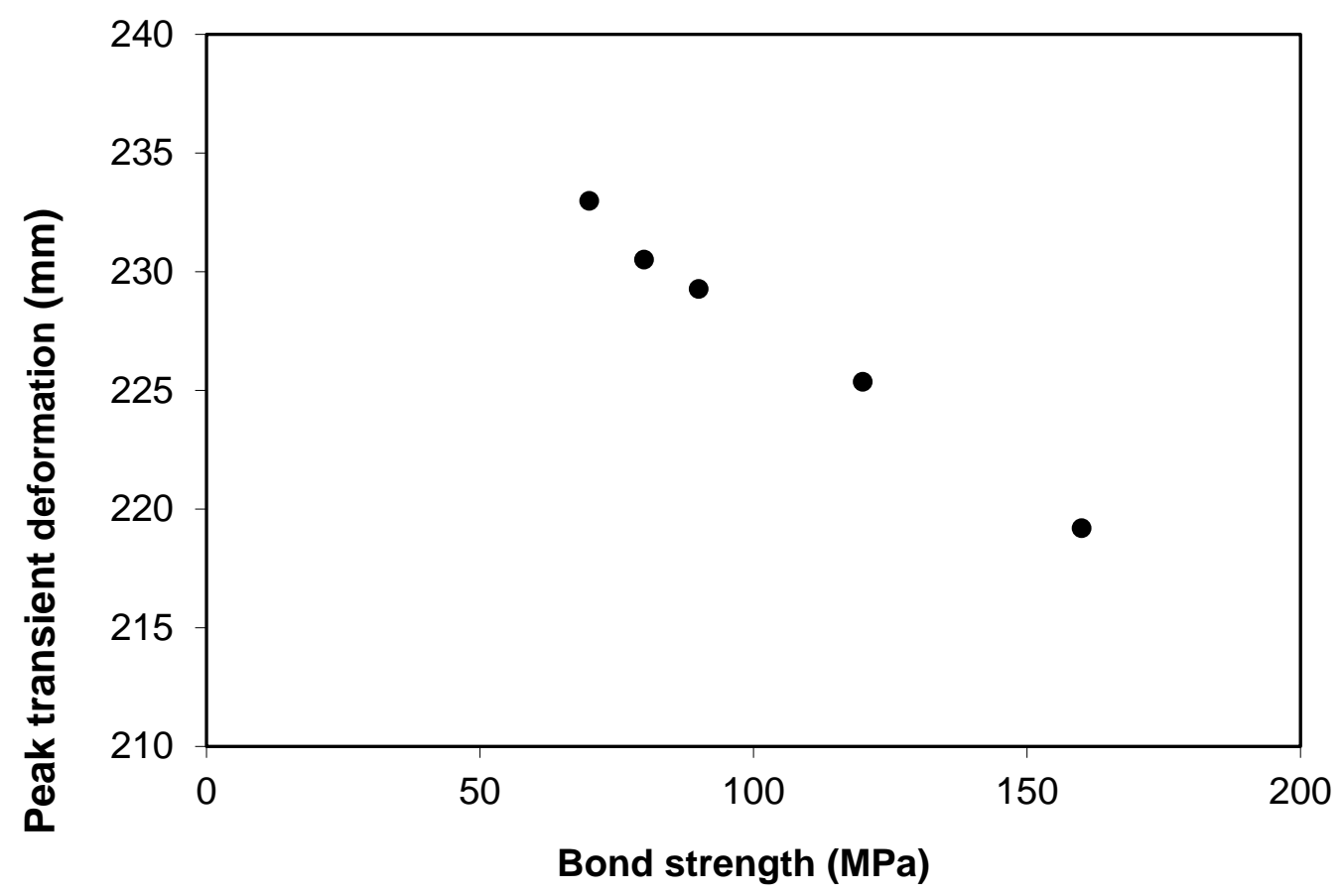

Figure 5.29 Effect of bond strength on the peak transient deformation of the polyurea coating. 


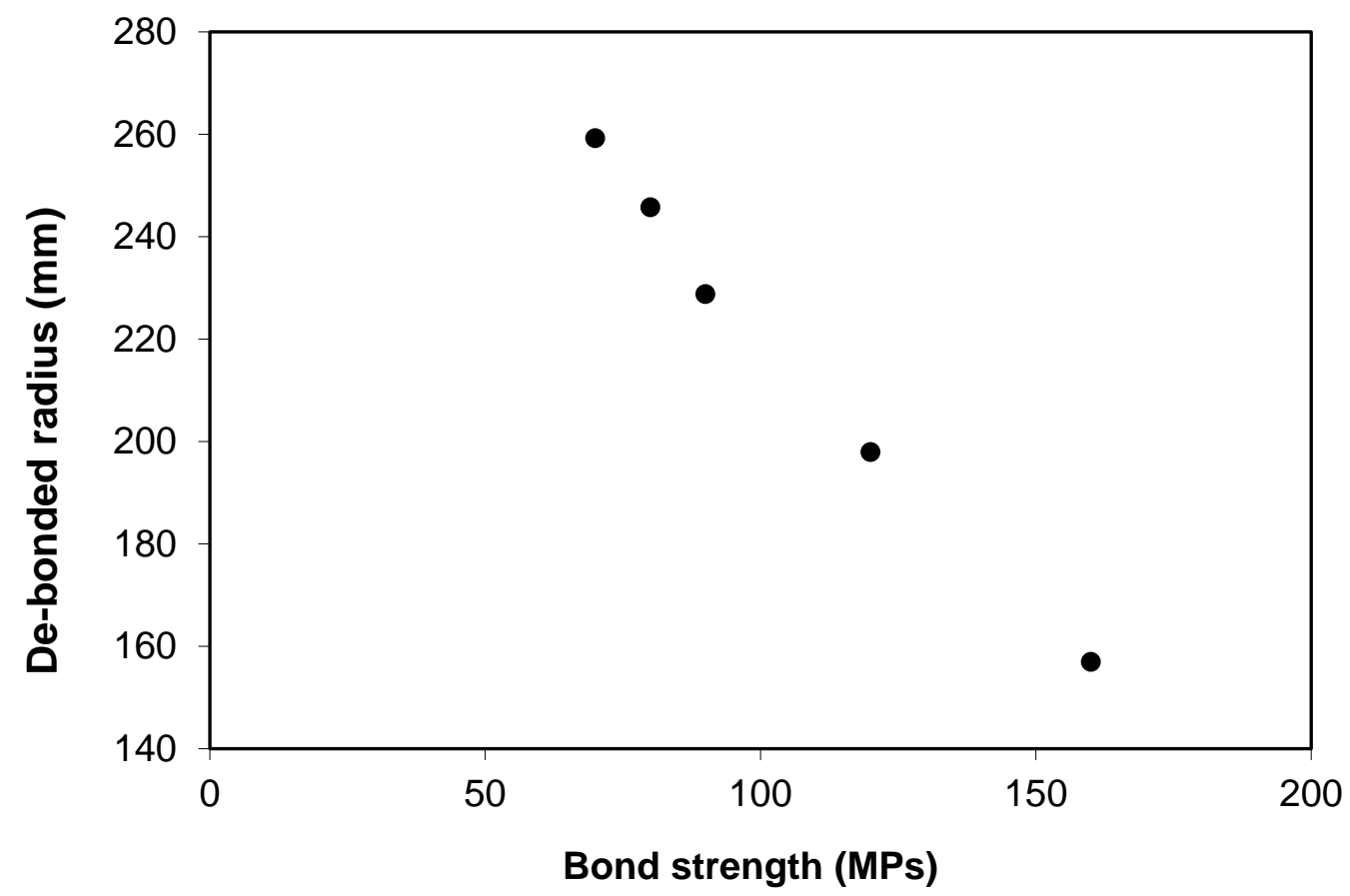

Figure 5.30 Effect of bond strength on the radius of the polyurea de-bonded area.

When considering the effect of a $10 \mathrm{MPa}$ change in bond strength, the resulting difference in the radius of the de-bonded area was found to be $16.9 \mathrm{~mm}(6.9 \%)$ when the bond strength is increased, or $13.5 \mathrm{~mm}$ (5.5\%) when the bond strength is decreased. This gives an average of around $15 \mathrm{~mm}$ difference. Given that the debonded radii in the experiments were $247.5 \pm 7.5 \mathrm{~mm}$, it is estimated that the bond strength in the experiments would have been $80 \mathrm{MPa} \pm 5 \mathrm{MPa}$. It was also found that the effect of a $10 \mathrm{MPa}$ difference in bond strength on peak transient deformation is small (less than $3 \mathrm{~mm}$ ), therefore the effect of a $5 \mathrm{MPa}$ difference will be even less. Thus the variation in bond strength is not sufficient to explain the $17 \mathrm{~mm}$ difference in peak transient deformation between the experimental and numerical results for the $5 \mathrm{~mm}$ plate with the $7.7 \mathrm{~mm}$ coating. The variation may instead be due to slight differences in the polyurea properties between the experiments, or the simplification of the bond failure model.

The simulation of bond failure here was simplified by assuming a principal stress failure criterion. In reality, the failure of the bond is expected to be more complex as it is subjected to compressive, tensile and shear forces as the plate is loaded and the 
coating de-bonds. There is also likely to be strain-rate discrepancies, as well as inconsistencies in the quality of the bond on the experimental plates. Further investigation of more realistic methods to model the bond between the plate and the polyurea may be considered in future studies.

\subsubsection{Variations in polyurea stiffness}

The initial stiffness of the numerical model for the polyurea coating can be found from the Mooney-Rivlin material model constants as $E=6\left(C_{01}+C_{10}\right)$. Using this relation, the initial stiffness of the material model used in Section 5.4 is $33.7 \mathrm{MPa}$. Figure 5.31 shows how changing the constants $C_{01}$ or $C_{10}$ can be used to change the initial stiffness (in this example by a factor of two). It can be seen that this affects the shape of the stress-strain curve. As the stiffness is increased, so is the area under the curve (i.e. a measure of material toughness). This characteristic is inherent in the Mooney-Rivlin material model, thus the parametric studies were continued with this in consideration. 
Chapter 5. Numerical Simulations and Parametric Studies.

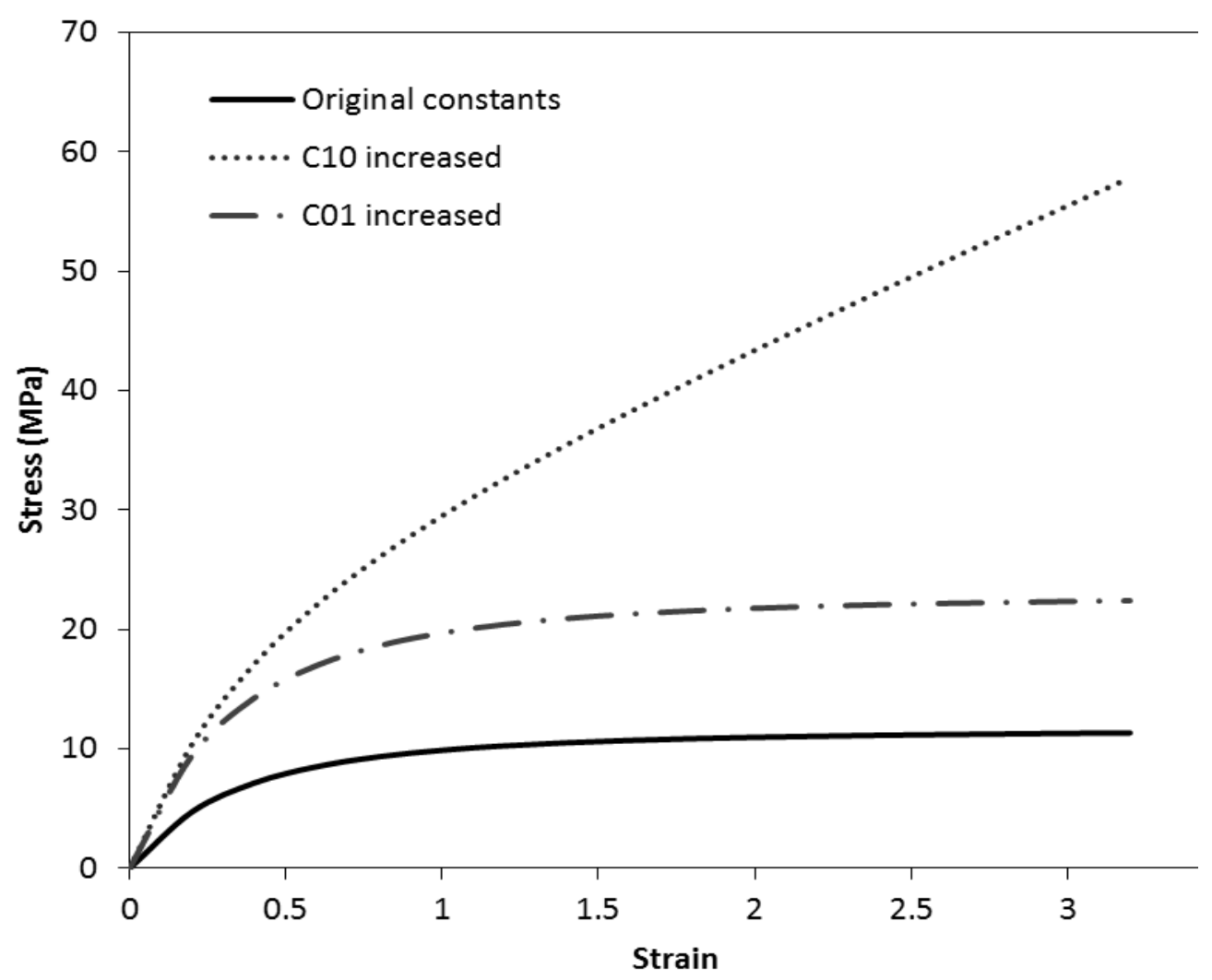

Figure 5.31 Effect on the stress strain curve from varying the Mooney-Rivlin material constants $C_{01}$ and $C_{10}$ to double the initial stiffness.

Figure 5.32 shows how the Mooney-Rivlin constants $C_{01}$ and $C_{10}$ were varied in this parametric study and their effect on the stress-strain curve. The stiffness, $E$, was first increased by factors of 1.5 and 2 by changing the constant $C_{01}$ (while keeping $C_{10}$ constant). In addition, the stiffness was increased by a factor 1.5 by changing $C_{10}$ (while keeping $C_{01}$ constant). 


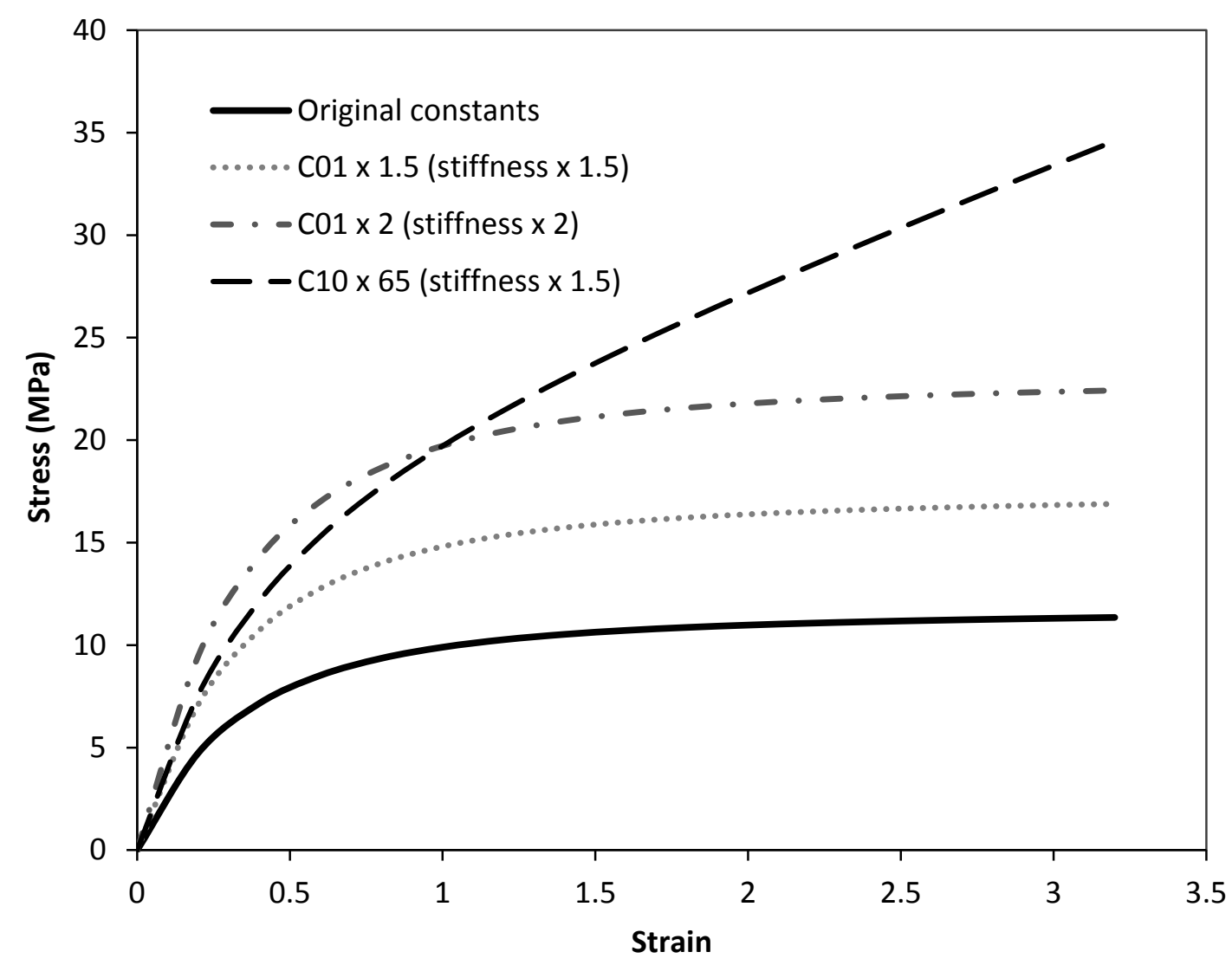

Figure 5.32 Stress strain plots using different Mooney-Rivlin constants.

Figure 5.33 shows how the stiffness affects the peak transient deformation of the polyurea. The plot shows that as $C_{01}$ is increased to increase the stiffness, the peak transient deformation of the polyurea is reduced. This suggests that increasing the stiffness (and the area under the stress-strain curve) is an effective way to reduce the transient polyurea deformation. As the area under the stress-strain curve represents the strain energy required to deform the polyurea, the curves with higher stiffness and larger enclosed areas require more energy input to strain the polyurea. Thus for the same load, the materials with higher stiffness will deform less. 


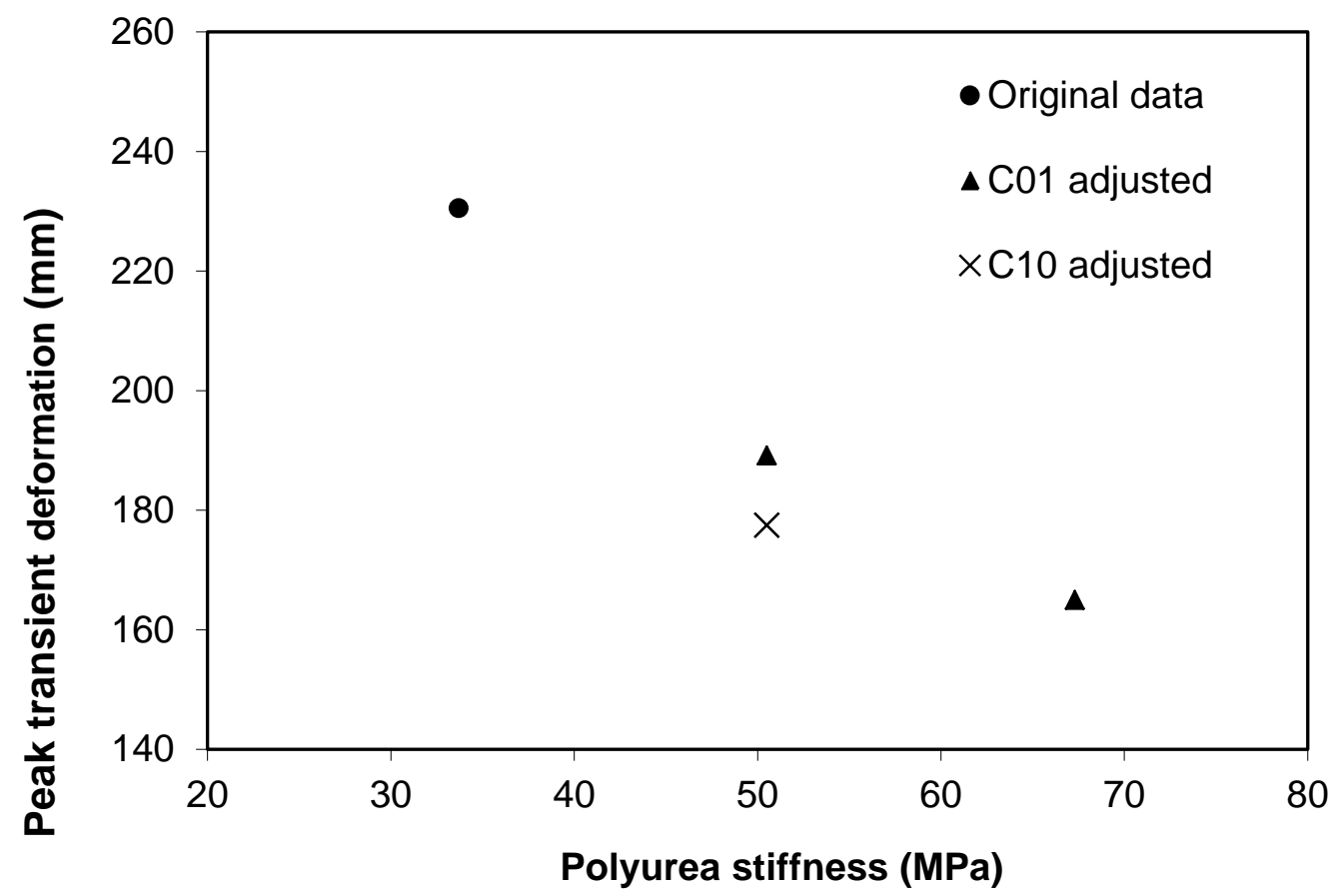

Figure 5.33 Effect of polyurea stiffness on the peak transient deformation of the polyurea.

When $C_{10}$ is adjusted to increase the stiffness of the polyurea, the resulting peak transient deformation is slightly less than that of the material for which $C_{01}$ has been adjusted to achieve the same stiffness. In Figure 5.32, adjusting $C_{10}$ to increase the stiffness gives a greater material toughness (area under the curve) compared to when $\mathrm{C}_{01}$ is adjusted. Thus an increase in $C_{10}$ would be expected to result in increased energy absorption.

When comparing the peak transient deformations of the model for which $C_{01}$ has been increased to double the stiffness, with those attained for the model in which $C_{10}$ has been increased to increase the stiffness by a factor of 1.5, it was found that the former produced lower deformations. Referring to Figure 5.32, the two curves for these material properties have relatively similar stress values for the strain range seen in the numerical models (up to around $120 \%$ ). However, the material model where $C_{01}$ has been increased has a slightly larger area under its curve, thus requiring more energy to deform, resulting in lower deformations. 
Figure 5.34 shows that the de-bonded radius increases as stiffness is increased. The exact reasons for the increase are unclear, however it is likely due to a combination of the way the initial stress wave is transferred through the bond to the polyurea, the rate at which de-bonding occurs, and the geometry of the polyurea/bond/plate interfaces as the de-bonding occurs. Another observation is that the larger de-bonded radii in Figure 5.34 correspond to lower deformations in Figure 5.33. Although a larger de-bonded area will allow a larger area of the polyurea to deform, the corresponding increase in stiffness has a greater effect on the deformation.

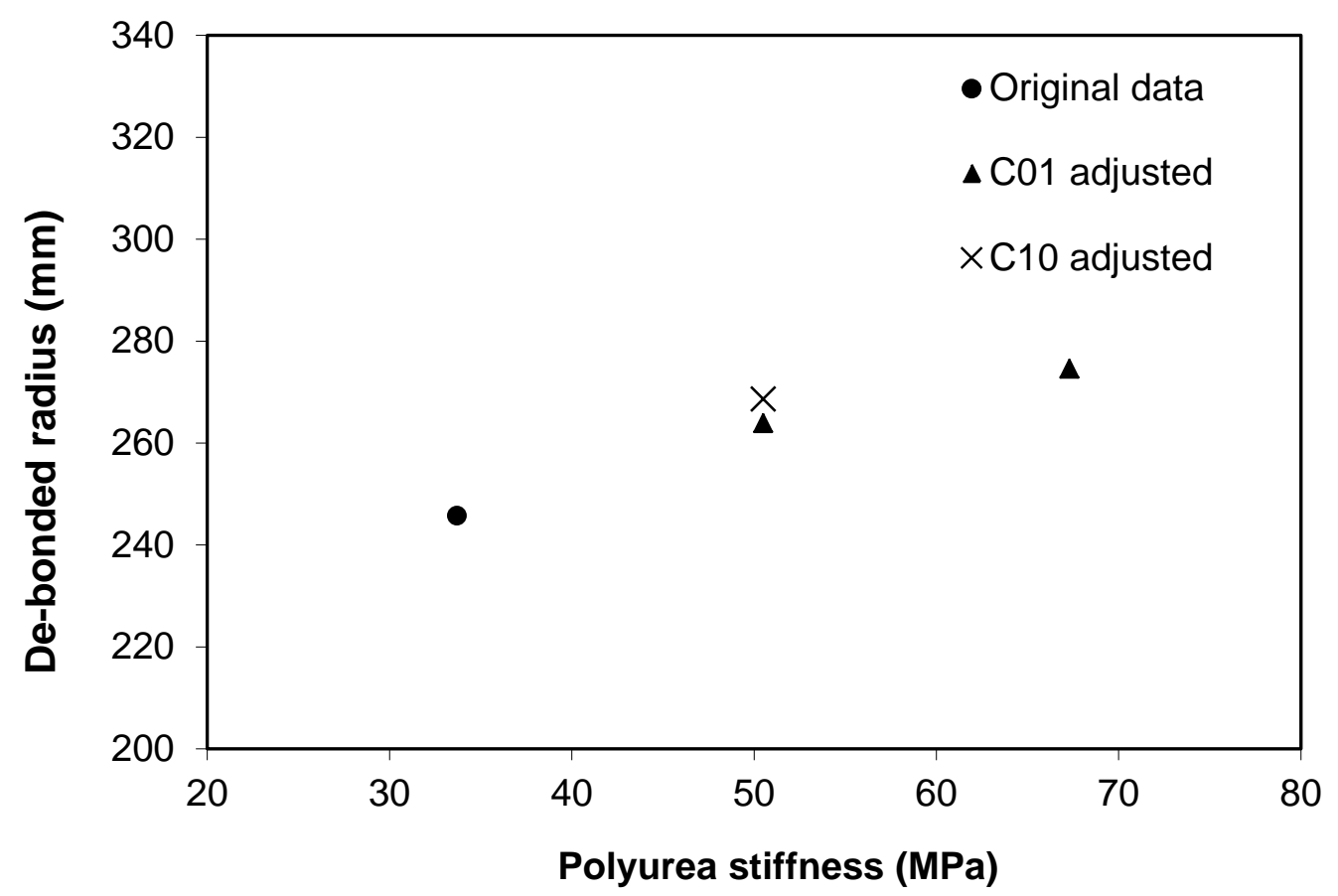

Figure 5.34 Effect of polyurea stiffness on the radius of the de-bonded area.

\subsubsection{Variations in coating bulk modulus}

In this parametric study, the bulk modulus was varied to investigate the effect of using different values reported in literature. The previously validated models used a value of $\kappa=4.98 \mathrm{GPa}$. However values as low as $2.1 \mathrm{GPa}$ (Chakkarapani et al., 2006) have been reported, although the stoichiometry of the polyurea is not reported in this case. Roland and Casalini (2007) report an intermediate bulk modulus of $2.57 \mathrm{GPa}$, however this is for a $95 \%$ stoichiometry polyurea. It is possible that the bulk modulus 
for the polyurea used here could fall anywhere within the range of $2.1 \mathrm{GPa}$ to 4.98 GPa. To investigate the effect of using different values for bulk modulus, the minimum reported value of $2.1 \mathrm{GPa}$ was modelled, as well as an intermediate value of 3.57 GPa (the average of the upper and lower reported values). This was achieved by changing the Mooney-Rivlin constant $d$ to $9.5 \times 10^{-7}$ and $5.6 \times 10^{-7}$ to simulate bulk moduli of $2.1 \mathrm{GPa}$ and $3.57 \mathrm{GPa}$, respectively.

Figure 5.35 shows how the polyurea bulk modulus affects the peak transient deformation of the polyurea. The bulk modulus relates to the material's ability to resist volume changes: the lower the bulk modulus, the more compressible the material. As the bulk modulus is reduced, the peak transient deformation is increased. The more compressible material model allows larger deformations as it can undergo volumetric changes as it elongates. A $34.1 \mathrm{~mm}$ (15\%) difference in peak transient deformation was found when comparing the extremes of the values reported in literature. The differing values may either be due to different polyurea stoichiometries (which were not reported in all the sources) or errors differences in the test procedures. Figure 5.36 shows that the radius of the de-bonded area remains fairly constant as the bulk modulus is varied.

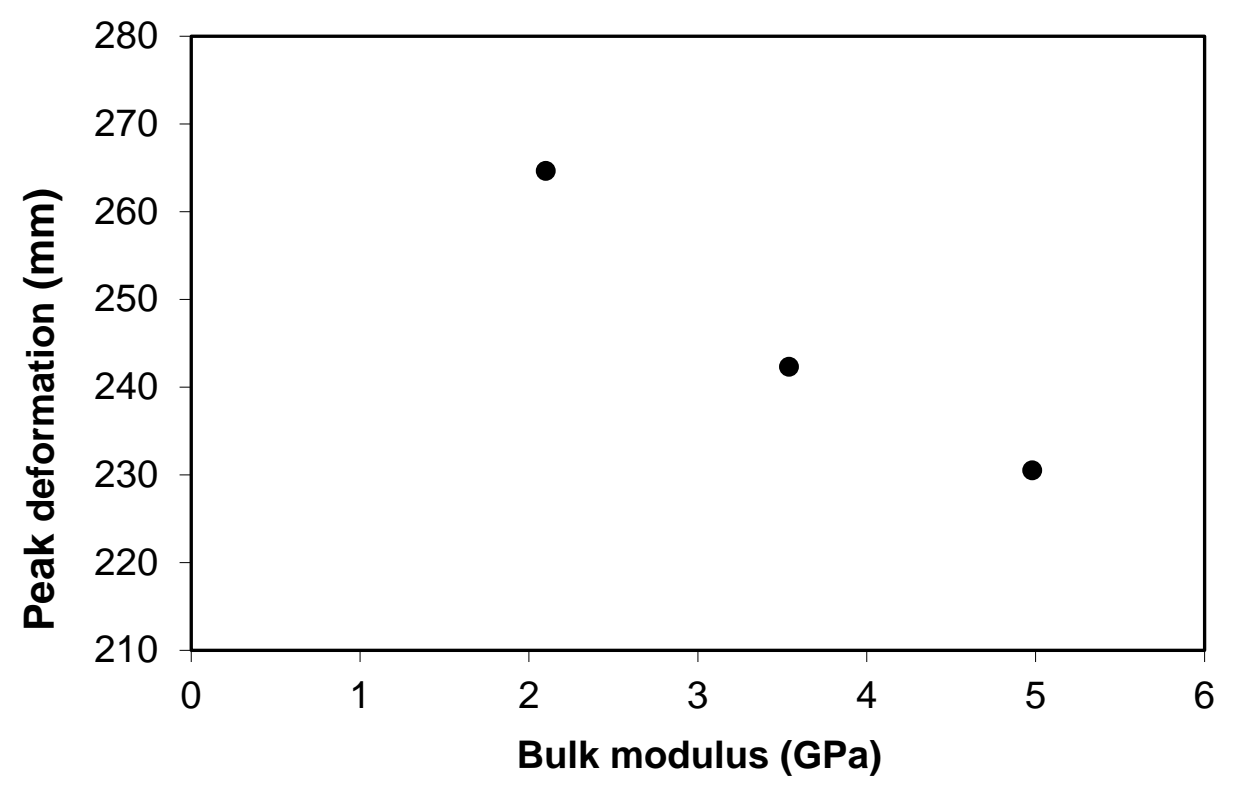

Figure 5.35 Effect of polyurea bulk modulus on peak transient deformation. 


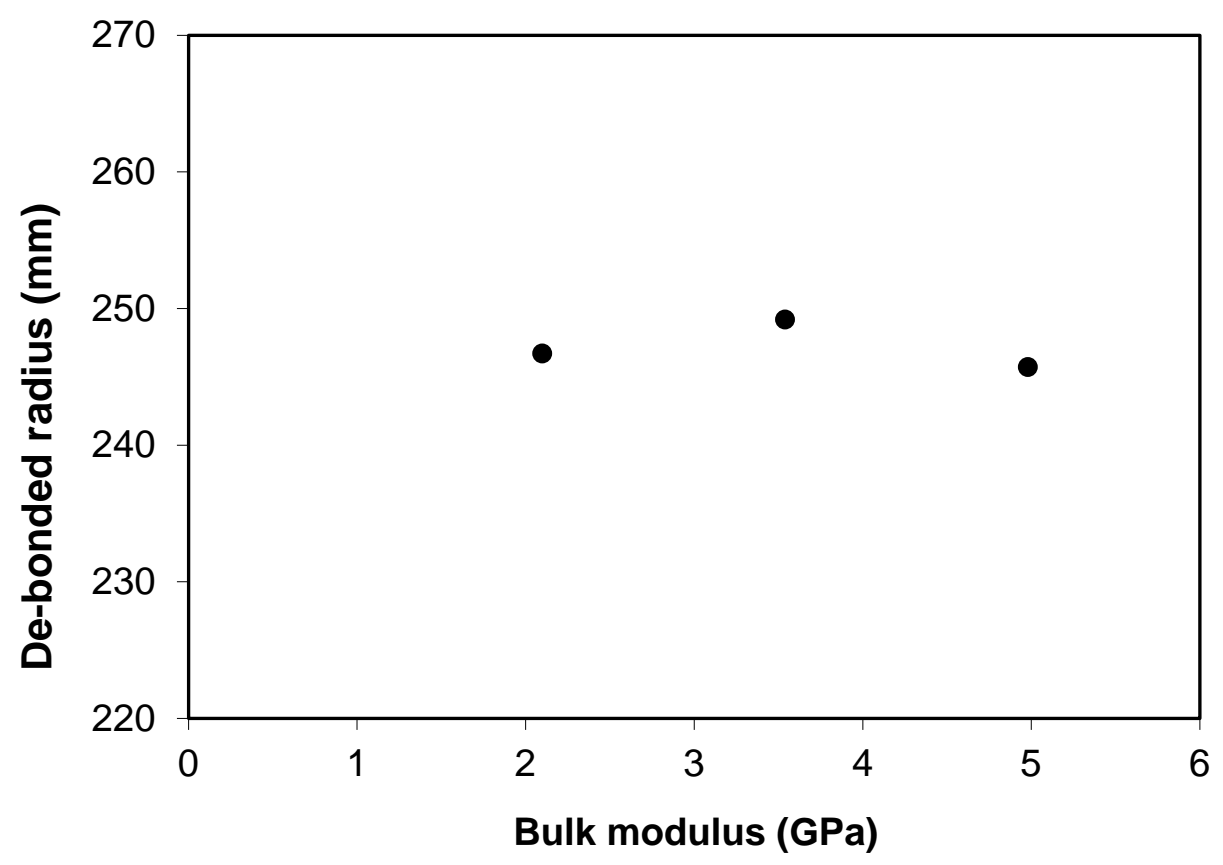

Figure 5.36 Effect of polyurea bulk modulus on the radius of the de-bonded area.

As a result of this study, it can be concluded that the compressibility of polyurea has a significant effect on its deformation under blast loading (Figure 5.35), and under blast loading, a higher bulk modulus is preferable.

\subsubsection{Variations in coating location}

Polyurea coatings were only applied to the back face of the steel plates for the present study. One of the problems found with such applications was that the coatings debonded during the blast events, allowing them to hyper-extend well beyond the peak deformations of the bare steel plates. The application of front face coatings would eliminate this problem; hence this option was investigated numerically as an alternative to back face coatings.

Recent research into the effect of coating location by Amini et al. (2010a, 2010b) found that when polyurea coatings were applied to the back face of impulsively loaded steel plates, part of the loading was dissipated by the polyurea layer. On the other hand, they found that when polyurea coatings are applied to the impact side, the energy that transferred to the plate was increased, and the presence of the coating may 
even promote failure of the plate. Barsoum and Dudt (2010) report experiments in which air-backed plates were subjected to underwater blast, where the presence of polyurea coatings reduced the damage to the panels. They also found that it did not matter significantly whether the polyurea coating was on the front or back of the plate. Thus it appears that the effect of coating location may be dependent on the loading conditions and surrounding medium. This section considers the effect of coating location on the response to localised air-blast.

One of the issues faced with designing the geometric setup when the coatings are applied to the front face during very close-in blast loading is that it is difficult to define the charge stand-off. One option is to keep the stand-off to the back face of the steel plate constant, which is representative of applying a coating to an existing structural component. This is shown as the top diagram in Figure 5.37. However, when the stand-off to the backing material is kept constant, the gap between the charge and the coating is markedly reduced, increasing the loading on the surface of the coating, which becomes an unfair comparison at close range. An alternative method is to maintain a consistent gap between the charge and target surface, as shown in the lower diagram in Figure 5.37. The advantage of this method is that the front face of each plate configuration experiences the same loading condition. However, the concern with method is that it would allow a very thick layer of an extremely lightweight material to be placed on the front surface, hence increasing the stand-off to the steel plate while adding virtually no mass, again leading to an unfair comparison. In the current configuration, due to the proximity of the charge to the plate, it is believed that the second option will provide a more realistic comparison, thus this was used in the simulations. 
Chapter 5. Numerical Simulations and Parametric Studies.

Pentolite

Steel

Option 1: Stand-off to back face of steel plate kept constant

Polyurea

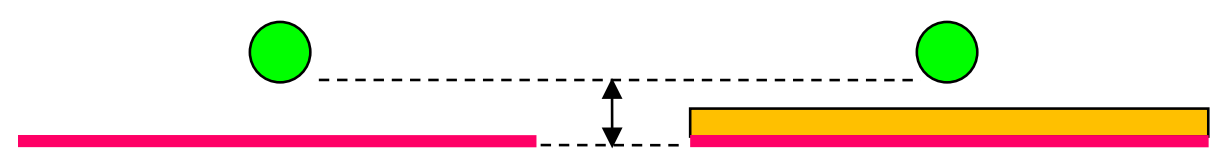

Option 2: Gap between charge and front face kept constant

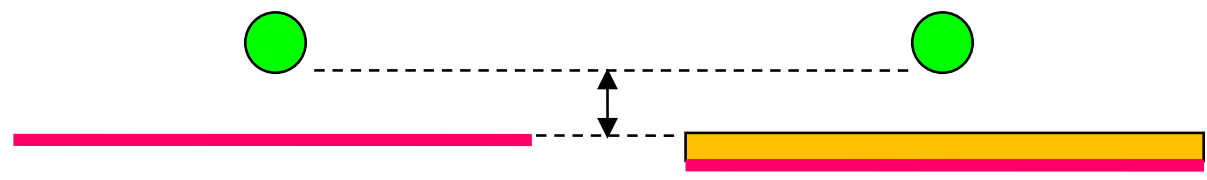

Figure 5.37 Two methods of defining the stand-off for front face coatings.

It should be noted that the polyurea material model parameters used here were derived from tensile data rather than compression data. As the coating on the front face is loaded, the polyurea is loaded in compression. Sarva et al. (2007) showed the nature of the stress-strain curves for polyurea in compression and tension and the stress levels attained for a given strain rate and strain level are similar for strains less than 1.0. Thus it is a reasonable assumption that the model used here will be suitable for this loading scenario as lower strains are expected for the front face coating compared to the back face coating. However, as the model has not been validated for a front coating scenario, these results are an indication only and experimental testing would be required to confirm these findings.

Figure 5.38 shows a sequence of images taken from the numerical model which show the blast response of the polyurea-coated plate with the coating on the front face. The motion of the polyurea away from the blast was restricted by the plate, thus restricting its transient deformation. After being pushed against the plate by the blast load, the elastic response of the polyurea meant that it rebounded off the plate and back towards where the charge had detonated. 
Chapter 5. Numerical Simulations and Parametric Studies.
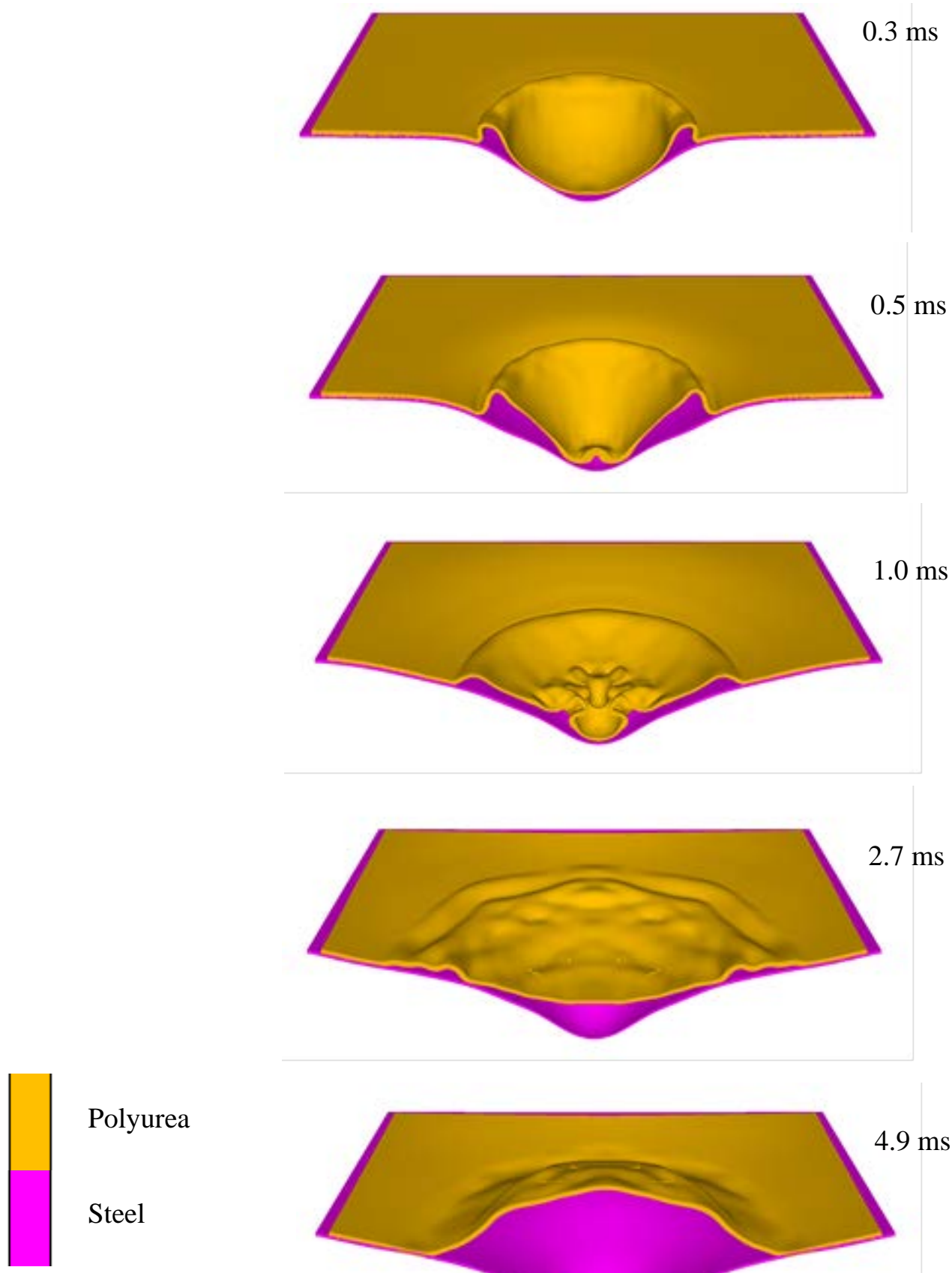

Polyurea

Steel

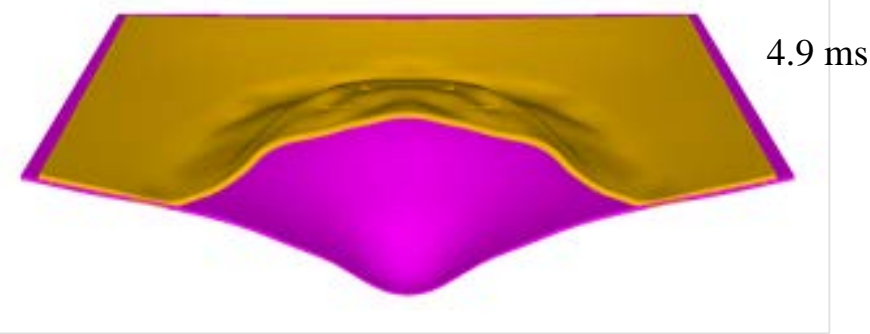

Figure 5.38 Simulation of the blast response of a steel plate with a polyurea coating on the front face. 
The peak transient deformation of the plate was found to be $145.5 \mathrm{~mm}$, whereas when the coating was on the back face, the peak transient deformation of the polyurea was $230.5 \mathrm{~mm}$. Thus, in air-blast situations where polyurea de-bonding is likely to occur, a front face coating is recommended. When compared to a bare steel plate of equivalent areal density, neither coating configuration performs better than the bare steel plate (which had an experimental peak transient deformation of $127 \mathrm{~mm}$ ).

The front-face coating was also compared with a model of a plate with a perfectly bonded polyurea coating on the back face (i.e. with no bond failure model). It was found that the peak transient deformation was 142.5 for the plate with the coating on the back face (no de-bonding), compared to $145.5 \mathrm{~mm}$ for the plate with the coating on the front face (with de-bonding). This $2 \%$ difference suggests that the de-bonding of the polyurea on the front face has little effect on the peak transient deformation, and that the reduction compared to the back-face coating is primarily due to the restriction in motion caused by the steel plate.

In addition to a front face coating, a configuration in which the polyurea was sandwiched between two steel plates was considered. This was investigated by simulating a polyurea with a thickness of $7.7 \mathrm{~mm}$, sandwiched between two steel plates of $2.5 \mathrm{~mm}$ thickness. The stand-off between the charge and the front surface of the front plate was set to $10 \mathrm{~mm}$, to be consistent with previous models.

Figure 5.39 shows a sequence of images from the steel/polyurea/steel sandwich plate simulation. De-bonding was found to occur at both interfaces between the steel plates and the polyurea. The peak transient deformation of this solution was found to be $150.4 \mathrm{~mm}$, which is higher than the result for the front face coated plate. The reduced deformation resistance of this plate configuration is believed to be due to the reduced structural stiffness when the plate components have de-bonded. Despite deforming more than the front face coated plate, this solution had a lower peak transient deformation than the back face coated plate, as the hyperextension of the de-bonded polyurea is contained. 

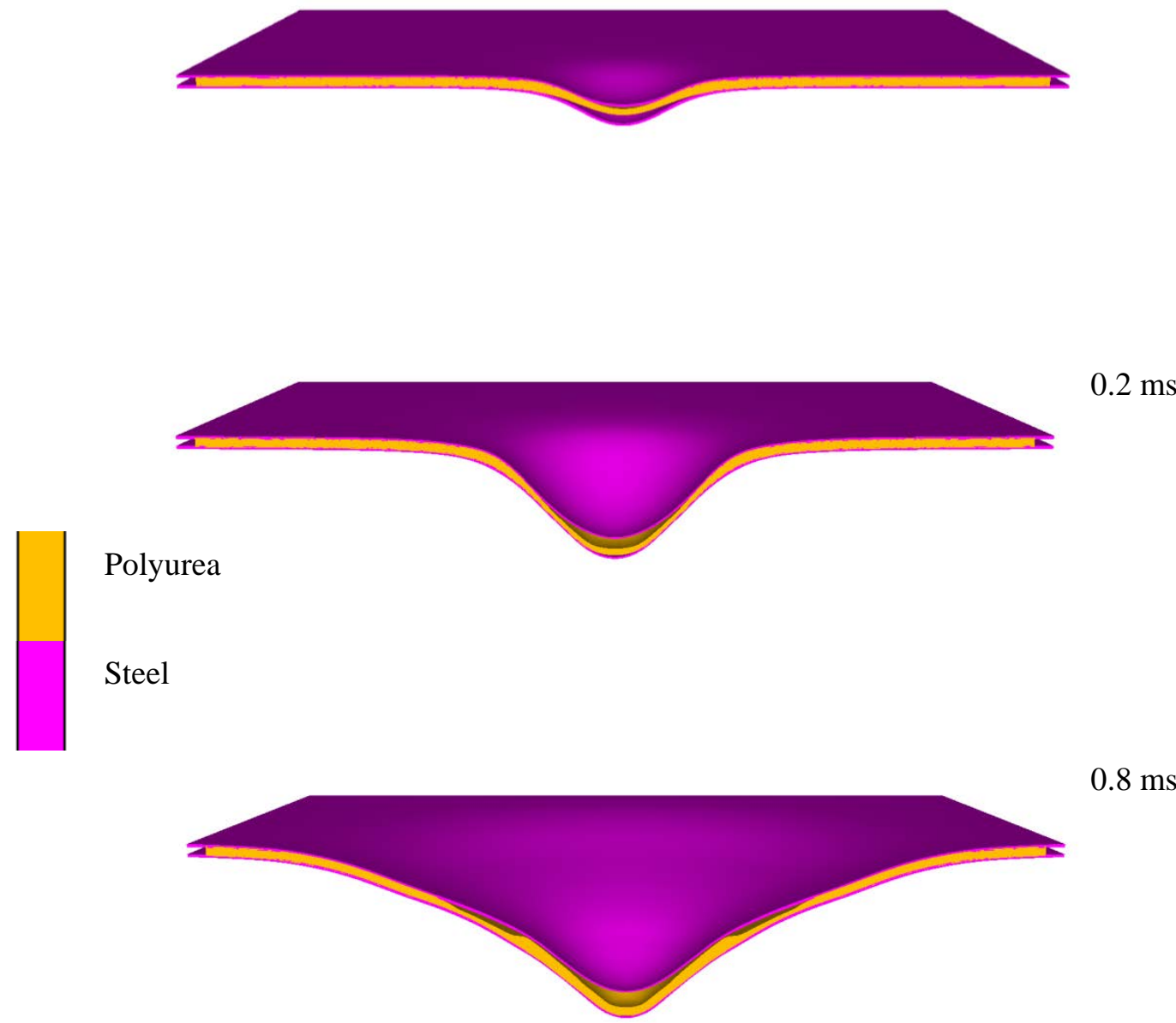

Figure 5.39 Simulation of the blast response of a plate with a steel/polyurea/steel sandwich configuration.

The coating location has been found to play an important role in the blast deformation resistance of polyurea-coated steel plates. In the experimental work (Chapter 4), it was found that the coatings can de-bond under blast loading. By applying the coating to the front face (blast side) of the plate instead of the back, the motion of the polyurea is restricted by the plate, thus reducing the transient deformation. 


\subsection{Development of a strain-rate dependent Mooney-Rivlin model for polyurea}

The 2-parameter Mooney-Rivlin model used in the previous sections provided close agreement between the numerical model and experimental results, and was sufficient to conduct the parametric studies. However, characterisation tests showed a clear dependency of the material stress-strain behaviour on strain rate (see Figure 5.21). Although it is expected that Mooney-Rivlin parameter fits to the characterisation data at 10 /s will provide a reasonable reproduction of the material deviatoric behaviour, once the polyurea de-bonds from the steel plate it rapidly deforms at a higher rate. Thus, a strain-rate independent model is incapable of accurately capturing the full range of polyurea behaviour during the blast event. By including strain-rate sensitivity, the material model will also be applicable over a wider range of problems. In this section, a strain-rate dependent 5-parameter Mooney-Rivlin material model is fit to the polyurea tensile test data.

A 5-parameter Mooney-Rivlin model was selected in order to capture the inflection in the quasi-static tensile test data (see Figure 5.21). The 5-parameter Mooney-Rivlin model is derived from Equation 2.8 and given by (Mac Donald, 2007):

$$
\begin{aligned}
\psi= & C_{10}\left(I_{1}-3\right)+C_{01}\left(I_{2}-3\right)+C_{20}\left(I_{1}-3\right)^{2}+C_{11}\left(I_{1}-3\right)\left(I_{2}-3\right)+ \\
& C_{02}\left(I_{2}-3\right)^{2}+\frac{1}{d}(J-1)^{2}
\end{aligned}
$$

Using equations 2.10, 2.11 and 5.1, the engineering stress equation for the 5-parameter Mooney-Rivlin model can be found to be:

$$
\begin{aligned}
\sigma= & 2\left(\lambda-\lambda^{-2}\right)\left[\left(C_{10}+2 C_{20}\left(\lambda^{2}+2 \lambda^{-1}\right)-6 C_{20}+C_{11}\left(2 \lambda+\lambda^{-2}\right)-\right.\right. \\
& \left.\left.3 C_{11}\right)+\lambda^{-1}\left(C_{01}+C_{11}\left(\lambda^{2}+2 \lambda^{-1}\right)-3 C_{11}+2 C_{02}\left(2 \lambda+\lambda^{-2}\right)-6 C_{02}\right)\right]
\end{aligned}
$$

Equation 5.2 was used to develop a strain-rate dependent material model for polyurea, based on the tensile test data shown in Figure 5.21. The strain-rate dependency was achieved by varying the constants $C_{10}, C_{01}, C_{11}, C_{20}$ and $C_{02}$. The data analysis software Origin ${ }^{\circledR}$ was first used to apply a least-squares regression method to fit a curve in the form of Equation 5.2 to each of the curves in Figure 5.21. The resulting 
constants were then plotted against strain rate, and linear relationships were fit to a filtered set of data points (quasi-static and $100 / \mathrm{s}$ ) for constants $C_{10}, C_{01}$ and $C_{20}$. This process was used to simplify the rate-dependency expressions of the initial equation constants, and to ensure consistency in the final fit curves. Using the linear, rate dependent expressions for $C_{10}, C_{01}$ and $C_{20}$, Equation 5.2 was fit to the stress-strain data in Figure 5.21 to determine values for $C_{11}$ and $C_{02}$. Constants $C_{11}$ and $C_{02}$ were plotted against strain rate and a power relationship was fit to the data. The resulting equations for the constants in Equation 5.2 in terms of the strain rate were:

$$
\begin{aligned}
& C_{10}=-21.16-0.14652 \times \dot{\varepsilon} \\
& C_{01}=32.04+0.178 \times \dot{\varepsilon} \\
& C_{11}=-43.18572+41.96492 \times 0.999415^{\dot{\varepsilon}} \\
& C_{20}=0.185+0.0037 \times \dot{\varepsilon} \\
& C_{02}=119.81158-111.9635 \times 0.99923^{\dot{\varepsilon}}
\end{aligned}
$$

The resulting curve fit is compared with the experimental data in Figure 5.40. Its validity is restricted to stretch ratios up to 4 (strains up to 3) and strain rates up to $200 \mathrm{~s}^{-1}$. The fitted curves match the experimental data well, with the largest discrepancies seen for the quasi-static data and the $20 \mathrm{~s}^{-1}$ curve. The quasi-static curve fit over-predicts the stress by up to $11 \%$ above stretch ratios of 2 , and the $20 \mathrm{~s}^{-1}$ curve fit under-predicts the stress by up to $10 \%$ above stretch ratios of 1.5 . Despite this, the curve fit is a significant improvement compared to the previous 2-parameter strainrate independent fit in terms of capturing the polyurea behaviour at a wide range of strain rates. 


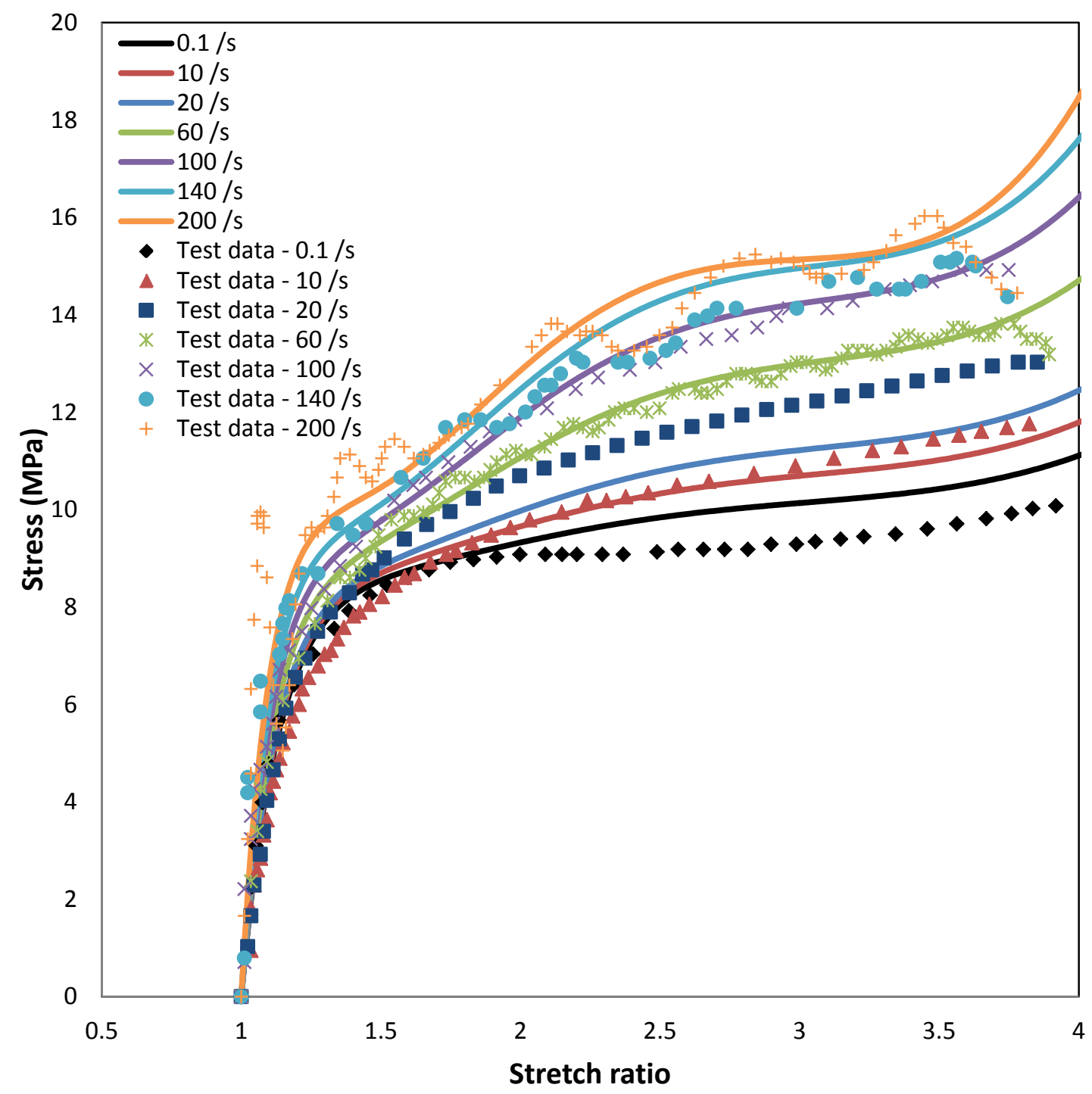

Figure 5.40 Strain-rate dependent 5-parameter Mooney-Rivlin curve fits to experimental tensile test data of polyurea.

Currently, the user subroutines in AUTODYN do not enable the customisation of hyperelastic material models (such as Mooney-Rivlin) (Quan, C, ANSYS Lead Technical Services Engineer, 2013, pers. comm., 24 Jan). As such, the strain-rate dependency extension of the five-parameter Mooney-Rivlin model cannot, at this stage, be implemented. However, a user-defined subroutine for hyperelastic materials will be made available in the next release of AUTODYN (Version 15.0), which will allow this model to be implemented. The extension proposed may also be suitable for implementation in other hydrocodes (e.g. LS-DYNA, CTH, etc.), and discussions are 
currently taking place with ANSYS developers to add this functionality to AUTODYN.

\subsection{Summary}

In this chapter, the numerical models from Chapter 3 were modified to simulate the explosive blast testing presented in Chapter 4. In the blast tests, it was observed through high speed imagery that the polyurea coatings de-bonded from the plate, resulting in large transient deformations. This de-bonding was incorporated into the models by using a thin layer of elements to represent the bond, which failed at a principal stress value of $80 \mathrm{MPa}$. This simple empirical model was effective in producing polyurea de-bonding in the simulation; however it may not be an accurate physical representation.

The numerical models run with the Mooney-Rivlin material constants introduced in Chapter 3 agreed closely with the experimental results for residual deformation, but did not accurately capture the transient deformation of the polyurea. In order to produce a better representation of the material properties, high strain rate tensile testing was conducted on the polyurea. New material model constants were fitted to the data at a strain rate of $10 \mathrm{~s}^{-1}$, which is typical of the strain rates seen in the numerical models. The new Mooney-Rivlin constants gave an improved correlation with the experimental results compared to the previous model, with peak transient deformations within $10 \%$ of the experimental results.

Once validated, the numerical model was used to conduct parametric studies to investigate the effect of bond strength, polyurea stiffness, polyurea bulk modulus and the coating location on blast performance. It was found that the greatest improvement in terms of transient deformation resistance could be achieved by changing the coating location to the front (blast side) face instead of the back face. This meant that the motion of the polyurea was restricted by the plate; hence much lower transient deformations were seen. The coating performance could also be improved by increasing the polyurea stiffness or bulk modulus, or increasing the strength of the bond between the polyurea and the plate. None of the coated plates in the parametric 
studies performed as well as a mass-equivalent steel plate. However, coated plates may still be preferable for other reasons. For example, when an appliqué (add-on) armour is required, polyurea may be chosen as it is more easily applied than metallic armour and the added mass of the polymer may improve the performance compared to the original (uncoated) armour.

Finally, a strain rate dependent 5-parameter Mooney-Rivlin model was presented. The model was derived from tensile test data with strains up to $300 \%$ and strain rates up to $200 \mathrm{~s}^{-1}$. Beyond these conditions it should not be applied. Although AUTODYN does not currently have the functionality to implement such a model, it may be implemented into future releases for improved accuracy of variable strain-rate problems. 


\section{Chapter 6}

\section{Summary and Recommendations}

This chapter summarises the main findings and outcomes presented in this thesis, followed by recommendations for further work based on the findings.

\subsection{Chapter 2: Literature review and identification of knowledge gaps}

In the early stages of the research, a literature review was conducted to identify knowledge gaps in the area of polyurea coatings for blast protection. The review was continually updated throughout the duration of the project to monitor any new additions to the knowledge in the area. One of the gaps identified was that it was unclear whether a polyurea-coated steel plate or a mass-equivalent steel plate will perform better under blast loading. It was also unclear what parameters (e.g. material properties, coating location, etc.) would most affect the performance. It was proposed that experimental testing and experimentally-validated numerical models were used to investigate such topics.

\subsection{Chapter 3: Preliminary experiments and numerical validation}

Preliminary experimental and numerical studies were carried out to develop and validate numerical models of polyurea-coated plates under blast loading. Explosive blast tests using bare steel plates were conducted to provide validation data together with results from Ackland et al. (2007) on polyurea-coated steel. The experimental data included residual deformation measurements from 3D laser scans, as well as transient deformation data (for the bare steel plates only).

The hydrocode ANSYS ${ }^{\circledR}$ AUTODYN ${ }^{\circledR}$ was used to conduct numerical modelling of the validation experiments. Initially, a comprehensive investigation into the mesh sizes required to accurately model the blast load and plate deformation was 
conducted. Once appropriate mesh sizes were determined, an Euler-Lagrange coupled analysis was used to simulate the plate response to the localised air-blast loading and compared with experimental results. Both 4-noded shell and 8-noded hex elements were trialled for use in modelling. When compared with the experimental results, the model using hex elements under-predicted the plate deformation by around a factor of two. This was believed to be due to shear locking, and as a result shell elements were selected for subsequent models. The models using shell elements gave good agreements for the residual plate deformations (within 2\% of the experimental results for the bare plates and within $10 \%$ for the polyurea-coated plates), however they slightly under-predicted the springback and peak transient deformations of the bare plates. The successful application of the shell elements also demonstrated that the deformation could be faithfully accounted for by membrane and bending forces alone (i.e. capturing the through thickness stress wave was not required for accurate modelling).

\subsection{Chapter 4: Explosive testing of polyurea-coated steel plates}

Chapter 4 described a series of explosive blast tests which was designed to determine whether the application of polyurea coatings to steel plates can reduce their deformation under blast loading, compared to uncoated steel plates with an equivalent areal density. The experiments also investigated how the ratio of polyurea to steel affects the deformation in mass-equivalent plates.

A new experimental blast rig was designed for the experimental series. The rig was designed to incorporate a number of types of instrumentation, including a high speed camera and a laser displacement transducer.

The average peak residual deformations of the plates were found to increase with coating thickness. Increases of $8 \%$ and $18 \%$ were found for the $5 \mathrm{~mm}$ steel plates with $7.7 \mathrm{~mm}$ polyurea coatings and $4 \mathrm{~mm}$ steel plates with $15.7 \mathrm{~mm}$ polyurea coatings, respectively, when compared to the $6 \mathrm{~mm}$ uncoated steel plates. This showed that that although polyurea coatings can be applied as an appliqué to steel plates in order to reduce their residual deformation (Ackland et al., 2007), bare steel plates are more 
effective in reducing residual plate deformation when compared with polyurea-coated plates of equivalent mass.

The transient deformation of the plate/polyurea is a more useful measure of performance than residual deformation as it defines the distance behind the plate within which damage can occur. A high speed camera was used to capture the transient deformation at the plate centre, and the footage revealed that the polyurea coatings de-bonded during the events. As a result, the plates with polyurea coatings on the back face had approximately double the peak transient deformation compared to the bare steel plates. Methods to reduce the peak transient deformation were investigated as part of the parametric studies in Chapter 5.

\subsection{Chapter 5: Numerical simulation and parametric studies}

The preliminary numerical models developed and validated in Chapter 3 were modified to match the geometry and setup of the experiments described in Chapter 4. The models were also enhanced to simulate de-bonding of the polyurea coatings by adding a thin layer of elements between the polyurea and the plate, which failed at a nominated stress value. It was found that the bond strength between the polyurea and the plate which best matched the experiments was $80 \mathrm{MPa}$. It should be noted that the bond model used in this thesis was a simplified empirical model that requires further development to accurately model the physical behaviour of the de-bonding process.

The models were validated against the explosive test results of polyurea-coated steel plates, and found to correlate very well for residual deformation. However, the transient deformations of the polyurea coatings (after de-bonding) were underpredicted. To more accurately simulate the polyurea behaviour at high strains after debonding, the Mooney-Rivlin material model constants were also modified. Uniaxial tensile testing was conducted at a variety of strain rates to provide data suitable for capturing the polyurea behaviour under very close-in (detonics regime) blast loads. Representative strain rates of $10 \mathrm{~s}^{-1}$ and strains of $100 \%$ were extracted from the numerical simulations and used to derive the new Mooney-Rivlin constants. The new

constants gave improved correlation with experimental results, and can easily be entered into commercially available hydrocodes and finite element codes. 
Parametric studies were then performed to investigate the effects of the bond strength between the polyurea coating and the plate, the polyurea stiffness, the polyurea bulk modulus, and the location of the polyurea coating with respect to the plate to be studied. It was found that increasing the bond strength between the polyurea coating and the plate improved its performance, albeit minimally. A doubling of the bond strength from 80 to $160 \mathrm{MPa}$ decreased the transient deformation by $4.6 \%$.

It was found that increasing the polyurea stiffness resulted in lower transient deformations of the polyurea. However, changing the stiffness also had an effect on the area under the stress-strain curve, which is related to the ability of the material to absorb energy as it deforms. For materials with the same stiffness, the material combinations with lower energy absorptions resulted in lower deformations. The disadvantage to increased polyurea stiffness is that it also results in a slightly larger de-bonded area. However, this is only a problem for components whose size is similar to, or less than, the expected de-bonded area.

The bulk modulus of polyurea was found to significantly affect deformation under blast loading. It was found that the values for bulk modulus reported in literature varied widely, introducing a large uncertainty in the numerical simulation of such materials. A range of bulk moduli from the literature were simulated, and the results showed that the higher the bulk modulus, the lower the deformation of the polyurea. Changing the bulk modulus from $2.1 \mathrm{GPa}$ to $4.98 \mathrm{GPa}$ resulted in a $13 \%$ reduction in peak transient deformation. Also, the bulk modulus was found to have little effect on the de-bonded area. Thus a higher bulk modulus is preferred for this blast loading scenario.

It was found that the most effective way to improve the performance of a polyurea-coated plate was to apply the coating to the front face of the steel plate instead of the back face. Although the polyurea still de-bonds, its motion is restricted by the plate, thus reducing peak transient deformation and hence the potential damage zone behind the plate. Although a front face coating is preferable to a coating on the back face, a steel plate of equivalent mass outperforms either configuration. However, this may not be an option for vehicle or structural upgrades (i.e. when appliqué armour is required). A sandwich plate configuration was also investigated, which was 
less effective in reducing transient deformation than the front face coating. However, it was more effective than the back face coating, due to the plate opposite the blast side restricting the motion of the polyurea.

A strain rate dependent 5-parameter Mooney-Rivlin model was presented to provide improved accuracy over a range of strain rates. Although AUTODYN does not currently have the functionality to implement the model, it may be implemented into future releases for improved accuracy of variable strain-rate problems.

\subsection{Outcomes}

As a result of this research, a sound method for experimentally testing and numerically modelling the deformation of polyurea-coated plates under blast loading has been developed. This includes high strain rate tensile test results and MooneyRivlin material model constants for polyurea which may be used as an input in finite element codes to further investigate polyurea coatings for blast and high strain rate loading scenarios.

Although polyurea-coated steel plates were found not to perform as well as bare steel plates in terms of reducing peak transient deformations under blast (for the same areal density), they may be applied in protective systems for other reasons. Hence it is important to have the ability to more reliably predict the blast response of such protective systems.

Parametric studies allowed the effects of bond strength between the polyurea coating and the plate, the polyurea stiffness, the polyurea bulk modulus, and the location of the polyurea with respect to the plate to be studied. The outcomes of these studies show that each of these parameters has an effect on the performance of the polyureacoated plate under blast loading. The results potentially allow polyurea coatings to be modified in order to improve their performance. Such improvements may be made if the polyurea stiffness, bulk modulus or polyurea-metal bond strength can be increased, or if the coating is applied to the front face of the steel. 


\subsection{Recommendations for further work}

As a result of this research, a number of areas for future work have been identified and are presented in the next section.

\subsubsection{Implementation of the strain-rate dependent 5-parameter Mooney- Rivlin model into hydrocodes}

In Section 5.6, a 5-parameter strain-rate dependent Mooney-Rivlin model was derived for polyurea. The inclusion of strain-rate effects should result in a more accurate model, applicable to a wider range of problems than compared to the baseline 2-parameter Mooney-Rivlin model. Currently, AUTODYN does not allow the implementation of such a model. However, a user-defined subroutine for hyperelastic materials will be made available in the next release of AUTODYN (Version 15.0), which will allow this model to be implemented. The fits presented may also be suitable for implementation into another hydrocode (e.g. LS-DYNA). In addition, further mechanical testing (for example confined and unconfined compression tests) would allow additional improvements the model by improving the accuracy of the bulk modulus property.

\subsubsection{Further investigation into bond strength}

In the parametric studies presented in this thesis, a simplified principal stress failure model was used to model the bond between the plate and the polyurea coating. Although sufficient for the studies presented here, the bond strength and failure modes could be further investigated to develop more accurate models of the debonding of the polyurea. This may include simulation of the stress wave propagation within the steel plate and an investigation into how it transfers through the bond to the polyurea. The bonding parameters of the interface between the polyurea and the steel plate could be evaluated experimentally, for example through specially designed pullout tests, at various strain rates. The results could then be used to improve the numerical models of polyurea de-bonding. 


\subsubsection{Polyurea to prevent plate rupture}

The work in this thesis has shown that polyurea-coated plates do not reduce deformation under blast loading compared to bare steel plates of equivalent areal density. However, recent studies suggest that such coatings may be able to prevent plate rupture (Amini et al., 2010a, Amini et al., 2010b, Barsoum and Dudt, 2010). Further research into how polyurea prevents plate rupture could be investigated by extending the experimental and numerical methods developed in this thesis. This could also be expanded to investigate whether polyurea coatings can prevent weld ruptures (or at least increase the strength of welded joints) under blast loading.

\subsubsection{Polyurea to protect against ballistic penetration}

Although blast is one potential threat to structures, other threats may be considered when designing a protection system. Xue et al. (2010) found that steel plates backed with polyurea provide additional resistance to penetrators compared with the same plates without coatings. El Sayed et al. (2009) successfully simulated and validated a ballistic impact on a polyurea retrofitted high strength structural steel plate. Future experimental and numerical studies may build on the work presented here to further investigate the ability of polyurea to prevent penetration.

\subsubsection{Polyurea combined with other materials}

Future work may investigate combining polyurea with other materials in either a layered or sandwich plate configuration or as a matrix in a composite material. The use of polyurea in sandwich panels under blast loading has been investigated by a number of authors (Bahei-El-Din and Dvorak, 2007, Bahei-El-Din et al., 2006, Gardner et al., 2012) as well as in composite panels (Hui and Oskay, 2012, Kim et al., 2012, McShane et al., 2008, Tekalur et al., 2008). Experimental and numerical testing using the methods developed in this thesis could be applied to further understand the effect of sandwich plates containing polyurea and how they might be used to provide blast protection. Research has also been conducted into the use of polyurea as the matrix of a composite material. For example, Billaudeau (2010) studied the effect on mechanical properties when nanoparticles are dispersed in a polyurea matrix. Such 
Chapter 6. Summary and Recommendations.

materials could be evaluated to determine whether they can provide improved blast protection compared to the polyurea-coated plates in this thesis. 


\section{References}

Ackland, K., Anderson, C. \& St John, N. (2007). "Polymeric coatings for enhanced protection of structures from the explosive effects of blast". In: Mendis, P., Lai, J., Dawson, E. \& Abbass, H., eds. Recent advances in security technology 2007, Proceedings of the RNSA Security Technology Conference, 28 September 2007, Melbourne, Australia. Australian Homeland Security Research Centre, pp. 90-96.

Amini, M.R. (2007). Effect of polyurea on dynamic response and fracture resistance of steel plates under impulsive loads. Ph.D. thesis, University of California.

Amini, M.R., Amirkhizi, A.V. \& Nemat-Nasser, S. (2010a). "Numerical modeling of response of monolithic and bilayer plates to impulsive loads". International Journal of Impact Engineering, Vol. 37, pp. 90-102.

Amini, M.R., Isaacs, J. \& Nemat-Nasser, S. (2006). "Effect of polyurea on the dynamic response of steel plates". In: 2006 SEM Annual Conference and Exposition on Experimental Applied Mechanics, 4-7 June, 2006, St. Louis, MO.

Amini, M.R., Isaacs, J. \& Nemat-Nasser, S. (2010b). "Experimental investigation of response of monolithic and bilayer plates to impulsive loads". International Journal of Impact Engineering, Vol. 37, pp. 82-89.

Amirkhizi, A.V., Isaacs, J., Mcgee, J. \& Nemat-Nasser, S. (2006). "An experimentally-based viscoelastic constitutive model for polyurea, including pressure and temperature effects". Philosophical Magazine, Vol. 86, pp. 58475866.

Anderson, C.E. (1987). "An overview of the theory of hydrocodes". International Journal of Impact Engineering, Vol. 5, pp. 33-59.

ANSYS (2005). AUTODYN Theory Manual, version 4.3. Century Dynamics Inc: CA, USA.

ANSYS (2008). Release 11.0 Documentation for ANSYS Workbench. Century Dynamics Inc: CA, USA.

ANSYS AUTODYN (2009). Version 12.1. ANSYS Inc: PA. 
ASTM International (2008). E 8/E 8M - 08. Standard test methods for tension testing of metallic materials.

Australian Government Department of Defence. (2012). Australian Operation in Afghanistan [Online]. Available:

http://www.defence.gov.au/op/afghanistan/info/factsheet.htm [Accessed 3 December 2012].

Bahei-El-Din, Y.A. \& Dvorak, G.J. (2007). "Behavior of sandwich plates reinforced with polyurethane/polyurea interlayers under blast loads". Journal of Sandwich Structures and Materials, Vol. 9, pp. 261-281.

Bahei-El-Din, Y.A., Dvorak, G.J. \& Fredricksen, O.J. (2006). "A blast-tolerant sandwich plate design with a polyurea interlayer ". International Journal of Solids and Structures, Vol. 43, pp. 7644-7658.

Baker, W.E. (1975). "Approximate techniques for plastic deformation of structures under impulsive loading". Shock and Vibration Digest, Vol. 7, pp. 107-117.

Baker, W.E., Cox, P.A., Westine, P.S., Kulesz, J.J. \& Strehlow, R.A. (1983). Explosion hazards and evaluation, Amsterdam, Elsevier Scientific Publishers B.V.

Balden, V.H. \& Nurick, G.N. (2005). "Numerical simulation of the post-failure motion of steel plates subjected to blast loading". International Journal of Impact Engineering, Vol. 32, pp. 14-34.

Barsoum, R.G.S. \& Dudt, P.J. (2010). "The fascinating behaviors of ordinary materials under dynamic conditions". AMMTIAC Quarterly Vol. 4, No. 4 Countering the Modern Threat: Armor for Advanced Protection.

Belytschko, T., Lin, J.I. \& Tsay, T.S. (1984). "Explicit algorithms for the nonlineardynamics of shells". Computer Methods in Applied Mechanics and Engineering, Vol. 42, pp. 225-251.

Beveridge, A. (1998). Forensic investigation of explosions, Taylor \& Francis.

Billaudeau, E. (2010). Mechanical behavior of polyurea nanocomposites doped with nanoparticles, Research Report, Ecole Centrale de Lyon.

Bluescope Steel. (2009). Bluescope Steel 350 Xlerplate ${ }^{\circledR}$ steel datasheet [Online]. Available: http://www.xlerplate.com.au/go/product/as/nzs-3678-350-xlerplate [Accessed 24 November 2010]. 
Bogoslovov, R.B., Roland, C.M. \& Gamache, R.M. (2007). "Impact-induced glass transition in elastomeric coatings". Applied Physics Letters, Vol. 90, pp. 19101913.

Çengel, Y.A. \& Boles, M.A. (1994). Thermodynamics: an engineering approach, USA, McGraw-Hill, Inc.

Chakkarapani, V., Ravi-Chandar, K. \& Liechti, K.M. (2006). "Characterizarion of multiaxial constitutive properties of rubbery polymers". Journal of Engineering Materials and Technology, Vol. 128, pp. 489-494.

Charlton, D.J., Yang, J. \& Teh, K.K. (1994). "A review of methods to characterize rubber elastic behavior for use in finite element analysis". Rubber Chemistry and Technology, Vol. 67, pp. 481-503.

Chen, C., Linzell, D.G., Alpman, E. \& Long, L.N. (2008). "Effectiveness of advanced coating systems for mitigating blast effects on steel components". In: 10th International Conference on Structures Under Shock and Loading, Algarve, Portugal.

Chon, C.T. \& Symonds, P.S. (1977). "Large dynamic deflection of plates by mode method". Journal of Engineering Mechanics Div., Proceedings of the ASCE, Vol. 103, pp. 3-14.

Cook, R.D., Malkus, D.S. \& Plesha, M.E. (1989). Concepts and applications of finite element analysis, Canada, John Wiley \& Sons.

Davidson, J.S., Fisher, J.W., Hammons, M.I., Porter, J.R. \& Dinan, R.J. (2005). "Failure mechanisms of polymer-reinforced concrete masonry walls subjected to blast". Journal of Structural Engineering, Vol. 131, pp. 1194-1205.

Davidson, J.S., Porter, J.R., Dinan, R.J., Hammons, M.I. \& Connell, J.D. (2004). "Explosive testing of polymer retrofit masonry walls". Journal of Performance of Constructed Facilities, Vol. 18, pp. 100-106.

Davidson, J.S. \& Sudame, S. (2004). Development of computational models and input sensitivity study of polymer reinforced concrete masonry walls subjected to blast, AFRL-ML-TY-TR-2006-4522, Florida, Air Force Research Laboratory.

Drotleff, J.E., Vincent, T.C., Mullin, S.A., Walker, J.D. \& Morris, B.L. (1996). Research in close-in blast loading from high explosives, ARL-CR-308, CA, Army Research Laboratory. 
Duffey, T.A. (1967). The large deflection dynamic response of clamped circular plates subject to explosive loading, Sandia Laboratories Research Report No. SC-RR-67-532, Sandia Laboratories.

El Sayed, T., Mock, W., Mota, A., Fraternali, F. \& Ortiz, M. (2009). "Computational assessment of ballistic impact on a high strength structural steel/polyurea composite plate". Computational Mechanics, Vol. 43, pp. 525-534.

Finney, R.H. \& Kumar, A. (1987). "Development of material constants for nonlinear finite-element analysis". In: Presented at a meeting of Rubber Division, American Chemical Society, 6-9 October, Cleveland, Ohio.

Friedlander, F.G. (1946). "The Diffraction of Sound Pulses. I. Diffraction by a SemiInfinite Plane". Proceedings of the Royal Society of London. Series A. Mathematical and Physical Sciences, Vol. 186, pp. 322-344.

Gardner, N., Wang, E., Kumar, P. \& Shulka, A. (2012). "Blast mitigation in a sandwich composite using graded core and polyurea interlayer". Experimental Mechanics, Vol. 52, pp. 119-134.

Gayle, J.B. \& Bransford, J.W. (1965). Size and duration of fireballs from propellant explosives, NASA TM X-53314.

Gere, J.M. \& Timoshenko, S.P. (1997). Mechanics of Materials, Boston, PWS Publishing company.

Grujicic, M., Pandurangan, B., Zecevic, U., Koudela, K. \& Cheeseman, B. (2007). "Ballistic performance of alumina/S-2 glass-reinforced polymer-matrix composite hybrid lightweight armor against armor piercing (AP) and non-AP projectiles". Multidiscipline Modeling in Materials and Structures, Vol. 3, pp. 287-392.

Hammond, L. (2000). The structural response of submerged air-backed plates to underwater explosions. Ph.D. Thesis, Monash University.

Hiermaier, S.J. (2007). Structures under Crash and Impact, New York, Springer.

Hudson, G.E. (1951). "A theory of the dynamic plastic deformation of a thin diaphragm". Journal of Applied Physics, Vol. 22, pp. 1-11.

Hui, T. \& Oskay, C. (2012). "Computational modeling of polyurea-coated composites subjected to blast loads". Journal of Composite Materials, Vol. 46, pp. 21672178.

International Organization for Standardization (1975). ISO 2553:1975 - Standard Atmosphere. 
Johnson, C.F., Slawson, T.R., Cummins, T.K. \& Davis, J.L. (2005). "Concrete masonry unit walls retrofitted with elastomeric systems for blast loads". In: 24th Army Science Conference, Florida.

Johnson, G.R. \& Cook, W.H. (1983). "A constitutive model and data for metals subjected to large strains, high strain rates and high temperatures". In: Proceedings of the Seventh International Ballistics Symposium, The Hague. pp. 541-547.

Kim, H., Citron, J., Youssef, G., Navarro, A. \& Gupta, V. (2012). "Dynamic fracture energy of polyurea-bonded steel/E-glass composite joints". Mechanics of Materials, Vol. 45, pp. 10-19.

Knox, K.J., Hammons, M.I., Lewis, T.T. \& Porter, J.R. (2000). Polymer materials for structural retrofit, Force Protection Branch, Air Expeditionary Forces Technology Division, Air Force Research Laboratory, Tyndall AFB, Fla.

Lee, E.L., Hornig, H.C. \& Kury, J.W. (1968). Adiabatic expansion of high explosive detonation products, Lawrence Radiation Laboratory, University of California, Livermore.

Lee, G., Mock, W., Fedderly, J., Drotar, J., Balizer, E. \& Conner, M. (2007). "The effect of mechanical deformation on the glass transition temperature of polyurea". In: Proceedings of the Conference of the American Physical Society Topical Group on Shock Compression of Condensed Matter, 24-29 June 2007, Hawaii. pp. 711-714.

Li, C. \& Lua, J. (2009). "A hyper-viscoelastic constitutive model for polyurea". Materials Letters, Vol. 63, pp. 877-880.

Lu, J. (2011). The University of Melbourne, Personal communication.

Luccioni, B., Ambrosini, D. \& Danesi, R.F. (2006). "Blast Assessment using hydrocodes". Engineering Structures, Vol. 28, pp. 1736-1744.

Mac Donald, B.J. (2007). Practical stress analysis with finite elements, Dublin, Glasnevin Publishing.

Martin, J.B. \& Symonds, P.S. (1966). "Mode approximations for impulsively loaded rigid-plastic structures". In: Proceedings of the ASCE 92 (EMS). pp. 43-46.

Matthews, W. (2004). Services test spray-on vehicle armor. Army Times. Vancouver, USA. 
McShane, G.J., Stewart, C., Aronson, M.T., Wadley, H.N.G., Fleck, N.A. \& Deshpande, V.S. (2008). "Dynamic rupture of polymer-metal bilayer plates". International Journal of Solids and Structures, Vol. 45, pp. 4407-4426.

Menkes, S.B. \& Opat, H.J. (1973). "Broken beams". Experimental Mechanics, Vol. 13, pp. 480-486.

MIL-S-22698C (1988). U.S. Military Specification: Steel plate, shapes and bars, weldable ordinary strength and higher strength: structural, 29 June 1988.

Needham, C.E. (2010). Blast Waves, Heidelberg, Springer.

Nurick, G.N., Pearce, H.T. \& Martin, J.B. (1987). "Predictions of transverse deflections and in-plane strains in impulsively loaded thin plates". International Journal of Mechanical Sciences, Vol. 29, pp. 435-442.

Nurick, G.N. \& Shave, G.C. (1996). "The deformation and tearing of thin square plates subjected to impulsive loads - an experimental study". International Journal of Impact Engineering, Vol. 18, pp. 99-116.

Ogden, R.W. (1972). "Large deformation isotropic elasticity - on the correlation of theory and experiment for incompressible rubberlike solids". Proceedings of the Royal Society of London. Series A. Mathematical and Physical Sciences, Vol. 326, pp. 565-584.

Olsen, M.D., Nurick, G.N. \& Fagnan, J.R. (1993). "Deformation and rupture of blast loaded square plates-predictions and experiments". International Journal of Impact Engineering, Vol. 13, pp. 279-291.

Operation Enduring Freedom. (2012). iCasualties.org [Online]. Available: http://icasualties.org/OEF/index.aspx [Accessed 3 December 2012].

Qiao, J., Amirkhizi, A.V., Schaaf, K., Nemat-Nasser, S. \& Wu, S. (2011). "Dynamic mechanical and ultrasonic properties of polyurea". Mechanics of Materials, Vol. 43, pp. 598-607.

Ramajeyathilagam, K. \& Vendhan, C.P. (2004). "Deformation and rupture of thin rectangular plates subjected to underwater shock". International Journal of Impact Engineering, Vol. 30, pp. 699-719.

Raman, S.N. (2011). Polymeric coatings for enhanced protection of reinforced concrete structures from the effects of blast. PhD Thesis, The University of Melbourne. 
Richardson, J.M. \& Kirkwood, J.G. (1950). "Theory of the plastic deformation of thin plates by underwater explosions". Underwater Explosion Research, Vol. 3, The Damage Process, Office of Naval Research, pp. 305-421. .

Ritzel, D. (2007). Short course: Basics of blast physics, damage and injury course notes. November - December 2007, Melbourne: Dyn-Fx Consulting Ltd.

Roland, C.M. \& Casalini, R. (2007). "Effect of hydrostatic pressure on the viscoelastic response of polyurea". Polymer, Vol. 48, pp. 5747-5752.

Roland, C.M., Twigg, J.N., Vu, Y. \& Mott, P.H. (2007). "High strain rate mechanical behavior of polyurea". Polymer, Vol. 48, pp. 574-578.

Sarva, S.S., Deschanel, S., Boyce, M.C. \& Chen, W. (2007). "Stress - strain behavior of a polyurea and a polyurethane from low to high strain rates". Polymer, Vol. 48, pp. 2208-2213.

Shim, J. \& Mohr, D. (2011). "Rate dependent finite strain constitutive model of polyurea". International Journal of Plasticity, Vol. 27, pp. 868-886.

Sun, E.Q. (2006). "Shear locking and hourglassing in MSC Nastran, ABAQUS and ANSYS". In: MSC Software Corporation's 2006 Americas Virtual Product Development Conference, California.

Symonds, P.S. \& Chon, C.T. (1979). "Finite viscoplastic deflections of an impulsively loaded plate by the mode approximation technique". Journal of the Mechanics and Physics of Solids, Vol. 27, pp. 115-133.

Taylor, G.I. (1950). "The distortion under pressure of a diaphragm which is clamped along its edge and stressed beyond its elastic limit". Underwater Explosion Research, Vol. 3, The Damage Process, Office of Naval Research, pp. 107121.

Teeling-Smith, R.G. \& Nurick, G.N. (1991). "The deformation and tearing of thin circular plates subjected to impulsive loads". International Journal of Impact Engineering, Vol. 11, pp. 77-91.

Tekalur, S.A., Shulka, A. \& Shivakumar, K. (2008). "Blast resistance of polyurea based layered composite materials". Composite Structures, Vol. 84, pp. 271281.

US Army Technical Report (1986). Fundamentals of protective design for conventional weapons, TM-5-855-1, Washington DC.

Walter, P.L. (2010). Measuring Static Overpressures in Air Blast Environments, Technical Note, TN-27, PCB Piezotronics, USA. 
Wierzbicki, T. (1999). "Petalling of plates under explosive and impact loading". International Journal of Impact Engineering, Vol. 22, pp. 935-954.

Wierzbicki, T. \& Nurick, G.N. (1996). "Large deformation of thin plates under localised impulsive loading". International Journal of Impact Engineering, Vol. 18, pp. 899-918.

Xue, L., Mock, W.J. \& Belytschko, T. (2010). "Penetration of DH-36 steel plates with and without polyurea coating". Mechanics of Materials, Vol. 42, pp. 9811003.

Xue, Z. \& Hutchinson, J.W. (2007). "Neck retardation and enhanced energy absorption in metal-elastomer bilayers". Mechanics of Materials, Vol. 39, pp. 473-487.

Yi, J., Boyce, M.C., Lee, G.F. \& Balizer, E. (2006). "Large deformation ratedependent stress-strain behavior of polyurea and polyurethanes". Polymer, Vol. 47, pp. 319-329.

Zukas, A. (2004). Introduction to Hydrocodes, Amsterdam, Elsevier. 


\section{Publications Originating from this Thesis}

Ackland, K., Anderson, C. \& Ngo, T. (2013). "Deformation of polyurea coated steel plates under localised blast loading". International Journal of Impact Engineering, Vol. 51, pp. 13-22.*

Ackland, K., Anderson, C. \& St John, N. (2007). "Polymeric coatings for enhanced protection of structures from the explosive effects of blast". In: Mendis, P., Lai, J., Dawson, E. \& Abbass, H., eds. Recent advances in security technology 2007, Proceedings of the RNSA Security Technology Conference, 28 September 2007, Melbourne, Australia. Australian Homeland Security Research Centre, pp. 90-96.

Ackland, K., Lu, J. \& Ngo, T. (2009). "Numerical simulation of blast loading on structures using AUTODYN and LS-DYNA". In: Shock and Impact Loads on Structures, Adelaide, Australia. pp. 113-118.

Ackland, K. \& St John, N. (2011). Experimental and numerical investigations into polymeric coatings for blast protection, DSTO Technical Report, DSTO-TR2591, Melbourne, Defence Science and Technology Organisation.

Ackland, K., Warne, S. \& Anderson, C. (2010). "Blast loading of metallic plates with polyurea coatings". In: Proceedings of the 21st Australasian Conference on the Mechanics of Structures and Materials, Melbourne, Australia.

* This paper is included as Appendix F 


\section{Appendix A Blast Rig Drawings}

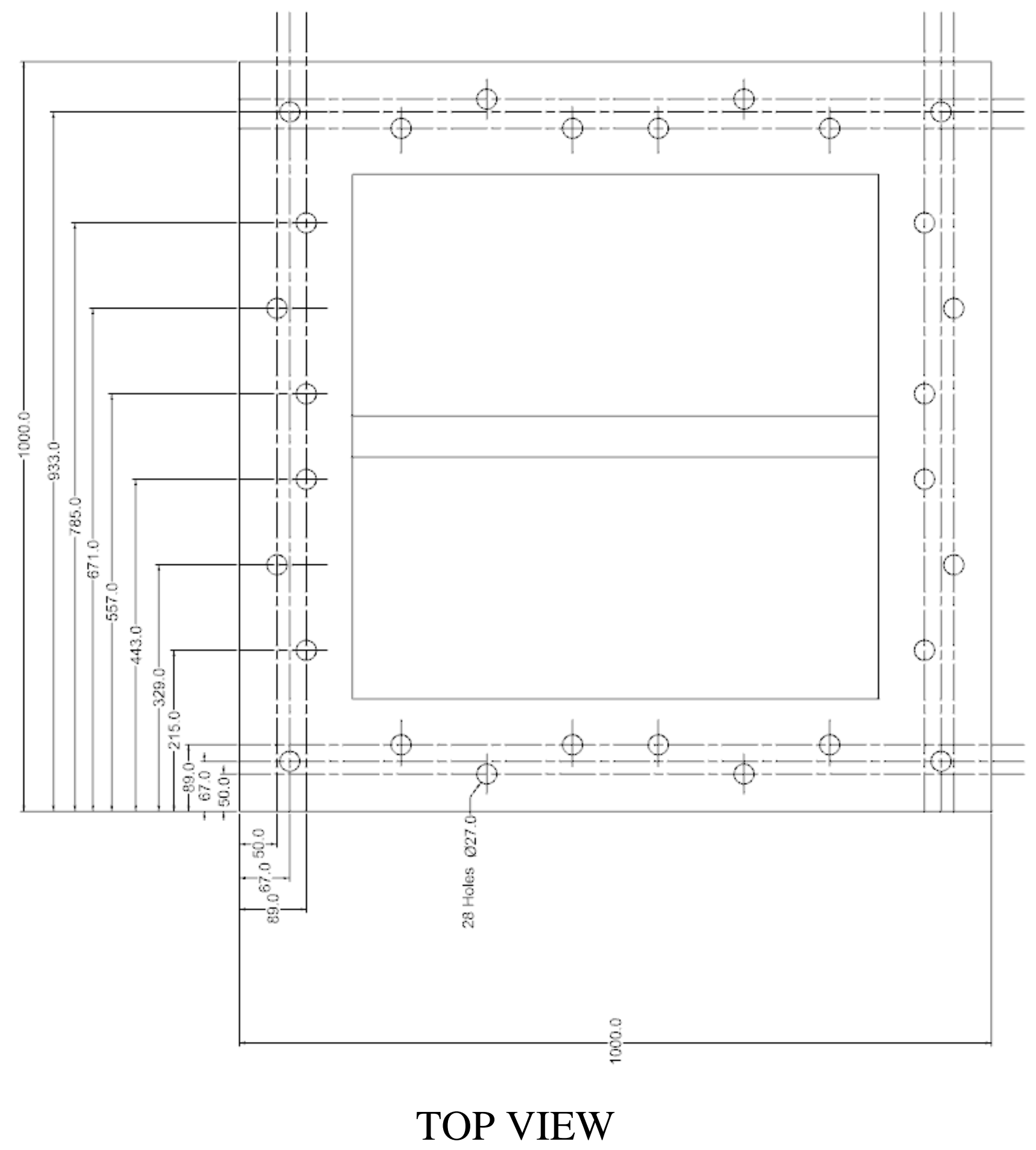


Appendix A. Blast Rig Drawings.

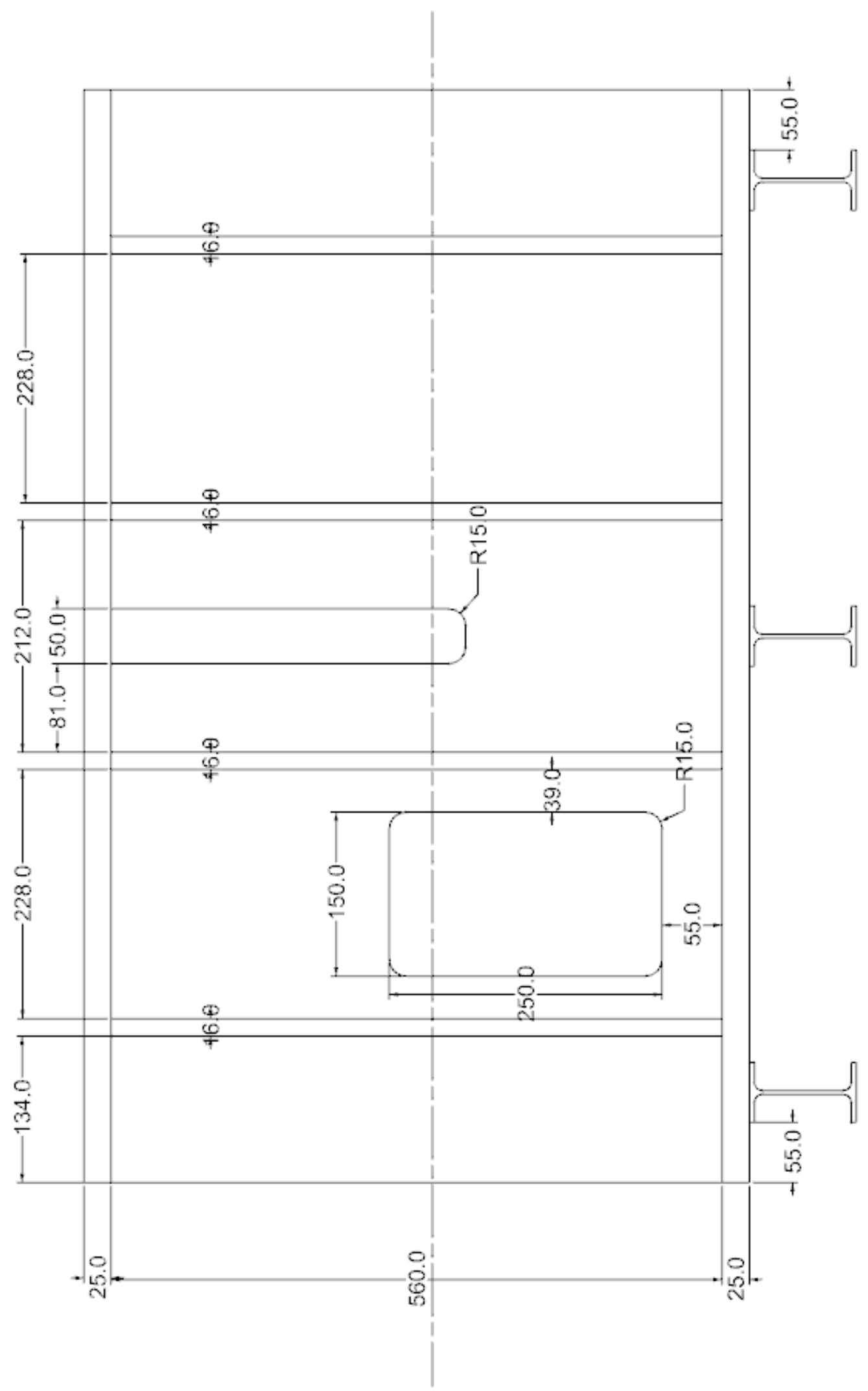

SIDE VIEW 
Appendix A. Blast Rig Drawings.

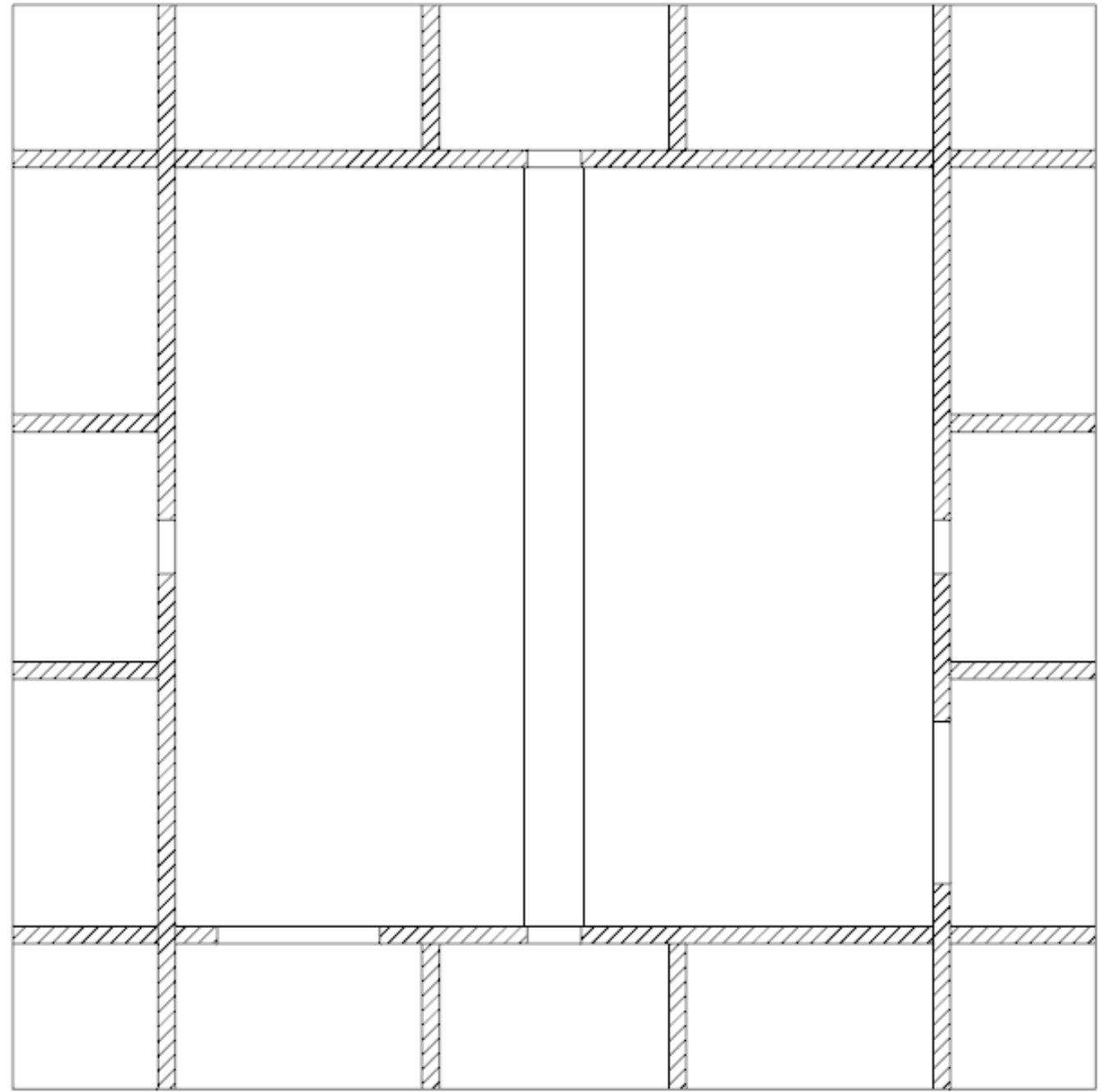

Section A-A 


\section{Appendix B Pressure Gauge Results}

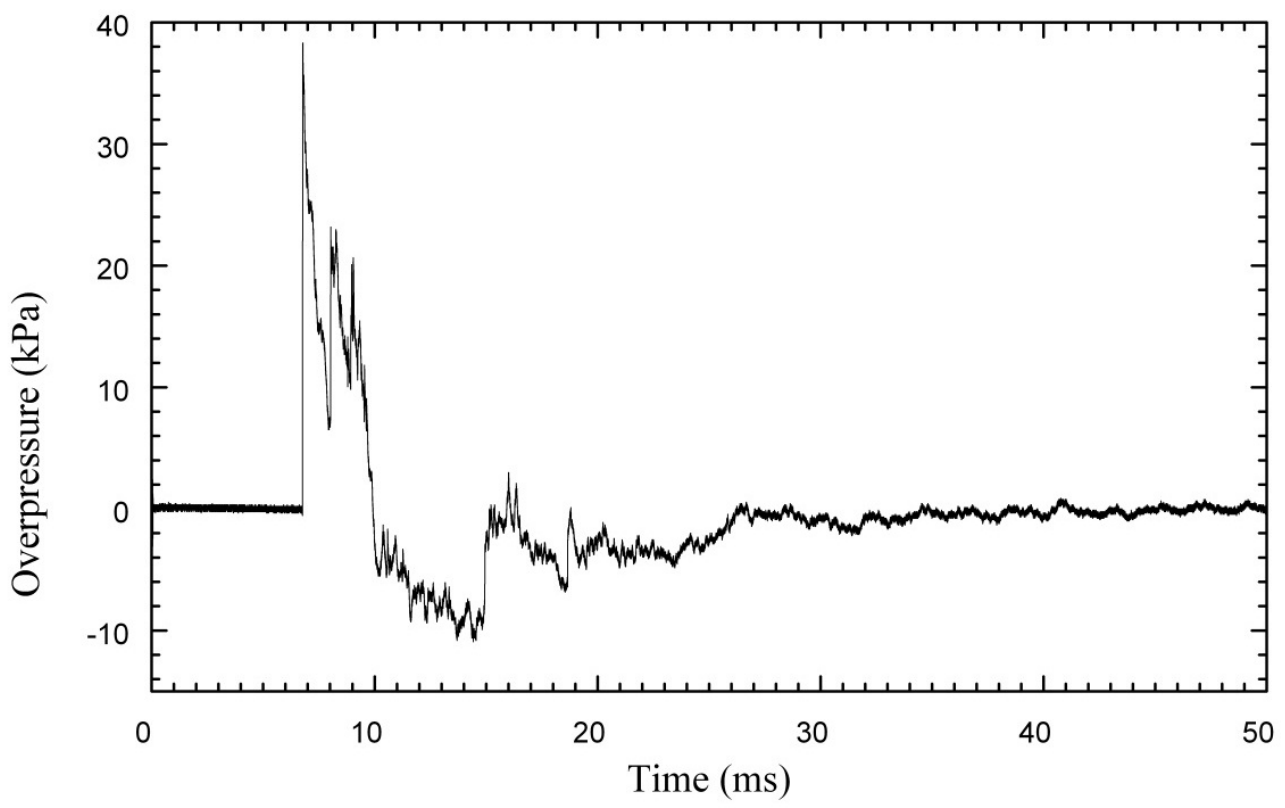

Figure B.1 Pressure trace for Event 1, gauge P1, located $1 \mathrm{~m}$ above the ground and $4 \mathrm{~m}$ from the charge centre.

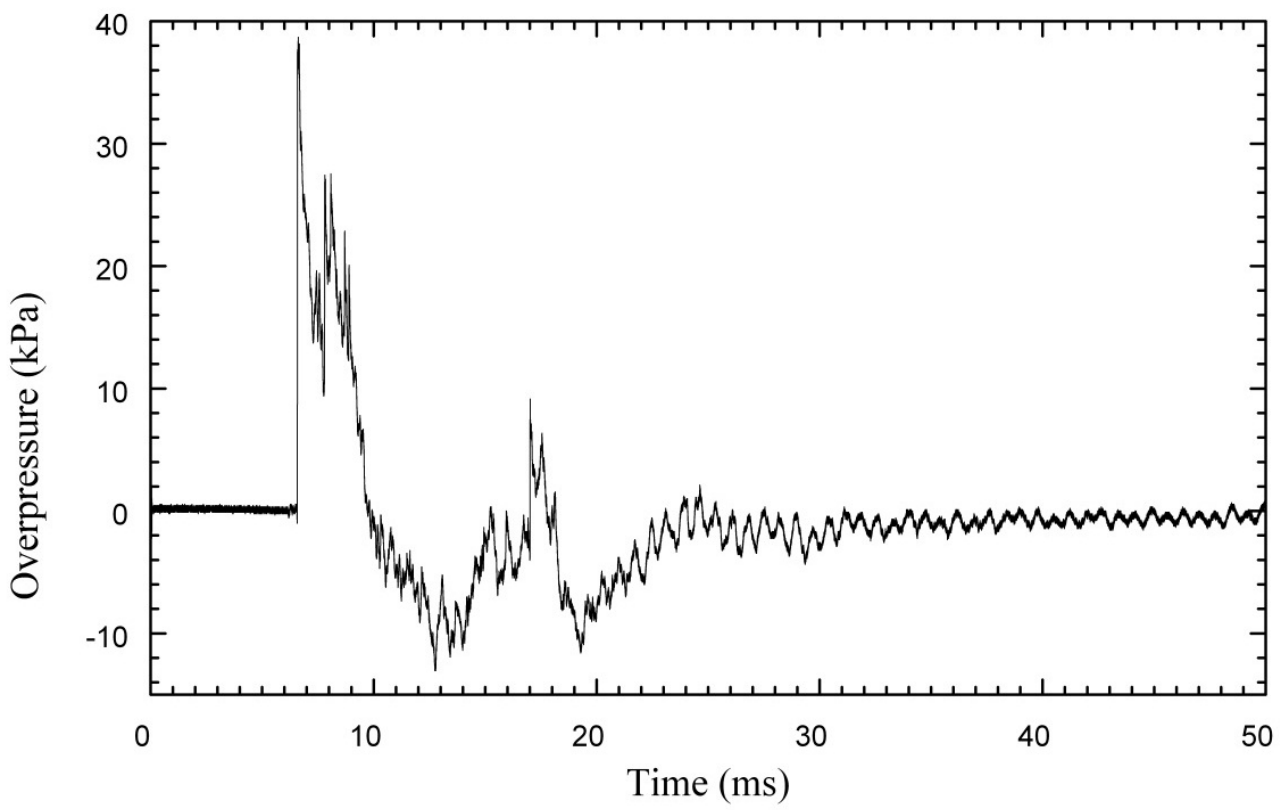

Figure B.2 Pressure trace for Event 1, gauge P2, located $1 \mathrm{~m}$ above the ground and $4 \mathrm{~m}$ from the charge centre. 
Appendix B. Pressure Gauge Results.

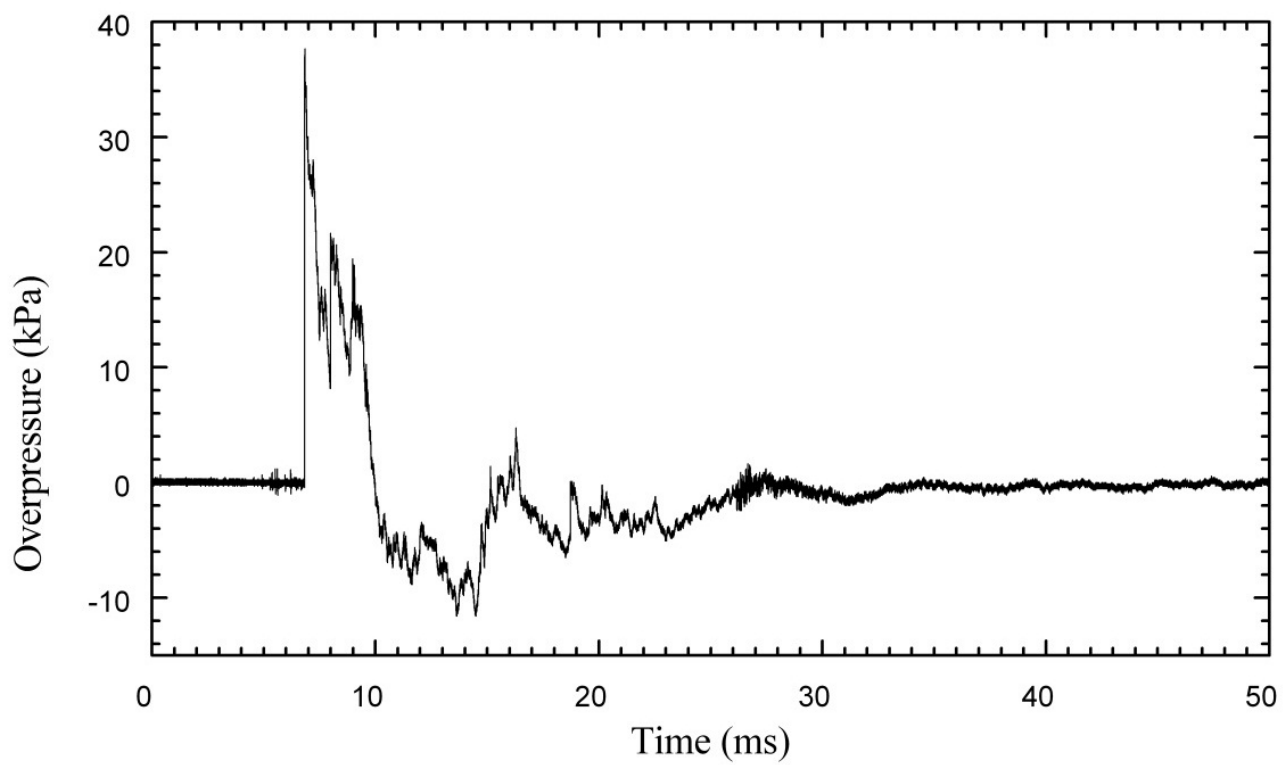

Figure B.3 Pressure trace for Event 2, gauge P1, located $1 \mathrm{~m}$ above the ground and $4 \mathrm{~m}$ from the charge centre.

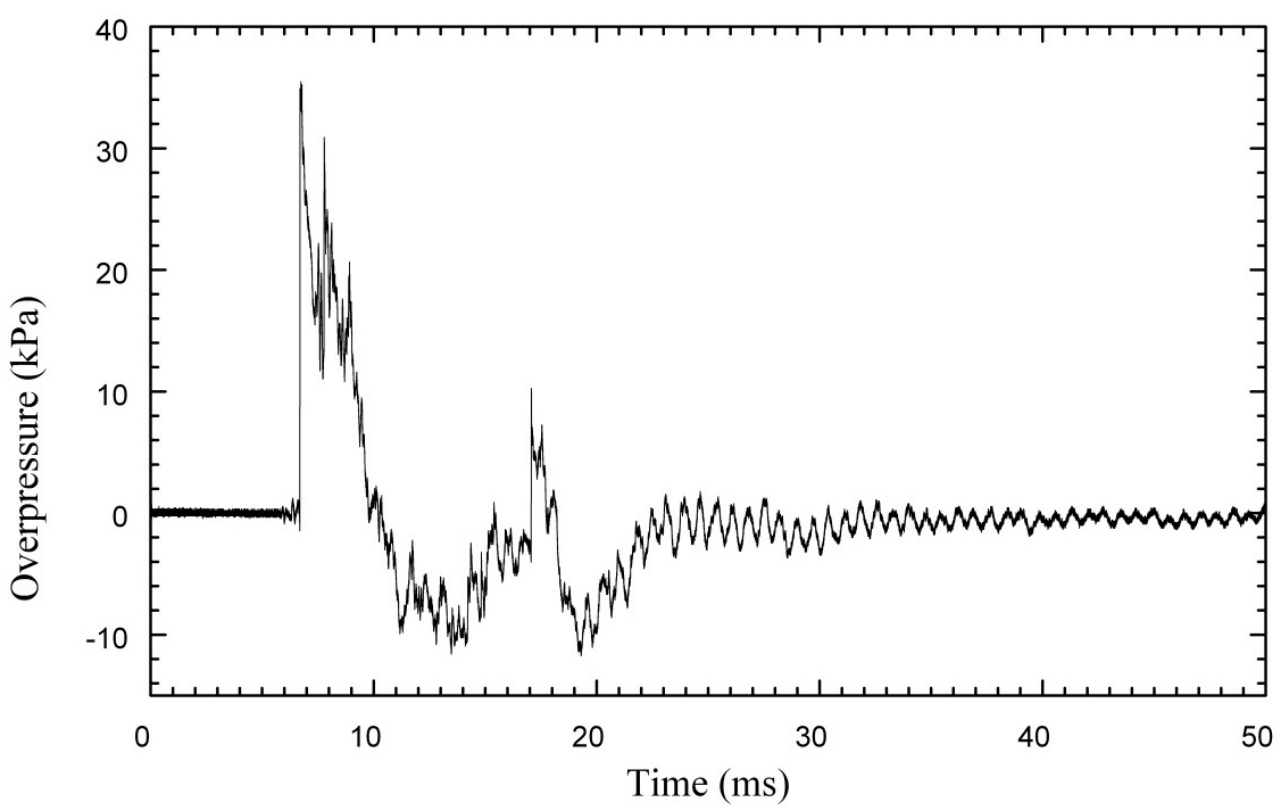

Figure B.4 Pressure trace for Event 2, gauge P2, located $1 \mathrm{~m}$ above the ground and $4 \mathrm{~m}$ from the charge centre. 
Appendix B. Pressure Gauge Results.

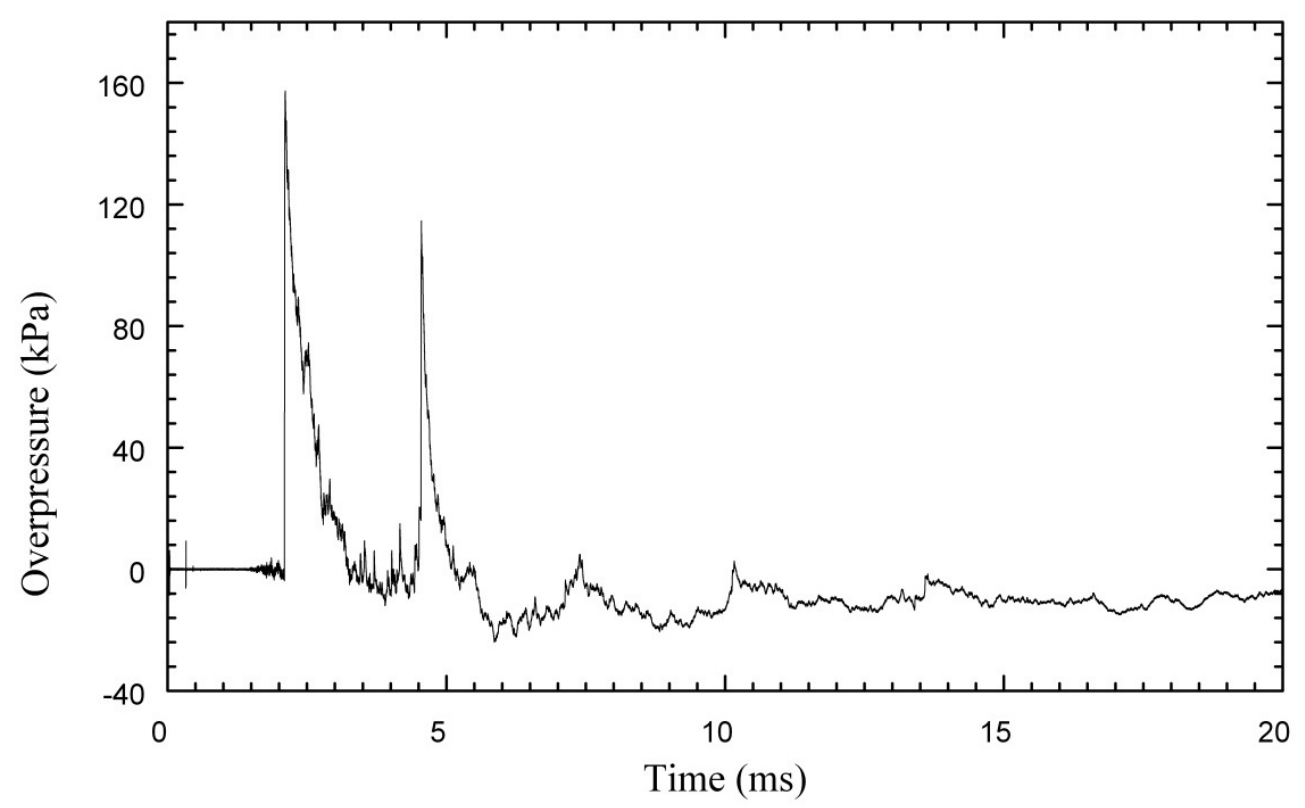

Figure B.5 Pressure trace for Event 3, gauge P1, located $1 \mathrm{~m}$ above the ground and $2 \mathrm{~m}$ from the charge centre.

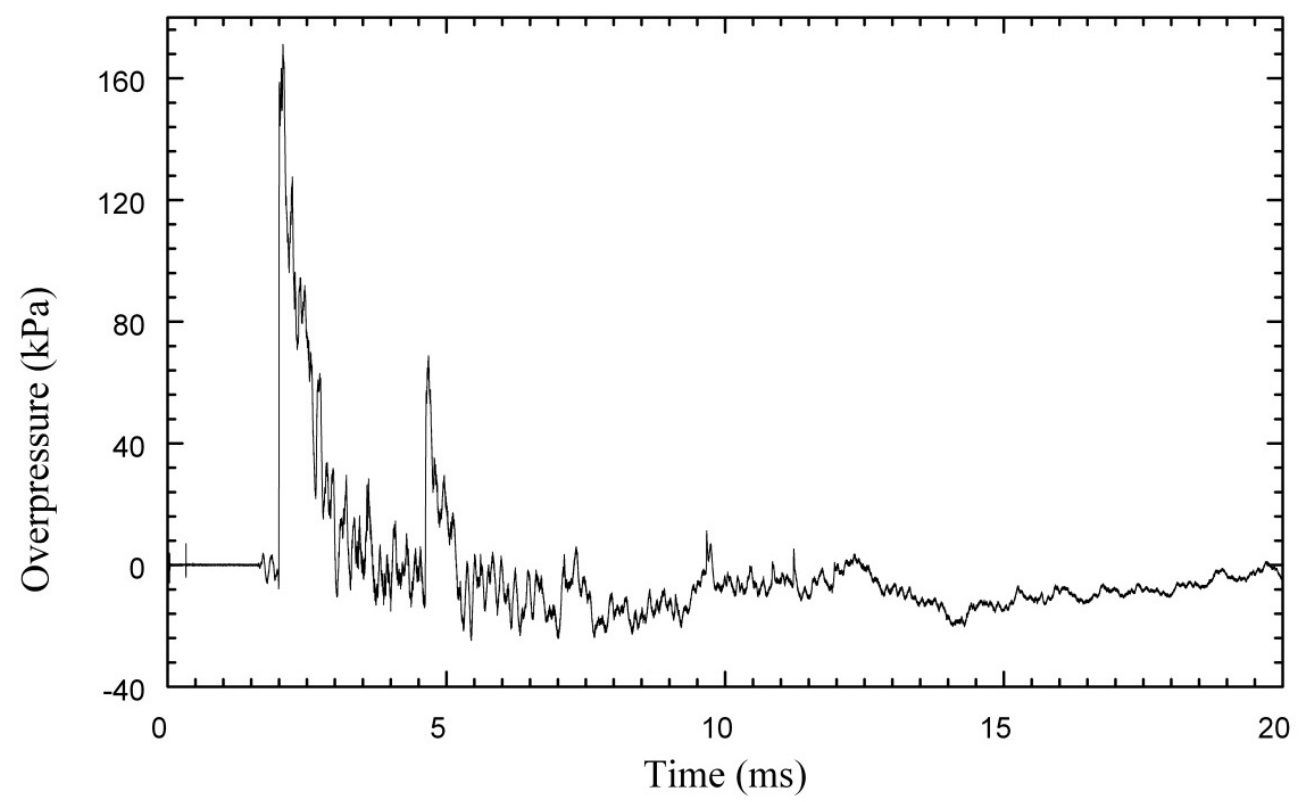

Figure B.6 Pressure trace for Event 3, gauge P2, located $1 \mathrm{~m}$ above the ground and $2 \mathrm{~m}$ from the charge centre. 
Appendix B. Pressure Gauge Results.

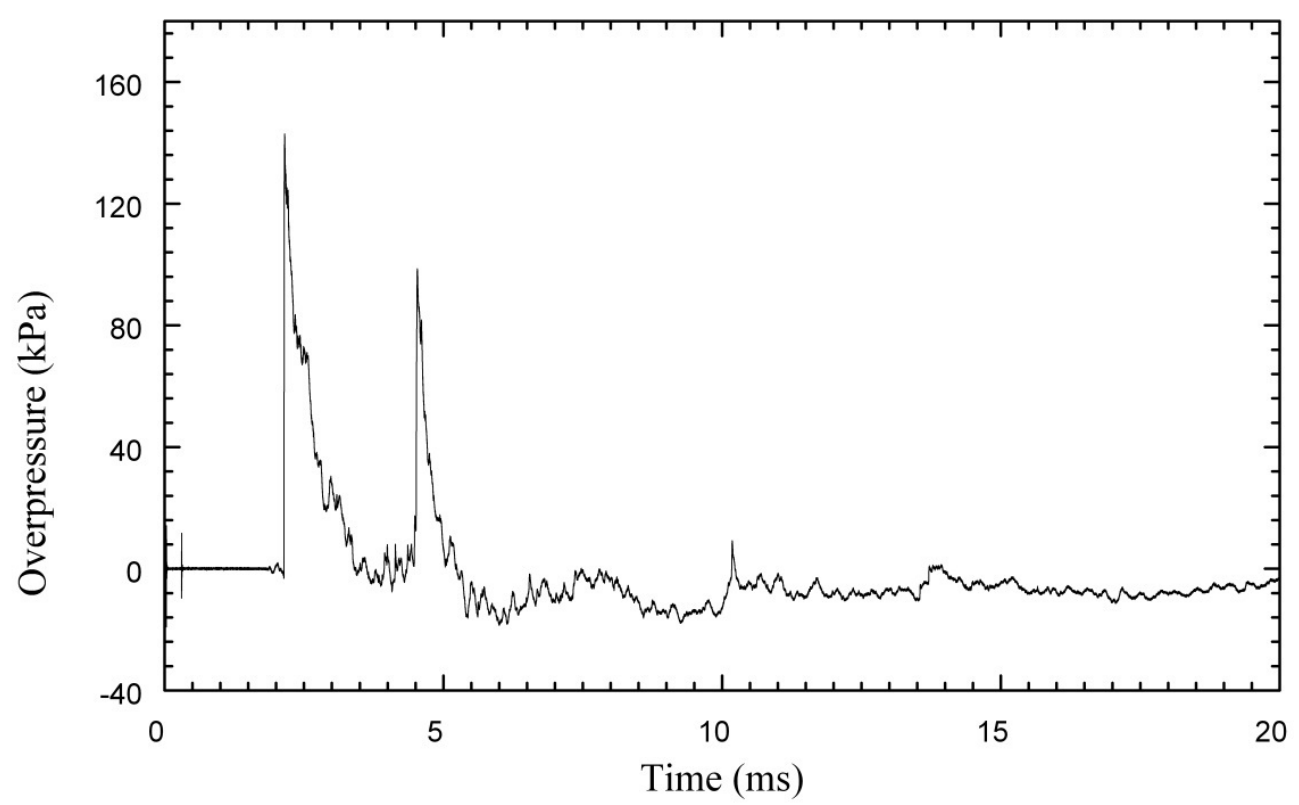

Figure B.7 Pressure trace for Event 4, gauge P1, located $1 \mathrm{~m}$ above the ground and $2 \mathrm{~m}$ from the charge centre.

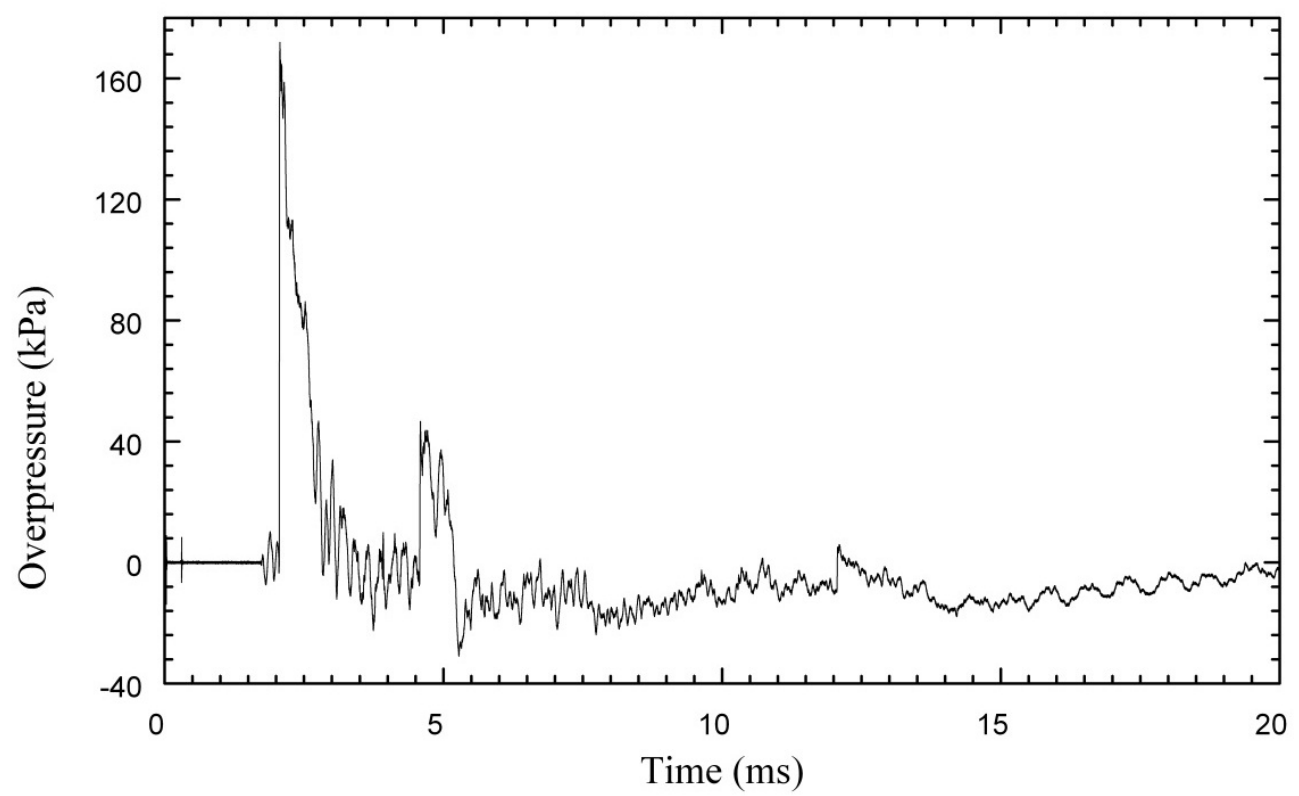

Figure B.8 Pressure trace for Event 4, gauge P2, located $1 \mathrm{~m}$ above the ground and $2 \mathrm{~m}$ from the charge centre. 
Appendix B. Pressure Gauge Results.

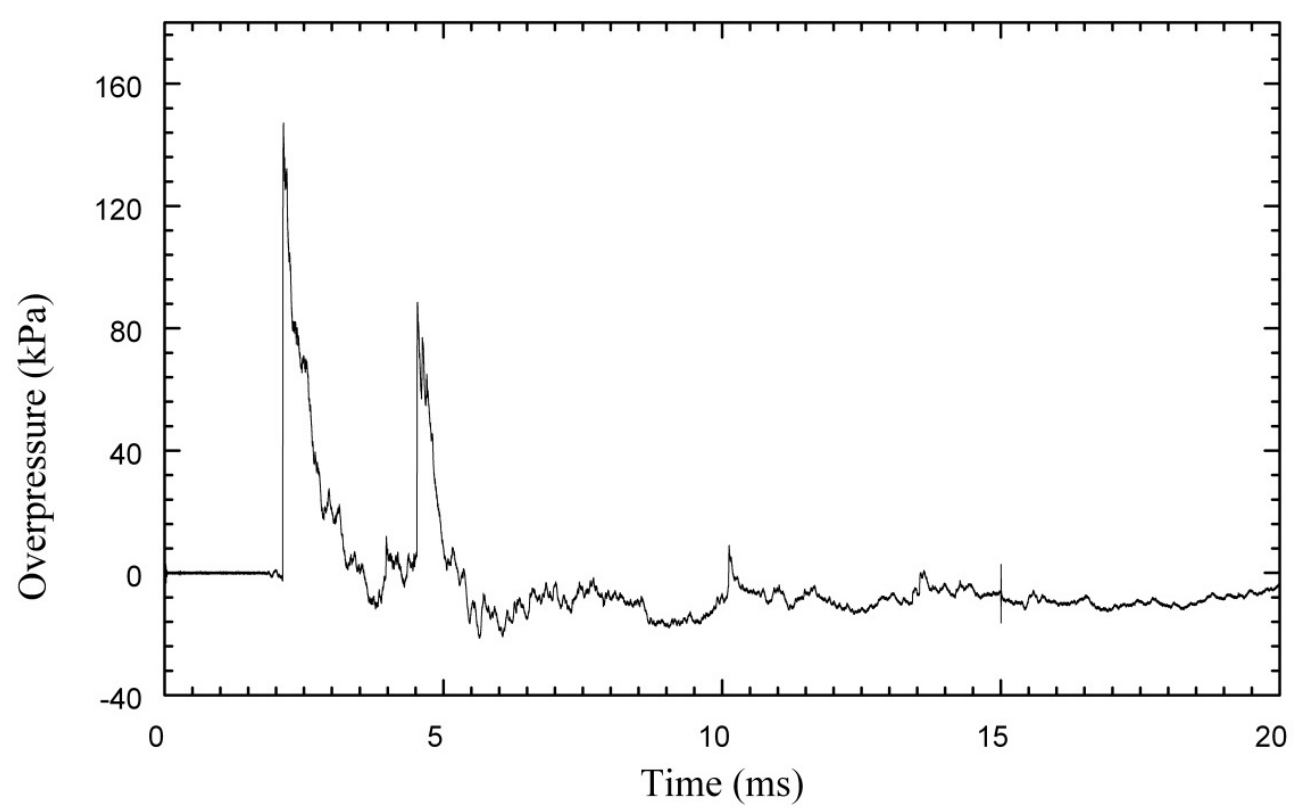

Figure B.9 Pressure trace for Event 5, gauge P1, located $1 \mathrm{~m}$ above the ground and $2 \mathrm{~m}$ from the charge centre.

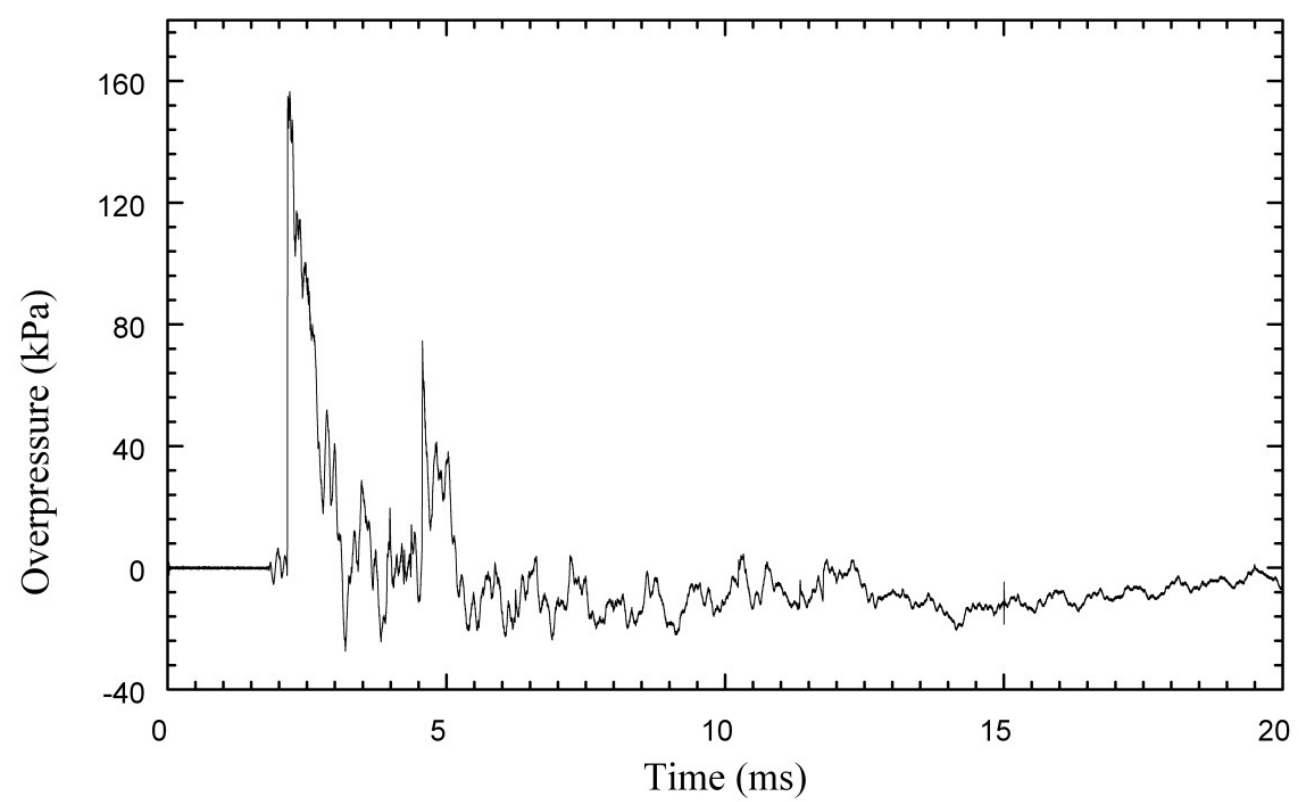

Figure B.10 Pressure trace for Event 5, gauge P1, located $1 \mathrm{~m}$ above the ground and $2 \mathrm{~m}$ from the charge centre. 
Appendix B. Pressure Gauge Results.

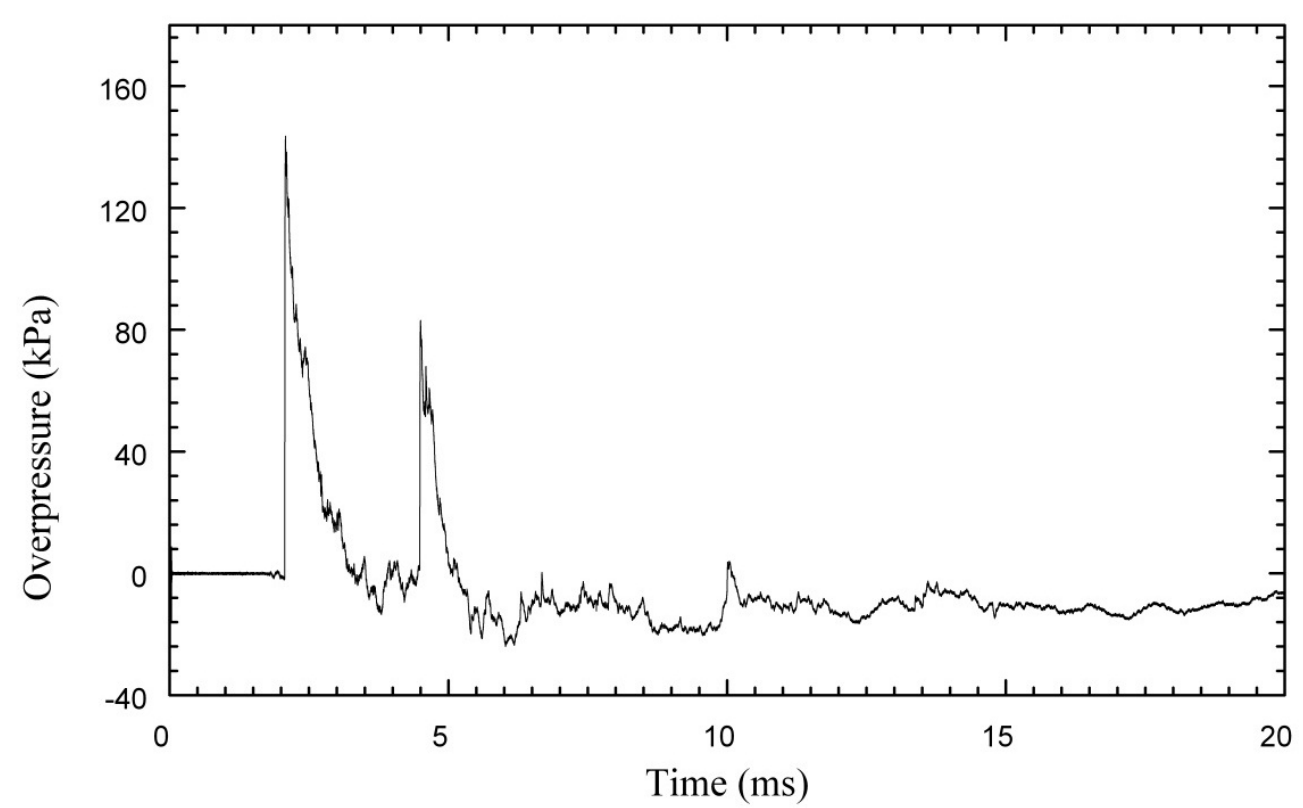

Figure B.11 Pressure trace for Event 6, gauge P1, located $1 \mathrm{~m}$ above the ground and $2 \mathrm{~m}$ from the charge centre.

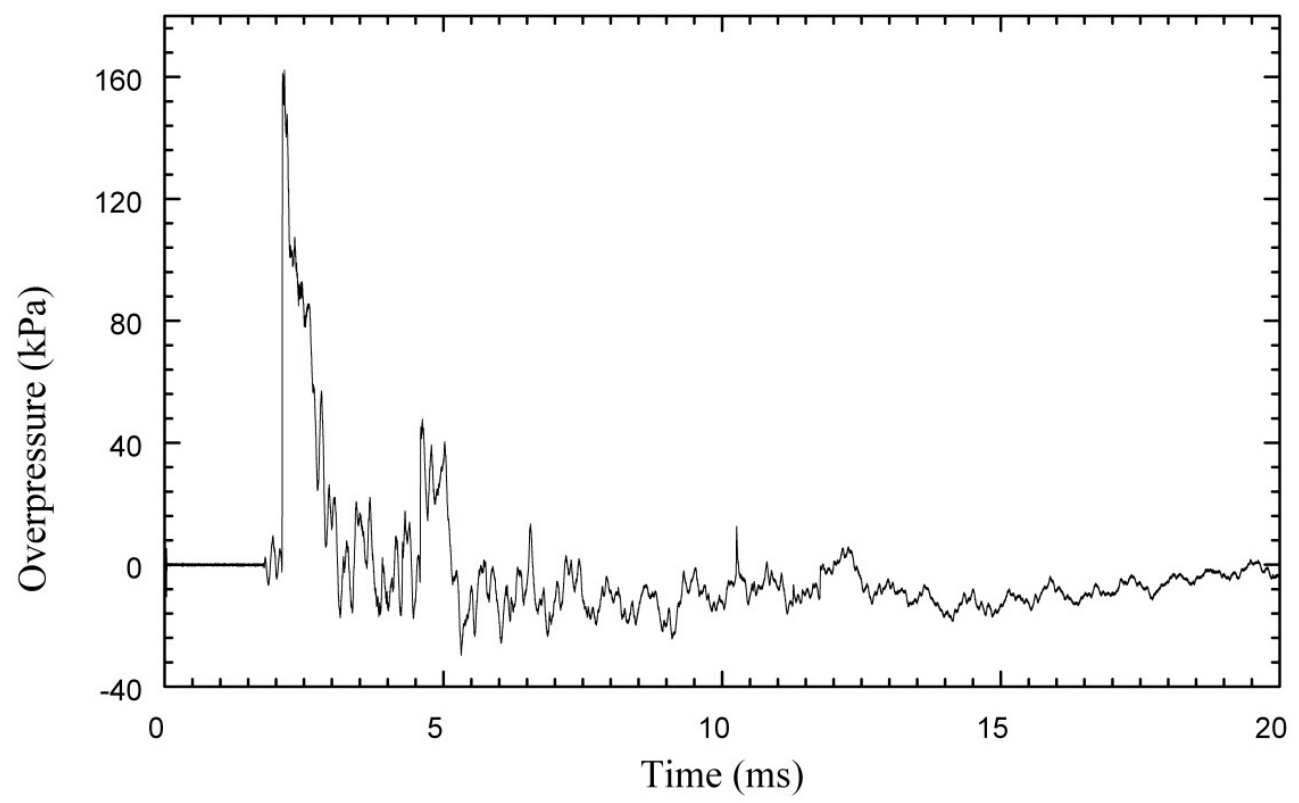

Figure B.12 Pressure trace for Event 6, gauge P2, located $1 \mathrm{~m}$ above the ground and $2 \mathrm{~m}$ from the charge centre. 
Appendix B. Pressure Gauge Results.

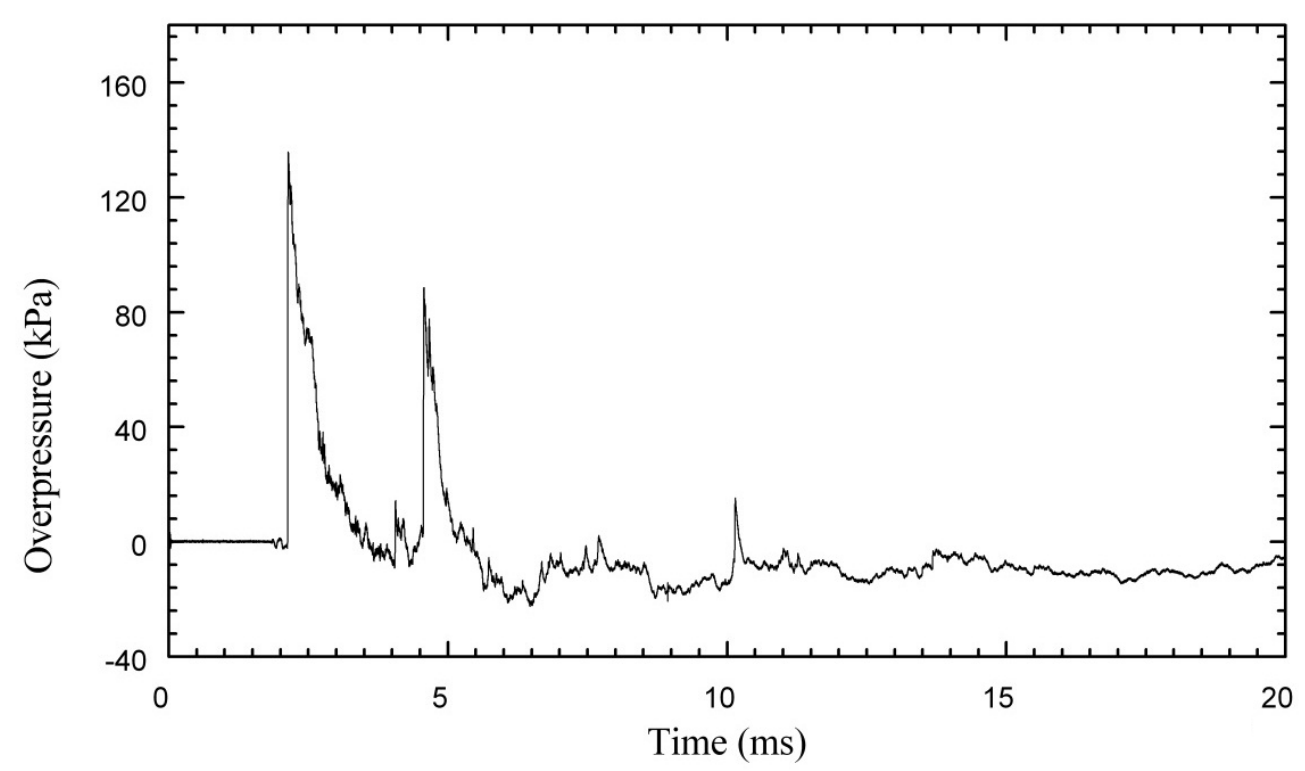

Figure B.13 Pressure trace for Event 7, gauge P1, located $1 \mathrm{~m}$ above the ground and $2 \mathrm{~m}$ from the charge centre.

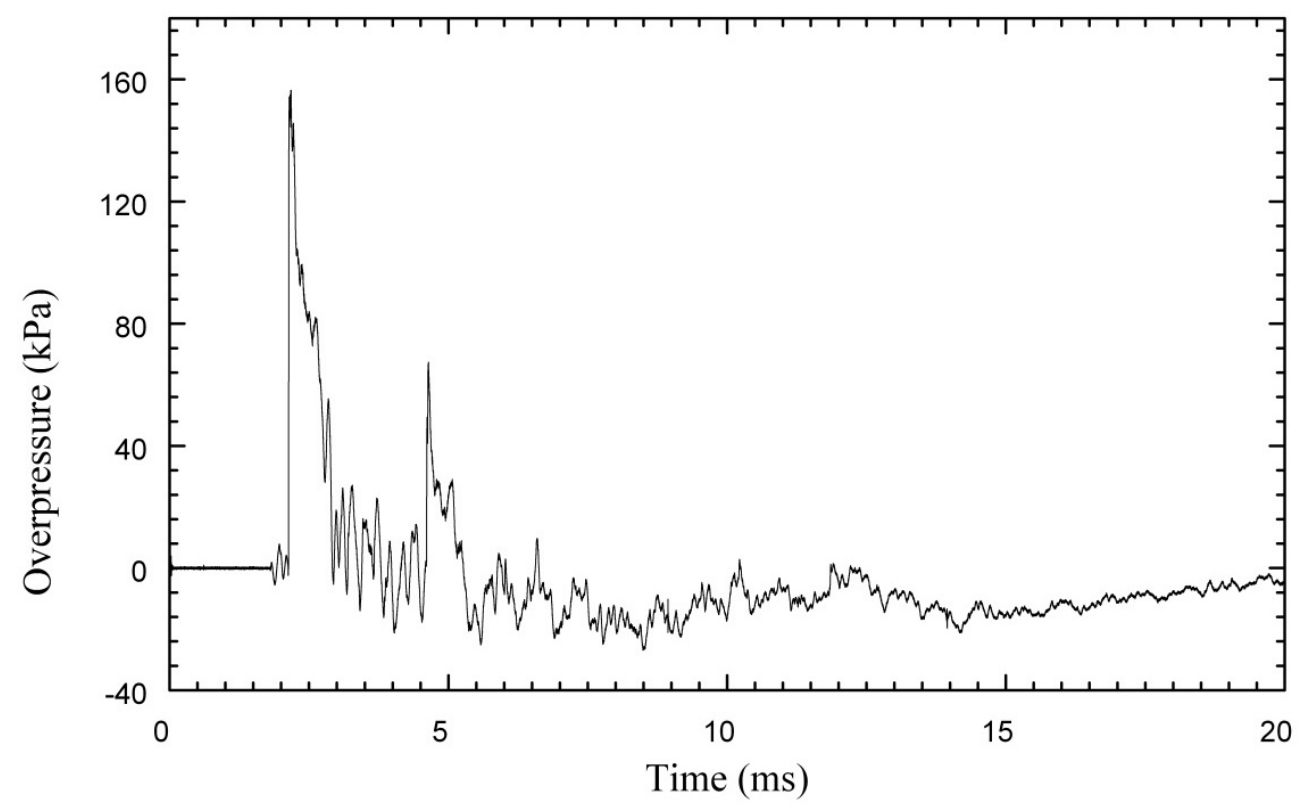

Figure B.14 Pressure trace for Event 7, gauge P2, located $1 \mathrm{~m}$ above the ground and $2 \mathrm{~m}$ from the charge centre. 
Appendix B. Pressure Gauge Results.

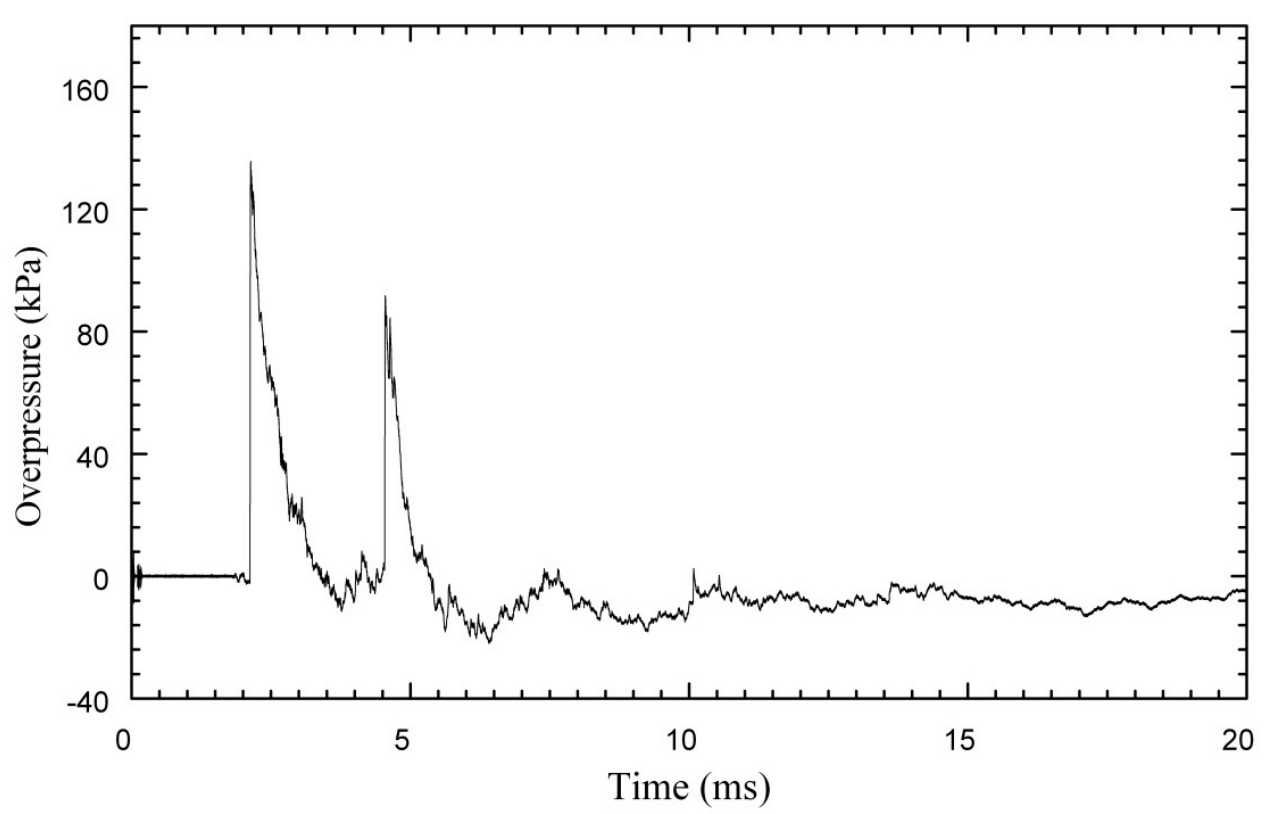

Figure B.15 Pressure trace for Event 8, gauge P1, located $1 \mathrm{~m}$ above the ground and $2 \mathrm{~m}$ from the charge centre.

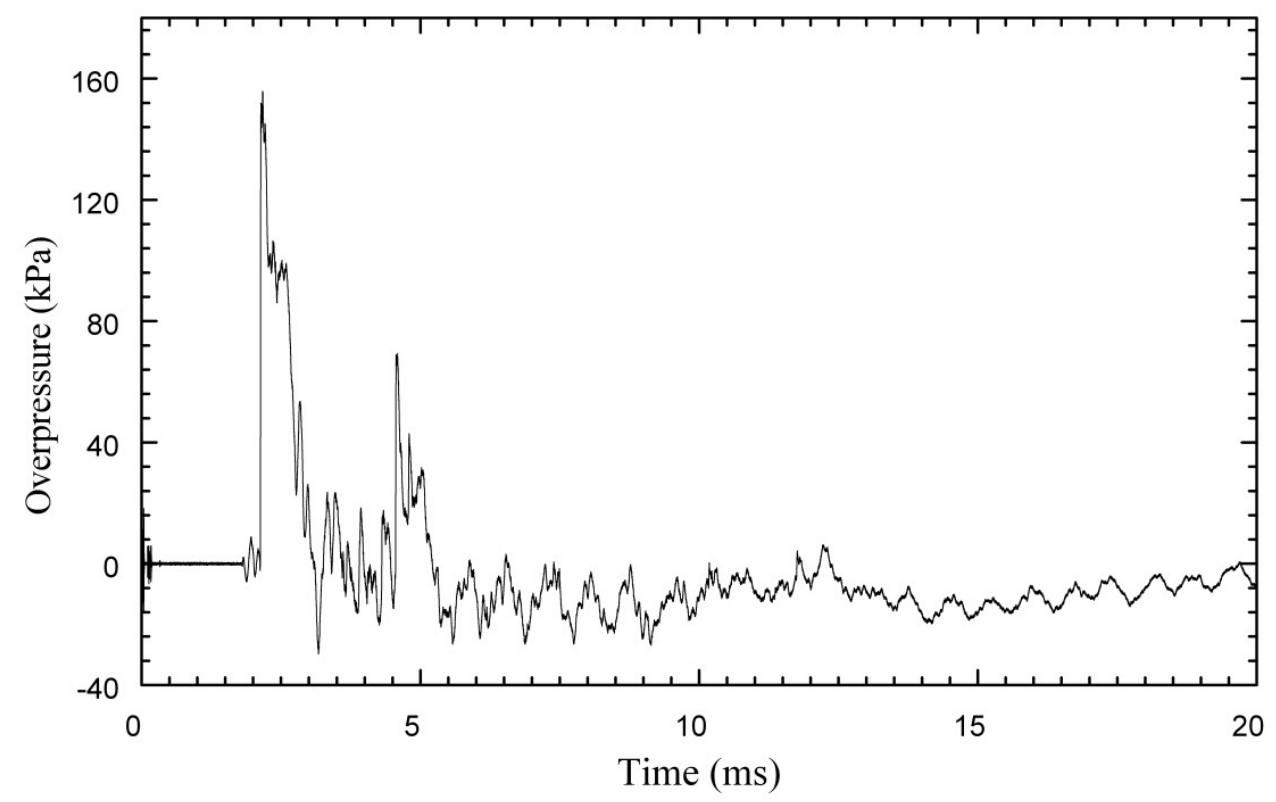

Figure B.16 Pressure trace for Event 8, gauge P2, located $1 \mathrm{~m}$ above the ground and $2 \mathrm{~m}$ from the charge centre. 
Appendix B. Pressure Gauge Results.

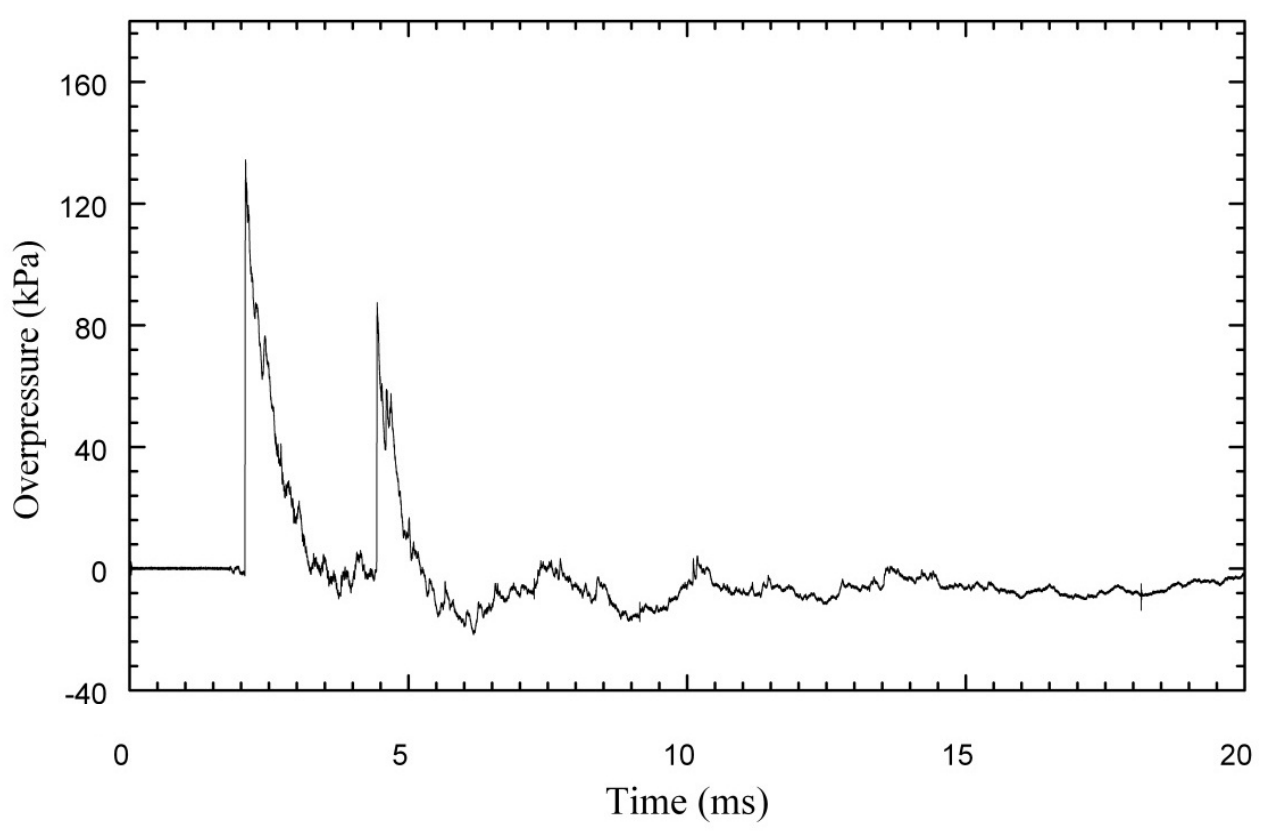

Figure B.17 Pressure trace for Event 9, gauge P1, located $1 \mathrm{~m}$ above the ground and $2 \mathrm{~m}$ from the charge centre.

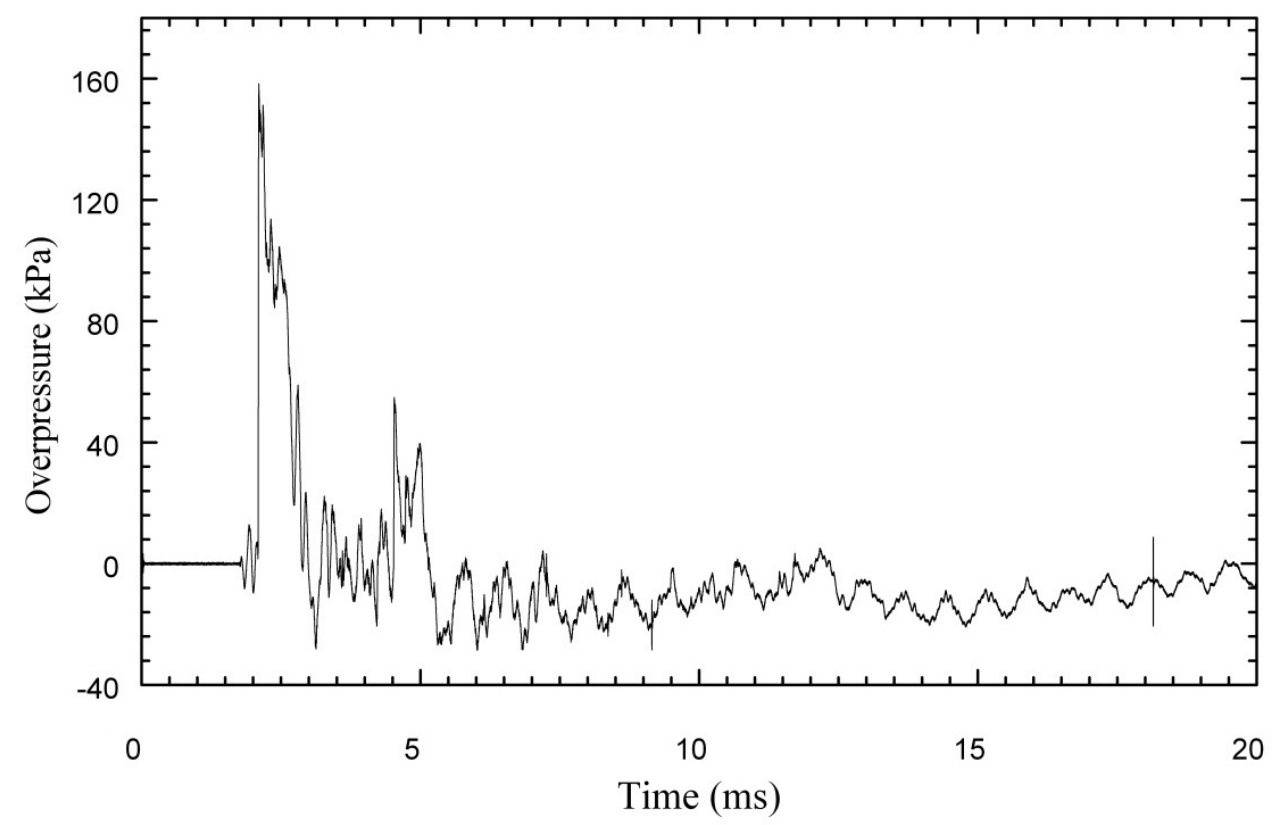

Figure B.18 Pressure trace for Event 9, gauge P2, located $1 \mathrm{~m}$ above the ground and $2 \mathrm{~m}$ from the charge centre. 
Appendix B. Pressure Gauge Results.

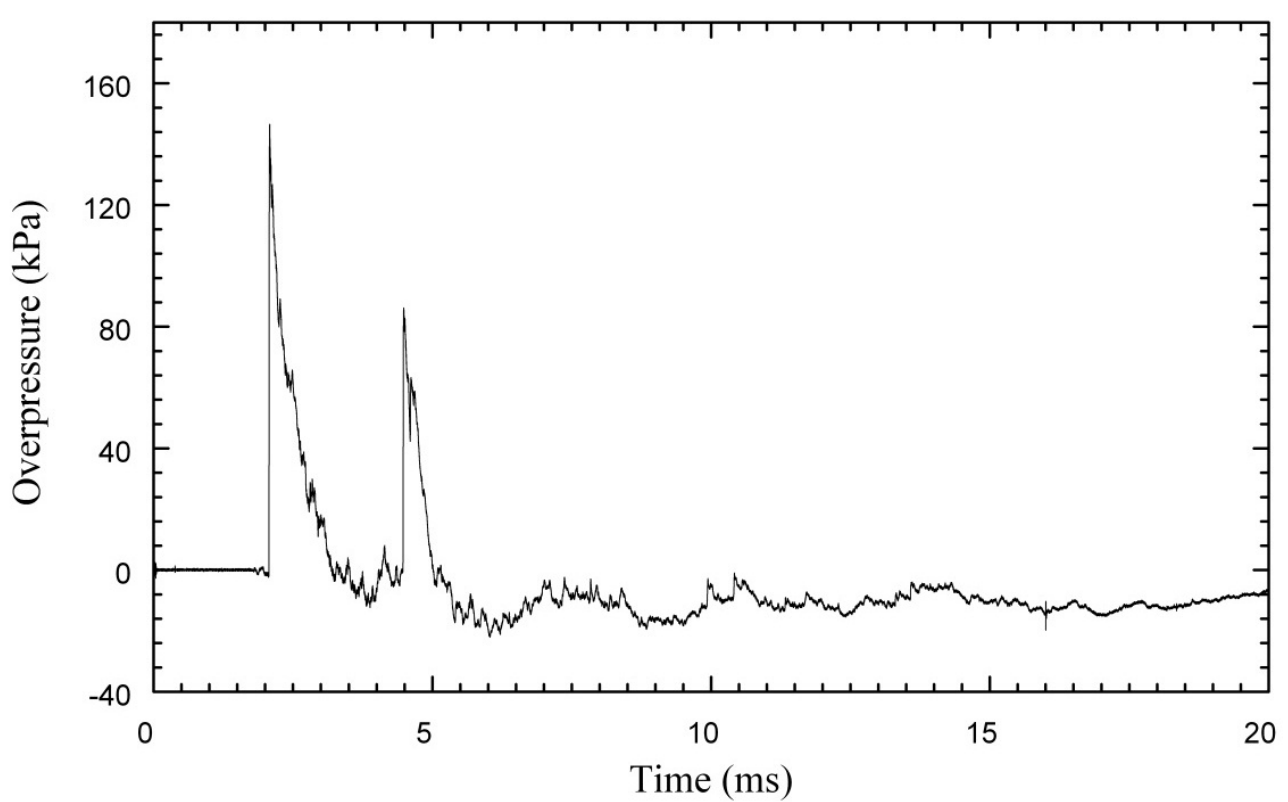

Figure B.19 Pressure trace for Event 10, gauge P1, located $1 \mathrm{~m}$ above the ground and $2 \mathrm{~m}$ from the charge centre.

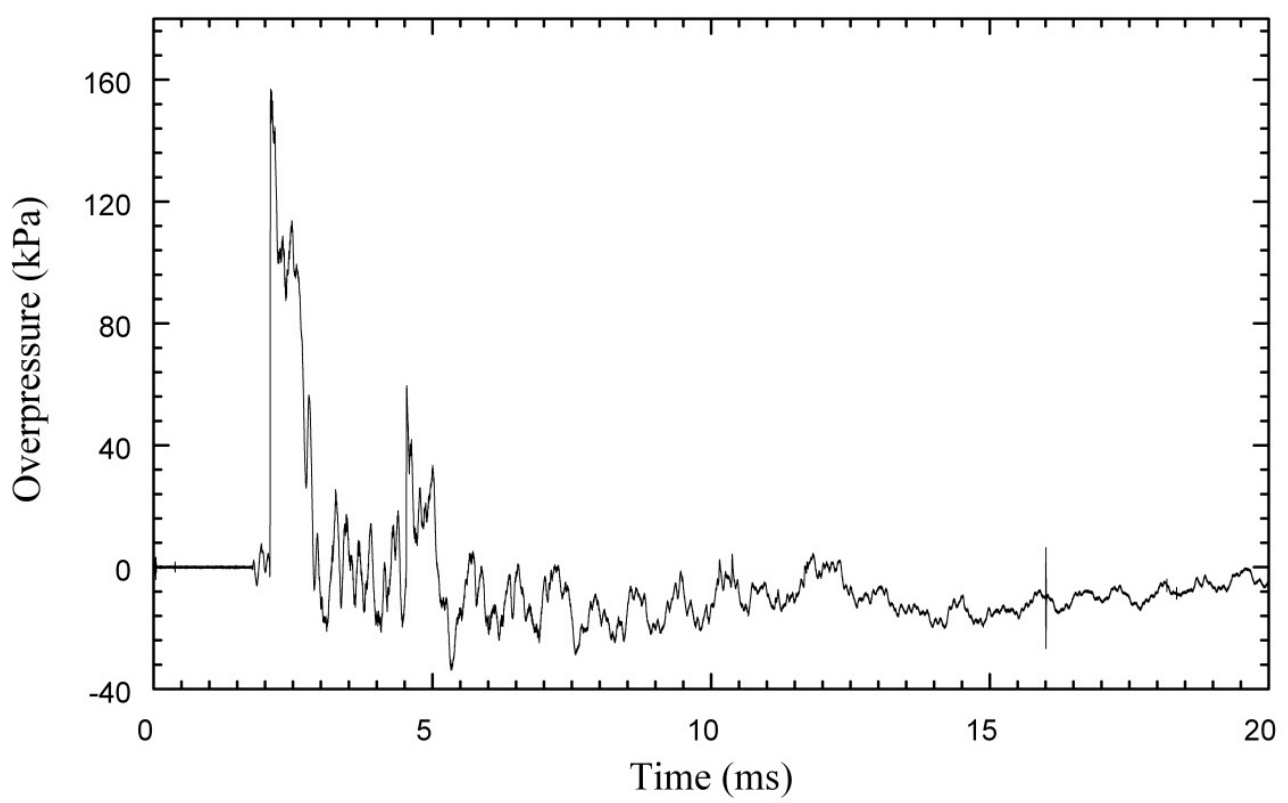

Figure B.20 Pressure trace for Event 10, gauge P2, located $1 \mathrm{~m}$ above the ground and $2 \mathrm{~m}$ from the charge centre. 


\section{Appendix C Plate Profiles Measured Using the 3D Laser Scanner}

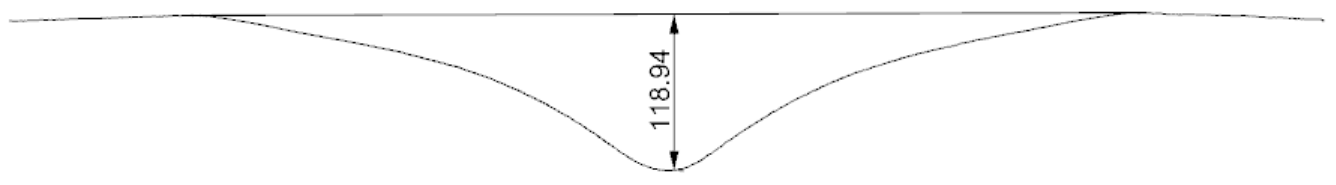

Figure C.1 Post-event deformation profile for Event 1, Plate B1. The dimension is in $\mathrm{mm}$.

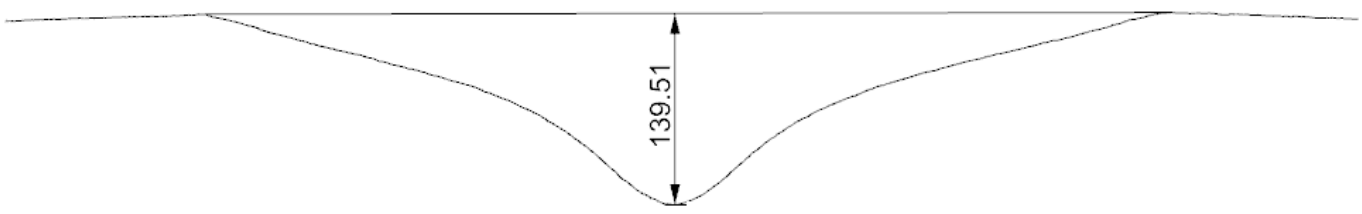

Figure C.2 Post-event deformation profile for Event 2, Plate D1. The dimension is in $\mathrm{mm}$.

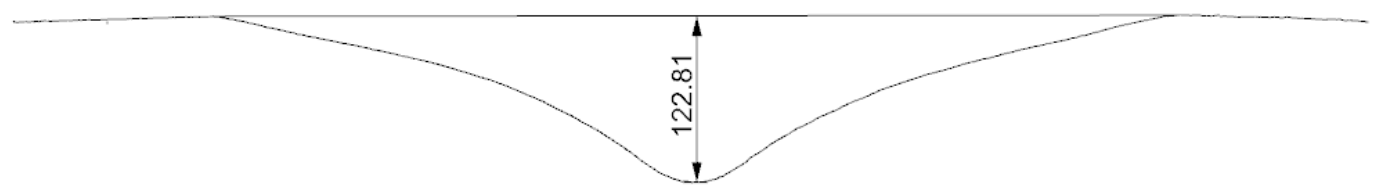

Figure C.3 Post-event deformation profile for Event 3, Plate C1.

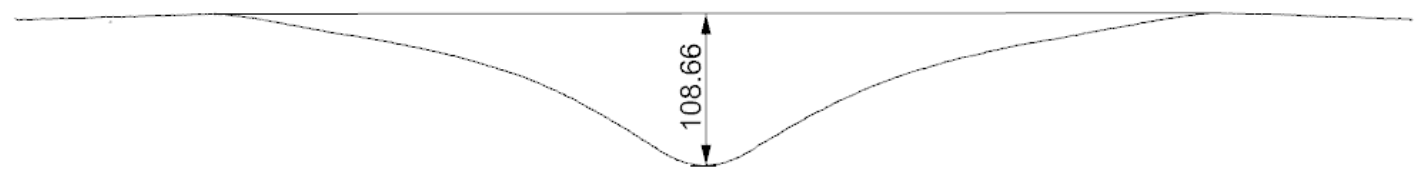

Figure C.4 Post-event deformation profile for Event 4, Plate B2. The dimension is in $\mathrm{mm}$. 
Appendix C. Plate Profiles Measured Using the 3D Laser Scanner.

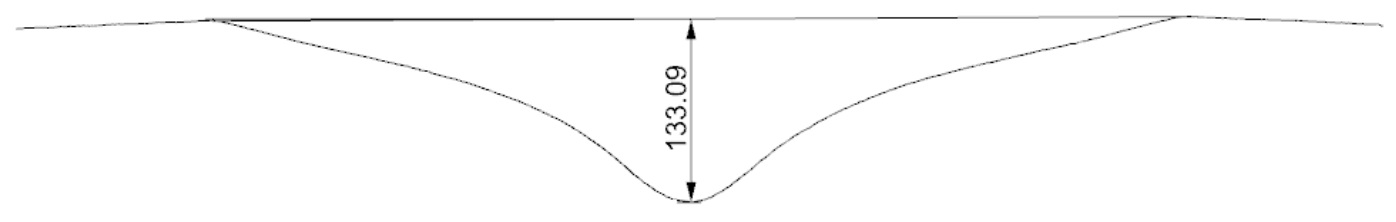

Figure C.5 Post-event deformation profile for Event 5, Plate D2. The dimension is in $\mathrm{mm}$.

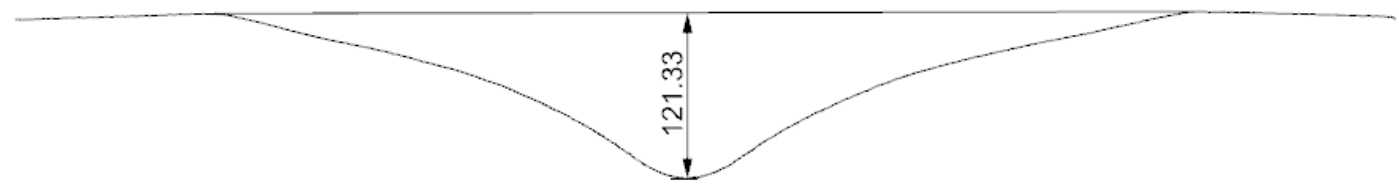

Figure C.6 Post-event deformation profile for Event 6, Plate C2.

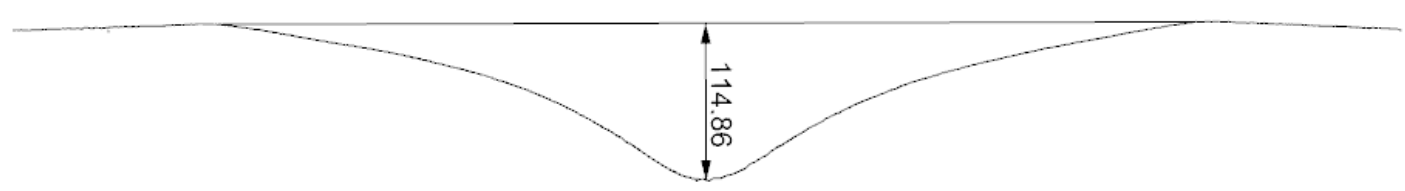

Figure C.7 Post-event deformation profile for Event 8, Plate B4. The dimension is in $\mathrm{mm}$.

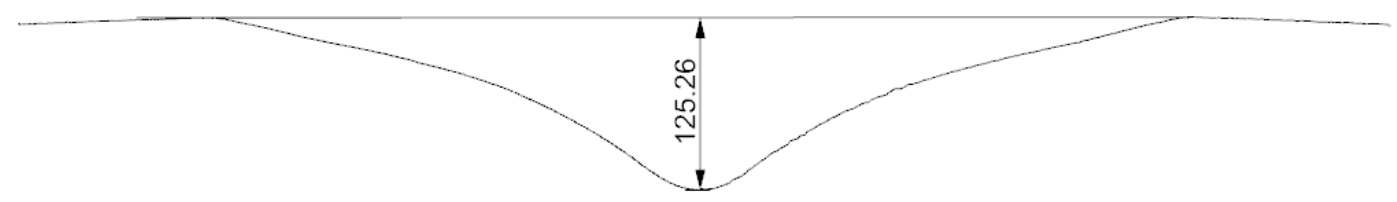

Figure C.8 Post-event deformation profile for Event 9, Plate C3. The dimension is in $\mathrm{mm}$. 


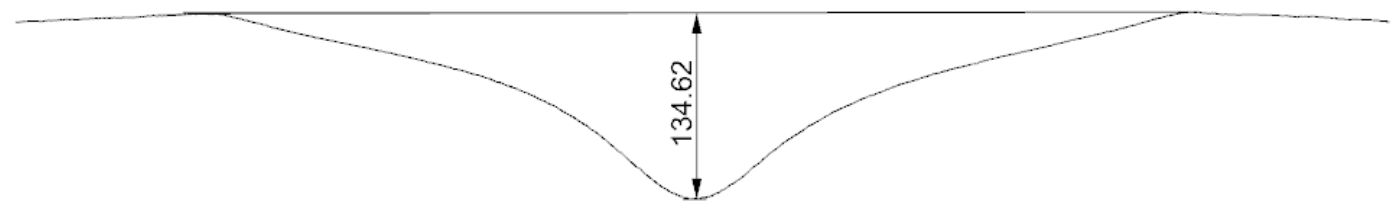

Figure C.9 Post-event deformation profile for Event 10, Plate D3. The dimension is in $\mathrm{mm}$.

Table C.1 Summary of scanned deformation measurements.

\begin{tabular}{llll}
\hline $\begin{array}{l}\text { Experimental plate } \\
\text { deformation }(\mathrm{mm})\end{array}$ & $\begin{array}{l}\text { Scanner measured plate } \\
\text { deformation }(\mathrm{mm})\end{array}$ & Difference $(\mathrm{mm})$ & \% Error \\
\hline 120.4 & 118.9 & 1.5 & 1.21 \\
138.0 & 139.5 & -1.5 & -1.12 \\
125.1 & 122.8 & 2.3 & 1.85 \\
110.0 & 108.7 & 1.3 & 1.17 \\
133.6 & 133.1 & 0.5 & 0.38 \\
120.8 & 121.3 & -0.5 & -0.44 \\
Rupture & N/A & N/A & N/A \\
115.0 & 115.0 & -0.1 & -0.05 \\
126.3 & 125.3 & 1.1 & 0.85 \\
135.7 & 134.6 & 1.0 & 0.76 \\
\hline
\end{tabular}




\section{Appendix D Tensile Test Data}

Test Title: Tensile Testing Of Mild Steel

Test Plan No: FFLTP 944

Test No: FFL 10/19

Customer: K. Ackland

\begin{tabular}{|c|c|c|c|c|c|c|c|c|c|c|c|}
\hline Specimen & $\begin{array}{c}\text { Thickness } \\
(\mathrm{mm})\end{array}$ & $\begin{array}{l}\text { Width } \\
(\mathrm{mm})\end{array}$ & $\begin{array}{l}\text { Area } \\
\text { (Sq. } \\
\mathrm{mm})\end{array}$ & $\begin{array}{c}0.2 \% \\
\text { offset } \\
\text { Load } \\
(\mathrm{kN})\end{array}$ & $\begin{array}{l}0.2 \% \\
\text { offset } \\
\text { Yield } \\
\text { Stress } \\
\text { (Mpa) }\end{array}$ & $\begin{array}{l}\text { Peak } \\
\text { Load } \\
(\mathrm{kN})\end{array}$ & $\begin{array}{l}\text { Peak } \\
\text { Stress } \\
\text { (Mpa) }\end{array}$ & $\begin{array}{l}\text { Initial } \\
\text { Gauge } \\
\text { Length } \\
(\mathrm{mm})\end{array}$ & $\begin{array}{l}\text { Final } \\
\text { Gauge } \\
\text { Length } \\
(\mathrm{mm})\end{array}$ & $\begin{array}{c}\% \\
\text { Elongation }\end{array}$ & Modulus \\
\hline 9-1-L & 3.96 & 12.51 & 49.54 & 17.628 & 355.8 & 24.01 & 484.7 & 50.04 & 66.384 & 32.7 & 182.9 \\
\hline 9-2-L & 3.97 & 12.51 & 49.66 & 18.165 & 365.8 & 24.15 & 486.3 & 49.98 & 61.632 & 23.3 & 183.1 \\
\hline 9-3-L & 3.96 & 12.52 & 49.58 & 17.949 & 362.0 & 23.96 & 484.4 & 49.96 & 67.524 & 35.2 & 217.7 \\
\hline 9-4-L & 3.98 & 12.52 & 49.83 & 17.494 & 351.1 & 23.88 & 479.3 & 50.01 & 67.158 & 34.3 & 182.1 \\
\hline 9-5-L & 3.96 & 12.53 & 49.62 & 17.274 & 348.1 & 23.66 & 476.8 & 50.01 & 65.920 & 31.6 & 246.4 \\
\hline 9-1-T & 3.97 & 12.52 & 49.7 & 19.082 & 383.9 & 24.06 & 484.0 & 50.02 & 64.811 & 29.6 & 200.3 \\
\hline 9-2-T & 3.98 & 12.52 & 49.83 & 18.336 & 368.0 & 24.04 & 482.5 & 50.01 & 67.056 & 34.1 & 187.7 \\
\hline 9-3-T & 3.98 & 12.55 & 49.95 & 18.673 & 373.8 & 24.42 & 488.9 & 50.02 & 66.821 & 33.6 & 152.1 \\
\hline 9-4-T & 3.98 & 12.51 & 49.79 & 18.546 & 372.5 & 24.47 & 491.5 & 50.04 & 65.544 & 31.0 & 197.7 \\
\hline 9-5-T & 3.97 & 12.52 & 49.7 & 18.899 & 380.2 & 24.43 & 491.5 & 50.02 & 64.906 & 29.8 & 195.6 \\
\hline $4 C-1-L$ & 5.92 & 12.52 & 74.12 & 32.527 & 438.8 & 41.75 & 565.3 & 50.06 & 64.148 & 28.1 & 240.0 \\
\hline $4 C-2-\mathrm{L}$ & 5.91 & 12.52 & 73.99 & 32.136 & 434.3 & 41.41 & 559.6 & 49.99 & 65.782 & 31.6 & 208.2 \\
\hline $4 C-3-L$ & 5.91 & 12.52 & 73.99 & 32.509 & 439.3 & 41.69 & 563.4 & 49.98 & 65.082 & 30.2 & 203.4 \\
\hline $4 C-4-L$ & 5.91 & 12.51 & 73.93 & 32.379 & 437.9 & 41.7 & 564.0 & 49.95 & 63.202 & 26.5 & 243.8 \\
\hline 4C-5-L & 5.91 & 12.51 & 73.93 & 32.308 & 437.0 & 41.82 & 565.6 & 49.96 & 63.869 & 27.8 & 211.9 \\
\hline 4C-1-T & 5.92 & 12.51 & 74.06 & 34.647 & 467.8 & 42.96 & 580.1 & 50.01 & 55.550 & 11.1 & 276.5 \\
\hline 4C-2-T & 5.92 & 12.49 & 73.94 & 34.177 & 462.2 & 42.68 & 577.2 & 49.98 & 55.020 & 10.1 & 332.7 \\
\hline 4C-3-T & 5.91 & 12.49 & 73.82 & 32.223 & 436.7 & 41.36 & 560.3 & 49.95 & 61.284 & 22.7 & 341.6 \\
\hline $4 C-4-T$ & 5.89 & 12.48 & 73.51 & 32.35 & 440.1 & 41.27 & 561.4 & 49.98 & 54.873 & 9.8 & 254.2 \\
\hline 4C-5-T & 5.92 & 12.47 & 73.82 & 34.262 & 464.1 & 42.57 & 576.7 & 49.96 & 55.322 & 10.7 & 248.3 \\
\hline
\end{tabular}


Appendix D. Tensile Test Data.

Test Title: Tensile Testing Of Mild Steel

Test Plan No: FFLTP 944

Test No: FFL 10/19

Customer: K. Ackland

\begin{tabular}{|c|c|c|c|c|c|c|c|c|c|c|c|}
\hline Specimen & $\begin{array}{l}\text { Thickness } \\
(\mathrm{mm})\end{array}$ & $\begin{array}{l}\text { Width } \\
(\mathrm{mm})\end{array}$ & $\begin{array}{c}\text { Area } \\
\text { (Sq. } \\
\mathrm{mm})\end{array}$ & $\begin{array}{c}0.2 \% \\
\text { offset } \\
\text { Load } \\
(\mathrm{kN})\end{array}$ & $\begin{array}{l}0.2 \% \\
\text { offset } \\
\text { Yield } \\
\text { Stress } \\
\text { (Mpa) }\end{array}$ & $\begin{array}{l}\text { Peak } \\
\text { Load } \\
(\mathrm{kN})\end{array}$ & $\begin{array}{l}\text { Peak } \\
\text { Stress } \\
\text { (Mpa) }\end{array}$ & $\begin{array}{c}\text { Initial } \\
\text { Gauge } \\
\text { Length } \\
(\mathrm{mm})\end{array}$ & $\begin{array}{l}\text { Final } \\
\text { Gauge } \\
\text { Length } \\
(\mathrm{mm})\end{array}$ & $\begin{array}{c}\% \\
\text { Elongation }\end{array}$ & Modulus \\
\hline No.6-1-L & 4.89 & 12.50 & 61.12 & 23.925 & 391.4 & 30.61 & 500.9 & 49.97 & 59.971 & 20.0 & 208.7 \\
\hline No.6-2-L & 4.87 & 12.50 & 60.88 & 23.721 & 389.7 & 30.86 & 507.0 & 49.98 & 67.802 & 35.7 & 223.3 \\
\hline No.6-3-L & 4.90 & 12.51 & 61.3 & 23.115 & 377.1 & 30.5 & 497.6 & 49.96 & 64.866 & 29.8 & 310.3 \\
\hline No.6-4-L & 4.89 & 12.50 & 61.12 & 23.51 & 384.6 & 30.28 & 495.4 & 49.97 & 65.650 & 31.4 & 178.3 \\
\hline No.6-5-L & 4.89 & 12.50 & 61.12 & 23.508 & 384.6 & 30.47 & 498.5 & 49.99 & 61.920 & 23.9 & 241.4 \\
\hline No.6-1-T & 4.88 & 12.52 & 61.1 & 25.172 & 412.0 & 31.58 & 516.8 & 50.04 & 57.985 & 15.9 & 291.0 \\
\hline No.6-2-T & 4.88 & 12.52 & 61.1 & 25.391 & 415.6 & 31.57 & 516.7 & 49.99 & 61.876 & 23.8 & 206.8 \\
\hline No.6-3-T & 4.89 & 12.52 & 61.22 & 25.377 & 414.5 & 31.52 & 514.8 & 50.01 & 62.030 & 24.0 & 208.4 \\
\hline No.6-4-T & 4.88 & 12.51 & 61.05 & 23.378 & 415.7 & 31.6 & 517.7 & 50.02 & 61.618 & 23.2 & 241.8 \\
\hline No.6-5-T & 4.88 & 12.51 & 61.05 & 25.173 & 412.3 & 31.39 & 514.1 & 50.02 & 56.662 & 13.3 & 279.3 \\
\hline No.7b-1-L & 3.80 & 12.52 & 47.58 & 18.075 & 379.9 & 23.3 & 489.7 & 50.06 & 65.584 & 31.0 & 196.4 \\
\hline No.7b-2-L & 3.83 & 12.51 & 47.91 & 19.476 & 406.5 & 24.25 & 506.2 & 49.92 & 66.028 & 32.3 & 210.6 \\
\hline No.7b-3-L & 3.83 & 12.51 & 47.91 & 19.228 & 401.3 & 24.24 & 506.0 & 50.03 & 66.472 & 32.9 & 216.3 \\
\hline No.7b-4-L & 3.83 & 12.55 & 48.07 & 19.234 & 400.2 & 24.26 & 504.7 & 50.09 & 66.032 & 31.8 & 202.6 \\
\hline No.7b-5-L & 3.79 & 12.50 & 47.38 & 18.037 & 380.7 & 23.12 & 488.0 & 49.98 & 64.673 & 29.4 & 202.0 \\
\hline No.7b-1-T & 3.90 & 12.53 & 48.87 & 19.982 & 408.9 & 25.05 & 512.6 & 50.01 & 62.518 & 25.0 & 221.2 \\
\hline No.7b-2-T & 3.92 & 12.53 & 49.12 & 20.176 & 410.8 & 25.31 & 515.2 & 50.03 & 66.510 & 32.9 & 207.8 \\
\hline No.7b-3-T & 3.91 & 12.52 & 48.95 & 20.187 & 412.4 & 25.35 & 517.9 & 50.02 & 65.632 & 31.2 & 216.5 \\
\hline No.7b-4-T & 3.91 & 12.52 & 48.95 & 20.167 & 412.0 & 25.3 & 516.7 & 50.02 & 66.222 & 32.4 & 216.4 \\
\hline No.7b-5-T & 3.90 & 12.52 & 48.83 & 19.91 & 407.8 & 24.94 & 510.7 & 50.04 & 62.370 & 24.6 & 228.2 \\
\hline
\end{tabular}


Appendix E International Journal of Impact Engineering Paper 


\title{
Deformation of polyurea-coated steel plates under localised blast loading
}

\author{
Kathryn Ackland ${ }^{\mathrm{a}, *}$, Christopher Anderson $^{\mathrm{a}}$, Tuan Duc Ngo ${ }^{\mathrm{b}}$ \\ a Defence Science and Technology Organisation (DSTO), 506 Lorimer St, Fishermans Bend, Victoria 3207, Australia \\ ${ }^{\mathrm{b}}$ Department of Infrastructure Engineering, The University of Melbourne, Victoria 3010, Australia
}

\section{A R T I C L E I N F O}

\section{Article history:}

Received 14 December 2011

Received in revised form 20 July 2012

Accepted 20 August 2012

Available online 11 September 2012

\section{Keywords:}

Polyurea

Blast

Impulsive loading

AUTODYN

Mooney-Rivlin

\begin{abstract}
A B S T R A C T
Experimental and numerical studies were conducted to investigate the effect of polyurea coatings on the blast resistance of mild steel plates. Square steel plates with and without polyurea coatings applied to the back surface were subjected to localised blast loading, both experimentally and numerically using ANSYS $^{\circledR}$ AUTODYN ${ }^{\circledR}$. Two coating thicknesses were considered which were chosen such that each of the plates had the same areal density of $4.7 \mathrm{~g} / \mathrm{cm}^{2}$ over the test area. The residual deformations of the plates were measured after each event and found to increase with coating thickness. The transient deformations of the plates were captured using high speed video. The video revealed that the polyurea coatings de-bonded over a circular area, resulting in a hyperelastic extension of the polyurea and a maximum transient deformation approximately twice that of the bare steel plates. Close agreement was found between the experimental and numerical results for the residual deformations; however, the peak transient deformations were under-predicted in the numerical models. This lead to the development of new Mooney-Rivlin material model constants for the polyurea which gave improved results. It was found that a numerical bond strength of $80 \mathrm{MPa}$ between the polyurea and the steel gave the closest match with the experimental results. Further numerical modelling which varied coating thickness and location found no coating solutions which deformed less than a bare steel plate of equivalent areal density.
\end{abstract}

Crown Copyright $\odot 2012$ Published by Elsevier Ltd. All rights reserved.

\section{Introduction}

A number of studies have explored the use of polyurea coatings to enhance blast protection. In earlier investigations, polyurea was applied to concrete masonry wall units [1-4]. These studies showed that the polymeric material prevents fragments from breaking off the back surface of the wall, effectively holding it together during the blast event. In more recent studies, organisations have considered the use of polymeric materials to enhance the blast protection of metallic structures [5-8] and composite structures [9-12].

The addition of a polyurea coating to an existing metallic structure can be advantageous over a metallic appliqué solution because a polyurea coating can easily be retrofitted to the structure. The work presented in this paper was conducted to investigate whether it would be beneficial to include polymer coatings at the design stage of a blast resistant steel structure, or if it would be more effective to use a thicker metallic solution of equivalent mass.

\footnotetext{
* Corresponding author. Tel.: +61 39626 8566; fax: +61 396268999.

E-mail address: kathryn.ackland@dsto.defence.gov.au (K. Ackland).
}

This was evaluated through experimental testing and numerical modelling.

A simplified quasi-static analysis would lead to the conclusion that polyurea-coated plates will deform more than steel plates of equivalent areal density, due to decreased bending stiffness. However, the behaviour of these materials under blast loading is difficult to predict analytically. High deformation, localised blast loading results in a time-dependent, non-linear scenario where the plates undergo large plastic deformations. Furthermore, both steel and polyurea exhibit strain and strain rate dependent behaviour. For these reasons, experimentally validated numerical simulations are needed to evaluate the comparative performance.

The experiments presented in this paper consider polyurea coatings applied to the back surface of steel plates; however front face coatings are also investigated numerically. Amini et al. [5,6] reported on the effect of the location (front versus back) of polyurea coatings on circular steel plates. They subjected the plates to impulsive loading using a reverse ballistic technique, and found that the polyurea applied to the loading surface of steel plates provided no improvement on the performance of the plate, but rather appeared to promote failure of the plate. The authors explain that this effect is likely due to the compression of the polyurea 


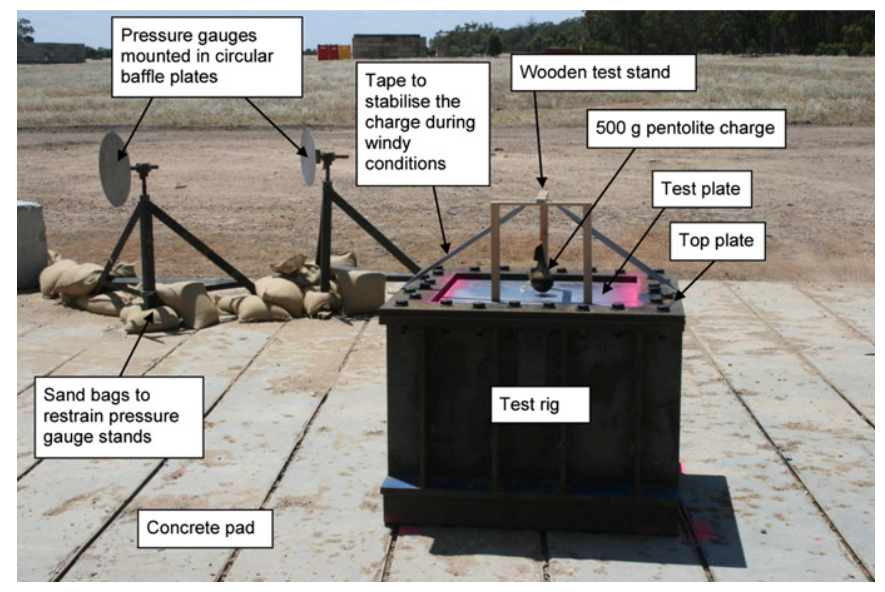

Fig. 1. Experimental setup showing the test plate and charge in position on the test rig.

under the impulsive load, which increases its stiffness, thereby attaining a better impedance match with the steel plate. As a result, more energy is transferred to the plate. However on the opposite surface, part of the initial shock loading on the steel plate was captured and dissipated by the polyurea layer, reducing the deformation and preventing plate fracture. Previous work by the Defence Science and Technology Organisation (DSTO) also showed that polyurea can reduce the residual deformation of plates under blast loading when applied as an appliqué to the back face of mild steel plates [8].

The numerical modelling presented in this paper uses ANSYS ${ }^{\circledR}$ AUTODYN $^{\circledR}$ to model the response of bare and polyurea-coated steel plates subjected to blast loading. Amini et al. [5] numerically simulated impulsive loading of bilayer steel/polyurea plates in LSDYNA and found that a strength of $100 \mathrm{MPa}$ correlated most closely with their experiments. Here we also investigate the effect of bond strength on plate deformation. Numerical models are also used to investigate the effect of coating thickness and location.

\section{Experimental}

Experiments were performed at the Australian Army's Joint Proof and Experimental Unit (JPEU) Graytown, Victoria. Fig. 1 shows the experimental setup which used a blast test rig designed by the Defence Science and Technology Organisation (DSTO) and manufactured by Prototype Engineering Centre (PEC) Laverton. The blast load was generated using nominally $500 \mathrm{~g}$ pentolite spheres, which were centrally detonated using RP501 exploding bridgewire (EBW) detonators. The charges were suspended above the centre of the test plates with a $10 \mathrm{~mm}$ gap between the bottom of the charge and the plate.

\subsection{Test plates}

Test plates were manufactured by DSTO with the assistance of PEC Laverton. Bluescope XLERPLATE 350 grade steel was chosen as the base material due to its widespread availability, with mechanical properties as shown in Table 1 [13]. The plate

Table 1

Bluescope 350 XLERPLATE ${ }^{\circledR}$ mild steel mechanical properties [13].

\begin{tabular}{lll}
\hline & Guaranteed minimum & Typical \\
\hline Yield strength (MPa) & 360 & $410-540$ \\
Tensile strength (MPa) & 450 & $480-590$ \\
\hline
\end{tabular}

dimensions were $1000 \mathrm{~mm} \times 1000 \mathrm{~mm}$ and had bolt holes drilled to match the bolt holes of the blast test rig. Three plate configurations were tested, which were chosen to use readily available thicknesses of steel and had equal areal densities over their test areas:

1. $6 \mathrm{~mm}$ bare steel plates

2. $5 \mathrm{~mm}$ steel plates with a thin coating

3. $4 \mathrm{~mm}$ steel plates with a thick coating

Each configuration was tested three times under the same blast loading conditions to generate a reliable data set, resulting in nine events in total. An areal density of $4.7 \mathrm{~g} / \mathrm{cm}^{2}$ was selected based on a $6 \mathrm{~mm}$ bare steel plate, and polyurea coatings were applied to $4 \mathrm{~mm}$ and $5 \mathrm{~mm}$ plates to give the same areal density over the test section. The coatings were applied on a mass basis rather than thickness to ensure that the areal density of all the plates were the same over the coated area. The thickness was then measured as the average over a number of points along each edge of the polyurea coatings. The average coating thicknesses were $15.7 \mathrm{~mm}$ and $7.7 \mathrm{~mm}$ for the $4 \mathrm{~mm}$ and $5 \mathrm{~mm}$ plates, respectively.
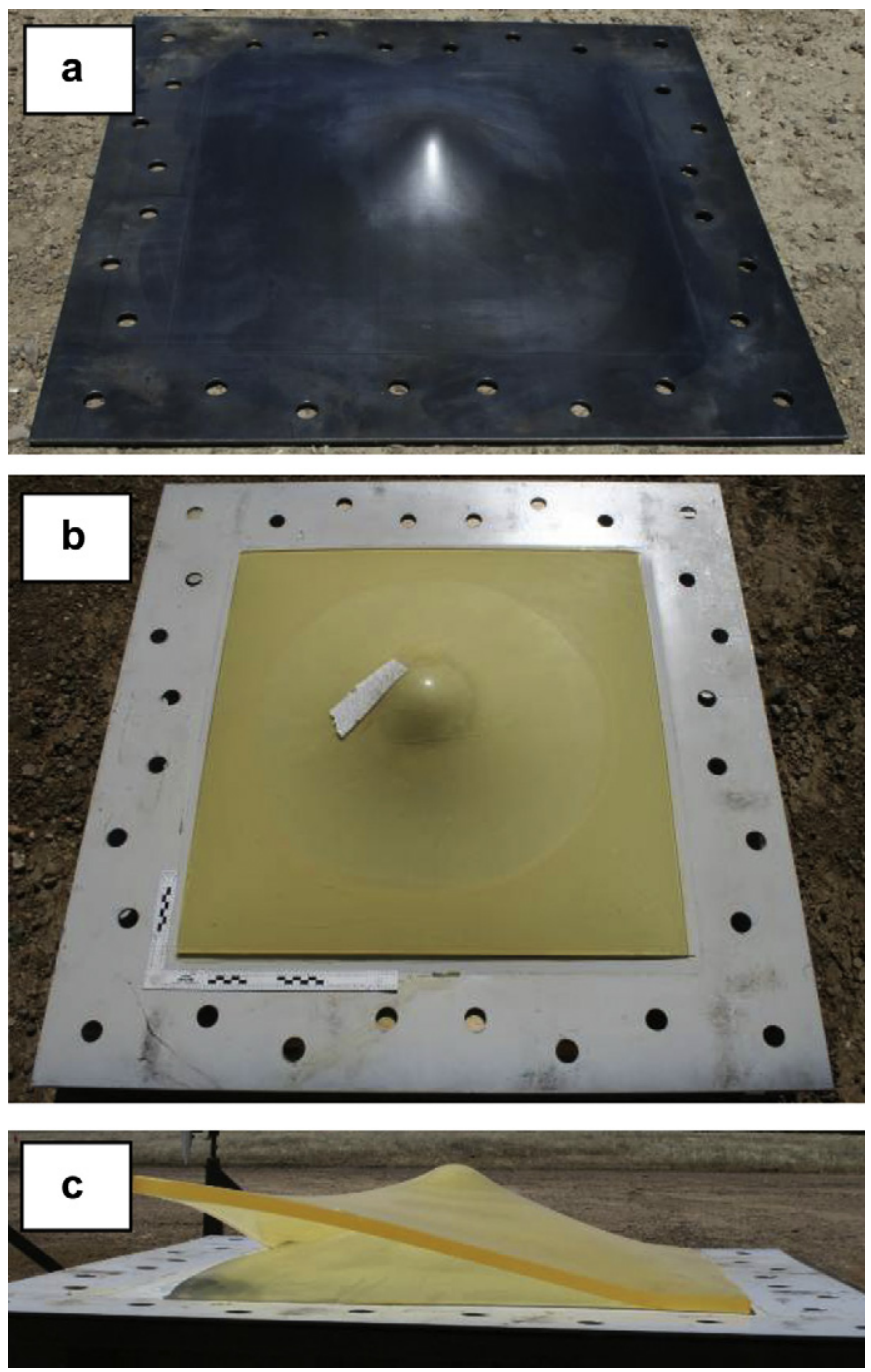

Fig. 2. Deformation of the plates post event: a) Bare steel plate; b) $5 \mathrm{~mm}$ plate with polyurea coating showing de-bonding over the central plate area; c) $4 \mathrm{~mm}$ plate with polyurea coating showing de-bonding reaching the polyurea boundary. 


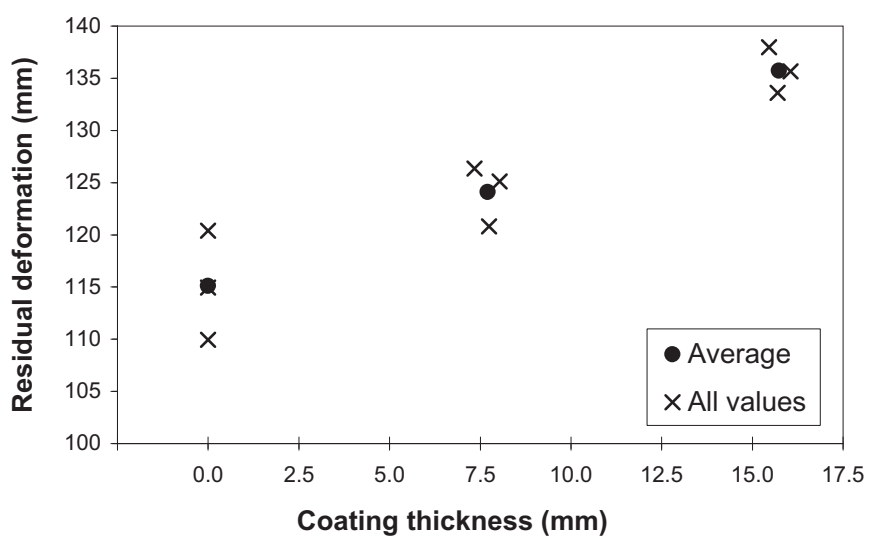

Fig. 3. Experimental results showing the effect of polyurea thickness on the residual deformation of the steel plate.

The plates to which polyurea coatings were applied were first grit blasted and a primer was applied to the plate surface. The polyurea coatings were then cast onto the plates covering an area of $670 \times 670 \mathrm{~mm}$, which is slightly smaller than the test area $(700 \times 700 \mathrm{~mm})$, to allow the coatings to sit inside the test rig. This also ensures that the boundary conditions are similar between the events. The polyurea coatings were made by DSTO using Versalink ${ }^{\circledR}$ $\mathrm{P}-1000$ [14] and Isonate $143 \mathrm{~L}^{\circledR}$ [15]. All coatings were prepared over a week prior to testing and cured in a $40{ }^{\circ} \mathrm{C}$ oven.

\subsection{Instrumentation}

Pressure gauges and high speed video cameras were employed to capture data during the events. Two $\mathrm{PCB}^{\circledR}$ model 101A05 pressure gauges with a range of $689 \mathrm{kPa}(100 \mathrm{psi})$ and a $5.0 \mathrm{MHz}$ sampling rate were used during all nine events to monitor the consistency of the blast waves during the events. The first six events were otherwise un-instrumented to ensure that the plates would not rupture and cause damage within the blast rig under the blast load in subsequent events.

During the third test of each of the three plate configurations, a Phantom Miro-3 high speed camera was mounted within the test rig to record the dynamic deformation of the plate. A resolution of $624 \times 112$ pixels, a framing rate of 8113 frames per second and a shutter speed of $5 \mu$ s were used. A halogen light was used to illuminate the inside of the test rig and to allow enough light to see the image.

\subsection{Experimental results}

Fig. 2 shows the plates after the blast events. The polyureacoated plates showed signs of de-bonding of the polyurea. The diameter of the de-bonded area was measured for each plate using a level ruler positioned across the centre of the plate and plumb bobs to locate the extent of the delamination. The thinner polyurea coatings on the $5 \mathrm{~mm}$ steel plates showed de-bonding over a circular area at the centre of the plate, Fig. $2 b$, with an average diameter of $495 \mathrm{~mm}$ for the de-bonded area. The thicker polyurea coatings on the $4 \mathrm{~mm}$ steel plates all de-bonded across a large area extending to the edges of the plate in all three cases, Fig. 2c. In a practical application, extending the polyurea into the bolted area may be able to prevent complete delamination.

The final deformation of each plate was measured after the event before the test plate was unbolted from the test rig. The deformation was measured as the maximum displacement of the front surface of the plate (the side facing the blast). The 3D plate
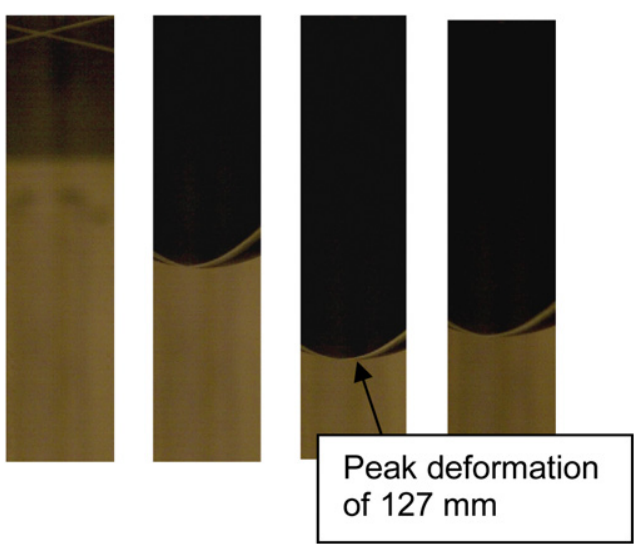

Fig. 4. Still images from the high speed video footage of the $6 \mathrm{~mm}$ bare steel plate. From left to right: pre-detonation, initial plate acceleration, maximum dynamic deformation and final deformation. The centre of the white cross marks the centre of the back surface of the plate.

profiles of the front surfaces of the plates were measured after the plate had been removed from the test rig, using a Konica Minolta Vivid 910 3D laser scanner, to allow comparisons with numerical modelling. The peak deformations measured at the plate centres using the scanner were all within $2 \%$ of the central deformations measured while the plate was still attached to the rig, with the differences believed to be mainly due to relaxation of the plate when it was removed from the rig.

Fig. 3 shows a plot summarising the effect of coating thickness on residual plate deformation. The results for each coating thickness are closely grouped, allowing a clear trend in the data to be seen. It was found that the residual plate deformation increased as the polymer coating thickness was increased.

The high speed videos recorded by the Miro- 3 showed the transient movement of the plates during the blast. Fig. 4 shows selected still images taken from the high speed video footage recorded during Event 7, which tested a bare $6 \mathrm{~mm}$ steel plate. A maximum transient deformation of $127 \mathrm{~mm}$, occurring at the

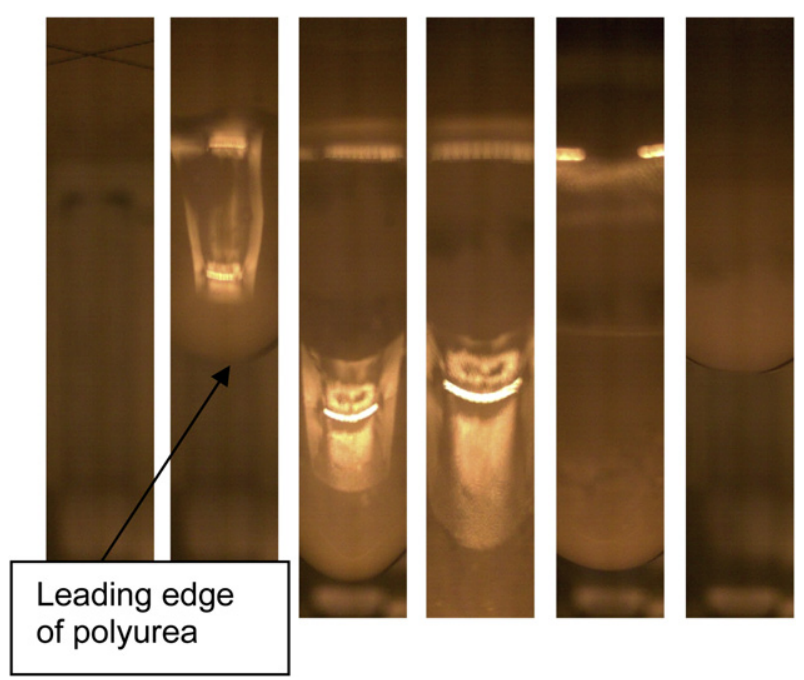

Fig. 5. Still images from the high speed video footage of the $5 \mathrm{~mm}$ plate with a polyurea coating. From left to right: pre-detonation, initial plate acceleration, plate acceleration, polyurea leading edge has moved out of the field of view, deceleration, and final deformation. The centre of the black cross marks the centre of the back surface of the plate. 
centre of the plate was recorded by the high speed camera at $1.78 \mathrm{~ms}$.

Fig. 5 shows a sequence of still images from the high speed video footage recorded during Event 8, which tested a $5 \mathrm{~mm}$ plate with a polyurea coating. The high speed footage shows de-bonding and hyperextension of the polyurea coating during the event, however, it was not possible to determine from the experimental results how early in the event the de-bonding occurred. The maximum extension of the coating was beyond the field of view of the camera. Hence the field of view was adjusted for the following event, resulting in a framing rate of $6908 \mathrm{fps}$. The lighter coloured areas are reflections of the halogen light used to illuminate the test rig interior.

Fig. 6 shows the high speed video footage recorded during Event 9 , which tested a $4 \mathrm{~mm}$ plate with a polyurea coating. This footage also revealed de-bonding and hyperextension of the polyurea coating during the event. The maximum transient deformation of the $15.7 \mathrm{~mm}$ polyurea coating was $266 \mathrm{~mm}$ occurring at $1.11 \mathrm{~ms}$. The maximum transient deformation of the $7.7 \mathrm{~mm}$ polyurea coating on the $5 \mathrm{~mm}$ plate was not recorded, due to the extension of the polymer beyond the field of view of the camera during Event 8 . To estimate the maximum transient deformation of the thin polyurea coating using the available data points, a trend line was fitted to the data. To test the reliability of this method, a similar set of points was removed from the results for the $4 \mathrm{~mm}$ plate with the thick coating and the same type of curve fit was applied. After trialling a number of types of curve fit, a sixth order polynomial gave the best results, with an estimated value of $257 \mathrm{~mm}$ for the peak deformation of the polyurea.

The experiments showed that the peak transient deformations of surface of the polyurea coatings were approximately double that of the peak transient deformations of the bare steel plates. In a practical application, this is expected to be hazardous to objects behind the structure due to the momentum of the polyurea.

The pressure gauges recorded consistent results indicating reliable detonation of the charges for each event.

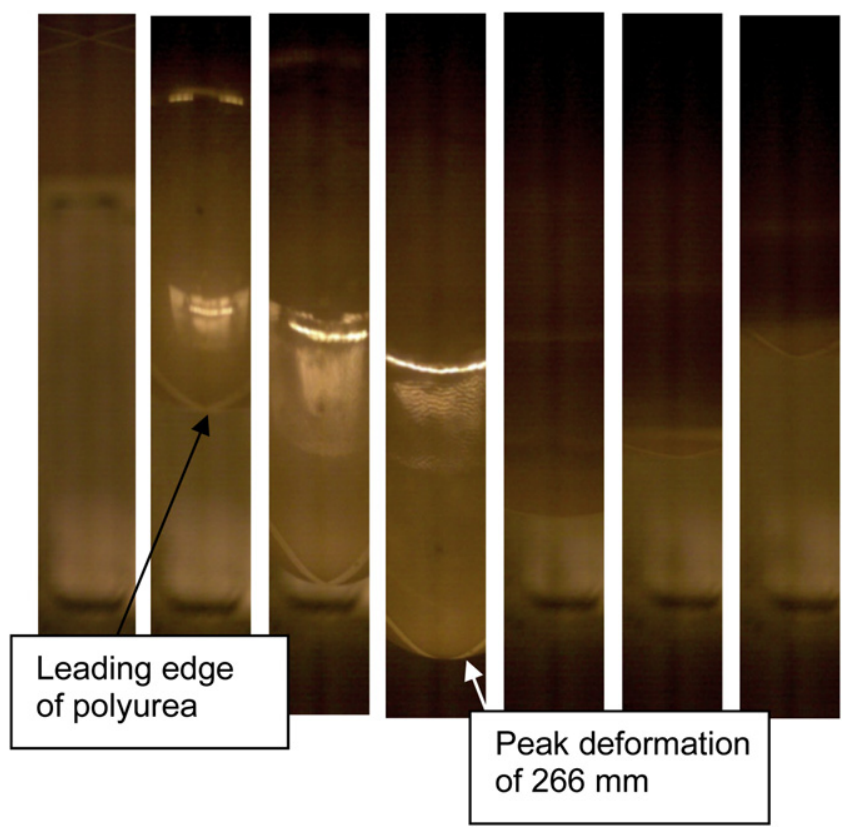

Fig. 6. Still images from the high speed video footage of the $4 \mathrm{~mm}$ plate with a polyurea coating. From left to right the images show the polyurea accelerating, reaching its maximum deformation (fourth image), and returning to its final position. The centre of the white cross marks the centre of the back surface of the plate.

\section{Numerical modelling}

Numerical modelling was conducted using ANSYS ${ }^{\circledR}$ AUTODYN $^{\circledR}$. Fig. 7 shows the model setup for the $6 \mathrm{~mm}$ bare plate. The model uses quarter symmetry in order to speed up the calculation time. The air region was modelled using a 3D Eulerian grid with dimensions of $350 \mathrm{~mm} \times 350 \mathrm{~mm} \times 200 \mathrm{~mm}$ discretised into $1,452,000$ elements $(110 \times 110 \times 120$ elements $)$, with the mesh weighted so there were smaller elements $(1.3 \times 1.3 \times 1.3 \mathrm{~mm})$ near the centre of the plate where the highest pressures were expected. This element size was chosen based on the results of a mesh resolution study. The largest elements located at the boundaries were $13 \mathrm{~mm} \times 13 \mathrm{~mm} \times 8 \mathrm{~mm}$. The $X=0$ and $Y=0$ planes were set as symmetry planes, and a "flow out" condition was applied to the remaining four surfaces of the grid.

The steel plate was modelled using four-noded quadrilateral shell elements of $6 \mathrm{~mm}$ thickness and a weighted mesh such that there were smaller elements near the plate centre. The lateral dimensions of the elements at the plate centre were $1.8 \mathrm{~mm} \times 1.8 \mathrm{~mm}$ and the elements at the boundary were $5 \mathrm{~mm} \times 5 \mathrm{~mm}$. The dimensions of the plate were $350 \mathrm{~mm} \times 350 \mathrm{~mm}$ and the plate was located in the $Z=0$ plane. The non-symmetry boundaries of the plate were fixed to represent the clamped edges by setting all translational and rotational velocities to zero at the nodes. A gauge was inserted at the origin to record the displacement at the plate centre.

The plates with polyurea coatings were modelled by adding a Lagrangian part to represent the polyurea. The polyurea was modelled using 8-node hex elements with the nodes of the polyurea mesh aligned with the nodes of the plate along the polyurea/ plate interface, and covering an area of $335 \times 335 \mathrm{~mm}$. The $7.7 \mathrm{~mm}$ and $15.7 \mathrm{~mm}$ coatings had 4 and 8 elements through their thicknesses, respectively.

To simulate polyurea de-bonding in the numerical models, a breakable bond was modelled between the polyurea and the plate. This was achieved by adding a thin layer of elements between the two materials. The layer had a single element through its thickness of $0.2 \mathrm{~mm}$.

\subsection{Material models}

The material model used for the Bluescope XLERPLATE 350 steel was a modified version of a material available from the AUTODYN material library. The material "Steel 4340 " was used as a template and modified in such a way that the Johnson-Cook strength model [16] had the same parameters as Balden and Nurick [17] (who also

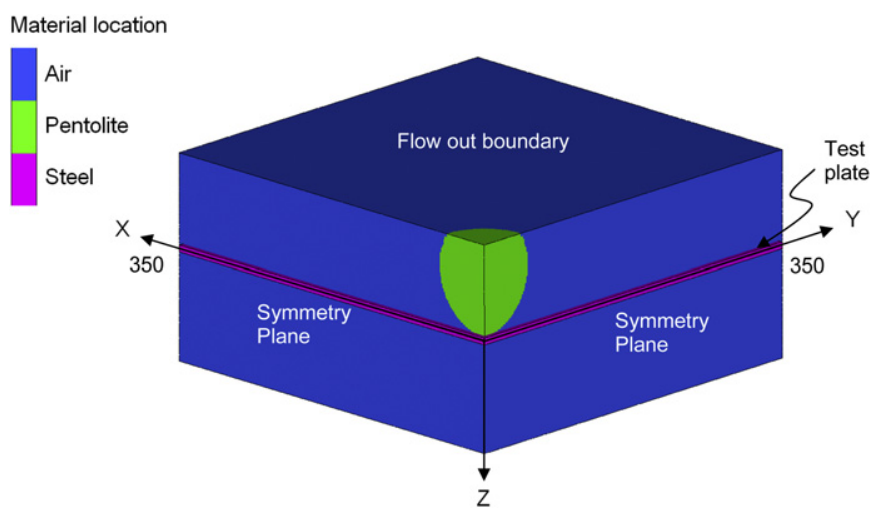

Fig. 7. Model setup for air blast on a steel plate in AUTODYN. 
modelled mild steel). The Johnson-Cook strength model describes the material flow stress using the following equation [16]:

$\bar{\sigma}=\left[\sigma_{0}+B \bar{\varepsilon}_{\mathrm{p}}^{\mathrm{n}}\right]\left[1+C \log \left(\frac{\dot{\bar{\varepsilon}}_{p}}{\dot{\varepsilon}_{0}}\right)\right]\left[1-\widehat{\theta}^{\mathrm{m}}\right]$

where

$\widehat{\theta}=\left[\left(\frac{T-T_{\mathrm{r}}}{T_{\text {melt }}-T_{\mathrm{r}}}\right)\right]$

$\bar{\sigma}=$ yield stress at non-zero strain rate $(\mathrm{Pa}), \bar{\varepsilon}_{\mathrm{p}}=$ equivalent plastic strain $\dot{\bar{\varepsilon}}_{\mathrm{p}}=$ normalized equivalent plastic strain rate $\left(\mathrm{s}^{-1}\right)$, $\dot{\varepsilon}_{0}=$ reference strain rate $\left(\mathrm{s}^{-1}\right), T=$ material temperature $(\mathrm{K})$ $T_{\mathrm{r}}=$ room temperature $(\mathrm{K})$ and $T_{\text {melt }}=$ material melting temperature (K). $B, n, C$ and $m$ are material dependent parameters that may be determined empirically. The yield strength of the steel, $\sigma_{0}$, was adjusted for each plate thickness to match the results of tensile tests of the plate materials. The Johnson-Cook strength model parameters used for each plate thickness are shown in Table 2.

The material models for air and pentolite were taken from the AUTODYN material library [18], using an ideal gas equation of state and a Jones-Wilkinson-Lee (JWL) equation of state, respectively. The material model for the polyurea was developed by the Naval Surface Warfare Centre, Carderock Division (NSWC-CD) using a Mooney-Rivlin hyperelastic material model with constants fitted to data from published work by Roland et al. [19] and Amirkhizi et al. [20], at a strain rate above $300 \mathrm{~s}^{-1}$. The fitted constants were provided by Ken Nahshon, NSWC-CD and incorporated into AUTODYN with the assistance of LEAP Australia. According to the AUTODYN user manual [18], the strain energy function, $\psi$, of a hyperelastic material can be expressed as an infinite series, which, when reduced to the 2-parameter Mooney-Rivlin model is given by:

$\psi=C_{10}\left(\bar{I}_{1}-3\right)+C_{01}\left(\bar{I}_{2}-3\right)+\frac{1}{d}(J-1)^{2}$

where $C_{10}$ and $C_{01}$ are empirically determined material constants, $\bar{I}_{1}$ and $\bar{I}_{2}$ are the first and the second invariant of the deviatoric component of the left Cauchy-Green deformation tensor, $J$ is the determinant of the elastic deformation gradient, and $d$ is the material incompressibility parameter. The parameters used in this study were $C_{10}=875.2 \mathrm{kPa}, C_{01}=6321.3 \mathrm{kPa}$ and $d=4 \times 10^{-7} \mathrm{kPa}^{-1}$.

The bond material was given the same material properties as the polyurea, but with a principle stress failure model added. This

Table 2

Johnson-Cook strength model parameters used to model Bluescope XLERPLATE 350 steel.

\begin{tabular}{ll}
\hline Material model parameter & Value \\
\hline Bulk modulus & $159 \mathrm{GPa}$ \\
Shear modulus & $77 \mathrm{GPa}$ \\
Yield stress, $\sigma_{0}$ & $446 \mathrm{MPa}(6 \mathrm{~mm}$ plate $)$ \\
& $400 \mathrm{MPa}(5 \mathrm{~mm}$ plate $)$ \\
& $402 \mathrm{MPa}(4 \mathrm{~mm}$ plate $)$ \\
Hardening constant, $B$ & $570 \mathrm{MPa}$ \\
Hardening exponent, $n$ & 0.36 \\
Strain rate constant, $C$ & 0.35 \\
Thermal softening exponent, $m$ & 0.55 \\
Melting temperature, $T_{\text {melt }}$ & $1793 \mathrm{~K}$ \\
Room temperature, $T_{\mathrm{r}}$ & $300 \mathrm{~K}$ \\
Ref. strain rate $(/ \mathrm{s}), \dot{\varepsilon}_{0}$ & 1 \\
\hline
\end{tabular}

means that when the bond material reaches the specified failure stress, the material will fail and its strength model will no longer apply. After failure, the bond elements are able to undergo large deformations without affecting the simulation as they no longer carry any stress. The failure stress was determined by adjusting the failure stress in the simulations until the de-bonded area matched the experiments.

\subsection{Euler-Lagrange coupling}

The Euler-Lagrange interaction in AUTODYN was set to fully coupled, allowing the air blast and plate to interact. The bare plate was given an artificial thickness of $6 \mathrm{~mm}$, while artificial thicknesses of $5 \mathrm{~mm}$ and $4 \mathrm{~mm}$ were applied to the $5 \mathrm{~mm}$ and $4 \mathrm{~mm}$ coated plates, respectively. The application of an artificial thickness allows the air to interact with the plate without passing through the plate itself.

\subsection{Simulation in AUTODYN}

The explosive was first simulated using a $60 \mathrm{~mm}$ 1D spherically symmetric Euler model with $0.5 \mathrm{~mm}$ elements. This was achieved by modelling a wedge in AUTODYN, as shown in Fig. 8. The wedge was filled with the material model for pentolite to a radius of $41.5 \mathrm{~mm}$, to match the experimental charge geometry. In the experiment, the detonation is symmetric until the shock interacts with the plate, which occurs at a radius of $51.5 \mathrm{~mm}$. Thus the 1D model was run until the radius of the shock wave was $50 \mathrm{~mm}$. At this point, the $1 \mathrm{D}$ model was remapped onto the $3 \mathrm{D}$ grid with the charge centre positioned $51.5 \mathrm{~mm}$ above the plate, to represent the standoff used in the experiments (Fig. 7). This method of simulating the explosive loading had previously been validated against pressure gauge readings from a previous trial.

The simulations were run until the time reached $0.5 \mathrm{~ms}$, at which point the air was removed from the model. After this time the blast loading of the plate had completed, and the removal of the air grid sped up the calculation time significantly. After $5 \mathrm{~ms}$ a static damping constant was applied to the model to allow the dynamic solution to converge to a state of stress equilibrium.

\subsection{Numerical results}

The strength of the bond between the polyurea and the steel was determined through a series of models of the $5 \mathrm{~mm}$ plate with the $7.7 \mathrm{~mm}$ coating, in which the failure strength of the bond was adjusted until the de-bonded area matched that of the experiment. This resulted in a failure stress of $90 \mathrm{MPa}$. This failure stress was then applied to a simulation using the $15.7 \mathrm{~mm}$ thick coating. Figs. 9 and 10 show screen captures from the simulations of the plates with $7.7 \mathrm{~mm}$ and $15.7 \mathrm{~mm}$ thick polyureas, respectively.

AIR

\section{PENTOLITE}

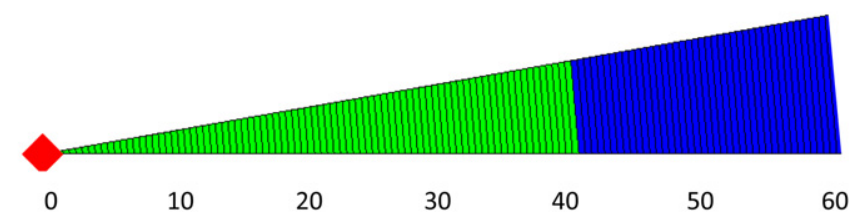

Fig. 8. 1D wedge setup in AUTODYN. The red diamond shows the detonation point. [For interpretation of color referred in this figure legend, the reader is referred to web version of the article.] 

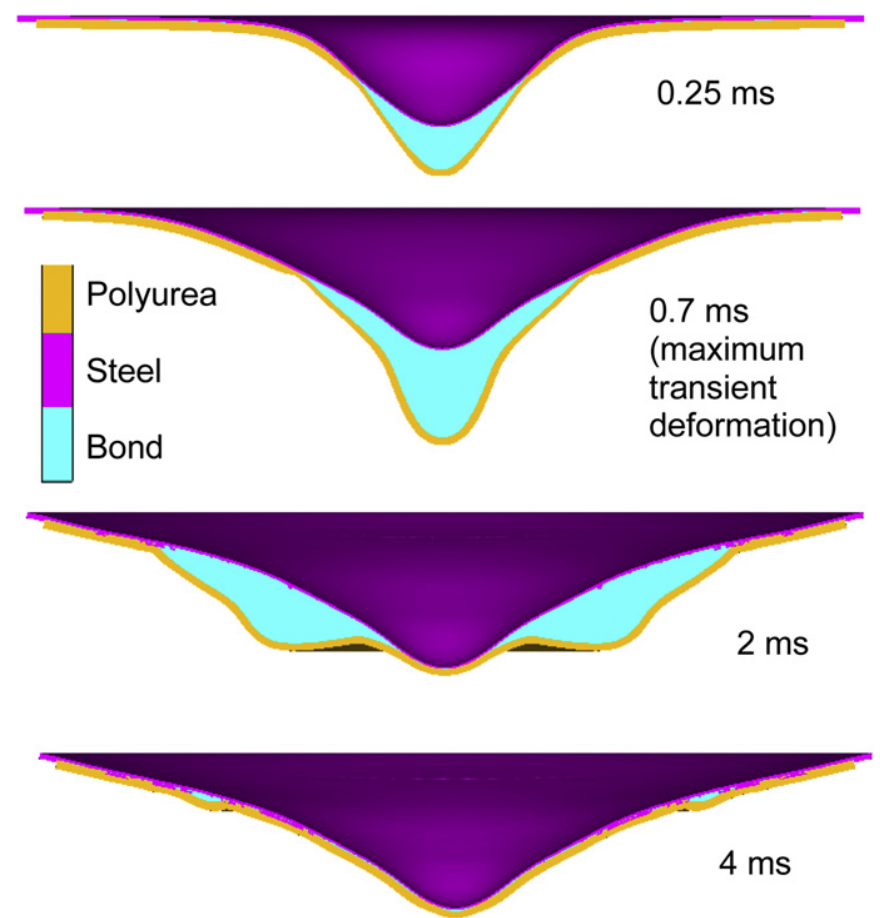

Fig. 9. AUTODYN screen captures showing polyurea de-bonding at selected times from $0.25 \mathrm{~ms}$ to $4 \mathrm{~ms}$ for the $5 \mathrm{~mm}$ steel plate with a $7.7 \mathrm{~mm}$ polyurea coating. These images show half the plate, which has been sectioned across its centre.

The numerical models allowed us to predict when the debonding occurred during the experiment. Initial de-bonding for the plate with the $7.7 \mathrm{~mm}$ polyurea coating was seen at the plate centre at $12 \mu \mathrm{s}$ after detonation. By the time the loading was $95 \%$ complete, de-bonding had occurred to a radius of $81 \mathrm{~mm}$, which is
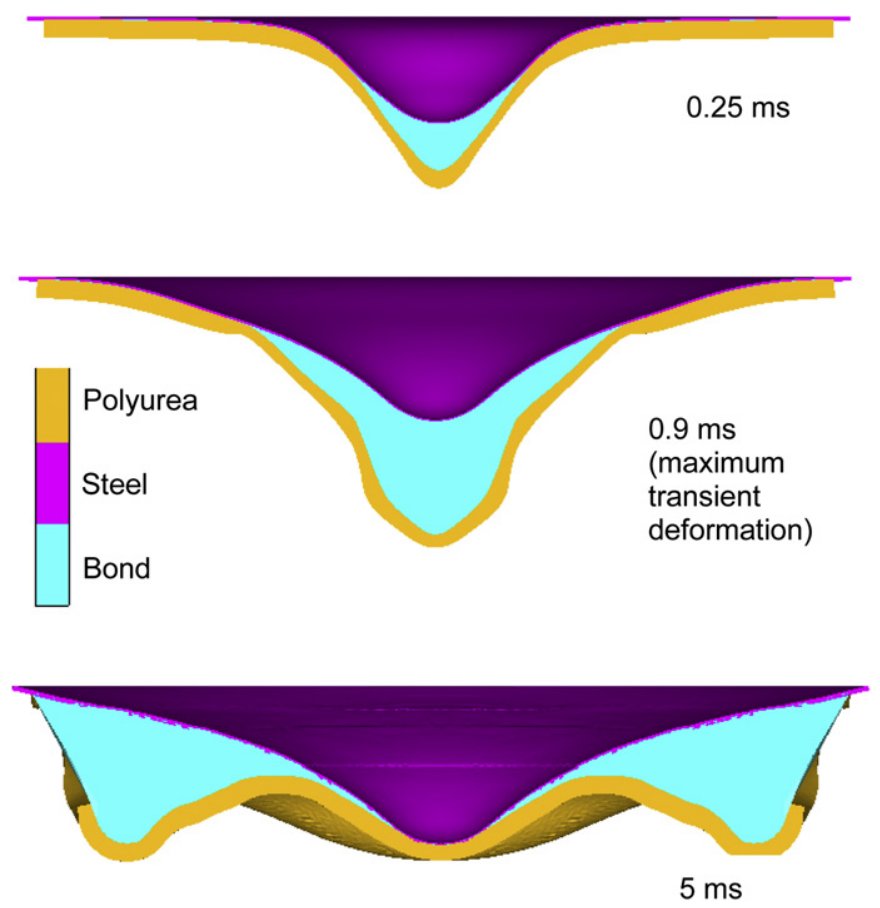

Fig. 10. AUTODYN screen captures showing polyurea de-bonding at selected times from $0.25 \mathrm{~ms}$ to $5 \mathrm{~ms}$ for the $4 \mathrm{~mm}$ steel plate with a $15.7 \mathrm{~mm}$ polyurea coating. These images show half the plate, which has been sectioned across its centre. close to one third of the final de-bonded radius. This means that in terms of area, approximately $90 \%$ of the de-bonding occurred after the loading phase was complete. For the $15.7 \mathrm{~mm}$ coating, similar results were seen, with over $90 \%$ of the de-bonding occurring after the loading phase. This result confirms that the polyurea coatings will have contributed to the plates' responses to the blast loading.

Fig. 11 shows comparisons of the numerical and experimental results for the final deformed plate profiles of the $6 \mathrm{~mm}$ bare plate, the $5 \mathrm{~mm}$ plate with a polyurea coating and the $4 \mathrm{~mm}$ plate with a polyurea coating, respectively. In all three cases the final deformation profile as determined through numerical modelling matches closely with the experimentally measured profile.

Fig. 12 shows a comparison between the numerical and experimental results for the transient deformations of the three plate configurations, where the experimental measurements were obtained from the high speed camera footage. It can be seen that the numerical results under-predict the peak dynamic deformations of the polyurea coatings. The peak deformation of the polyurea in the numerical model for the $5 \mathrm{~mm}$ plate with the thin coating was $197 \mathrm{~mm}$, compared with $257 \mathrm{~mm}$ calculated from the high speed video footage. Also, in the numerical model, the polyurea begins moving back towards the plate at an earlier time $(0.7 \mathrm{~ms}$ compared
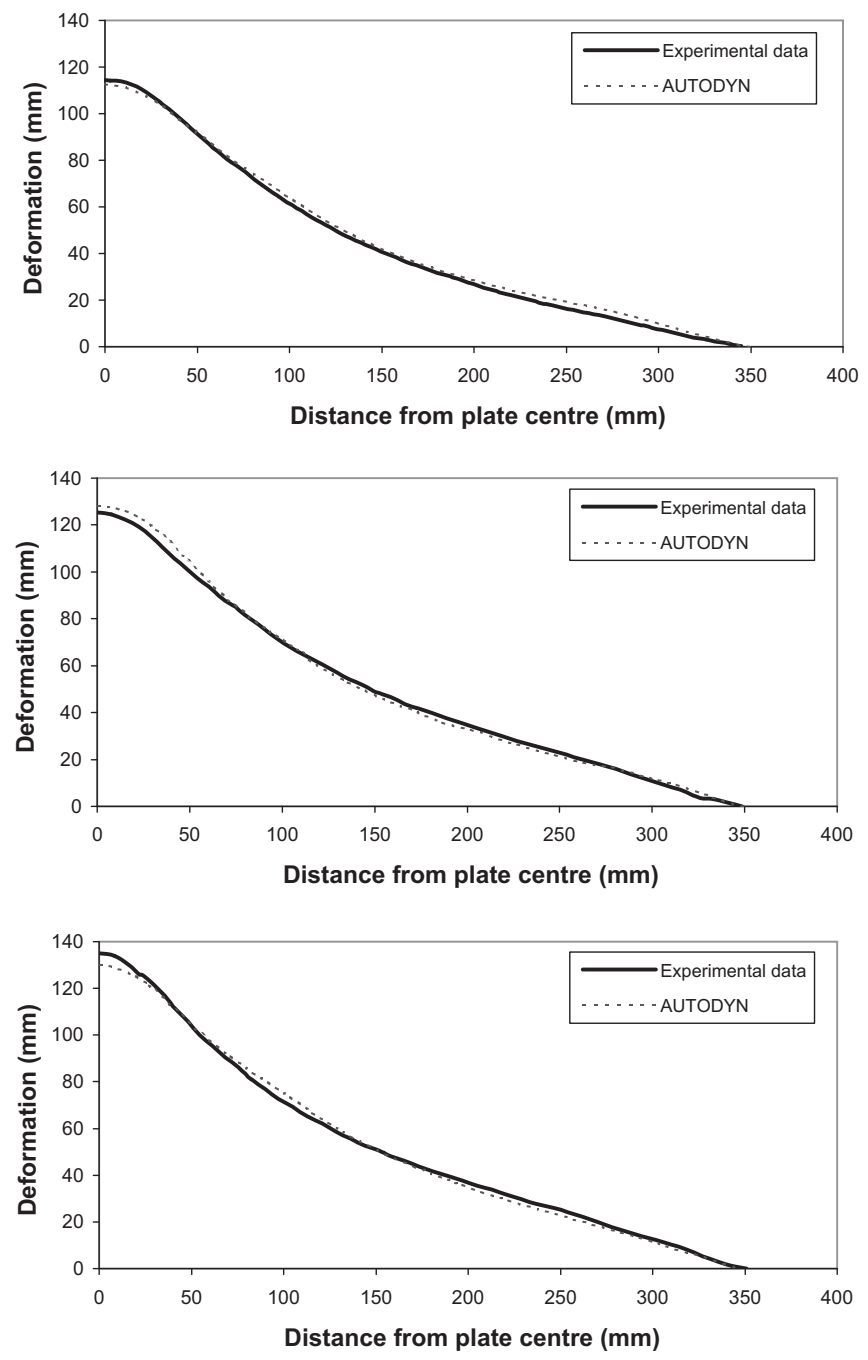

Fig. 11. Comparison of the experimental plate profiles and numerical modelling results. From top to bottom: bare plate, $5 \mathrm{~mm}$ plate with $7.7 \mathrm{~mm}$ polyurea coating, $4 \mathrm{~mm}$ plate with $15.7 \mathrm{~mm}$ polyurea coating. 


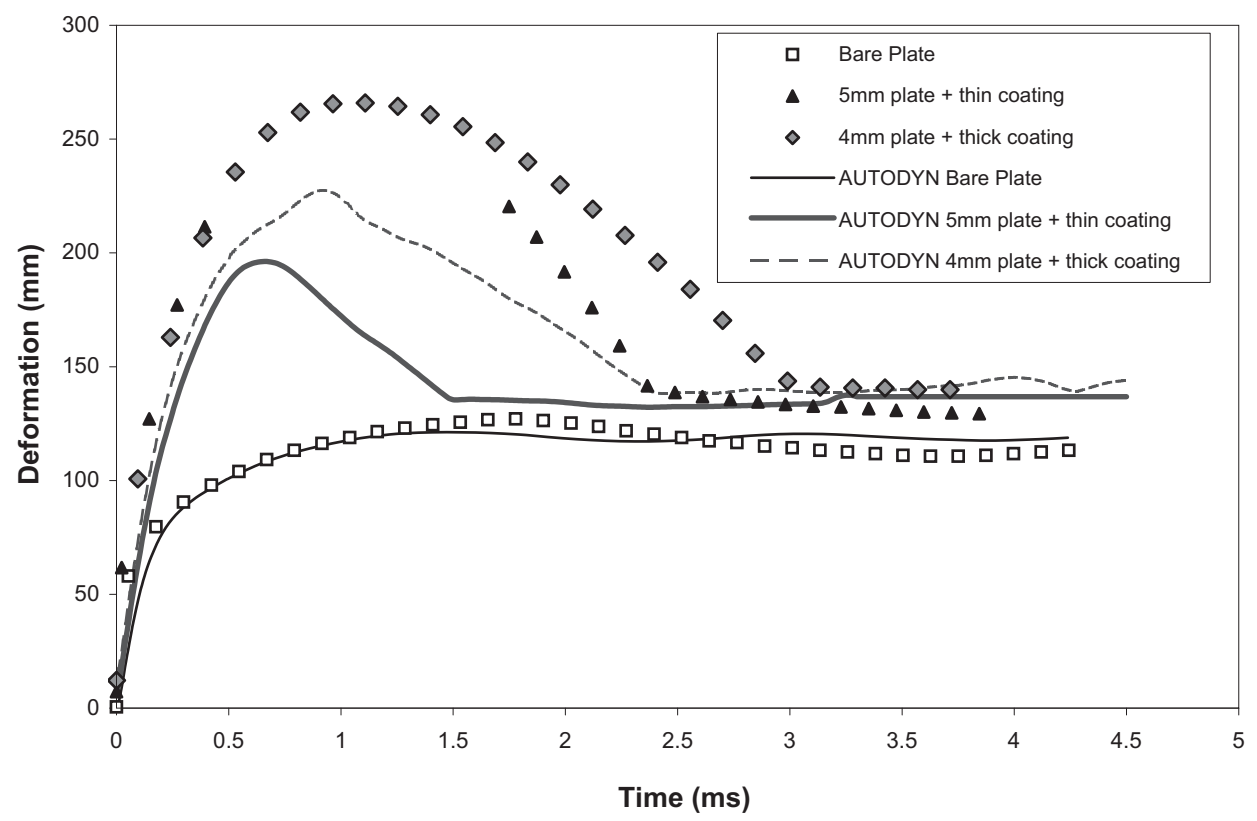

Fig. 12. Comparison of experimental and numerical results for the transient deformation of the plate centre.

with $1 \mathrm{~ms}$ ) and returns to rest against the plate at an earlier time than seen in the experiment ( $1.5 \mathrm{~ms}$ compared with $2.3 \mathrm{~ms})$. The same can be seen for the $4 \mathrm{~mm}$ plate with the thick coating, which reaches a maximum dynamic deformation of $227 \mathrm{~mm}$ in the numerical model compared with $266 \mathrm{~mm}$ as calculated from the experimental high speed video footage. The thick coating in the numerical model also comes back to rest against the plate at an earlier time of $2.3 \mathrm{~ms}$ compared with $3 \mathrm{~ms}$ in the experiment.

The under-predictions for the peak polyurea deformations may be due to the Mooney-Rivlin constants being fit to a strain rate over $300 \mathrm{~s}^{-1}$. This is much higher than the strains seen in the numerical models, which were in the order of $10 \mathrm{~s}^{-1}$. A number of studies have shown that polyurea exhibits strain rate dependent behaviour $[19,21,22]$, which suggests that the constants used here may not be suitable for the application being numerically modelled. To investigate this further, high strain rate tensile testing of the polyurea used in these experiments was conducted. New values for the Mooney-Rivlin constants $C_{10}$ and $C_{10}$ were fit to the data at a strain rate of $10 \mathrm{~s}^{-1}$. Fig. 13 shows the tensile test data measured at various strain rates, along with the fitted data at $10 \mathrm{~s}^{-1}$, using constants $C_{10}=43.9 \mathrm{kPa}, C_{01}=5568.1 \mathrm{kPa}$.

The simulations reported previously were re-run using the new constants. The third Mooney-Rivlin parameter, $d$, was kept as $4 \times 10^{-7} \mathrm{kPa}^{-1}$. The material model for the bond was modified to use the updated Mooney-Rivlin constants, and the bond strength was changed to $80 \mathrm{MPa}$ so that the numerically simulated delaminated area matched that of the experiment.

Fig. 14 shows the residual plate deformations found using the new material model, compared with the experimental results. It was found that although the final deformed shapes match well, the peak was slightly over-predicted for the $5 \mathrm{~mm}$ coated plate and slightly under-predicted for the $4 \mathrm{~mm}$ plate coated plate.

Fig. 15 shows the transient deformations found using the new material model, compared with the experimental results. These results showed an improvement compared to the previous numerical results. The transient deformations for the $4 \mathrm{~mm}$ plate with the thick coating match closely with the experimental data when the new constants are used. The results for the $5 \mathrm{~mm}$ plate with the thick coating do not match the experimental data as well; however, the result is improved compared to the fit seen previously in Fig. 12.

The models developed here were used to investigate the effect of bonding, coating thickness and coating location on the residual plate deformation, which are discussed in the following sections. As only residual deformations were considered, the original MooneyRivlin material model was used, as this model provided more accurate results for residual deformations.

\section{Effect of bonding on residual plate deformation}

The effect of the bond between the plate and the polyurea on the residual plate deformation was investigated using the numerical models which have been developed. The two coating thicknesses were modelled under three conditions: a) with no bond between the plate and the polymer, b) with an unbreakable bond between

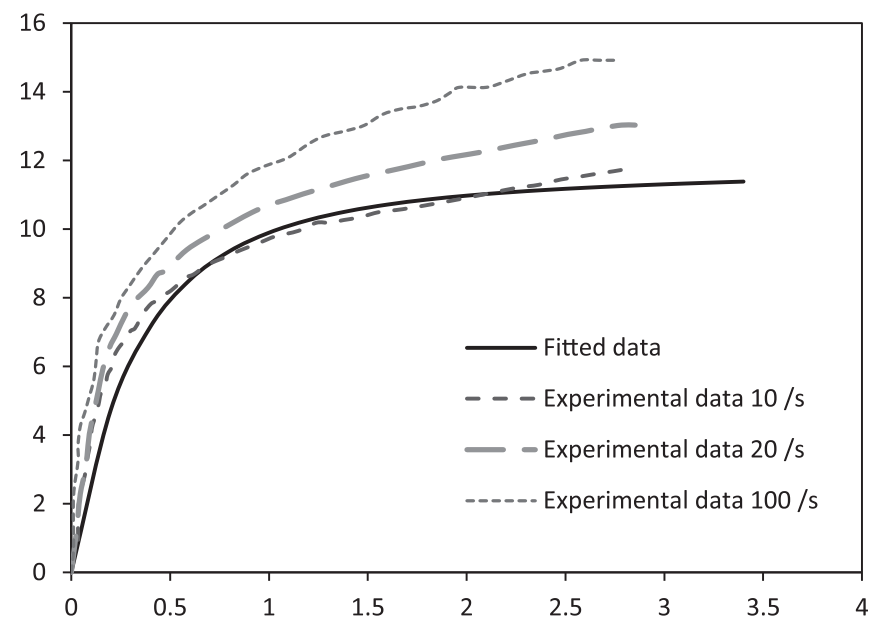

Fig. 13. Results of high strain rate tests on polyurea and fitted data at $10 \mathrm{~s}^{-1}$ using a Mooney-Rivlin hyperelastic material model with $C_{10}=43.9 \mathrm{kPa}$ and $C_{01}=5568.1 \mathrm{kPa}$. 

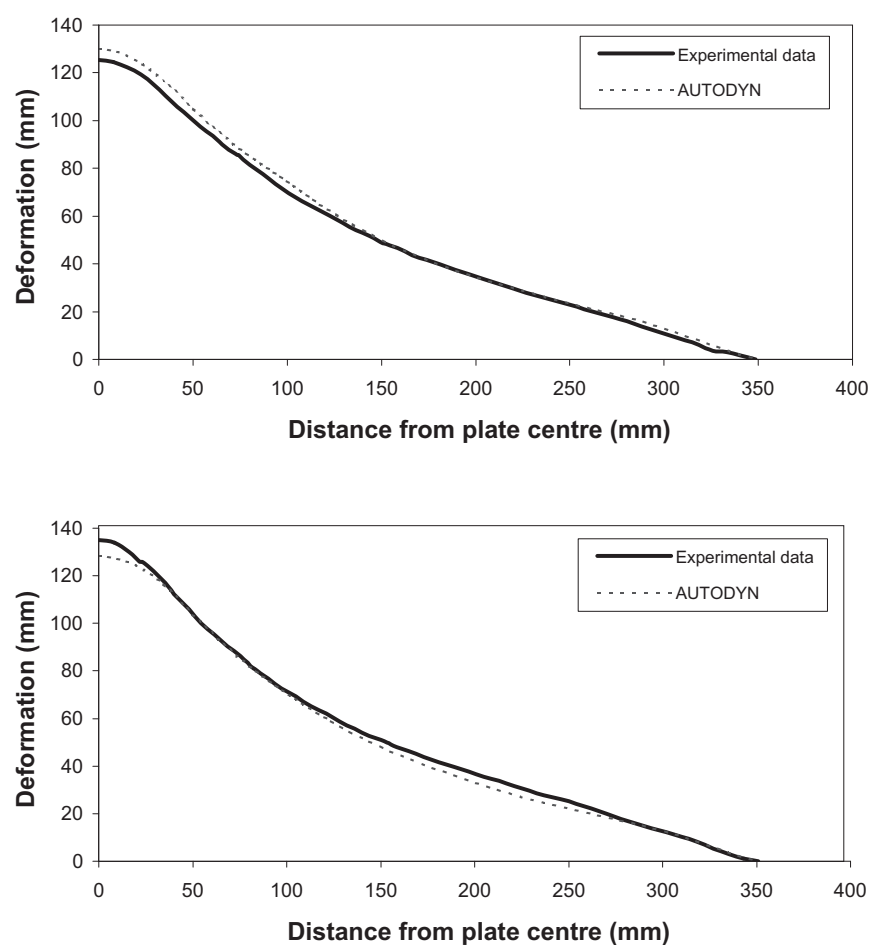

Fig. 14. Comparison of the experimental plate profiles and numerical modelling results using the updated Mooney-Rivlin constants. Top: bare plate, $5 \mathrm{~mm}$ plate with $7.7 \mathrm{~mm}$ polyurea coating. Bottom: $4 \mathrm{~mm}$ plate with $15.7 \mathrm{~mm}$ polyurea coating.

the plate and the polymer and c) with a bond with a failure stress of $90 \mathrm{MPa}$.

To model the coating without a bond, the thin layer of elements representing the bond between the plate and the polyurea was removed. This meant that the plate and polyurea were no longer joined. However, a Lagrange/Lagrange interaction was defined in AUTODYN using a $0.2 \mathrm{~mm}$ interaction gap, so that once the plates began to deform, they were acted upon by the polyurea. To simulate

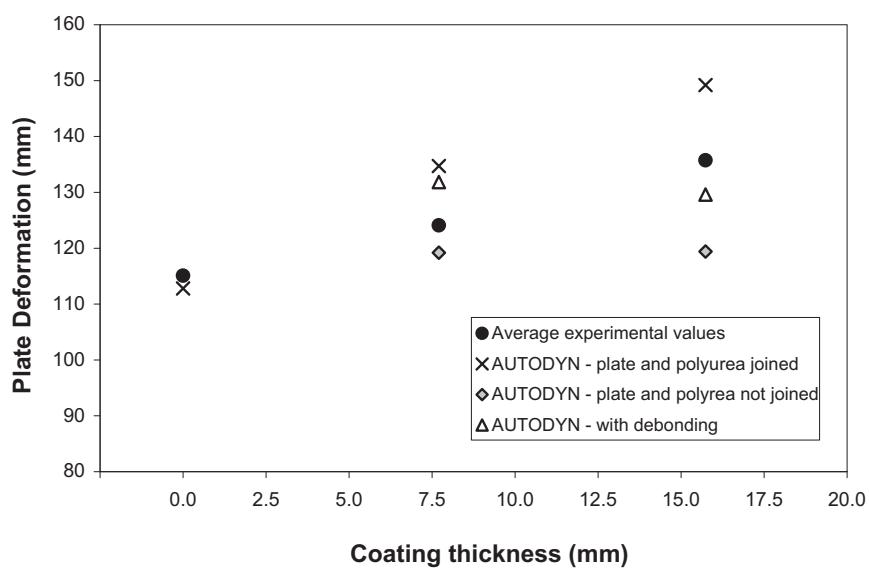

Fig. 16. Effect of modelling polyurea de-bonding compared with experimental results and fully joined and unjoined plate models.

the unbreakable bond, the thin bond layer was removed from the simulation, and the polyurea was joined directly to the plate.

Fig. 16 shows the results of the models with and without joins between the plate and the polyurea, compared with previously described models in which the polyurea de-bonded from the plate, as well as experimental results. The fully joined models produced residual deformations which were higher than the experimental results, and the deformation was seen to increase with coating thickness. The models which did not use joins between the plate and polyurea resulted in lower residual deformations than the experimental results. The results of the unjoined models did not show an increase in deformation with coating thickness, instead the same deformation was found for the two coating thicknesses. The results for the numerical models which simulated the polyurea de-bonding lay between the results for the fully joined and unjoined plates. Although the numerical results with de-bonding were fairly close to the experimental results, with discrepancies of $6.2 \%$ for the $7.7 \mathrm{~mm}$ polyurea coating and $4.8 \%$ for the $15.7 \mathrm{~mm}$ polyurea coating, the simulations did not show the trend of

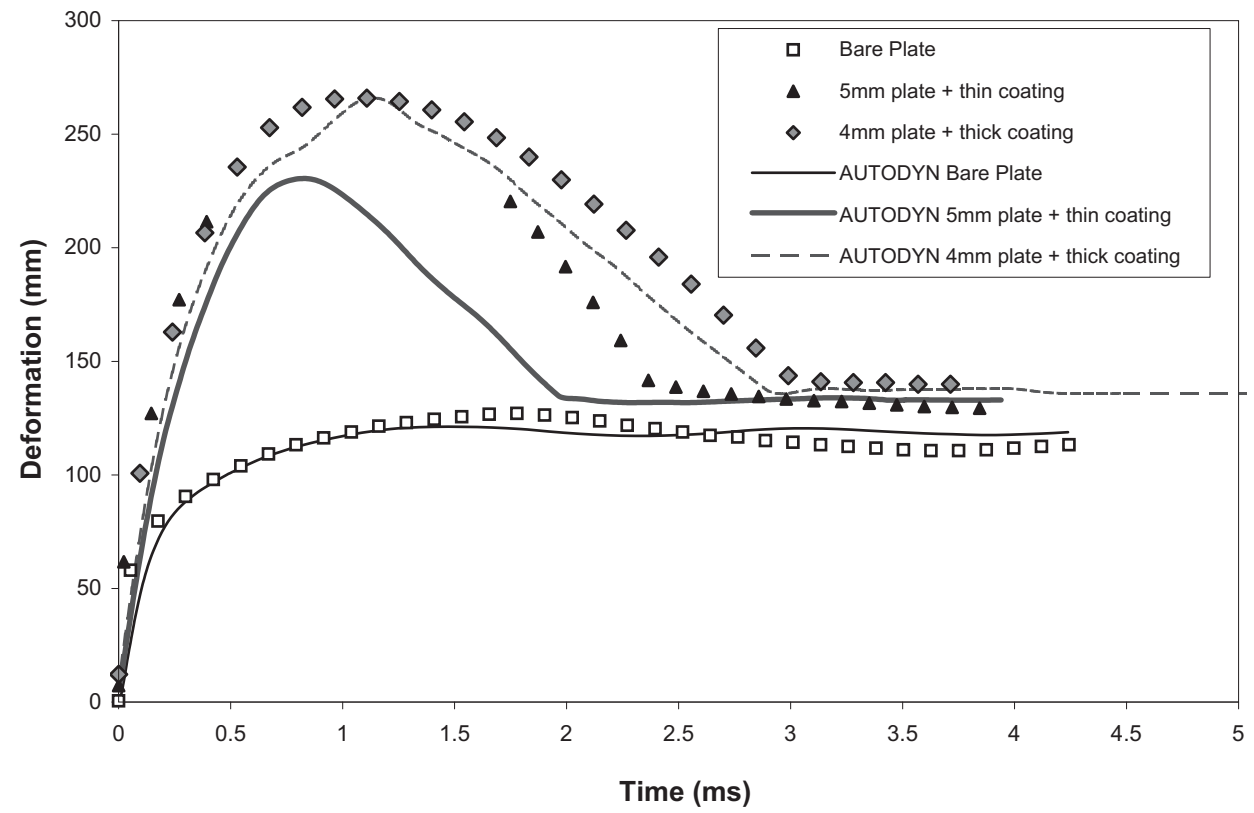

Fig. 15. Comparison of experimental and numerical results for the transient deformation of the plate centre using the updated Mooney-Rivlin constants. 
increasing deformation with increasing coating thickness, which was seen in the experiments. The simulation of the $7.7 \mathrm{~mm}$ coating over-predicted the plate deformation, whereas the simulation of the $15.7 \mathrm{~mm}$ coating under-predicted the plate deformation. Furthermore, the simulation with the $15.7 \mathrm{~mm}$ coating predicted a lower deformation than the $7.7 \mathrm{~mm}$ coating, which is the opposite of what was seen in the experiment. The discrepancy may be due to a number of factors, including the polyurea material model or the way in which the bond between the polyurea and the plate was modelled, and may be improved in future to achieve more consistent results.

An interesting observation seen in Fig. 16 was that in the numerical models, the polyureas which were not joined to the plates performed the best in terms of reducing residual plate deformation, compared to fully joined or de-bonding models. This could be due to the energy transfer from the blast to the polyurea through the steel. When the steel and polyurea are joined, the energy of the blast is transferred to the plate and polyurea as a system, and the energy is dissipated through the plastic deformation of the plate and polyurea. When the two are not joined, the energy transferred to the polyurea (which separates from the plate) reduces the energy transferred to the plate, compared to the previous scenario where the plate and polyurea are joined. However, the momentum of the polyurea in this case will cause it to become a hazard as it is accelerated through the air.

\section{Effect of coating thickness and location}

Further numerical investigations were conducted to assess the effect of coating thickness and coating location (front, back or sandwiched between two plates) on residual deformation. These models used the original Mooney-Rivlin constants as the final deformation was used as the performance measure. In each case the previous models were used with a constant yield stress of $400 \mathrm{MPa}$ for all of the plates. To speed up the simulations and simulate an ideal (unbreakable) bond, the polyurea coatings were joined to the plates such that no de-bonding would occur. The following configurations were simulated:

Coating thickness - The coating thickness was varied from $2 \mathrm{~mm}$ to $20 \mathrm{~mm}$. Elements of $2 \mathrm{~mm}$ thickness were used through the thickness of the polyurea. Steel plates of $4 \mathrm{~mm}$ and $5 \mathrm{~mm}$ thickness were modelled for the backing plates.

Front face coating - The effect of a front face coating was investigated for a $5 \mathrm{~mm}$ steel plate with a $7.7 \mathrm{~mm}$ polyurea coating by translating the plate from the front to the back of the polyurea, whilst keeping the gap between the charge and the impact face the same.

Sandwich configuration - A sandwich plate configuration was investigated using the $7.7 \mathrm{~mm}$ polyurea coating sandwiched between two $2.5 \mathrm{~mm}$ thick steel plates.

The responses of various thicknesses of bare plates were also simulated for comparison.

The results of the simulations are summarised in Fig. 17, which compares the final plate deformations based on their areal density. The black dots show the results for bare steel plates of different areal densities (i.e. different plate thicknesses). A curve has been applied to the data to improve clarity when comparing to the other data series. The curve for the bare plate shows that an increase in areal density results in a reduction in the final plate deformation. The increase in areal density has been achieved by increasing the plate thickness and hence plate mass and stiffness, whilst all other properties have been kept constant. The curve for the bare steel plate was used as a baseline for comparison with other

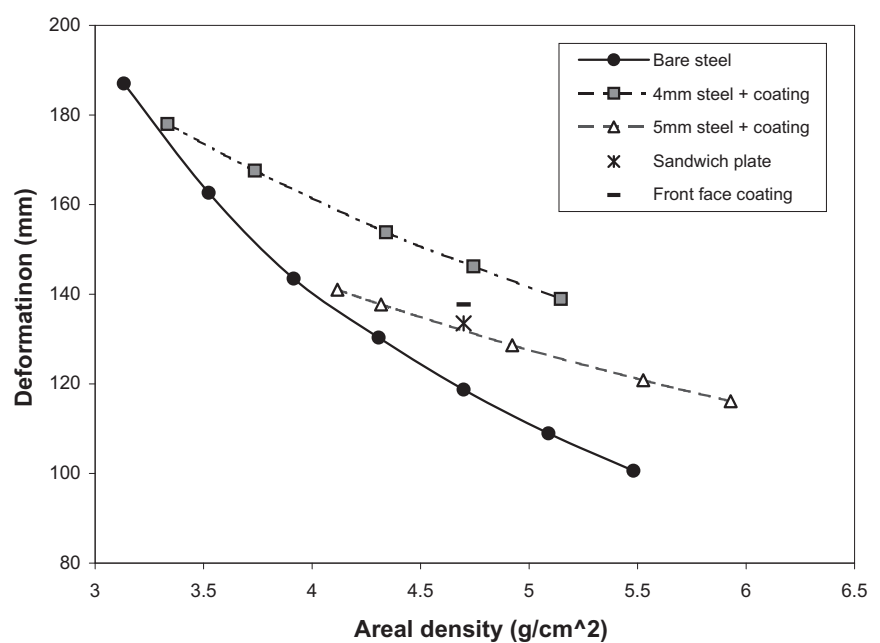

Fig. 17. Results from parametric modelling study, showing the effect of various coating and plate configurations of final plate deformation.

configurations which include polyurea coatings. For any given areal density within the range of the data set, any results which fall below the line for the bare plate have a lower final deformation, and hence better blast deformation protection, compared to the bare steel plate. In the present study, which considered various combinations of polyurea coatings and steel plates, no such improved results were found. The sandwich plate and front face coating configurations did not show any improvement over the bare steel plates. The $5 \mathrm{~mm}$ plate with the front face coating had a slightly higher deformation compared with the $5 \mathrm{~mm}$ plate with back face coating. This result agrees with results from Amini et al. $[5,6]$ who also found back face coatings more effective in mitigating blast.

These results show that for a given areal density a thick steel plate is more effective than a polyurea-coated thin steel plate in reducing deformation due to close in blast. This suggests that the plate deformation reduction achieved by adding a polyurea coating is primarily due to the fact that mass is being added to the system, with minimal change to the stiffness of the plate. The application of polyurea to an existing structure can be more practical than attaching additional steel, particularly if complex geometries are involved, however the potential for delamination must be considered. Polyureas may also be effective in preventing plate rupture $[5,23,6]$, which was not considered in this study.

\section{Summary and conclusions}

Experimental and numerical investigations were conducted to investigate whether the application of polyurea-coated plates would be advantageous to incorporate into blast resistant design compared to bare steel plates with the same areal density. The experimental studies showed that the bare steel plates dissipated the blast energy most effectively, resulting in the lowest plate deformations, compared to polyurea-coated plates of equivalent areal density. The plates with the polyurea coatings resulted in higher residual plate deformations, with the residual deformation increasing with coating thickness. High speed camera footage revealed de-bonding and hyperelastic extension of the polymer away from the back surface of the plate. This resulted in peak transient deformations for the polyurea coatings which were approximately double that of the bare steel plates. In a practical application, this could be hazardous to objects behind a blast resistant structure if a polyurea coating is applied to the back surface. 
Numerical modelling of steel plates with and without polyurea coatings was conducted using AUTODYN. The de-bonding of the polyurea was simulated using a thin layer of material with an applied failure stress. It was found that a failure stress of $80 \mathrm{MPa}$ best simulated the experimental results. Good agreement was found between the experimental and numerical results for the residual deformation of the plates; however the peak transient deformation was under-predicted for one of the plate configurations. Simulations also showed that in terms of residual deformation, steel plates performed better than polyurea-coated plates for any given areal density, regardless of the coating thickness or location.

\section{Acknowledgments}

The authors would like to thank Nigel St John, Russell Cairns and Ted Adamczyk for the preparation of the polyurea coatings. Many thanks also the DSTO trials team and assistance of the staff at P\&EE Graytown for their inputs into the conduct of the trial, and to the National Security task sponsors for funding the research. Thanks also to Roshdy Barsoum from the Office of Naval Research and Philip Dudt and Ken Nashon from the Naval Surface Warfare Centre, Carderock Division for their assistance with the polyurea material model, to Luke Mosse from Leap Australia for his help with AUTODYN and to Jinghan Lu and Sudharshan Raman from the University of Melbourne for their assistance with the high strain rate tensile testing.

\section{References}

[1] Davidson JS, Fisher JW, Hammons MI, Porter JR, Dinan RJ. Failure mechanisms of polymer-reinforced concrete masonry walls subjected to blast. J Struct Eng 2005;131:1194-205.

[2] Davidson JS, Porter JR, Dinan RJ, Hammons MI, Connell JD. Explosive testing of polymer retrofit masonry walls. J Perform Constr Facil 2004;18:100-6.

[3] Johnson CF, Slawson TR, Cummins TK, Davis JL. Concrete masonry unit walls retrofitted with elastomeric systems for blast loads. In: Parmentola JA, Rajendran AM, Bryzik W, Walker BJ, McCauley JW, Reifman J, Nasrabadi NM, editors. Transformational science and technology for the current and future force, Proceedings of the $24^{\text {th }}$ US Army Science Conference. Florida: World Scientific; 2005.

[4] Hoo Fat MS, Ouyang X, Dinan RJ. Blast response of walls retrofitted with elastomer coatings. Struct Mater 2004;15:129-38.
[5] Amini MR, Amirkhizi AV, Nemat-Nasser S. Numerical modeling of response of monolithic and bilayer plates to impulsive loads. Int J Impact Eng 2010;37: 90-102.

[6] Amini MR, Isaacs J, Nemat-Nasser S. Experimental investigation of response of monolithic and bilayer plates to impulsive loads. Int J Impact Eng 2010;37: 82-9.

[7] Matthews W. Services test spray-on vehicle armor. Vancouver, USA: Army Times; 2004.

[8] Ackland K, Anderson C, St John N. Polymeric coatings for enhanced protection of structures from the explosive effects of blast. In: Mendis P, Lai J, Dawson E, Abbass H, editors. Recent advances in security technology 2007, Proceedings of the 2007 RNSA Security Technology Conference. Melbourne: Australian Homeland Security Research Centre; 2007. p. 90-6.

[9] Bahei-El-Din YA, Dvorak GJ. Behavior of sandwich plates reinforced with polyurethane/polyurea interlayers under blast loads. J Sandw Struct Mater 2007;9:261-81.

[10] Gardner N, Wang E, Kumar P, Shulka A. Blast mitigation in a sandwich composite using graded core and polyurea interlayer. Exp Mech 2012;52: 119-33.

[11] Tekalur SA, Shulka A, Shivakumar K. Blast resistance of polyurea based layered composite materials. Comp Struct 2008;84:271-81.

[12] Grujicic M, Bell WC, Pandurangan B, He T. Blast-wave impact mitigation capability of polyurea when used as helmet suspension-pad material. Mater Des 2010;31:4050-65.

[13] Bluescope Steel. Bluescope steel 350 Xlerplate ${ }^{\circledR}$ steel datasheet. Port Kembla: Bluescope Steel Limited; 2009.

[14] Polyurethane specialty products group: VERSALINK ${ }^{\circledR}$ P-1000 OLIGOMERIC DIAMINE datasheet. Allentown, PA: Air Products and Chemicals Inc. Pub no. 140-05-050-US, <http://www.purmindex.net/purmpib/VersalinkP-1000. pdf $>$; 2005 [accessed 06.03.12]

[15] Isonate ${ }^{\circledR} 2143 \mathrm{~L}$; modified MDI. Midland, MI: The Dow Chemical Company, <http://dow.com/PublishedLiterature/dh_0035/0901b80380035efa.pdf > ; 2001 [accessed 01.05.12].

[16] Johnson GR, Cook WH. A constitutive model and data for metals subjected to large strains, high strain rates and high temperatures. In: Proceedings of the seventh international ballistics symposium, The Hague, 1983, p. $541-47$.

[17] Balden VH, Nurick GN. Numerical simulation of the post-failure motion of steel plates subjected to blast loading. Int J Impact Eng 2005;32:14-34.

[18] ANSYS. Release 11.0 documentation for ANSYS workbench. CA, USA: Century Dynamics Inc; 2008.

[19] Roland CM, Twigg JN, Vu Y, Mott PH. High strain rate mechanical behavior of polyurea. Polymer 2007;48:574-8.

[20] Amirkhizi AV, Isaacs J, Mcgee J, Nemat-Nasser S. An experimentally-based viscoelastic constitutive model for polyurea, including pressure and temperature effects. Philos Mag 2006;86:5847-66.

[21] Bahei-El-Din YA, Dvorak GJ, Fredricksen OJ. A blast-tolerant sandwich plate design with a polyurea interlayer. Int J Solid Struct 2006;43:7644-58.

[22] Yi J, Boyce MC, Lee GF, Balizer E. Large deformation rate-dependent stressstrain behavior of polyurea and polyurethanes. Polymer 2006;47:319-29.

[23] Barsoum RGS, Dudt PJ. The fascinating behaviors of ordinary materials under dynamic conditions, AMMTIAC Q vol. 4, no. 4 - countering the modern threat: armor for advanced protection. New York: Advanced Materials, Manufacturing, and Testing Information Analysis Center (AMMTIAC); 2010. 


\section{University Library}

\section{- M M I N E R VA A gateway to Melbourne's research publications}

Minerva Access is the Institutional Repository of The University of Melbourne

Author/s:

Ackland, Kathryn Patricia

Title:

Deformation of polyurea-coated steel plates under localised blast loading

Date:

2013

Citation:

Ackland, K. P. (2013). Deformation of polyurea-coated steel plates under localised blast loading. PhD thesis, Department of Infrastructure Engineering, The University of Melbourne.

Publication Status:

Unpublished

Persistent Link:

http://hdl.handle.net/11343/38232 